\title{
DIGITAL IMAGE PROCESSING APPLICATIONS IN THE IGNITION AND COMBUSTION OF CHAR/COAL PARTICLES
}

\author{
K.Annamalai \\ E.Kharbat \\ C.Goplakrishnan \\ Mechanical Engineering \\ Texas A\&M University \\ College Station, TX 77843
}

Topical Report

Prepared for

U.S.Department of Energy

Pittsburgh Energy Technology Center

Pittsburgh,Pa. 15236

Grant \# DE-FG22-90PC90310

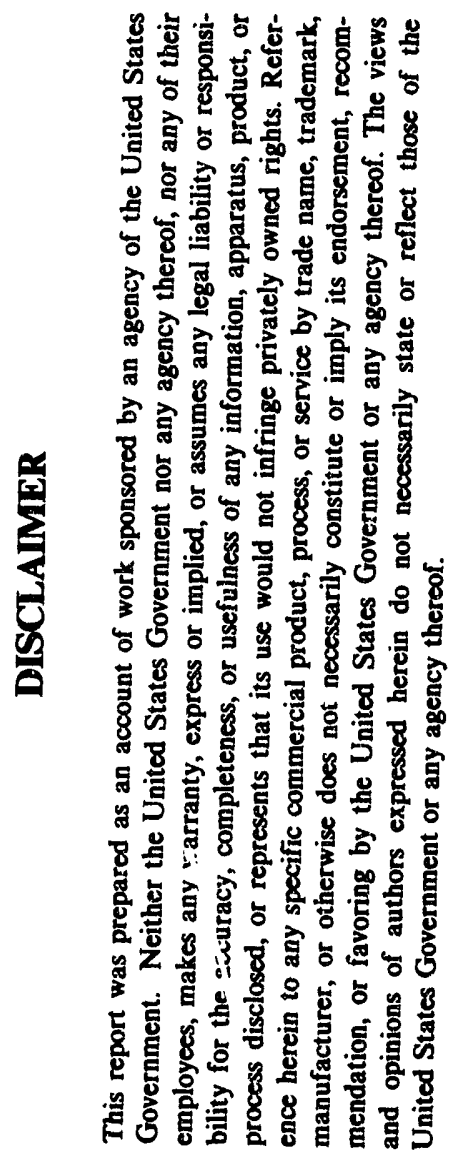

December 1992 


\section{DISCLAIMER}

A part of the present research was supported with a research grant from US nepartment of Energy/PETC Grant DE-FG22-90PC90310. However any opinions, findings, conclusions or recommendations do not: necessarily reflect the views of US Department of Energy. 


\section{DIGITAL IMAGE PROCESSING APPLICATIONS IN THE IGNITION AND COMBUSTION OF CHAR/COAL PARTICLES}

\section{ABSTRACT}

The importance of ignition and combustion of coal, improved flame stability and combustion efficiency, has fueled the minds of many investigators to conduct fundamental experiments in this field. Based on the premise that the ignitability of a spray suspension must depend on the ignition characteristics of isolated particles, most of these studies have been concentrated on the ignition and combustion of isolated coal particles. While isolated particle studies may be useful for dilute coal suspensions, its application to practical burners is limited, since ignition usually occurs in a dense stream region. Such a dense mode of combustion in the oxygen deficient zones leads to increased combustion time, reduced flame stability, incomplete combustion and increased emission of $\mathrm{HC}, \mathrm{CO}$ and other pollutants. Thus it is imperative to determine the ignition characteristics of a large number of interacting particles.

Recently, investigators have employed new techniques and advanced computer technology to investigate the behavior of char/coal under combustion. Such techniques provided new concepts and insights in combustion science. A recent technique, digital image processing, is employed in this research study in order to visually investigate the ignition and combustion characteristics of isolated char/coal particles as well as the effect of interactive combustion in two-particle char/coal arrays. Preliminary 
experiments are conducted on miniature isolated candles as well as two-candle arrays. Results indicate that as the candle staris to burn, a flame is formed around its wick including a black inner zone possibly due to the formation of fuel rich zone. With increased inter-candle spacing for the two-candle ariays, the burn time first decreases and later increases. Ignition of the high volatile isolated coal particles in vitiated environment seems to occur heterogeneously at the leading edge of the particle. Volatiles which are ejected upward as jets in the direction of the convective flow, burn in the gas phase homogeneously and form a wake flame; a black inner zone (unburned volatile) is formed. Also, intermittent volatile ignition and combustion are observed to occur during the combustion process, for a few of the experiments. The medium volatile coal particles ignite faster than the high volatile coal; but the intermittent ignition is not observed. The low volatile isolated coal particles combust in shorter time. The isolated char particles ignite at the surface of the particle heterogeneously with little volatile ejected, yet are not sufficient to form a volatile flame, resulting in a subsequent heterogeneous combustion. A group flame is formed for the two-particle arrays at closer interparticle spacing. Also, intermittent ignition does not occur for the high volatile particles when the two particles are at farther distances which suggests that radiation interaction between the particles might be occurring. The char arrays experience heterogeneous ignition at the leading edge; combustion proceeds heterogeneously. 


\section{TABLE OF CONTENTS}

CHAPTER

Page

I INTRODUCTION $\ldots \ldots \ldots \ldots \ldots \ldots \ldots \ldots \ldots \ldots$

I.1 Scope of the Present Work $\ldots \ldots \ldots \ldots \ldots \ldots \ldots$

II LITERATURE REVIEW $\ldots \ldots \ldots \ldots \ldots \ldots \ldots$

II.1 Ignition of Isolated Char/Coal Particles $\ldots \ldots \ldots \ldots \ldots \ldots 8$

II.2 Combustion of Isolated Char/Coal Particles . . . . . . . 21

II.3 Interactive Combustion of Char/Coal Particles . . . . . . 24

II.4 Image Processing Applications in Combustion . . . . . . . 28

III OBJECTIVES $\ldots \ldots \ldots \ldots \ldots \ldots \ldots \ldots \ldots \ldots \ldots \ldots \ldots$

IV OVERVIEW OF DIGITAL IMAGE PROCESSING $\ldots \ldots \ldots \ldots 34$

IV.1 Components of a Digital Image Processing System . . . . . 36

IV.2 Lenses and Light Values . . . . . . . . . . . . . 42

V EXPERIMENTAL DESCRIPTION $\ldots \ldots \ldots \ldots \ldots \ldots$

V.1 Experimental Setup $\ldots \ldots \ldots \ldots \ldots \ldots \ldots \ldots \ldots$

V.2 Experimental Procedure ...............68 68

VI RESULTS AND DISCUSSION $\ldots \ldots \ldots \ldots \ldots \ldots \ldots \ldots$

VI.1 Isolated Candles $\ldots \ldots \ldots \ldots \ldots \ldots \ldots \ldots \ldots \ldots 71$

VI.2 Two-Candle Arrays $\ldots \ldots \ldots \ldots \ldots \ldots \ldots \ldots \ldots$

VI.3 Isolated Coal Particles . . . . . . . . . . . . . 82

VI.4 Isolated Char Particles . . . . . . . . . . . . . . . . . . 113

VI.5 Coal Two-Particle Arrays . . . . . . . . . . . . . . . . 122

VI.6 Char Two-Particle Arrays . . . . . . . . . . . . . . . . 165

VII CONCLUSIONS $\ldots \ldots \ldots \ldots \ldots \ldots \ldots \ldots \ldots \ldots \ldots$

VIII RECOMMENDATIONS . . . . . . . . . . . . . . . . . . . 184 
Page

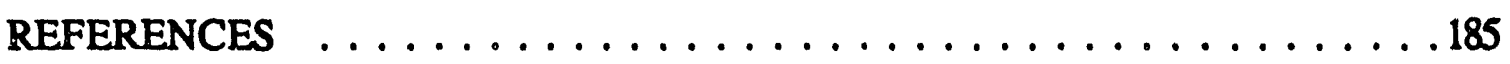

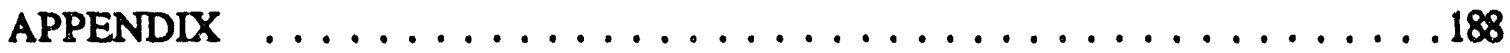

A LENS SELECTION CRITERIA $\ldots \ldots \ldots \ldots \ldots \ldots \ldots \ldots 188$

B EXPERIMENTAL DATA $\ldots \ldots \ldots \ldots \ldots \ldots \ldots \ldots$ 


\section{LIST OF TABLES}

Table

Page

1 Isolated Candle Mass Variation with Time . . . . . . . . . . . . . 72

2 Physical and Chemical Analysis of Coal ................ 83

3 Base Case and Parametric Conditions for Isolated Char/Coal Particles . . . . . . . . . . . . . . . . . . . . . . . . 84

4 Flame Characteristics of High Volatile Isolated Coal Particle . . . . . . . . . . . . . . . . . . . . . . . 93

5 Flame Characteristics of Medium Volatile Isolated Coal Particle ................................ 108

6 Flame Characteristics of Low Volatile Isolated Coal Particle ............................... 115

7 Base Case and Parametric Conditions for Char/Coal Two-Particle Arrays . . . . . . . . . . . . . . . . . . . . 123

8 Combustion Characteristics of Large High Volatile Coal Two-Particle Arrays . . . . . . . . . . . . . . . . . . . . 161

9 Combustion Characteristics of Intermediate High Volatile Coal Two-Particle Arrays . . . . . . . . . . . . . . . . . . . . . 161

10 Combustion Characteristics of Small High Volatile Coal Two-Particle Arrays . . . . . . . . . . . . . . . . . . . . . . 162

11 Combustion Characteristics of Medium Volatile Coal Two-Particle Arrays . . . . . . . . . . . . . . . . . . . . . . 180

12 Variation of Mass with Time for Two-Candle Array $(l=6 \mathrm{~mm}) \ldots$

13 Variation of Mass with Time for Two-Candle Array $(l=9 \mathrm{~mm}) \ldots \ldots 3$ 
14 Variation of Mass with Time for Two-Candle Array $(l=12 \mathrm{~mm}) \ldots 194$

15 Variation of Mass with Time for Two-Candle Array $(l=15 \mathrm{~mm}) \ldots$

16 Variation of Mass with Time for Two-Candle Array $(l=17 \mathrm{~mm}) \ldots$

17 Variation of $40 \%$ Burn Time with Inter-Candle Spacing for Two Candle Arrays . . . . . . . . . . . . . . . . . . . . . 197 


\section{LIST OF FIGURES}

Figure

1 Net Generation of Electricity by Energy Source

Page [Annual Energy Review, 1991] . . . . . . . . . . . . . . 2

2 Illustration of Interactive Combustion $\ldots \ldots \ldots \ldots \ldots \ldots \ldots$

3 Difference Between Oil and Coal Combustion ..........6 6

4 Single and Double Film Models for Char/Coal Combustion . . . . . . 13

5 Ignition Temperature Variation of Coal and Anthracite Particles . . . . 16

6 Variation of Ignition Temperature for Gas (Homogeneous) Ignition and Carbon (Heterogeneous) Ignition with Particle Size . . . . . . . 19

7 Ignition at Zero Gravity for a Binary Coal Particle Array $\ldots \ldots \ldots 26$

8 Ignition at a Normal Gravity for a Binary Coal Particle Array . . . . . 27

9 Flame Propagation Velocity vs Interparticle Separation Distance . . . . . 29

10 Illustration of a Digital Grid (Bitmap) $\ldots \ldots \ldots \ldots \ldots \ldots \ldots$

11 A Hierarchy of Digital Image Processing Operations $\ldots \ldots \ldots \ldots . \ldots 41$

12 Schematic of Flat Flame Bumer . . . . . . . . . . . 46

13 Schematic of Flat Flame Bumer Facility . . . . . . . . . 48

14 Image Processing Block Diagram $\ldots \ldots \ldots \ldots \ldots \ldots \ldots \ldots$

15 Schematic of the CID2250D Camera $\ldots \ldots \ldots \ldots \ldots \ldots$

16 Architecture of Image Processing Board . . . . . . . . . . 57

17 Variation of Mass with Time for Isolated Candle $\ldots \ldots \ldots \ldots$. . . . 74 
18a The Burning Sequence for Isolated Candle (Numbers are in msec) . . . . 75

18b Images of the Burning Sequence with Boundary Detection Function for Isolated Candle $\ldots \ldots \ldots \ldots \ldots \ldots$

19 Variation of Normalized Candles Mass with Time for Candle Arrays . . . . . . . . . . . . . . 78

20 Variation of $40 \%$ Burn Time with Inter-Candle Spacing for Candle Arrays $\ldots \ldots \ldots \ldots \ldots \ldots \ldots \ldots \ldots \ldots$

21a The Burning Sequence for Two-Candle Array at $l / a=6 \mathrm{~mm} \ldots \ldots . .80$

21b Images of the Burning Sequence for Two-Candle Array with Boundary Detection Function $\ldots \ldots \ldots \ldots \ldots \ldots$

22 A Typical Sketch of Combustion of Isolated Coal Particle in Vitiated Environment $\ldots \ldots \ldots \ldots \ldots \ldots \ldots \ldots$

23a Ignition and Combustion Sequence for High Volatile Isolated Coal Particle (Numbers are in msec) . . . . . . . . . . 86

23b Images of Ignition and Combustion Sequence with Boundary Detection Function for High Volatile Isolated Coal Particle . . . . . . 88

24 A Typical Flame Geometry of Isolated High Volatile Coal Particle . . . 92

25 Flame Characteristics of Isolated High Volatile Coal Particle . . . . . . 95

26 Variation of Flame Tip Velocity with Time for Isolated High Volatile Coal Particle . . . . . . . . . . . . . . . . . 97

27a Intermittent Ignition and Combustion Sequence for High Volatile Coal Particle . . . . . . . . . . . . . . . . 98

27b Images of Intermittent Ignition and Combustion Sequence with Boundary Detection Function . . . . . . . . . . . . . 99 
28a Ignition and Combustion Sequence for Isolated Medium

Vulatile Coal Particle . . . . . . . . . . . . . . . . . . . . . . . . . . . 101

28b Images of Ignition and Combustion Sequence with Boundary

Detection Function for Isolated Medium Volatile Coal Particle . . . . . . 103

29 Flame Characteristics of Isolated Medium Volatile Coal Particle . . . . . 105

30 Flame Tip Velocity of Isolated Medium Volatile Coal Particle . . . . . 106

31a Ignition and Combustion Sequence for Isolated Low

Volatile Coal Particle . . . . . . . . . . . . . . . . . . . . . . . . 110

31b Images of Ignition and Combustion Sequence with Boundary

Detection Function for Isolated Low Volatile Coal Particle . . . . . . . 111

32 Flame Characteristics of Isolated Low Volatile Coal Particle . . . . . . 112

33 Flame Tip Velocity of Isolated Low Volatile Coal Particle . . . . . . . . 114

34 Comparison of Flame Height for Isolated Coal Particles . . . . . . . . . 116

35 Comparison of Flame Width for Isolated Coal Particles . . . . . . . . 117

36 Comparison of Flame Tip Velocity for Isolated Coal Particles . . . . . 118

37 Comparison of Ignition, Buming, and Combustion Times for Isolated Coal Particles . . . . . . . . . . . . . . . . . . . . . 119

38a Ignition and Combustion Sequence for Isolated Char Particle . . . . . . 120

38b Images of Ignition and Combustion Sequence With Boundary Detection Function for Isolated Char Particle . . . . . . . . . . . . . . 121

39 A Typical Sketch of Combustion of Two-Particle Array in Vitiated Environment ... . . . . . . . . . . . . . . . . . . . 124

40 Variation of Flames Merging Time with Interparticle Spacing for Large High Volatile Coal Two-Particle Array . . . . . . . . . . . . . 126 
41 Variation of Flames Merging Time with Interparticle Spacing for Intermediate High Volatile Coal Two-Particle Array . . . . . . . . . 127

42 Variation of Flames Merging Time with Interparticle Spacing for Small High Volatile Coal Two-Particle Array . . . . . . . . . . . 128

43a Ignition and Combustion Sequence for Large High Volatile Coal Two-Particle Array $(l=2.2 \mathrm{~mm})$, Numbers are in msec . . . . . 129

43b Images of Ignition and Combustion Sequence With Boundary Detection Function $(l=2.2 \mathrm{~mm}) \ldots \ldots . \ldots 130$

44a Ignition and Combustion Sequence for Large High Volatile Coal Two-Particle Array $(l=4.86 \mathrm{~mm}) \ldots \ldots . \ldots 131$

44b Images of Ignition and Combustion Sequence With Boundary Detection Function $(l=4.86 \mathrm{~mm}) \ldots \ldots . . . \ldots . . . . .132$

45a Ignition and Combustion Sequence for Large High Volatile Coal Two-Particle Array $(l=7.26 \mathrm{~mm}) \ldots \ldots . \ldots 133$

45b Images of Ignition and Combustion Sequence With Boundary Detection Function $(l=7.26 \mathrm{~mm}) \ldots \ldots \ldots$

46a Ignition and Combustion Sequence for Large High Volatile

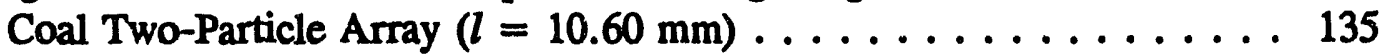

$46 \mathrm{~b}$ Images of Ignition and Combustion Sequence With Boundary Detection Function $(l=10.60 \mathrm{~mm}) \ldots \ldots . . \ldots . . . . . . .136$

47a Ignition and Combustion Sequence for Large High Volatile Coal Two-Particle Array $(l=13.45 \mathrm{~mm}) \ldots \ldots . \ldots 137$

47b Images of Ignition and Combustion Sequence With Boundary Detection Function $(l=13.45 \mathrm{~mm}) \ldots \ldots . . \ldots 138$

48a Ignition and Combustion Sequence for Large High Volatile Coal Two-Particle Array $(l=15.88 \mathrm{~mm}) \ldots \ldots . . . . . . . . . .139$ 
Figure

Page

48b Images of Ignition and Combustion Sequence With

Boundary Detection Function $(l=15.88 \mathrm{~mm}) \ldots \ldots$. . . . . . . . 140

49a Ignition and Combustion Sequence for Intermediate High Volatile

Coal Two-Particle Array $(l=2.13 \mathrm{~mm}) \ldots \ldots \ldots 141$

49b Images of Ignition and Combustion Sequence With

Boundary Detection Function $(l=2.13 \mathrm{~mm}) \ldots \ldots . . . \ldots . . . . .142$

50a Ignition and Combustion Sequence for Intermediate High Volatile

Coal Two-Particle Array $(l=4.53 \mathrm{~mm}) \ldots \ldots 143$

$50 \mathrm{~b}$ Images of Ignition and Combustion Sequence With

Boundary Detection Function $(l=4.53 \mathrm{~mm}) \ldots \ldots . . . \ldots . . . . .144$

51a Ignition and Combustion Sequence for Intermediate High Volatile

Coal Two-Particle Array $(l=7.12 \mathrm{~mm}) \ldots \ldots 145$

51b Images of Ignition and Combustion Sequence With

Boundary Detection Function $(l=7.12 \mathrm{~mm}) \ldots \ldots \ldots \ldots$

52a Ignition and Combustion Sequence for Intermediate High Volatile

Coal Two-Particle Array $(l=10.34 \mathrm{~mm}) \ldots \ldots \ldots$

52b Images of Ignition and Combustion Sequence With

Boundary Detection Function $(l=10.34 \mathrm{~mm}) \ldots \ldots . . . \ldots . . . . .148$

53a Ignition and Combustion Sequence for Intermediate High Volatile

Coal Two-Particle Array $(l=13.42 \mathrm{~mm}) \ldots \ldots$. . . . . . . . . . . 149

53b Images of Ignition and Combustion Sequence with

Boundary Detection Function $(l=13.42 \mathrm{~mm}) \ldots \ldots . . . \ldots . . . . .150$

54a Ignition and Combustion Sequence for Small High Volatile

Coal Two-Particle Array $(l=2.05 \mathrm{~mm}) \ldots \ldots . \ldots 151$

54b Images of Ignition and Combustion Sequence with

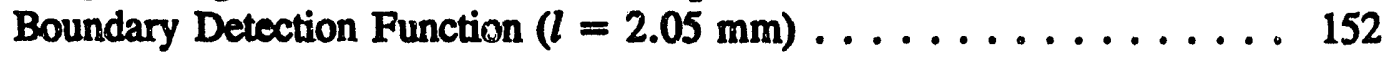


Figure

Page

55a Ignition and Combustion Sequence for Small High Volatile Coal Two-Particle Array $(l=4.67 \mathrm{~mm}) \ldots \ldots \ldots \ldots \ldots \ldots$

55b Images of Ignition and Combustion Sequence with Boundary Detection Function $(l=4.67 \mathrm{~mm}) \ldots \ldots \ldots \ldots . \ldots 154$

56a Ignition and Combustion Sequence for Small High Volatile Coal Two-Particle Array $(l=7.1 \mathrm{~mm}) \ldots \ldots \ldots \ldots \ldots \ldots$

56b Images of Ignition and Conibustion Sequence with Boundary Detection Function $(l=7.1 \mathrm{~mm}) \ldots \ldots \ldots \ldots$

57a Ignition and Combustion Sequence for Small High Volatile Coal Two-Particle Array $(l=10.16 \mathrm{~mm}) \ldots \ldots \ldots \ldots \ldots \ldots . \ldots 157$

57b Images of Ignition and Combustion Sequence with Boundary Detection Function $(l=10.16 \mathrm{~mm}) \ldots \ldots \ldots \ldots \ldots . \ldots 158$

58 Variation of Flames Merging Time with Interparticle Spacing for High volatile Coal Two-Particle Arrays . . . . . . . . . . . 159

59a Ignition and Combustion Sequences for High Vola: ile Two-Particle Array with Vertical Geometry $\ldots \ldots \ldots \ldots \ldots \ldots$. 53

59b Images of Ignition and Combustion Sequence With Boundary Detection Function (Vertical Geometry) . . . . . . . . . . 164

60a Ignition and Combustion Sequence for Medium Volatile Coal Two-Particle Array $(l=2.54 \mathrm{~mm})$, Numbers are in $\mathrm{msec} \ldots \ldots 166$

$60 \mathrm{~b}$ Images of Ignition and Combustion Sequence with Boundary Detection Function $(l=2.54 \mathrm{~mm}) \ldots \ldots \ldots \ldots$

61a Ignition and Combustion Sequence for Medium Volatile Coal Two-Particle Array $(l=4.67 \mathrm{~mm}) \ldots \ldots \ldots \ldots \ldots . \ldots 168$

61b Images of Ignition and Combustion Sequence with Boundary Detection Function $(l=4.67)$ 
62a Ignition and Combustion Sequence for Medium Volatile

Coal Two-Particle Array $(l=7.85 \mathrm{~mm}) \ldots \ldots \ldots \ldots \ldots$

62b Images of Ignition and Combustion Sequence with

Boundary Detection Function $(l=7.85 \mathrm{~mm}) \ldots \ldots \ldots \ldots \ldots 17$

63a Ignition and Combustion Sequence for Medium Volatile

Coal Two-Particle Array $(l=13.58 \mathrm{~mm}) \ldots \ldots \ldots \ldots \ldots \ldots 172$

63b Images of Ignition and Combustion Sequence with

Boundary Detection Function $(l=13.58 \mathrm{~mm}) \ldots \ldots \ldots \ldots$

64a Ignition and Combustion Sequence for Medium Volatile

Coal Two-Particle Array $(l=15.87 \mathrm{~mm}) \ldots \ldots \ldots \ldots \ldots \ldots . \ldots 174$

64b Images of Ignition and Combustion Sequence with

Boundary Detection Function $(l=15.87 \mathrm{~mm}) \ldots \ldots \ldots \ldots \ldots$

65a Ignition and Combustion Sequence for Medium Volatile

Coal Two-Particle Array $(l=17.98 \mathrm{~mm}) \ldots \ldots \ldots \ldots \ldots \ldots$

65b Images of Ignition and Combustion Sequence with

Boundary Detection Function $(l=17.98 \mathrm{~mm}) \ldots \ldots \ldots \ldots . \ldots 177$

66 Variation of Flames Merging Time with Interparticle

Spacing for Medium volatile Coal Two-Particle Arrays . . . . . . 178

67 Comparison of Flames Merging Time variation with Interparticle

Spacing for High and Medium Volatile Coal Two-Particle Arrays . . . 179

68a Ignition and Combustion Sequences for Char

Two-Particle Array $(l=2.20 \mathrm{~mm}) \ldots \ldots \ldots \ldots \ldots \ldots \ldots$

68b Images of Ignition and Combustion Sequence with Boundary

Detection Function for Char Two-Particle Array . . . . . . . . . . 182

69 Variation of Focal Length with Magnification . . . . . . . . . . 190

70 Variation of Mass (Regression) with Time for Candles . . . . . . . . 198 


\section{CHAPTER I}

\section{INTRODUCTION}

Electric power generation in North America comes from various sources [Annual Energy Review, 1991] such as nuclear power, hydroelectric energy, conventional and breeder reactors, and fossil fuels (Figure 1). While the major portion of fossil fuel is consumed for electric power generation, the rest is used for transportation such as cars, ships and aircrafts, and for commercial and residential purposes. Fossil fuels consist mainly of coal, oil (petroleum), and natural gas. The largest petroleum consumer is transportation. Transportation reliance on oil, air pollution from transportation sources, oil's fluctuating prices, limited supply and renewability, and the increased dependency on foreign sources, are all influential factors, that increased research studies on alternative fuels. An alternative which exists abundantly in the United States is coal. The worldwide consumption of coal is expected to increase in the upcoming decades.

While coal is an attractive alternative fuel, obstacles such as increased pollution and the conversion of oil fueled burners to coal fired burners need to be overcome. Thus, extensive experimental studies are essential to identify the viable solutions for these obstacles.

Most of the industrial oil spray burners, jet engines and coal fired utility boilers 
By Major Energy Source, 1001

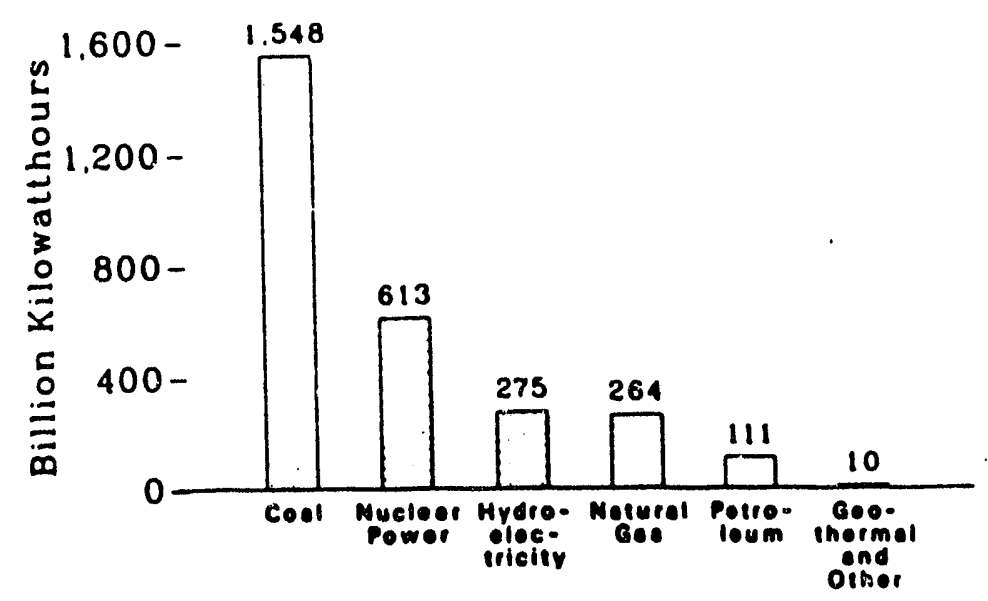

Total, 1948-1891

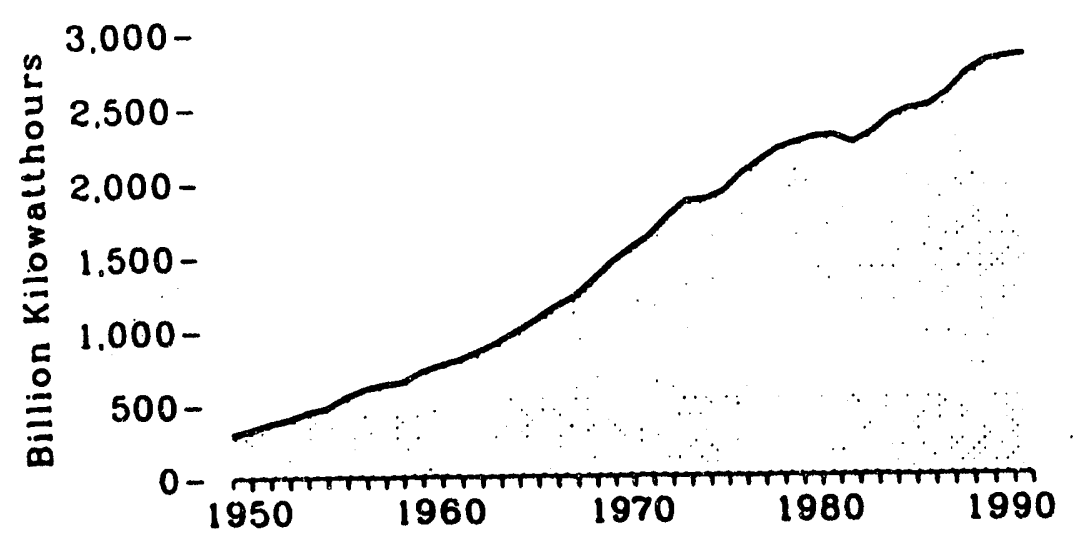

Figure 1. Net Generation of Electricity by Energy Source [Annual Energy Review, 1991] 
use very dense clouds of drops or particles suspended in gas, since atomization of liquid and pulverization of solid provide high ratio of surface area to volume of fuel injected. This provides high intensity combustion and good flame stability behavior at widely varying loads. It is thus of utmost importance to understand the ignition and combustion processes of coal and oil sprays. While the previous studies conducted in this field assumed the ISOlated Particle Combustion (ISOC) model, for the interpretation of experimental data and for analytical modeling, fuel sprays consist of a large number of interacting drops or particles. In recent decades, the interactive combustion modeling has been introduced.

In the field of coal combustion, results are analogous to those obtained from liquid fuel combustion. This is due to the fact that basic combustion theories apply to both processes with some differences to compensate for the difference in the ignition and combustion characteristics of a liquid drop and a solid particle. An isolated droplet burning in air has its own envelope flame (Figure 2a). If another burning drop is brought near the droplet, then the flames at first deform due to a depletion of oxygen between the drops. Once the drops are close to each other, the flames merge and form a common flame around both drops (Figure $2 b$ ). This is the simplest definition of group combustion. Similarly, if a large number of drops are brought close together, then a common flame is formed around all of the drops (Figure 2c). A similar behavior is expected for coal particles, since coal contains typically about $40-50 \%$ volatile matter (VM); the volatile may establish a group flame under dense conditions. 
a.)

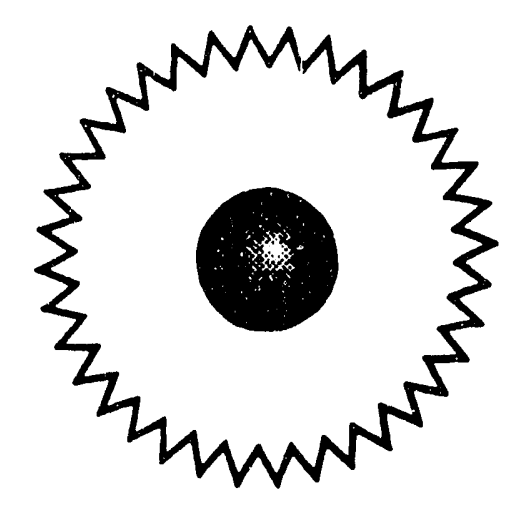

\section{Isolated Drop}

b.)

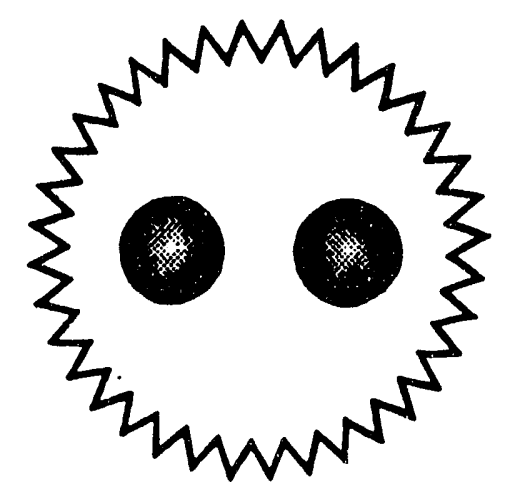

Two Drops

c.)

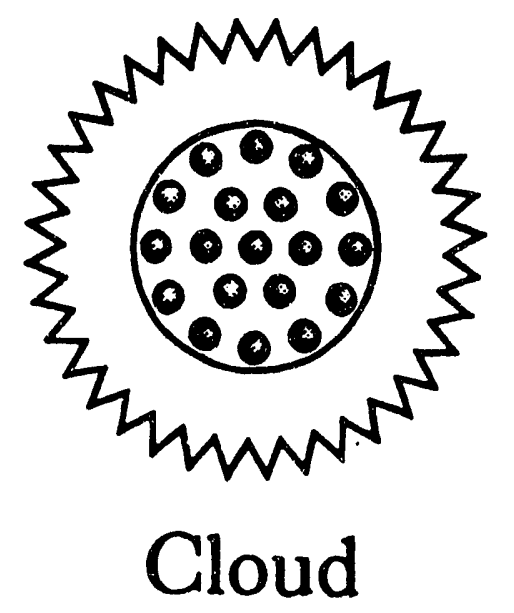

Figure 2. Illustration of Interactive Combustion 
However, it is important at this point to realize the differences in the processes involved in the combustion of oil drops and the combustion of coal particles. While droplet evaporation is a purely physical process (Figure 3a), coal particles release combustible gases (volatile) through a chemical decomposition process at high temperatures (pyrolysis). In addition, the morphology and composition of coals vary widely from one coal type to the other. Once the coal particles have completely pyrolyzed, the carbon in coal, fixed carbon (FC), can react heterogeneously with $\mathrm{O}_{2}$ to form $\mathrm{CO}$ and $\mathrm{CO}_{2}$. Thus, ignition of coal particles can occur via two routes; (i.) homogeneous ignition, where released volatile ignite in the gas phase (Figure 3b) or (ii.) heterogeneous ignition, where carbon oxidizes at the particle surface (Figure 3c). Once ignited, an envelope of volatile flame may be formed around the isolated particle. The ratio of flame to particle radii was observed to be of the order of 10 to 12 [Juniper and Wall, 1980].

In the present work, new techniques of digital image processing are employed in order to explore the ignition and combustion characteristics of isolated char/coal particles as well as to determine the interactive combustion effects in two-particle char/coal arrays.

\section{I.1 Scope of the Present Work}

While image processing techniques have been employed in almost all aspects of scientific research studies, its applications in the area of combustion of char/coal particles 
a.)

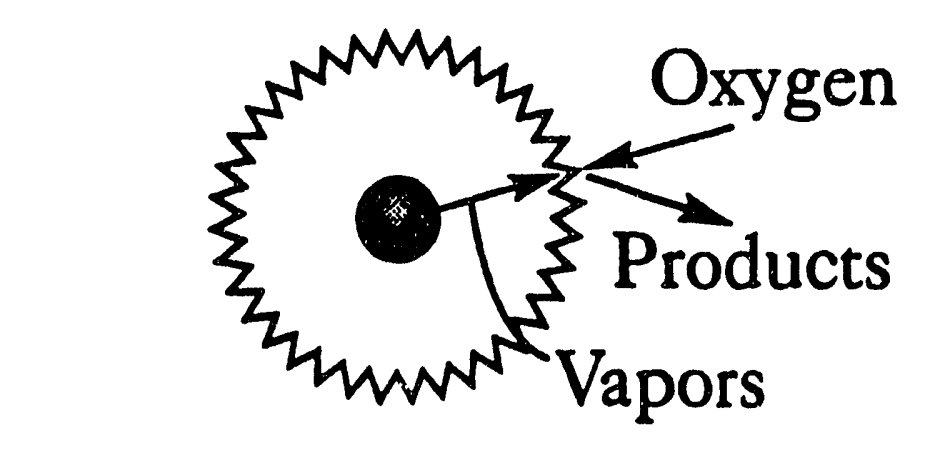

Oil Drop Combustion

b.)

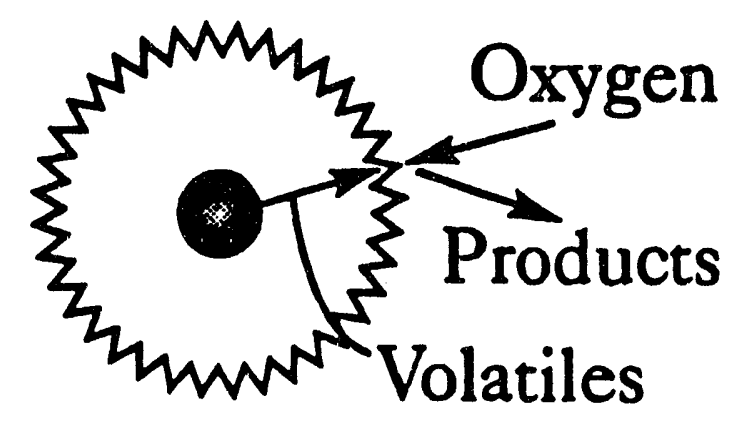

Homogeneous Coal Particle Combustion

c.)

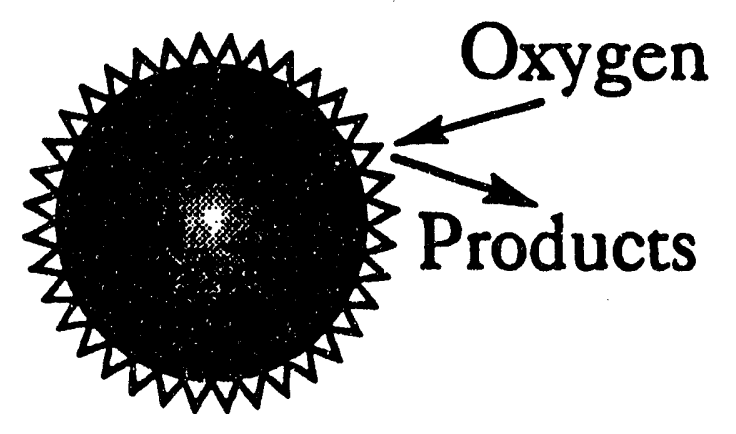

Heterogeneous Coal Particle Combustion

Figure 3. Difference between Oil and Coal Combustion 
are very limited. The aim of the present work is to develop a digital image processing system and to employ it in studying the ignition and combustion of char/coal particles. Preliminary experiments will be first performed on miniature candles in order to illustrate the effect of the group/interactive combustion on the their burning rate. Subsequently studies will be conducted on isolated and two-particle char/coal arrays. The ignition and combustion characteristics include ignition and burning times, flame location and shape, and the effect of interparticle spacing on the combustion of twoparticle arrays. A basic understanding of the char/coal isolated particles and two-particle arrays combustion characteristics is expected to improve our knowledge of practical coal combustors, involving dense streams of particles. 


\section{CHAPTER II}

\section{LITERATURE REVIEW}

This chapter presents a review of literature dealing with ignition and combustion of isolated char/coal particles followed by a review of the literature dealing with the interactive combustion of char/coal particles. Since digital image processing techniques were employed in this research study, a review of some of the previous work employing these techniques in combustion is also included.

\section{II.1 Ignition of Isolated Char/Coal Particles}

Prior to reviewing ignition and combustion processes mentioned above, elementary concepts and definitions of char/coal particle ignition and combustion are presented here.

\section{II.1.1 What is Coal?}

The term "Coal" describes a substance that is extremely varied. Coal is a complex polymer consisting of elements $\mathrm{C}, \mathrm{H}, \mathrm{N}$, and $\mathrm{O}$. It contains combustibles, moisture, inorganic mineral matter originating from dissolved salts in water and extrinsic ash. The inorganic matter can include a number of minerals, such as pyrite, quartz, calcite, clays-almost any naturally occurring element can be found in coals in small to trace amounts. Coal is usually thought of as hard, shiny, and black; but some coals can be soft that they almost crumble, and many are brown in color. These differences are 
caused by the differences in the composition of the coal. A useiul way employed to classify coal is to perform a "proximate analysis". This analysis provides information about four important characteristics of the coal: (i.) the amount of moisture in the coal, which influences its behavior; (ii.) the amount of volatile matter (water, gases, oil, and tar) that is released when the coal is heated and starts to decompose chemically into its constituents; (iii.) the fixed carbon content (the amount of carbon material after volatile matter has been released); and (iv.) the ash content, which is determined by the inorganic mineral matter present in the coal initially. As part of this analysis, the heating value-the amount of energy released when coal is burned-is also determined. These determinations then allow the coal to be classified.

Upon heating in an inert atmosphere, volatile matter (VM) is evolved due to thermal decomposition of the solid normally referred to as pyrolysis. Volatile matter is defined as the fraction of solid fuels which can be released as combustible gases. Since coal is a compact aged form of biomass (plant debris, 70-80\% VM), it consists of 10$50 \%$ VM depending upon the age of coal. Volatile matter decreases as the period of aging increases. This is due to the gradual release of the hydrocarbon gases (dehydrogenation). Coal is ranked as low, medium and high according to its content of VM. As volatile matter decreases, coal rank increases. Typically, a medium rank coal consists of $40 \%$ volatile matter with the volatile species ranging from $\mathrm{CH}_{4}, \mathrm{CO}, \mathrm{Co}_{2}$, $\mathrm{H}_{2} \mathrm{O}$ etc. to tar, and $60 \%$ fixed carbon (FC). The densities of coal/char range from 1100 $\mathrm{kg} / \mathrm{m}^{3}$ for low rank coals to $2330 \mathrm{~kg} / \mathrm{m}^{3}$ for high density pyrolytic graphite. 
In boilers, the typical combustion time (order of $1 \mathrm{~s}$ ) is dominated by the time required for the heterogeneous combustion of the residual char particle, while the pyrolysis time is on the order of $1 / 10$ to $1 / 100$ th of the total burning time. Coal of high reactivity normally has a smaller ignition time ( by a factor of 10) compared to coal of low reactivity. Chars of low rank are normally more reactive than chars of high rank due to increased porosity.

\section{II.1.2 Coal Pyrolysis}

Pyrolysis is referred to the thermal decomposition of a coal particle when placed in a hot furnace. Pyrolysis is somewhat similar to vaporization, however, it is a relatively slow chemical process compared to rapid physical vaporization of oil drops. As a coal particle pyrolyzes, the particle density decreases as a result of increased porosity. The more volatile released, the greater the internal surface area available for heterogeneous reactions. Typically, bituminous coal pyrolyzes at about $700 \mathrm{~K}$ as in the case of most plastics. Pyrolysis products range from lighter volatile: $\mathrm{CH}_{4}, \mathrm{C}_{2} \mathrm{H}_{4}, \mathrm{C}_{2} \mathrm{H}_{6}$, $\mathrm{CO}, \mathrm{CO}_{2}, \mathrm{H}_{2}, \mathrm{H}_{2} \mathrm{O}$ etc. to heavier. Invariably tars crack to form $\mathrm{CH}_{4}, \mathrm{C}_{2} \mathrm{H}_{4}, \mathrm{C}_{2} \mathrm{H}_{6}$, $\mathrm{C}_{3} \mathrm{H}_{6}, \mathrm{C}_{2} \mathrm{H}_{2}$ and $\mathrm{CO}$ at $\mathrm{T}>1000 \mathrm{~K}$ resulting in soot formation.

\section{II.1.3 Coal Swelling}

Coal can swell upon heating resulting in increasing particle size (while drop size remains constant) particularly under pyrolysis in inert environment or reducing conditions. The swelling factor can range from 1.3 (under oxidative) to 4 under in inert environment. A medium value of 1.5 is mostly used for all coals. The swelling increases 
the size by $60 \%$ if pyrolysed in $\mathrm{O}_{2}$ free environment compared to $10 \%$ when heated in $13 \% \mathrm{O}_{2}$ [Street et al., 1969]. Swelling introduces thin walled cenospheres which when burnt can introduce sudden decrease in size and increase in density during burn off.

\section{$\underline{\text { II.1.4 Volatile }}$}

Volatile yields from coal can be as high as $80 \%$ depending upon the type of coal, final temperature and rate of heating. It was found that the volatile yield increased from $30 \%$ at $1250 \mathrm{~K}$ to $63 \%$ at $2100 \mathrm{~K}$ for the same coal [Kobayashi, 1972]. Generally, increased temperature, increased heating rate, decreased particle size, decreased pressure, and reduced bed heights in fluidized beds increase the volatile yields.

Once released, volatiles undergo oxidation in the gas phase. During the volatile combustion period, the gas temperature is much higher than the particle temperatures [Seeker et al., 1981]. The shadow photography of McLean et al. [1981] and the holography of Seeker et al. [1981] reveal that volatiles may burn in jets or as an envelope flame which prevents oxygen from reaching the particle surface for heterogeneous oxidation. Generally speaking, it is difficult to give a single global scheme for volatile oxidation since the volatile are a mixture of combustible gases.

\section{II.1.5 Coal/Char Oxidation Reactions}

\section{II.1.5.1 Heterogeneous Oxidation}

Heterogeneous reactions are generally governed by the following processes: $i$.) Diffusion of species to the particle surface, ii.) adsorption of molecules onto active sites on the surface ( $E=32,000 \mathrm{~kJ} / \mathrm{kmole})$, iii.) formation of the product from the adsorbed 
molecules forming solid oxides on the surface, vi.) desorption of the solid oxides into the gas phase ( $E=170,000 \mathrm{~kJ} / \mathrm{kmole})$, and v.) migration through the boundary layer back into the free stream.

Oxygen transfer to the particles can occur via $\mathrm{O}_{2}, \mathrm{CO}_{2}$, and $\mathrm{H}_{2} \mathrm{O}$, the dominant transfer mechanism being the transfer of diatomic oxygen. The heterogeneous combustion of carbon/char occurs primarily via one or more of the following reactions

$$
\begin{aligned}
& \mathrm{C}+(1 / 2) \mathrm{O}_{2} \rightarrow \mathrm{CO} \\
& \mathrm{C}+\mathrm{O}_{2} \rightarrow \mathrm{CO}_{2} \\
& \mathrm{C}+\mathrm{CO}_{2} \rightarrow 2 \mathrm{CO} \\
& \mathrm{C}+\mathrm{H}_{2} \mathrm{O} \rightarrow \mathrm{CO}+\mathrm{H}_{2}
\end{aligned}
$$

\section{II, 1.5.2 Homogeneous Oxidation}

Two fundamental models were proposed for the char/coal oxidation reactions, single film model and the double film model. The single film model used to model the quasi-steady combustion of coal/char particles assumes that one or a combination of reactions I to IV proceeds at infinite rate with no gas phase reactions (Figure 4a). On the other hand, the double film model assumes that heterogeneous reaction of the particle occurs via reaction III followed by the homogeneous combustion of carbon monoxide to carbon dioxide assuming fast kinetics (Figure $4 \mathrm{~b}$ ). The products of the heterogeneous reactions diffuse out towards the ambient. Due to the high temperature prevailing in the gas phase near the char particle, the combustible gases may react with oxygen in the gas phase which may lower the carbon burning rate. 
a.)

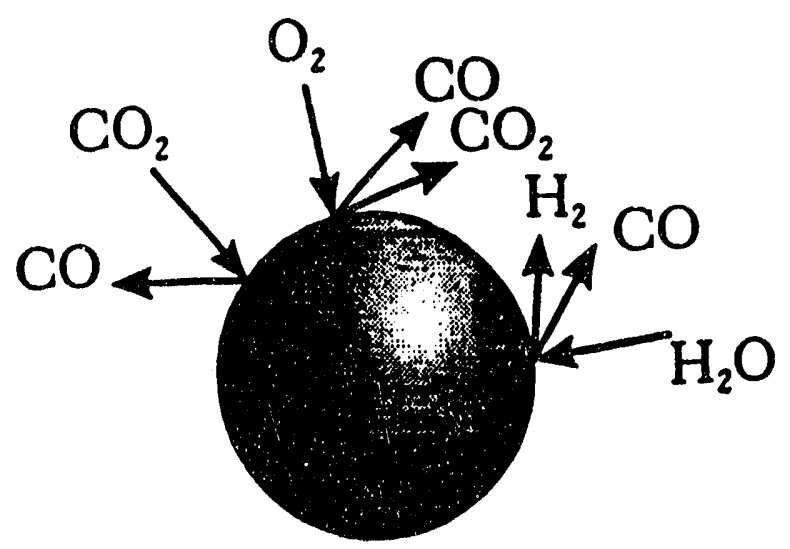

b.)
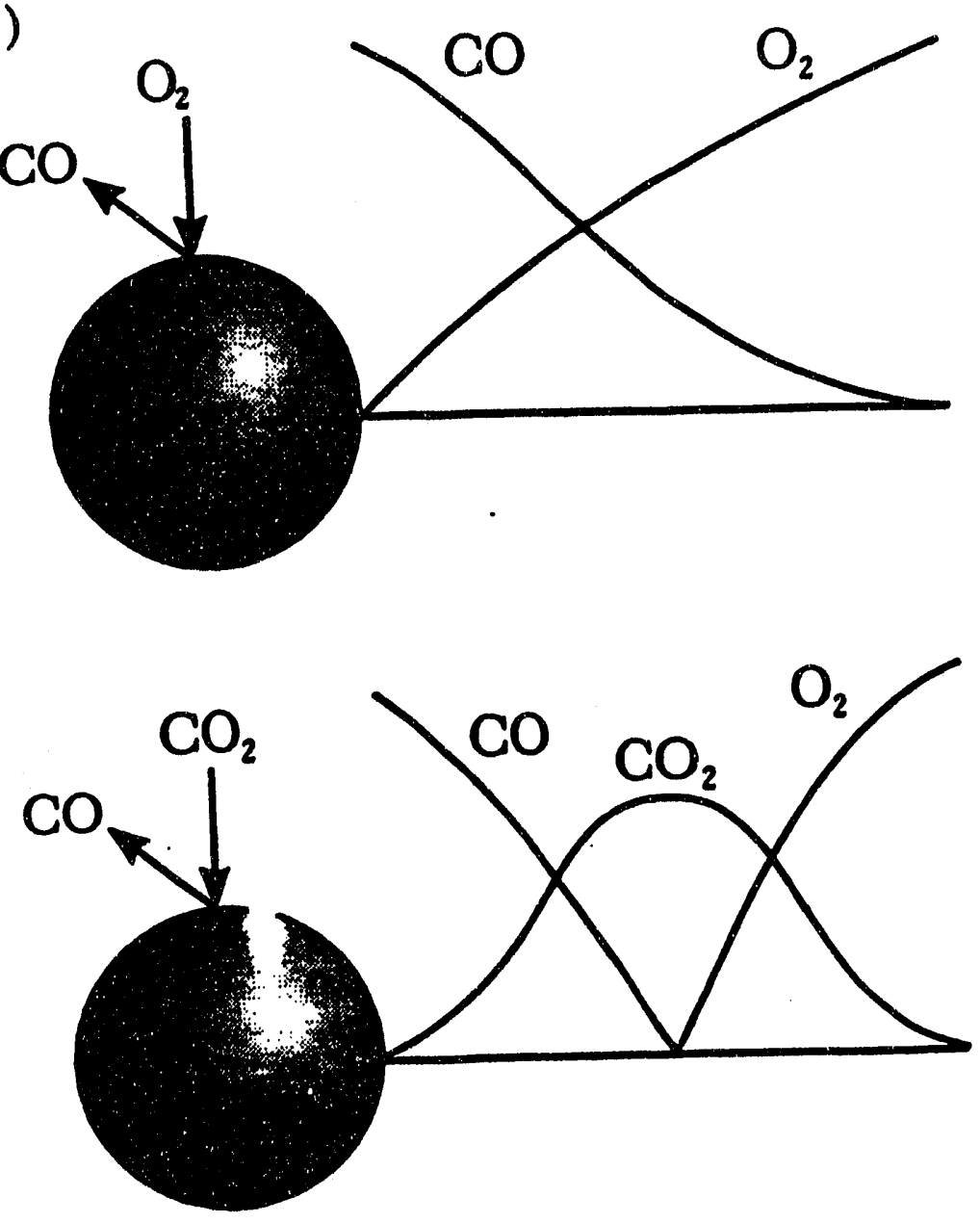

Figure 4. Single and Double Film Models for Char/Coal Combustion 
It is lower compared to the single film model since approximately $50 \%$ of the oxygen diffusing from the ambience is consumed by the CO [Annamalai and Durbetaki, 1977]. II.1.6 Coal Ignition

Carbon particles which are non-gasifying upon heating ignite heterogeneously while oil drops which fully vaporize or a plastic which fully pyrolyzes (e.g. PMMA) ignite homogeneously. Coal, like many polymers, is partially gasifying. Thus, coal could be classified as a charring solid. Volatile matter, flammability of the volatile and transport from the particle determine whether ignition of an isolated coal particle occurs either heterogeneously or homogeneously. If the volatiles evolve earlier, they may remove oxygen from the internal pore surface area preventing heterogeneous ignition. If coal is ignited homogeneously, the volatiles burn in the gas phase (similar to drop flames) preventing oxygen from the particle surface. On the other hand, if combustion is not intense or if volatile liberation occurs as jets from the pores, then oxygen can still reach the particle surface and heterogeneous combustion of fixed carbon and in situ volatile matter can proceed in parallel with gas phase combustion.

Extensive theoretical and experimental studies characterizing char/coal isolated particles ignition and combustion have been conducted in the past. These studies were formulated employing different techniques. Banddyopahyay and Bhaduri [1972] conducted experiments by placing isolated samples of bituminous coals on a Pt wire and immersing them into a hot furnace. Ignition was noticed through an observation port and the minimum gas phase temperatures required for ignition were recorded. They also 
conducted a nonsteady analysis for the ignition of a single coal particle including the radiation heat loss to the environment. The minimum gas phase temperature required for ignition was determined.

Karcz et al. [1980] studied the ignition of a bituminous coal, an anthracite, and a char produced from the bituminous coal. Single particles were placed on the tip of a fine quartz needle and introduced into a hot furnace. They found that the coal particles ignited heterogeneously for small particle diameters and homogeneously for larger particle diameters. Using Semenov's ignition theory and their experimental results for small particles (heterogeneous ignition), they determined activation energies and preexponential factors for fuels tested. The activation energies ranged from 46,300 $\mathrm{kJ} / \mathrm{kmole}$ to $103,400 \mathrm{~kJ} / \mathrm{kmole}$.

They also determined the ignition temperatures of single coal particles. The particles were placed on the tip of a fine quartz needle and if ition was observed through an optical window. While this technique is similar to the experimental technique of Bandyopahyay and Bhaduri [1972], a quartz needle was used instead of a Pt needle which can catalytically affect the ignition process. The experimental data for $T_{i} v s d_{p, 0}$ is shown in Figure 5 for anthracite and bituminous coals and chars.

Karcz and Zembrzuski [1982] obtained thermographs (recording of infrared radiation between $2-5.6 \mu \mathrm{m}$ ) of $500 \mu \mathrm{m}$ diameter anthracite and lignite particles. The particles were placed on a quartz needle and suddenly exposed to a combustion chamber at $1023 \mathrm{~K}$. Ignition was said to occur when $T_{p}$ exceeded $T_{\infty}$ and $T_{p}$ continues to increase 


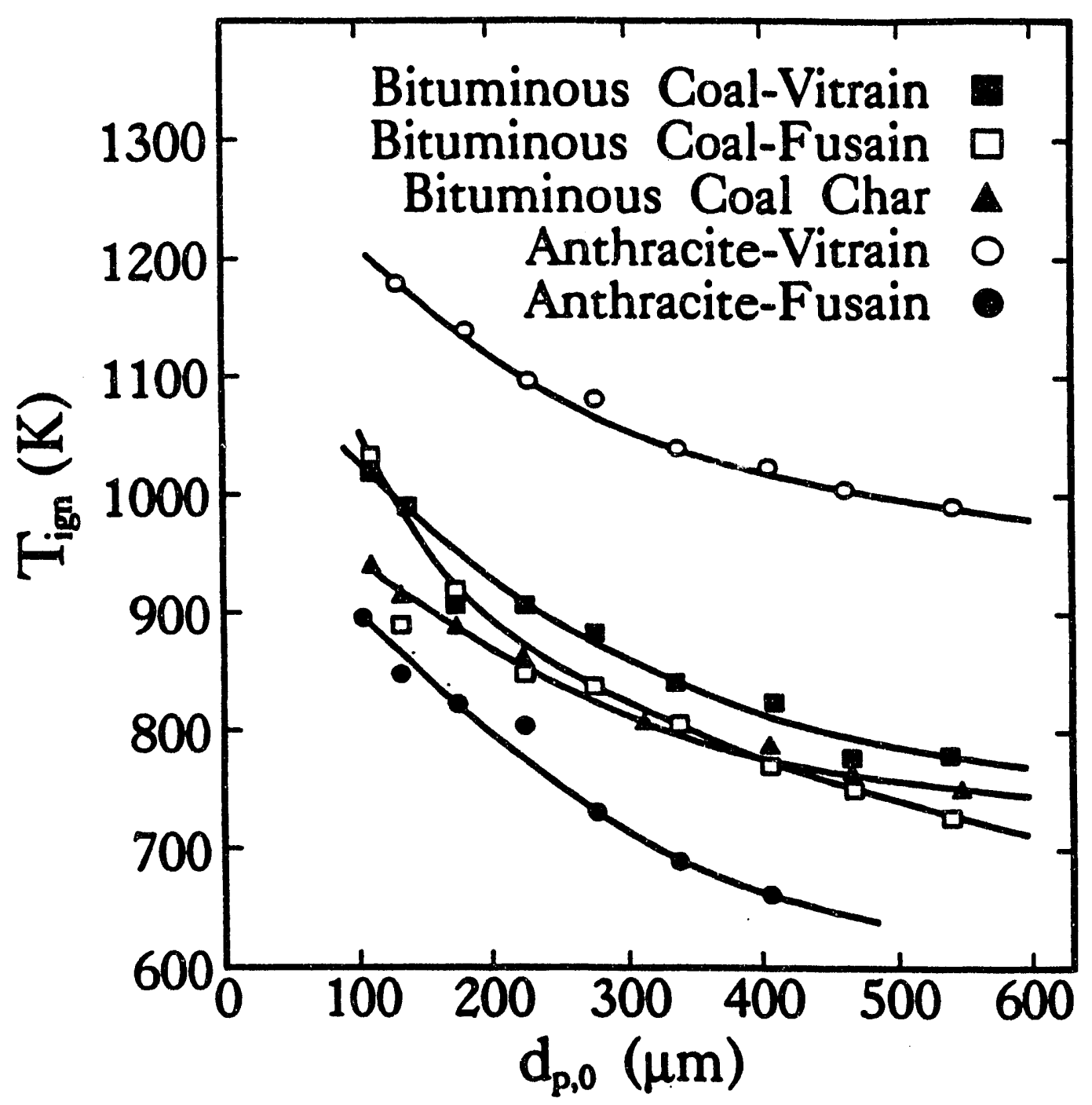

Figure 5. Ignition Temperature Variation of Coal and Anthracite Particles 
For anthracite, the ignition time is $2 \mathrm{sec}$. while for lignite the volatile ignited at about $0.8 \mathrm{sec} .\left(\mathrm{T}_{\mathrm{p}}\right.$ is about $\left.800 \mathrm{~K}\right)$. The anthracite particles heated to about $1000 \mathrm{~K}$ in $1 \mathrm{sec}$. after which time the particle temperature remained constant for about $1 \mathrm{sec}$. and then heated very rapidly again. Qualitatively, this behavior is similar to the behavior of the model results of Bandyopahyay and Bhaduri. Heterogeneous ignition occurred at about 2 sec. since $T_{p}$ rose rapidly above the gas temperature at this time. The authors claim that the ignition temperature of the anthracite particle is about $963 \mathrm{~K}$, which is much lower than the ignition temperature reported by other researchers. The difference was attributed to the experimental technique for measuring particle temperature; however, it should be noted that the relatively large particle size may have also been responsible.

Tomeczek and Wojcik [1990] measured the ignition temperatures of particles directly by placing the particle on a heating element ( $\mathrm{NiCr}-\mathrm{Ni})$ which also serves as a measuring junction. Pulsed heating $(1 \mathrm{kHz})$ is used. The temperature was measured during the "off" timing of the pulse and ignition was recorded by observing the luminous flux from the particle. Five different sizes $(0.1-1 \mathrm{~mm})$ were used in 10 to $100 \%$ $\mathrm{O}_{2}$ concentrations. As opposed to previous work, which assumed $T_{z}=T_{p}$ at the time of ignition, this technique directly measured $T_{p}$. The authors report a value of 120,000 $\mathrm{kJ} / \mathrm{kmole}$ for activation energy, with $\mathrm{n}=1$ for char and 0.5 for anthracite. Char had a lower $T_{p, i g n}(900-1000 K)$ than anthracite $(1100-1400 \mathrm{~K})$ for $d_{p}$ ranging from 0.2 to $1 \mathrm{~mm}$. While experimental methods which involve suspending a particle from a fiber result in an additional conductive heat loss from the particle through the fiber, the 
experimental technique of Tomeczak and Wojcik minimize this loss since the heat which is transferred through the wires is removed from the heating element rather than the particle. The authors believe that Semenov's theory is not applicable for coal particle ignition since it is a partially pyrolyzing solid.

Annamalai and Durbetaki [1977] analyzed the ignition of coal particles assuming (i.) heterogeneous reactions in the absence of homogeneous reactions and (ii.) homogeneous reactions in the absence of heterogenous reactions. For case (ii.), reactions were assumed to occur at a location where the volatile concentration was in stoichiometric proportion to oxygen. Initially, the ambient supplies heat to the particle since the gas phase is almost frozen. As the ambient temperature is increased, the reaction rate increases and a condition is reached at which $d T / d r$ at the reaction zone is zero $\left(T_{R}>T_{p}\right)$ and thus the reaction zone supplies the enthalpy required for pyrolysis and to raise the temperature of the volatile to the reaction zone temperature while the heat transferred from the ambience is zero. This criterion called adiabatic ignition criterion. They determined $T_{i g}$ vs $Y_{02, \infty}$ and $d_{p, 0}$. Figure 6 shows the variation of ignition temperature for gas (homogeneous) ignition and carbon (heterogeneous) ignition with particle size. For homogeneous ignition (GIT), it is seen that decreasing particle size increases $\mathrm{T}_{\mathrm{iga}}$ as in the heterogeneous mode (HIT). However, for homogeneous ignition, increased oxygen concentration results in increased values of $T_{\text {iga. }}$.

Howard and Essenhigh [1966] estimated that smaller particles $(<65 \mu \mathrm{m})$ can not produce volatile at a fast enough rate in order to prevent all the oxygen from reaching 


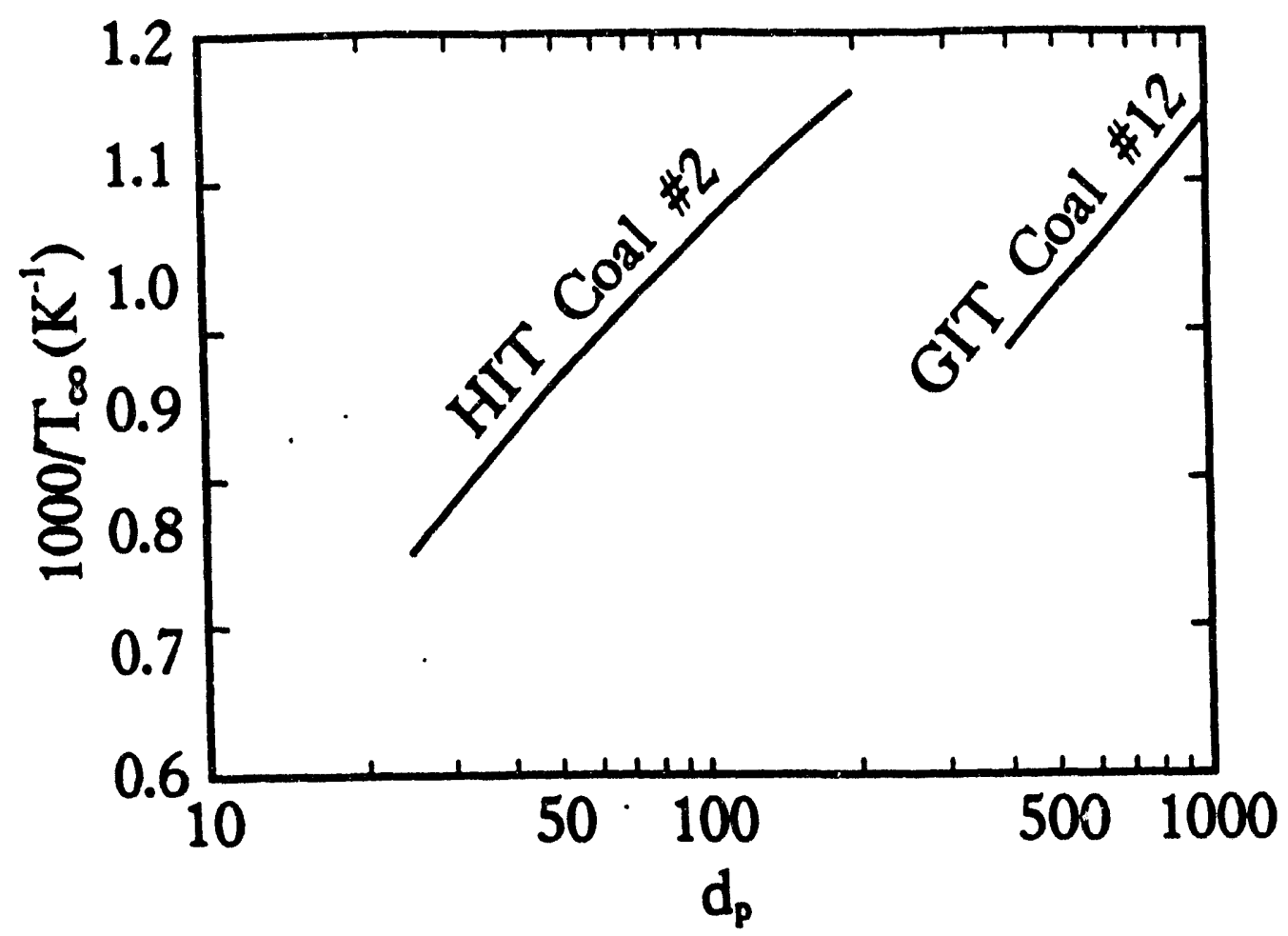

Figure 6. Variation of Ignition Temperature for Gas (Homogeneous) Ignition and Carbon (Heterogeneous) Ignition with Particle Size 
the particle surface. Thus, both heterogeneous and homogeneous combustion may proceed in parallel. Further, for $\mathrm{d}_{\mathrm{p}}<15 \mu \mathrm{m}$ only heterogeneous reactions are present. In support of their arguments they found that the activation energy during simultaneous heterogeneous and homogeneous combustion to be $50,000 \mathrm{~kJ} / \mathrm{kmole}$ indicating adsorption control (due to reduced $\mathrm{O}_{2}$ at the particle surface) while nearer to burn out, the activation energy jumped to $250,000 \mathrm{~kJ} / \mathrm{kmole}$ indicating desorption control.

Solomon et al. [1990] conducted ignition experiments for high and low rank coals. Hot air $\left(T_{\varepsilon}=850 \mathrm{C}\right)$ was injected vertically upward through a screen and coal particles (lignite, subbituminous and bituminous) were entrained through a $4 \mathrm{~mm}$ tube located centrally within the reactor. From short exposure photographs, the particle velocity was estimated to be $2.5 \mathrm{~m} / \mathrm{s}$ near the injector and $4.8 \mathrm{~m} / \mathrm{s}$ downstream within the flame. The temperature of burning particles is about $750 \mathrm{C}$ higher than the gas temperature $\left(T_{p}=1900\right.$ to $\left.2010 \mathrm{~K}\right)$. In order to elucidate the ignition control, experiments were performed both in air and $N_{2}$. The temperature at which $10 \%$ of the initial coal mass was lost were measured for both the oxidizing and non-oxidizing environments. If the temperatures were the same for both cases, then the majority of mass loss occurred due to pyrolysis and ignition was assumed to occur by homogenous control. On the other hand, if the temperatures were different, then a portion of the mass loss in the oxidizing atmosphere must have occurred by heterogeneous reactions, thus indicating both heterogeneous and homogeneous ignition control. These experiments indicate that high rank coal ignited under homogeneous control, while the low rank coal 
exhibits both homogeneous and heterogeneous behaviors.

Holographic studies we conducted on 38-100 $\mu \mathrm{m}$ lignite and $40 \mu \mathrm{m}$ bituminous particles in oxygen mass fractions of 3-10\% by Seeker et al. [1981]. These studies reveal the presence of a spherically symmetric soot-like cloud around the particle as a result of volatile oxidation in the gas phase. Smaller particles reach much higher particle temperatures than larger particles due to their low thermal inertia and high temperature gradients between the particle and the volatile flame. If the particles had been combusting heterogeneously, the opposite would be expected.

Timothy et al. [1982] obtained two color pyrometry traces of burning lignite and bituminous coal particles for two size ranges (38-45 and 90-105 $\mu \mathrm{m}$ diameters), temperatures between 1250 and $1700 \mathrm{~K}$ and oxygen mass fraction of $15 \%$ to $100 \%$. The experiments were conducted in a $5 \mathrm{~cm}$ diameter muffle tube furnace. The volatile are believed to form a soot-like cloud around the particles in agreement with the results of Seeker [1981].

\section{II.2 Combustion of Isolated Char/Coal Particles}

\section{II.2.1 Coal Combustion}

Once a single oil drop is placed in a hot furnace (Figure 3a), enthalpy is supplied to the drop from the ambience. Vapors released by the drop move radially into the ambience where they mix with oxygen and establish a diffusion flame away from the droplet surface. The vaporization of the fuel drop is a purely physical process, in that, 
the molecular composition of the fuel in the gas phase is the same as the drop molecular composition. On the other hand, coal particles partially pyrolyze releasing volatiles (chemical decomposition of the particles to the gaseous hydrocarbon species) and leaving char particles (mostly carbon). The volatiles diffuse into the surrounding atmosphere where they mix with oxygen and a flame is formed if the volatiles exist in sufficient proportions. After the volatiles are exhausted, only char (carbon and hydrogen in small amounts) is left behind (Figure 3b). The flame established by the volatile extinguishes and now oxygen diffuses to the char surface and reacts to form carbon monoxide (Figure 3c) and carbon dioxide (heterogeneous ignition). It should be noted that unlike the combustion of volatiles, which diffuse towards the oxygen rich atmosphere resulting in a large reaction area, for heterogeneous combustion the oxygen must be transported to the particle surface. Results of previous researchers have shown that large particles with a high volatile content ignite homogeneously while smaller particles ignite heterogeneously [Howard and Essenhigh, 1966; Annamalai and Durbetaki, 1977]; however, a cloud of small particles, which would ignite heterogeneously if isolated, can ignite homogeneously if placed closely together in sufficient concentration to form a flammable mixture in the gas phase around the cloud [Ryan and Annamalai, 1991].

\subsubsection{Char Combustion}

Char is essentially a porous solid (mostly carbon) with a large internal reaction area. Since FC is dominant, it produces the majority of the heat released during combustion. During char combustion, $\mathrm{CO}_{2}$ and $\mathrm{CO}$ are formed due to heterogeneous 
reactions and diffuse out towards the ambience. Due to the high temperature prevailing in the gas phase near the char particle, the carbon monoxide reacts with the oxygen in the gas phase to produce a faint blue color flame. It should be noted that as oxygen diffuses from the ambience towards the particle surface, it penetrates inside the particle and reacts on the internal surfaces. Thus, carbon/char particles burn internally with decreasing density and/or externally (thin layer) with decreasing diameter. Char combustion is first considered to be pure carbon with reactions at the external surface area of particle and then the porous nature of the char particles are considered.

Since the chemistry of carton is relatively well defined compared to coal and coal pyrolysis, earlier fundamental studies were concentrated on ignition and combustion of carbon particles. The classical works of Tu et al. [1934] deal with the combustion of carbon spheres ( $2.54 \mathrm{~cm}$ diameter) in $\mathrm{N}_{2}-\mathrm{O}_{2}$ mixtures for gas phase temperatures ranging from 935 to $1712 \mathrm{~K}$ covering kinetically controlled and diffusionally limited combustion. They suspended the particles within a refractory tube and rotated the particles to simulate relative velocities up to $50 \mathrm{~cm} / \mathrm{s}$. The particle mass was recorded as it burned. In order to interpret their results, they developed a model based on the resistance concept. The model considers diffusion (diffusional resistance) through the boundary layer followed by chemical reactions (chemical resistance) at the particle surface with the primary products being $\mathrm{CO}$ and $\mathrm{CO}_{2}$. They found that for $\mathrm{T},>1100 \mathrm{~K}$, combustion is diffusion controlled.

Spalding [1951] considered quasi-steady combustion of a carbon particle in an 
ambient temperature of $300 \mathrm{~K}$ assuming infinite heterogeneous oxidation kinetics. $\mathrm{He}$ presented two separate models: (i.) assumes heterogeneous production of $\mathrm{CO}_{2}$ with no dissociation in the gas phase and (ii.) with the heterogeneous production of $\mathrm{CO}$ and $\mathrm{CO}_{2}$ with equilibrium of carbon dioxide in the gas phase. He found that $T_{p}$ for case (i) is $2700 \mathrm{~K}$ while for case (ii) $T_{p}=1730 \mathrm{~K}$ with $Y_{02, \infty}=0.23$. The ratio of the heterogeneous production rates of $\mathrm{CO}$ and $\mathrm{CO}_{2}$ in case (ii.) is given by thermodynamic equilibrium. The burning rate for case (i.) is half the burning rate of case (ii.). On the other hand, one can consider a third case of heterogeneous $\mathrm{CO}$ production and frozen gas phase, then the burning rate and particle temperature are identical [Annamalai and Caton, 1987].

\section{IL.3 Interactive Combustion of Char/Coal Particles}

While the literature on droplets arrays is extensive [Annamalai and Ryan, 1992], the literature on two-particle arrays is very limited.

Gieras et al. [1986] conducted experimental and theoretical investigations into the ignition and combustion processes of coal particles arranged in a row under zero and normal gravity conditions. The influence of particle diameter $(0.3-1.2 \mathrm{~mm})$, coal volatile content $(2-48 \%)$, and oxygen concentration in the gas phase (21-100\%) on the ignition process and the rate of flame propagation were explored. A physical model of the process was presented to explain the flame propagation mechanism and determines the ignition requirements. On the basis of this model, a mathematical model of the process 
was formulated which predicts the influence of various heat transfer mechanisms on particle ignition and flame propagation.

Zero gravity experiments were performed using a free fall method. Two coal particles were arranged in horizontal and vertical arrays by attaching them to quartz needles. A spark ignited n-octane droplet was used as the ignition source with the distance from the coal particle array chosen to minimize the influence on the combustion of the array. Figure 7 shows the flame propagation between two brown coal particles in the horizontal configuration $\left(d_{p}=0.7 \mathrm{~mm}, 1=4.1 \mathrm{~mm}, 30 \% \mathrm{VM}, 70 \% \mathrm{O}_{2}\right.$ and $\mathrm{N}_{2}=30 \%$ ). At about $700 \mathrm{~ms}$ after ignition of the first coal particle, the second particle has ignited. The oxygen between the particles is then depleted and a common flame is formed around both particles.

They also considered upward propagation (vertical array, bottom particle ignited first), downward propagation (top particle ignited first) and horizontal propagation under conditions of normal gravity. Figure 8 shows the ignition sequence for the horizontally aligned particle array under normal gravity conditions. The maximum ignition distance was denoted as the maximum particle separation distance for which flame propagation could occur between the two particles. Maximum ignition distance was approximately the same for the cases of horizontal and downward propagation. However, for the case of upward propagation, the maximum ignition distance increased since heat released by the lower particle is convected to the upper particle. In fact, the maximum ignition distance was such that the particle flames never overlapped during combustion, however, 


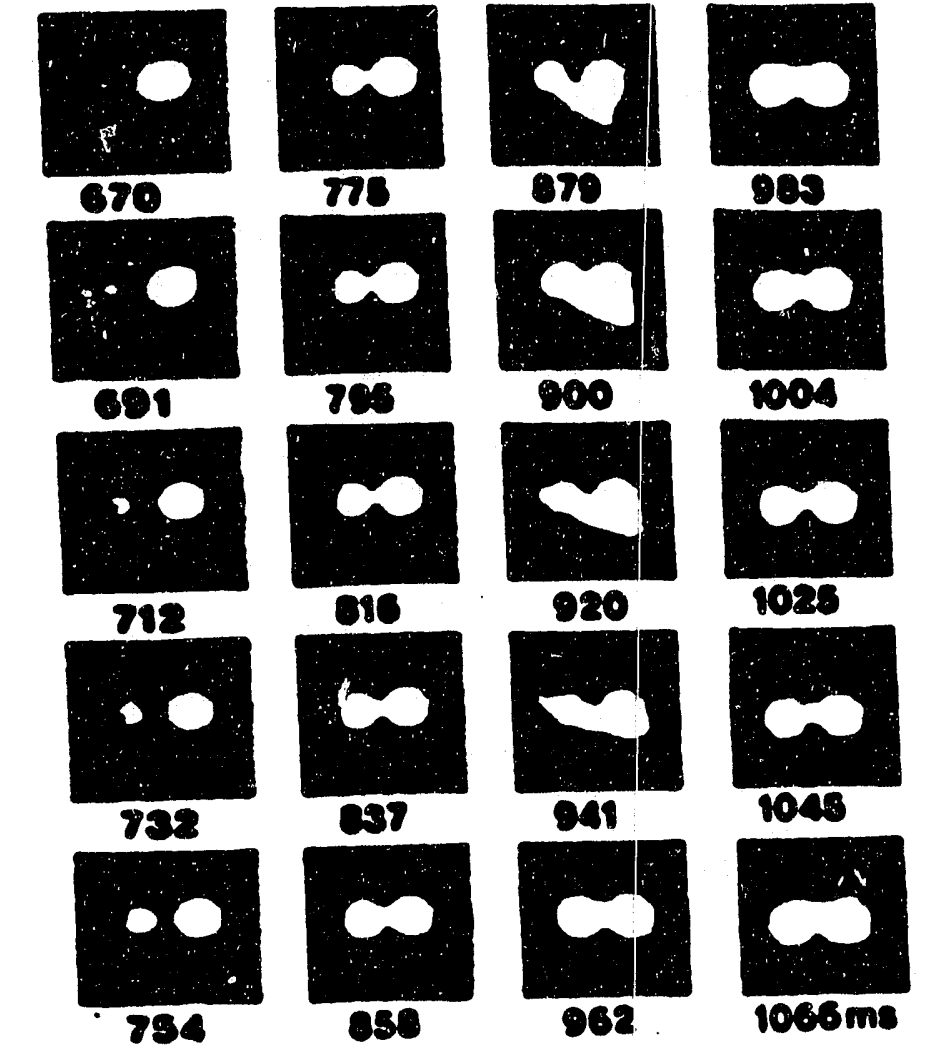

Figure 7. Ignition at Zero Gravity for a Binary Coal Particle Array 
4

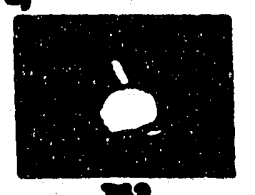

(2)

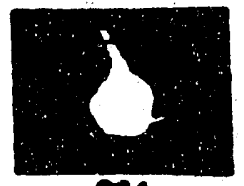

n

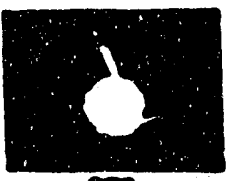

is

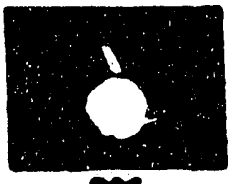

m

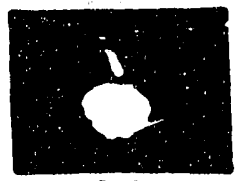

onsm v

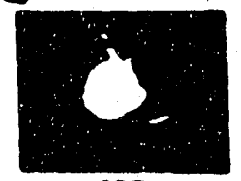

$\infty$

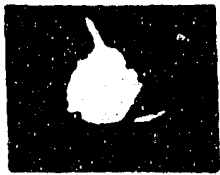

$\pi$

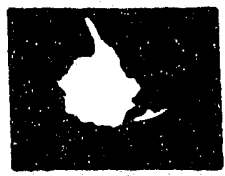

70

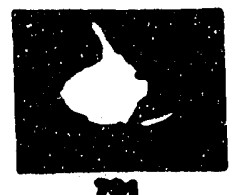

m

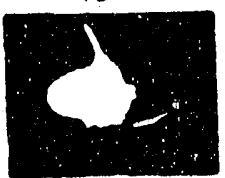

02000

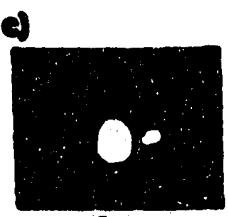

80

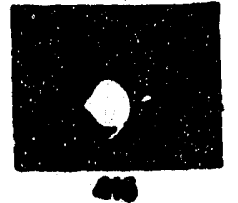

cis

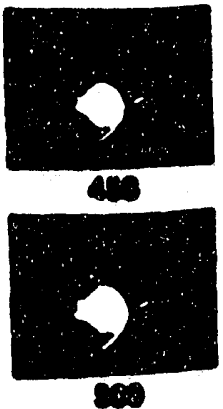

$\infty$

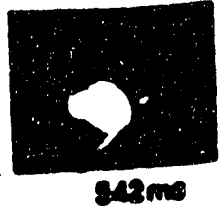

Figure 8. Ignition at a Normal Gravity for a Binary Coal Particle Array 
the array took longer to burn than the other two configurations since the upper particle burns in the wake of the combustion gases from the lower particle. For a given alignment, oxygen mass fraction and coal volatile mass fraction seem to be the dominant factors determining the maximum ignition distance. They determined the average burning rate constant, $\alpha$, simply as $d_{p}^{2} / t_{b}$. At normal gravity in the horizontal configuration, the average burning rate constant was about $8 \%$ greater than the average burning rate constant under zero gravity conditions since free convective effects supply additional oxygen to the particles.

The authors estimated the flame propagation velocity by dividing the interparticle separation by the flame transfer time for each configuration while varying interparticle separation (Figure 9). Their results show that there is an optimum interparticle separation at which the flame transfer rate is a maximum for each geometry. Generally, the flame propagation velocity increases with increasing oxygen concentration, volatile matter, and decreasing particle diameter.

\section{II.4 Image Processing Applications in Combustion}

It is apparent from the previous theoretical studies that a group flame is likely to be formed at closer interparticle spacing. However, there are no experimental data which shows the sequences of events leading to group flame. The emerging image processing technology has provided viable means for image acquisition of ignition and combustion sequence leading to group combustion phenomena. 


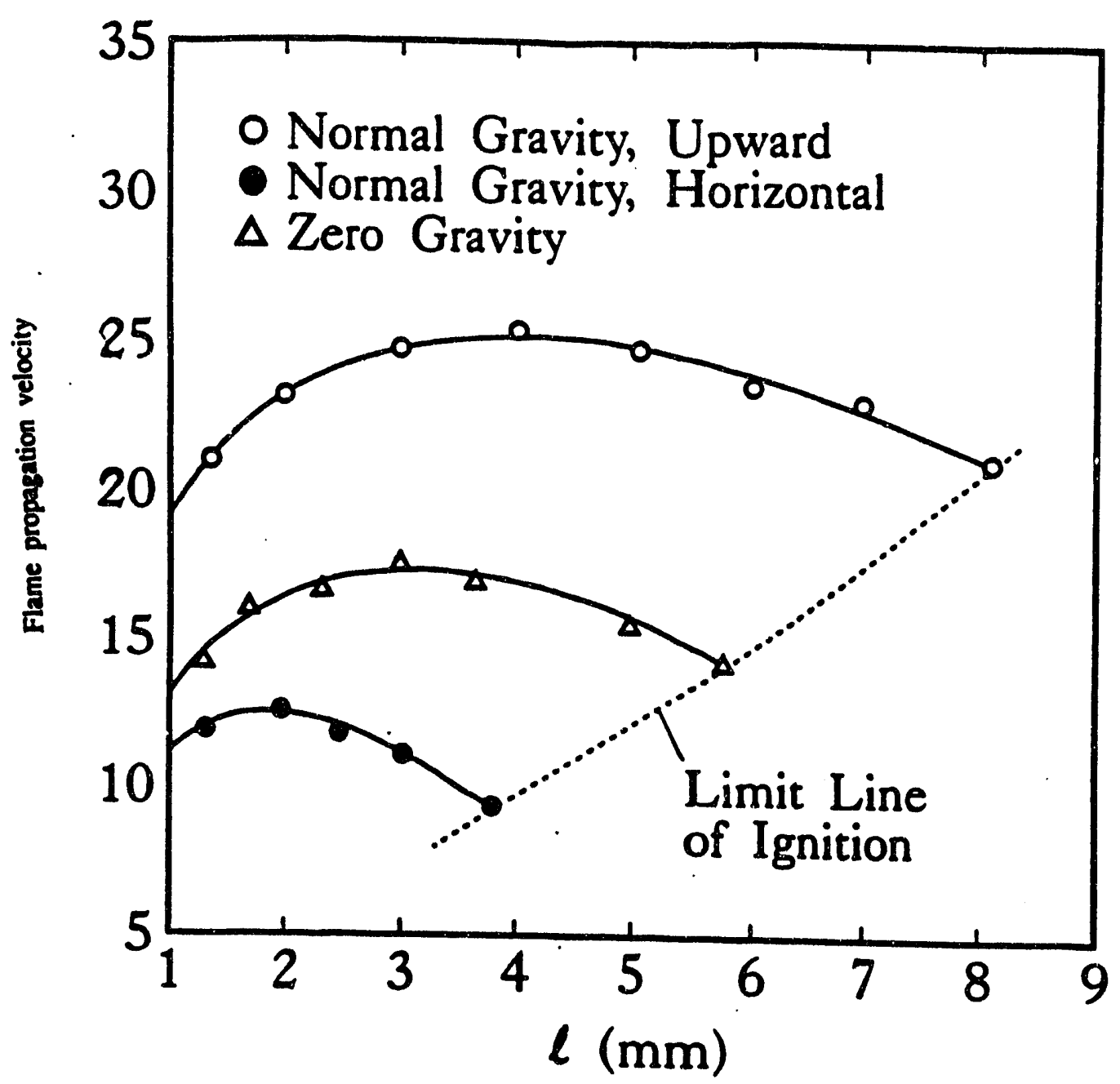

Figure 9. Flame Propagation Velocity vs Interparticle Separation Distance 
From the beginning of science, visual observation has played a major role. At that time, the only way to document the results of an experiment was by verbal description and manual drawings. The next major step was the invention of photography which enabled results to be documented objectively. Three prominent examples of scientific applications of photography are astronomy, photogrammetry, and particle physics. Astronomers were able to measure positions and magnitudes of stars accurately. Aerial images were used to produce topographic maps. These manual evaluation procedures, however, were time consuming. Some semi- or even fully automated optomechanical devices were designed. However, they were adapted to a single specific purpose. This is why quantitative evaluation of images never found widespread application at that time. Generally, images were only used for documentation, qualitative description and illustration of the phenomena observed.

Nowadays, in the middle of a second revolution sparked by the rapid progress in video and computer technology, personal computers and workstations have become powerful enough to process image data. They have also become cheap enough to be widely used. In consequence, image processing is turning form being a specialized science in areas such as astronomy, remote sensing into a standard scientific tool. Since pictorial information provides much more details, a fact which can be precisely summarized by the saying that "a picture is worth a thousand words", applications in image processing have now been applied to virtually all the natural sciences.

The literature review, conducted on image processing applications in combustion, 
revealed vast amount of studies. Some of these studies are given here.

Direct visual observations of a high Reynolds number jet, employing digital image processing techniques, were obtained by Mungal and Hollingsworth [1989]. The jet consisted of the exhaust plume of a TITAN IV rocket motor, which was discharged upward during ground-based testing producing an estimated Reynolds number of about $2 \times 10^{8}$. Image processing is used to enhance the plume appearance and reveal significant events associated with the jet evolution. The process involved two firings, the first under overcast conditions and the second under clear, sunny skies. Both firings were recorded on $3 / 4$ in. videotape with a sony CCD video camera (Description of CCD cameras are given later in chapter IV) at 30 frames per second. The entire videotape of the first firing covered the first $600 \mathrm{ft}$ downstream from the jet exit, consisting mostly of the bright after burning plume. For the second firing, the video tape covered the first 2000 $\mathrm{ft}$ of the plume from the time of ignition to approximately $1 \mathrm{~min}$ into the burn. In the remaining $1 \mathrm{~min}$ the camera is moved to capture the bright exhaust flame, and so does not show the bulk of the plume.

The most striking finding was the progression of organized structures up through the jet, similar to those observed in laboratory flew at Reynolds numbers of $10^{4}$.

Planar Imaging has been used to investigate the mixing and soot formation characteristics of a subscale gas generator multi-element triplet injector, 2.18 inches in diameter with 18 impinging triplet elements distributed in an annular pattern [Makel and Kennedy, 1991]. Fuel was injected through two of the orifices (36 total locations) set 
at a 30 degree angle. The oxidizer is injected up the center orifice of each element. The inner and outer fuel elements are oriented to generate a swirl flow in opposite directions with the aim of enhancing mixing (compared to radially aligned impinging elements). A vertically polarized laser beam (532 $\mathrm{nm}$ wavflength) from a frequency doubled Nd:YAG laser is formed into a thin sheet $(0.20 \mathrm{~mm}$ thick). The short laser pulse froze the turbulent flow field and allowed instantaneous images of the smoke particles to be obtained. For the mixing experiments the simulated oxidizer flow entered a smoke generator where it was mixed with smoke from burning incense and then passed through a set of fine mesh screens to remove any entrained ash and large particles which do not follow the flow. The scattered laser light from the smoke aerosol was collected at 90 degrees to the laser sheet and was imaged with a camera lens onto a standard RS-170 CCD camera with a resolution of 512 by 240 pixels and with a framing rate of $60 \mathrm{~Hz}$. The video images were acquired and then digitized by an eight bit $A / D$ frame grabber (Imaging Technology Vs-100) board and transferred to an Apollo DN10000 computer workstation.

It is apparent from the review that while extensive theoretical studies were conducted in combustion, and limited applications of video imaging on rocket plumes and fuel injectors, there is no study on the application of digital image processing to isolated and interactive combustion. The present research study deals with image processing applications in the ignition and combustion of isolated char/coal particles and a binary array of char/coal particles. 


\section{CHAPTER III}

\section{OBJECTIVES}

The primary objectives of this work were:

(i.) to develop a digital image processing system and to enable it's operation,

(ii.) to apply the image processing techniques in the current research study.

(iii.) to conduct preliminary experiments on the combustion of isolated miniature candles as well as on the combustion of two-candle arrays, and

(iv.) to conduct experimental studies employing digital image processing techniques in determining the ignition and combustion characteristics of isolated char/coal particles as well as two-particle char/coal arrays.

The results of this work are expected to provide fundamental understanding of the ignition and combustion characteristics of isolated char/coal particles as well as interacting char/coal particles and to provide qualitative information on them. 


\section{CHAPTER IV}

\section{OVERVIEW OF DIGITAL IMAGE PROCESSING}

An image, in its strict definition, is a representation of an object consisting of descriptive information about that object. Extracting sophisticated data and useful information from that image requires the use of computers. While most people are familiar with photographic or pictorial images, computers, however, work with numerical rather than pictorial information. Thus, in order to manipulate images by computers, an image must be converted first into numerical form by digitization.

In digitization, an image is divided into a horizontal grid of small regions containing picture elements, or pixels. A pixel is an abbreviation of the word picture element. The image is represented by this digital grid, or bitmap, and each pixel has an address (row number and column number) in the bitmap (Figure 10). Each pixel has a value associated with it which describes it in terms of brightness, contrast, and color or shades of gray in the case of a gray-scale image (from black, through shades of gray, to white). The largest number of distinct shades of gray is 256 . Thus, each pixel in a gray-scale image may have any value from 0 (pure black) to 255 (pure white), and these values are what are manipulated in image processing. Gray scale images are analogous to black and white photographs; the brightness and darkness of the image varies smoothly over the image.

The quality of a digital image depends on two characteristics: spatial resolution 


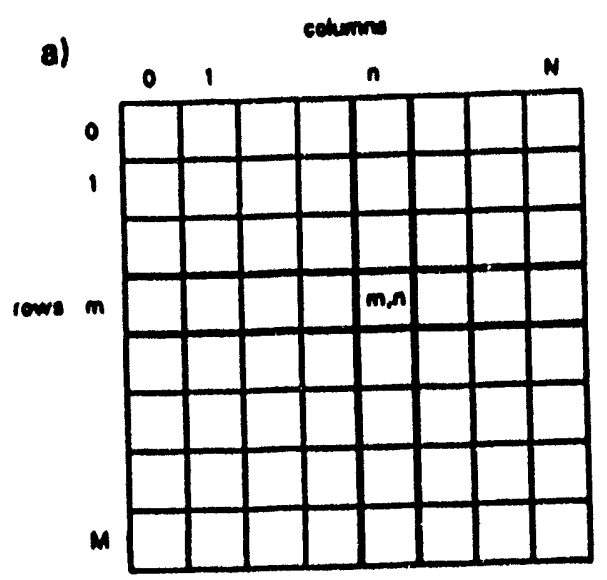

b)

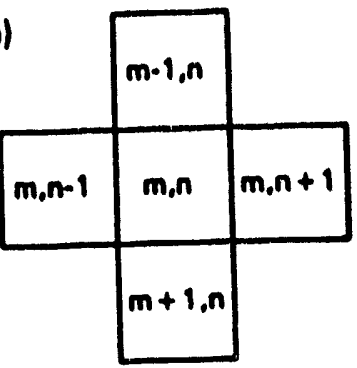

4-Neighborhood c)

\begin{tabular}{|l|l|l|}
\hline$m-1, n-1$ & $m-1, n$ & $m-1 n+1$ \\
\hline$m n-1$ & $m n$ & $m n+1$ \\
\hline$m+1, n-1$ & $m+1, n$ & $m+1 n+1$ \\
\hline
\end{tabular}

Figure 10. Illustration of a Digital Grid (Bitmap) 
and depth. Spatial resolution refers to the height and width of an image in pixels, while depth describes the number of distinct shades of gray (or colors) each individual pixel can have.

Higher spatial resolution generally translates into higher quality images. For example, an image of $512 \times 512$ pixels (512 pixels wide and 512 pixels high) has a higher quality than an image of $248 \times 248$ pixels. Since the width and the he,ght are divided more finely (512 divisions versus 248 divisions), the image can be more finely represented.

Digital image processing requires images to be obtained in the form of electrical signals, sampling. These signals can be digitized into sequences of numbers which then can be processed by a computer. There are many ways to convert images into digital numbers. Here, the focus will be on video technology, the most common and affordable approach, since it is the method employed in this research project.

\section{1 Components of a Digital Image Processing System}

In this section, the technical innovations that enabled the widespread application of image processing in science are briefly discussed. It will outline the capabilities of modern image processing systems and the progress in image sensors, image storage, and image processing.

\section{IV.1.1 Image Sensors}

The milestone in image sensing technology was the invention of semiconductor 
phtodetector arrays. There are many types of such sensors, the most common being the solid state charge coupled device (CCD). In a CCD camera the sensor is partitioned into discrete, light sensitive elements, arranged in horizontal rows and vertical columns (2dimensional array). The number of elements in the sensor determines the camera resolution. During the image capturing phase, each element collects electrical charges, which are generated by absorbed photons. Thus the collected charge is proportional to the illumination. In the read-out phase, these charges are converted to an electric voltage forming an analog signal. The signal leaving the camera's sensor is amplified inside the camera. This signal is transferred over a coaxial cable to the frame grabber.

While CCD design objectives aimed primarily at developing low cost "picturemaking" products for the home video and commercial security markets, charge injection device (CID) sensors development took a different track. Targeting system automation, CID design objectives focused on achieving complete, accurate, and distortion-free image data acquisition for computer processing with fast, flexible timing and function control.

The CID structure, principle of operation, and readout techniques are fundamentally different from CCD's, providing useful performance advantages. While CCD's transfer the collected charge out of the pixels during readout (hence erasing the image stored on the sensor), CID's do not. Instead, readout is accomplished by determining the charge's value and transferring it out instead of the charge itself. Thus, readout is non-destructive because charge remains intact in the pixel after the signal level 
has been determined.

Semiconductor imaging sensors have a number of significant advantages:

i.) Precise and stable geometry: this feature simply results from the manufacturing procedure. Geometric distortion is virtually absent. More important, the sensor is stable in position, showing only a minor temperature dependence due to the low linear thermal expansion coefficient of silicon $(2.1 \times 0-6 / \mathrm{K})$. These features allow precise size and position measurements.

ii.) High sensitivity: the quantum efficiency, i. e., the fraction of elementary charges generated per photon, is close to one. However, commercial CCDs cannot be used at low light levels because of the thermally generated electrons. But if CCD devices are cooled down to low temperatures, they are among the most sensitive imagers.

iii.) Size: a final advantage is the small size of the sensor and its insensitivity to external influences such as magnetic fields and vibration.

\section{IV.1.2 Image Storage}

Images contain huge amount of data. A standard image from a $35 \mathrm{~mm}$ camera measures $24 \mathrm{~mm} \mathrm{X} 36 \mathrm{~mm}$. If a resolution of $0.01 \mathrm{~mm}$ is assumed, it consists of more than $10^{7}$ data points (pixels). Each pixel needs several bits to resolve the different gray values of the image (maximum of 256). One image pixel can be stored in one byte ( 8 bits). The whole image of $10^{7}$ pixels would occupy 10 Mbytes. A color image would require three times as much space since three color channels, red, green, and blue, must be stored. 
Most images, now processed, are captured by video cameras which provide a much lower resolution. A widespread standard contains $512 \times 512$ image pixels. One gray-scale image with 8 bits/pixel contains 256 kbytes of data.

However, applications which analyze time varying processes cannot be studied with single frames, but require the analysis of image sequences. The storage requirements then increase tremendously. A single second of video images with 30 frames/s needs 7.5 Mbytes of storage. Three-dimensional imagery, which can really adequately picture the three-dimensional world, also needs huge storage space. A single $512 \times 512 \times 512$ image occupies 128 Mbytes.

Because of the immense amount of data in images, successful image processing requires iarge computing power. A current personal computer is about as powerful as a main frame ten years ago and sufficiently fast to perform not too complex image operations.

Complex operations and image sequence analysis, however, need more processing power. These demands can also be met with current PC-based systems, which are equipped with image processing hardware, frame grabbers, for specific operations.

\section{IV.1.3 Image Processing}

The term image processing is used to mean a variety of things, from simply enhancing the visual appearance in an image to extracting statistical data from an image using sophisticated analysis techniques. All image processing is performed by 
mathematically operating on the values of the pixels. In the broadest sense, it is applying mathematical functions such as matrix multiplication, adidition, etc.., that will improve the quality or clarity of an image.

\section{IV.1.3.1 Hierarchy of Image Processing Operations}

Image processing is not a one-step process. It is essential to distinguish between several steps which must be performed one after the other until the data of interest from the observed scene is extracted. In this way a hierarchical processing scheme is built up as shown in Figure 11. An overview of the different phases of image processing is given below.

Image processing begins with the capturing of an image with suitable, not necessarily optical, acquiring system (image acquisition \& formation). Then the image sensed must be converted into image points on a discrete grid. This puts it into a form which can be treated with digital computers. This process is called digitization.

The first steps of digital processing may include a number of different operations. It may be necessary to correct known disturbances in the image, for instance caused by a defocused optics, motion blur, errors in the sensor, or errors in the transmission of image signals (image restoration). If the sensor has non linear characteristics, these need to be corrected. Likewise, brightness and contrast of the image can be optimized. Another important application is noise reduction in noisy images. Noise is referred to the distortion of the analog signal while traveling across the interface cable. Thus, pixels are generated with values that exhibit the effect of noise; 


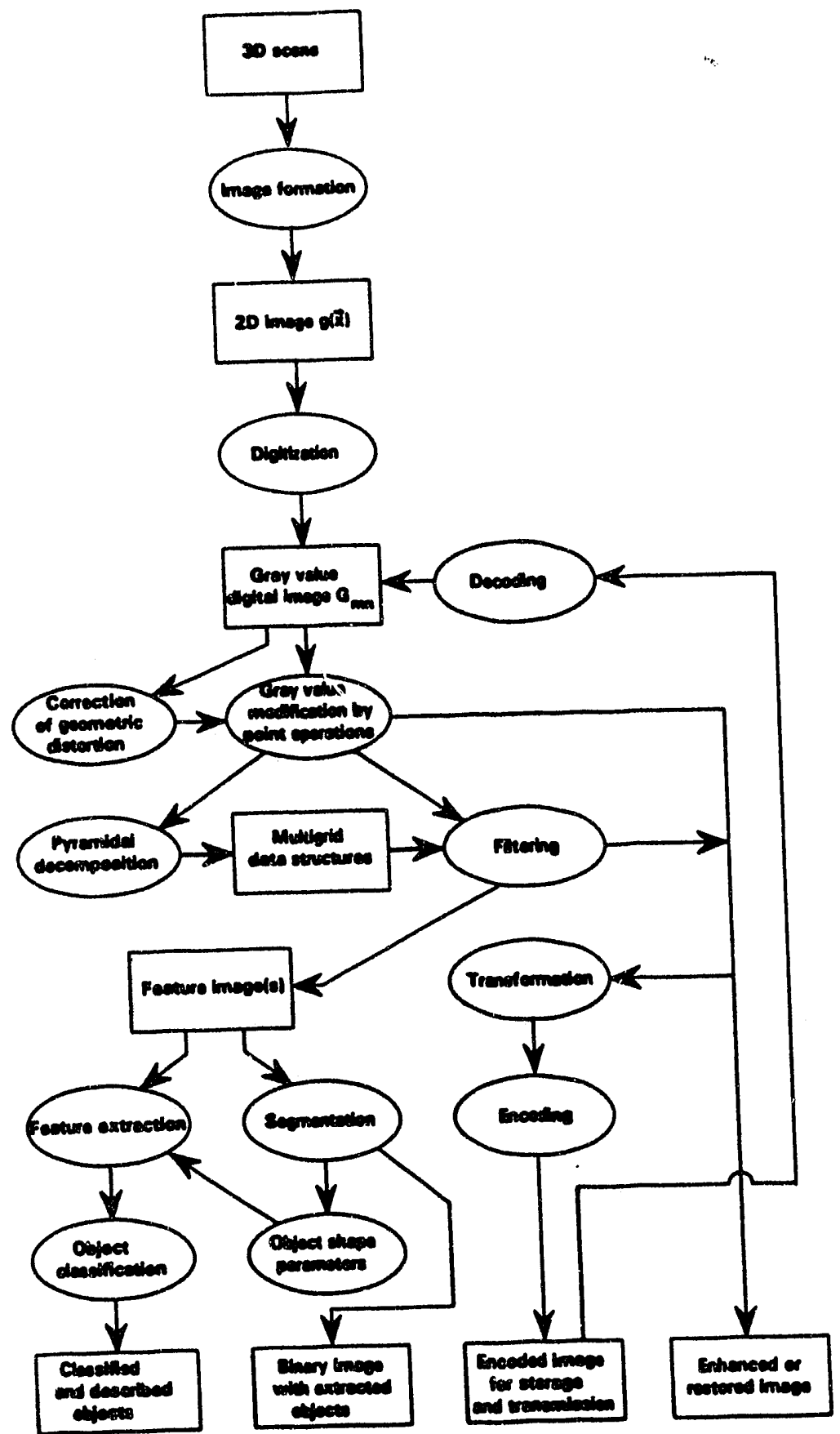

Figure 11. A Hierarchy of Digital Image Processing Operations 
pixel values change as impacted by noise.

The next phases depend on the aim of image processing. Sometimes only removing sensor-related errors from the image or enhancing the contrast is required. Effective transmission and storage of images necessitates a further step. In order to cope with the enormous amount of image data, the images must be stored and transmitted in the tightest possible code. Some types of images may allow errors in the coding process, other types may not.

A whole chain of processing steps is necessary to analyze and identify objects. First, adequate filtering procedures must be applied in order to distinguish the objects of interest from other objects and background. Then the object has to be separated from the background (segmentation). This process leads to a binary image. Now that the exact geometrical shape of the object is known, further information can be extracted as the mean gray value, the area, perimeter, and other parameters for the form of the object. These parameters can be used to classify objects (classification).

\section{2 Lenses and Light Values}

Image acquisition is the first step of digital image processing and is often not properly taken into account. However, quantative analysis of any images requires a good understanding of the image formation process. A necessary step for an object in the three-dimensional world to become a digital image in the memory of a computer is to become visible. An object becomes visible by the interaction with light or, more 
generally, electromagnetic radiation. The four basic types of interaction are reflection, refraction, absorption, and scattering. These effects depend on the optical properties of the material from which the object is made and on its surface structure. The light collected by a camera system is determined by these optical properties as well as by the illumination, i.e., position and nature of the light or, more generally, radiation sources.

To form an image, an optical arrangement of glass at the front of a camera (lens) is mounted. The lens brings the image of the scene into sharp focus on the CID imager. It's optical characteristics determine field of view (how much of the total scene in front of the camera appears in the monitor picture), depth-of-field (range of distances in front of the camera over which objects can be brought into focus), and the amount of light reflected by the scene that falls on the imager. Lenses generally reduce total light reaching the imager, an important consideration in low-light applications where the luminance of the object may be enough to generate a useable video signal based on the camera's sensitivity, but inadequate after it passes through the optics and is reduced to a value that does not yield a useable signal.

Standard lenses feature both focus and aperture (iris) controls and may be used over a wide range of applications. There is a limit to the minimum lens-to-object distance for these lenses. For closer distances, extension tubes are used on these lenses, allowing the lens-to-array distance to be increased, thus further reducing the minimum object distance.

The lens works by refraction or the bending of light beams. Refraction occurs 
because light travels slower in the denser glass medium. The amount by which the ray is bent is determined by the index of refraction of the optical glass and the angle of incidence at which the light strikes the glass. The distance between the prime focus point (center of the sensor) and the center of the lens is called the focal length.

Camera specifications often include both the scene illumination at which the imager saturates and the minimum illumination that will yield a useable video signal. Although camera operation deals with the amount of light reflected by the subject that actually falls upon the imager, it has become common practice to work with the light falling on the object, that is, the illumination of the scene.

Measurement of illumination starts with the intensity of the light sources and its distance from that source. A foot-candle is the light falling on a surface one foot from a 1-candlepower source. The unit for illumination used in international standards is the lux. A rough conversion from foot-candles to lux can be made by multiplying footcandles by 10 . The lux setting of a typical CID camera is $0.7 \mathrm{ft}$ candle (or 7 lux). 


\section{CHAPTER V}

\section{EXPERIMENTAL DESCRIPTION}

This chapter presents a description of the experimental setup employed in this research project. The procedures applied in the experiments of: miniature isolated candles, two-candle arrays, isolated char/coal particles and two-particle char/coal arrays are discussed here as well.

\section{V.1 Experimental Setup}

The experimental facility which has been developed for this research project centers around two major components: a flat flame burner and a digital image processing system. Description of each component is given here.

\section{V.1.1 Flat Flame Bumer}

The burner used in this research project is a flat flame burner manufactured by McKenna Products. It is a pre-mixed burner used for studies on combustion, flame propagation and energy related research. It consists of a water cooled sintered porous disk made of 316 Stainless-Steel surrounded by a sintered porous shroud ring for the introduction of a shielding gas (nitrogen) flows to suppress aerodynamic instabilities, both enclosed in a precision machined housing assembly with connections for water cooling, research gas, and shroud gas. A schematic of this burner is presented in Figure 12. The burner is of cylindrical construction with a $6 \mathrm{~cm}$ diameter flame area. The flame 

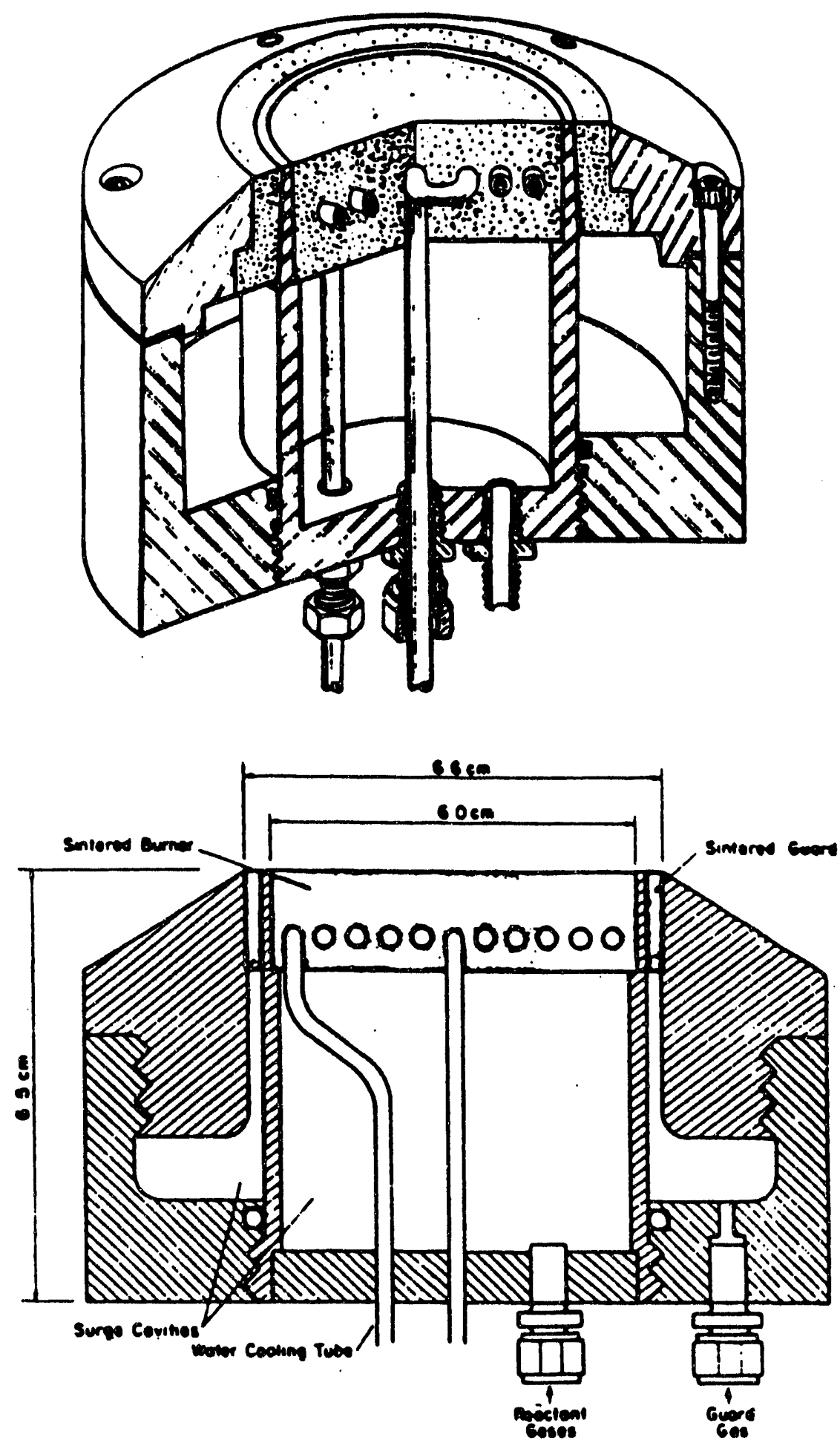

Figure 12. Schematic of Flat Flame Burner 
produced is stabilized above this $6 \mathrm{~cm}$ diameter, sintered, Stainless-Steel plug with cooling coils embedded in it. Water is used as the active cooling medium to facilitate rapid flame stabilization and to produce a temperature gradient opposing the radial diffusion of heat due to the flame. Reactant gases consisting of premixed air and fuel are introduced to the lower part of the burner. Any surges which exist in the reactant stream at this point are damped out in the cavity located below the sintered plug. The flames produced are shielded from the entrainment effects by passing a concentric inert gas $\left(\mathrm{N}_{2}\right)$ around the flame holder through a stainless-steel porous plug of $6.6 \mathrm{~cm}$ diameter. The inert gas is also introduced to the bottom of the burner into a cavity so that any surges present are damped out.

Conventional fuel (methane) and inert shroud gas (nitrogen) are supplied from standard high pressure cylinders (Figure 13). Rotameters (0-5 scfh and 0-200 sch respectively) with high accuracy needle valves are used for flow metering. Methane and air are premixed in the mixing chamber and the premixed gases passes through a rotameter unit (0-200 scfh), a flash arrestor device and finally through the cavities into the burners. The flash arrestor prevents any back fire from propagating back to the fuel cylinder. The premixed reactant stream is subsequently transported in a polyethylene tubing to the burner. Water, serving as the cooling medium, is transported to the burner in a polyethylene tubing and metered by a rotameter unit (0-200 scfh). The burner set up is mounted on a movable platform which enables adjustments of residence time and provides more flexibility for various measurements and operations. 


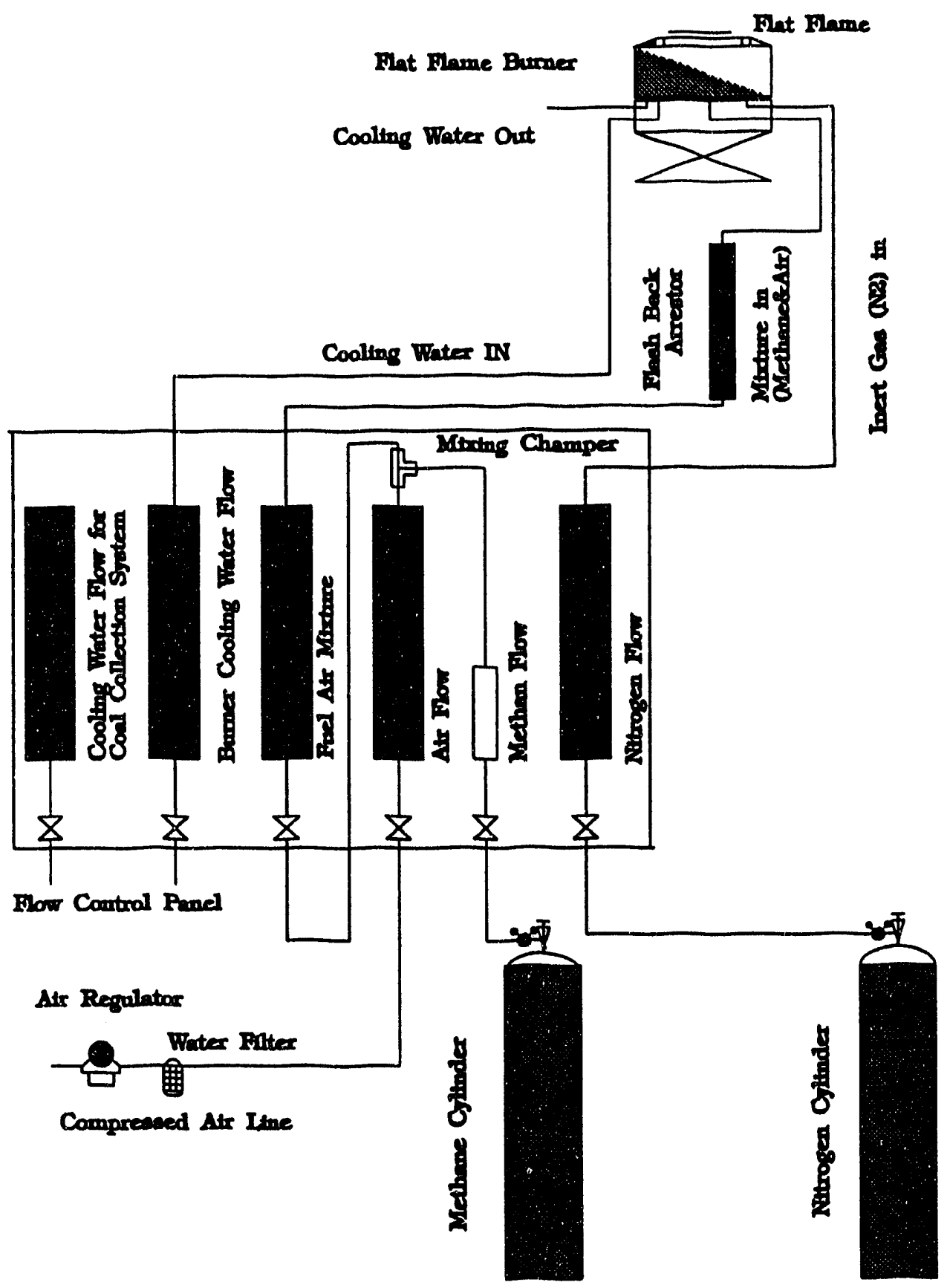

Figure 13. Schematic of Flat Flame Burner Facility 
Fine quartz rods (obtained from the glass blower shop, Chemistry Department), $0.35 \mathrm{~mm}$ diameter and $15 \mathrm{~mm}$ long, are utilized to hold char/coal isolated samples (glued to the tip of the quartz rod) on top of the flat flame burner. The rod is positioned at distance of $2.5 \mathrm{~cm}$ from the top of the burner. A gas temperature of $754^{\circ} \mathrm{C}(1027$ K) was measured at that location. Quartz was selected since it maintains a low thermal conciuctivity coefficient, $2.06 \mathrm{~W} / \mathrm{m}^{\circ} \mathrm{C}$ at $1200^{\circ} \mathrm{C}$, thus minimizes the conductive heat loss from the particle through the rod. Also, it is worth noting that the melting point for quartz is $1400^{\circ} \mathrm{C}$. Additionally, selecting the appropriate glue was equally important, since the glue was exposed to the same experimental conditions experienced by the char/coal particles. The applied glue is a single component ceramic adhesive based on Mica platlets and proprietary ceramic binders with a thermal conductivity of 0.87 $\mathrm{W} / \mathrm{m}^{\circ} \mathrm{C}$ and a service temperature that ranges from $150^{\circ} \mathrm{C}$ to $1260^{\circ} \mathrm{C}$. The melting temperatures and thermal conductivity coefficients of the quartz rod and the ceramic glue are significant properties that may affect the behavior of char/coal particles during the combustion process, especially since the temperature of the flat flame burner operational regimes ranges from $430{ }^{\circ} \mathrm{C}$ to $1100^{\circ} \mathrm{C}$ and the combustion experiments of char/coal particles were conducted at a temperature of $754^{\circ} \mathrm{C}$.

In addition to the basic facilities, the techniques of digital image processing are employed here.

\section{V.1.2 Digital Image Processing System}

In this section, a detailed description of the employed image processing system 
is given, inclusive of describing features, structure, and the operation of it's five major components, but prior to this, a brief description of the various operations involved in the image processing teclinique will be presented (Figure 14).

Typically, image processing applications involve performing various operations in order to extract statistical data and useful information of any desired process. The interaction between matter and radiation is the basis for all imaging. As light falls on the object, it becomes visible allowing the image acquisition device (a CID camera in this case) to sense the features of the object and record it. In addition to better illumination of the object, a fraction of the incident light gets reflected. This reflection, then, is picked up by the camera through the camera lens. Unlike low light applications where a direct (extra) light source is needed, the current application was fulfilled using natural light illumination. The next operation involves image acquisition. This is done using a 512 X 512 CID camera in which the camera imagery array, photodetector elements (located in the remote head), absorbs photons and translates these photon values to voltage levels forming an analog signal, which is stored in the camera or transported to another device. In the present application, the analog signals are transported via the control box to the frame grabber imaging board. The control box serves to enable the functions of the camera (more description is given later). In the board, the analog signal is converted to a digital signal, through the analog to digital converter $(A / D)$. The board is capable of storing and processing these signals with high accuracy. Various mathematical functions are supported with this board which enable 


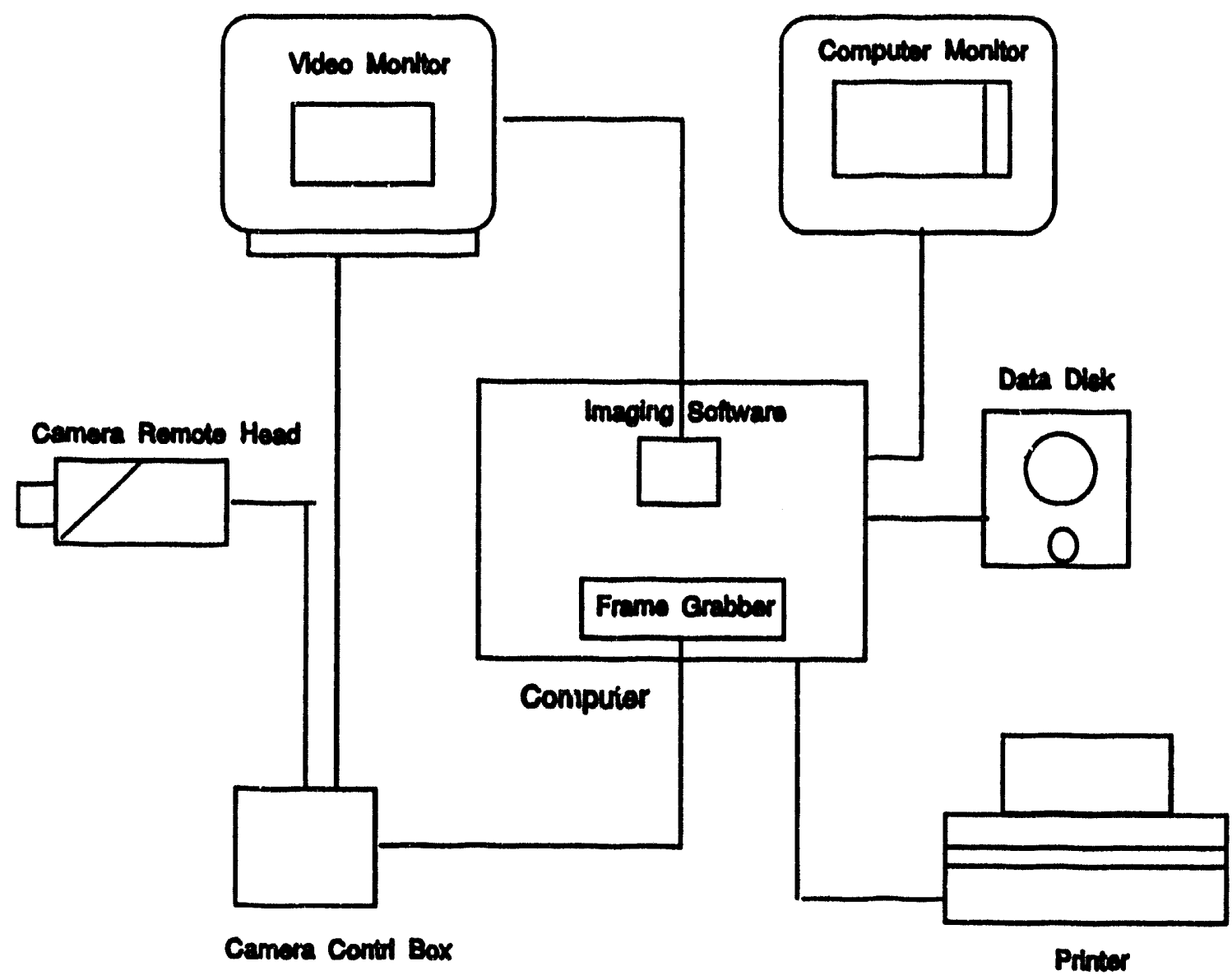

Figure 14. Image Processing Block Diagram 
the application of the image processing techniques. But before the image processing techniques can be applied, the digital signal needs to be transferred to numerical values (digitization) that the computer will understand. This is also done in the frame grabber. On the other hand, to access the functionality of the imaging board, an imaging software is utilized. Both, the imaging frame grabber and the supporting software are housed in a PC computer. The PC enables the interface between the imaging board and the software. A certain group of pixels form an image. The image can be displayed live on a video monitor, analyzed, modified, saved in the computer hard drive or on floppy diskettes, and eventually printed out. With this, the process of image processing is completed. It is interesting to note that while the camera is in the capturing mode, the images can be displayed live on the monitor for better adjustment and calibration of the camera's lens and the light source luminacity.

\section{Y.1.2.1 Camera}

The employed camera is a CID2250D $512 \times 512$ solid-state monochrome video camera, featuring a progressively scanned CID array: The sensor is packaged in a compact remote head, which is connected to a control unit (box) via a single cable (Figure 15). It is capable of fulfilling many automation and signal processing needs.

The key to this camera is the CID, a solid state sensor. With 512 rows $\times 512$ columns, the CID2250D is one of the highest resolution cameras available. The CID 35 solid state sensor, having square pixels (15 $\mu \mathrm{m} \times 15 \mu \mathrm{m})$ and $11 \mathrm{~mm}\left(2 / 3^{\prime \prime}\right.$ Diagonal) image array size, eases mathematical analysis of the video information. 


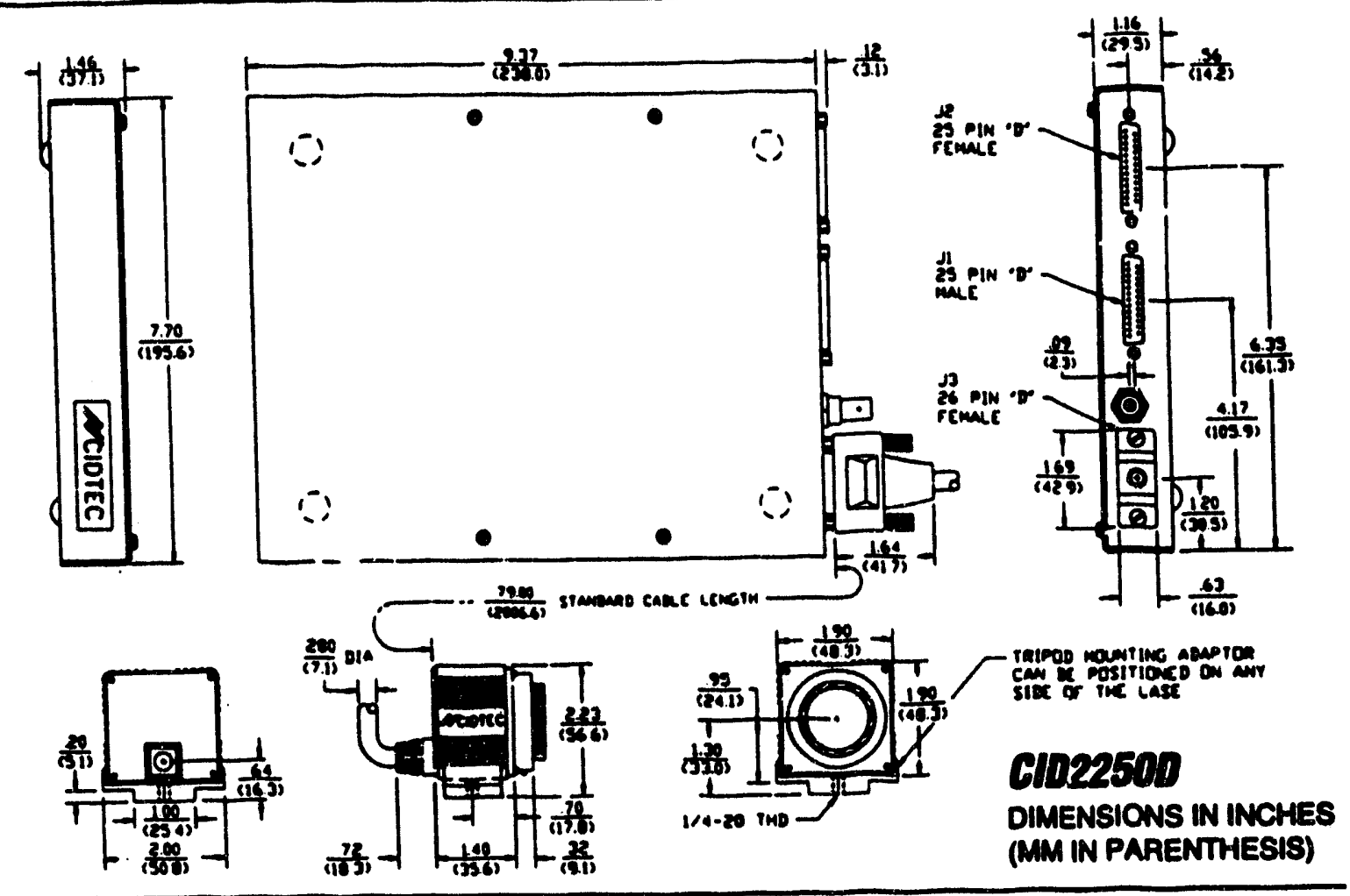

Figure 15. Schematic of the CID2250D Camera 
The timing of the CID2250D is realized with sophisticated Logic Cell Array (LCA) technology. The element rate clock (ERC), at $10 \mathrm{MHz}$ (100 ns), is suitable for fast video processing. The ERC is a built in chip that enables scanning one element (pixel) at a rate of $10 \mathrm{MHZ}$. The CID is read out in the sequential, progressive scan format. This negates the time typically necessary for interlaced to non-interlaced conversion; in the case of interlaced sampling more time is consumed to scan two fields. With a fast analog to digital converter the information can be processed during real time without the use of a frame buffer. CID2250D scans an image from left to right and from top to bottom. The scanning starts with the top left comer of the image and proceeds to the first horizontal line, and then the second horizontal line until the whole image is scanned in this way. Scan frequencies are as follows: i.) element duration is 100 ns (10 MHZ); the camera scans one pixel att that rate, ii.) line rate is $15.748 \mathrm{KHZ}(63.5 \mu \mathrm{sec})$; the camera scans each line, which includes 512 active pixels (635: blanking 123 pixels), at that rate, iii.) frame rate is $29.6 \mathrm{~Hz}(512 \times 63.5=33.8 \mathrm{~ms})$; the camera scans one frame, which includes 512 active lines (532: blanking 20 lines), at that rate. Thus, the output of the camera is 30 frames/sec.

In order to bring the image of the scene into sharp focus on the CID imager, a $25 \mathrm{~mm} \mathrm{X} 1.4$ (focal length $\times$ F-stop) lens is used. The lens features a " $C^{\text {" type mount }}$ which uses a 32 pitch threaded cylinder 1" in diameter. In establishing correct focusing of the image onto the sensor, the back-focus adjustment is employed. It is simply a threaded ring screwed into the lens mount (the lens screws into the ring), allowing the 
user to screw the lens mounting in or out, adjusting the focal length until the image throu ${ }_{\bullet} h$ the lens is optimally focussed onte the imager. Since this lens has a limit to the minimum lens-to-object distance, a $7 \mathrm{~mm}$ extension tube is added to the it enabling its focusing scale to have a range of minimum distance from 5.9" to 6.7" (Appendix A).

\section{V.1,2,1.1 Features}

The unique features of the CID2250 camera include frame reset, dump and rapid scan. Frame reset (FR) is a popular feature. When an object comes into view on an automated assembly line, the CID can be synchronously reset to zero and a new picture taken. The dump (DUMP) mode, frequently combined with frame reset, will clear the CID of all previous signal charge. Using the CID2250D in the frame reset mode greatly increases the system throughput.

The rapid scan feature (RSCN) is a window extraction feature. It is used to skip over useless video information. For example, if a single coal particle is to be captured, this function enables the user to zoom in at the particle location and scan only few lines covering the view of the particle. This reduces the scanned lines in a frame drastically, thus more frames can be scanned in $30 \mathrm{msec}$. This feature reduces data volume and improves system throughput by shortening the camera's frame rate. In practice, a number of windows may be accommodated; they may be changed, deleted or added on the frame to frame basis if desired. The rapid scan feature can be used in conjunction with the frame reset command.

A feature common to all CID cameras is the inject inhibit mode $(\mathrm{I} / \mathrm{T})$. The inject 
inhibit mode is used to extend video integration time for greater sensitivity under a low light level condition and for snapshot capture of transient events.

Interfacing the CID2250D with a control device, interface control module (ICM), will access other unique features included in the camera (not acquired). The most important feature is increasing the frame rate output to more than 30 frames $/ \mathrm{sec}$; frame rate output can be increased up to 700 frames/sec.

\section{V.1,2.2 Image Processing Board}

\section{V.1.2.2.1 Features}

The image processing board (4MEG VIDEO MODEL10 Frame Grabber) is an image acquisition and processing device (Figure 16). It offers unparalleled flexibility in video acquisition and display. Analog video signals can be digitized at up to 20 million samples per second. Alternatively, the board can accept direct 8-bit digital data. The pixel clock can be derived from an external source or generated internally. An adaptable video timing generator allows easy integration with image sources such as the highresolution CCD cameras. Programmable video resolution is provided by a unique horizontal control memory (HCM) that allows pixel-by-pixel control over image memory transfer during acquisition and display. Integer zoom and subsampling are supported also.

The frame grabber facilitates acceleration of image processing functions with 10 million instruction per second Texas Instruments TMS320C25 Digital Signal Processor. In addition to performing math-intensive image processing functions at high speed, the 


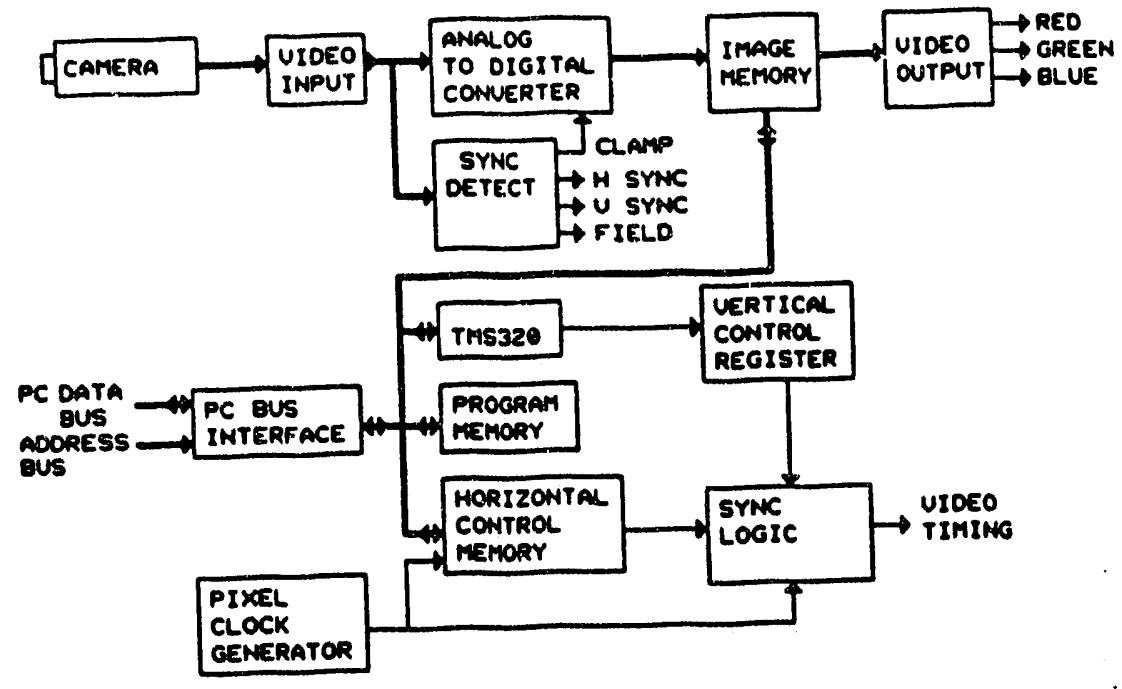

Figure 16. Architecture of Image Processing Board 
TMS320C25 can function much like a general-purpose microprocessor. This facilitates the implementation of custom image processing and inspection algorithms.

Pixel data is stored in image memory as sequential 8-bit values. The total image memory available is 4 Megabytes. Using the $\mathrm{HCM}$, this memory can be organized as one large image, or many smaller images. The largest image contains 512 X 512 pixels, thus a maximum of 16 frames can be stored; each frame occupies 1/4 Megabyte. On the other hand, smaller images such as $248 \times 248$ pixels images can be stored which leads to increasing the maximum number of images that can be stored in the overall memory. Portions of the memory can also be used to store intermediate results, menus, or overlays. A bit - plane write protect feature allows a text and overlays to be written over image data. The frame grabber occupies a single slot in a PC/XT/AT or compatible computer

During image acquisition, the display is driven with live video. Image processing can be performed during video blanking intervals to maintain an uninterrupted display. For display, three look-up tables drive red, green and blue Digital-to-Analog Converters (DACs), providing pseudo-color output. Usually video synchronization is on the green output.

\section{V.1.2.3 4MIP Image Processing Software}

The 4MIP is an interactive, menu driven program which operates the 4MEG VIDEO frame grabber to perform image acquisition, display, and processing. 


\section{V.1.2.3.1 Requirements}

i.) IBM PC/XT/AT or $100 \%$ compatible with $640 \mathrm{Kbyte}$ of memory, with monochrome or graphics display adapter and monitor. Most features of 4MIP can be operated with 512 Kbyte of memory.

ii.) 4MEG VIDEO frame grabber, with either 4 Mbyte or 1 Mbyte memory, with 2K, $8 \mathrm{~K}$, or $32 \mathrm{~K}$ HCM, with or without RGB option.

\section{V.1.2.3.2 Features}

This section provides an overview of the features available in the 4MIP program.

The Video Formats allows the current video format to be examined and changed. Standard formats such as RS-170 can be selected along with the ability to custom design a video format. Simple options allow digitizing from interlaced and noninterlaced cameras, and digitizing an image into memory with left/right or top/bottom rotation. Briefly, in interlaced sampling, the horizontal lines which constitute one frame of displayable video are split into two fields, each field is composed of alternate lines. For example, in RS-170 the number of vertical lines, and thus the maximum vertical dimension, is $\mathbf{4 8 0}$ split into two fields of 240 lines each (interlaced sampling). Upon displaying, both fields are displayed to form the image. On the other hand, for a non-interlaced sampling such as CID2250 the number of the maximum vertical dimension is 480 in one field. This uses less memory and insensitive to shaking while capturing images. Some special modes of operation, such as CID2250 and other special cameras, incorporate predefined custom formats and are set in Special 
Operations \& Modes.

The Video Resolution allows the current video resolution and position to be examined and changed. In the horizontal (vertical) direction, the number of sampled pixels (sampled lines), the spacing between sampled pixels (lines), and the left/right (top/bottom) position can be adjusted. The number of image buffers can be increased decreasing the number of samples used per image. For an interlaced format (such as RS170), distinguished field or repeat field modes can be selected.

The Video Digitize/Display allows selection of the digitize (image capture) mode or the display mode and whether each uses genlock or master mode. The image buffer to be displayed or digitized can be selected. A submenu provides for split screen digitize and display modes; digitizing (capturing) a new image in $1 / 2$ or $1 / 4$ of the screen while the remainder of the current image is unchanged. The split screen boundaries are by default image halves, but can be altered in Video Resolution.

The Motion Sequence Capture/Display allows a sequence of images to be digitized (captured) and displayed at a fixed intervals of time. The digitize and display rate can be as frequent as one field time (1/60 second for RS-170). The maximum length of the sequence depends on the number of image buffers available, and thus on the Video Resolution selected. A sequence can be digitized (captured) into a screen half, allowing multiple sequences to be captured and corresponding frames to be compared. The sequence of identical images (camera's field of view stationary) can be captured and averaged pixel by pixel; thereby enhancing image quality without smearing. 
The Special Operations and Modes contains various special operations, and the setting of predefined custom modes. One such operation is a toggle operation which alternately digitizes and displays a pair of image buffers at a frame or field rates, allowing interactive registration of a new image to match an old image.

The Contrast and Lockup Tables allows the RGB lockup tables to be examined and modified. Multiple table sets are supported, allowing rapid switching among several choices. RGB table sets can be saved to and loaded from files.

The Image Zoom \& Pan allows an image area (panned) of the currently displayed image to be expanded to full screen (zoom in). Zoom factors of 2, 4, 6, 8, etc. are supported; some video resolutions also allow odd zoom ratios. Pan can be operated whether zoom out or in, as can selection of the current image buffer.

The Pixel Peek, Poke \& Plot allows examination and alteration of specific pixels. A cursor can be positioned and $X-Y$ coordinates displayed. Individual pixels can be altered, and values for the $17 \times 17$ box of pixels around the cursor displayed. Image buffers can be selected while the cursor is being positioned, and/or pixel values displayed. Lines or columns of pixel values can be graphed.

The Processing Modes selects modes under which Image Test Patterns \& Sequences, Image processing, Image Printing, and Image File Load/Store operate. One mode selects whether the area of operation is the full image in the current image buffer, or is to be selected as some rectangular image window when any operation is later requested. If the latter is enabled, an image widow can be selected by $X-Y$ 
coordinates, screen quadrants, or by interactive drawing of a box with cursor and ARROW keys (or mouse). A second mode selects whether the operation will execute with image display on, or image display off; the later is faster, but the former more interesting.

The Image Test Patterns \& Sequences allows the generation and display of various test patterns into the full image buffer, or an image window. these patterns are useful for image monitor adjustment (intensity, contrast, linearity) or for judgement of monitor quality (line Pairs).

The Image Processing contains several submenus. All of the image processing operations can be performed on either the full image or an image widow, as set by the Processing Modes.

The Image Processing - Histogram Displays allow calculation and display of histograms of either the full image or any image window. The histogram can be displayed as a listing, vertical chart with interactive paging, or as a horizontal graph with interactive viewing analysis. Percentile rank, mean, variance, and similar descriptive statistics are also available.

The Image Processing - Simple Pixel Operations include setting pixels to a constant, bit AND and XOR, grey level complement, contrast enhancement based on the low and high bounds expressed as pixel values or expressed as histogram percentiles, scaling of pixel values by a multiplication constant, thresholding, addition of random noise (dither), and mapping pixels through a lockup table. 
The Image Processing - Image Copy, Resize, Rotate provides operations to copy an image without modifications as well as resize, rotate, shift, and flip images while copying; operating upon full images in different image buffers, or image windows in the same or different buffers.

The Image Processing - Two Image Operations includes operations to perform image addition and subtraction with various options for underflow or overflow, exclusive or, logical and, product, ratio, average, and arbitrary mathematical functions of corresponding pixels of two images. Also available is comparison of two images noting (in black pixels) areas which are identical, and contrast modifying a new image to match (to the first 2 moments) the characteristics of an old image; operating upon full images in different image buffers, or image windows in the same or different buffers.

The Image Processing - 3x3 Operations includes low pass filtering, low smear low pass filtering, sharpening, three median filters, rank filters, and Kirch, Sobel and Roberts $(2 \times 2)$ edge detectors and edge thinners.

The Image Processing - NxN Operations supports convolution with arbitrary coefficients on $3 \times 3$ through $25 \times 25$ neighborhoods. Submenues provide for setting of the coefficients. Other operations include dynamic threshold and inverse contrast ratio mapping (statistical scaling), each based upon a neighborhood of up to size $25 \times 25$.

The Image Processing - Morphological Operations includes erosion, dilation, open, close, boundary detection, hit-miss mask comparison and thinning upon binary images. Various sizes and shapes of morphological structure elements can be chosen. 
The Image Processing - Transform (FFT) Operations allow computation of Discrete Fourier Transforms (Fast Fourier Transforms) of an image, log magnitude display of the frequency domain, graph magnitude or phase, filtering in the frequency domain, and inverse transforms.

The Image Processing - Image Interlace Modifications provides operations to de-flicker interlaced images and for conversions between the two image line organizations commonly used for interlaced images.

The Image Processing - Moments \& Center of Mass provides quick calculations of mass and center of mass, and provides more detailed calculations of up to 3rd order moments and related descriptive statistics.

The Image Processing - Miscellaneous Operations includes image resample/binning with optional shrinking (average each rectangular bin of pixels, replace pixels with average, or set one pixel of new image with average), intensity normalization, halftoning, and averaging of corresponding pixels of multiple images.

The Image Processing - TMS320 Resident Operations provide image operations run on the TMS320 processor on the 4MEG VIDEO; the image operations described above are computed on the PC's processor. A $3 \times 3$ convolution is available, with predefined coefficients for low pass, sharpen, edge detect (Laplacian) as well as allowing user defined coefficients. Morphological operations available are erode, dilate, and boundary detection. Also available are image sequence average and image sequence differences. 
The Image Measurements allows calibrating image rulers in arbitrary units, then moving and rotating the rulers to measure image object distances and angels. A second set of operations allows placing markers upon image objects, in one or multiple buffers, then graphing the relative distances between markers.

The Paint, Draw \& Text Overlay provides for interactive painting of patterns onto the image using various size paintbrushes, and allows interactive drawing of line, ellipse, arrow, Bezier polynomial, and free hand curves. Image areas, bounded by drawn curves, can be filled with patterns. Textual legends can be written onto the image; the legends can originate from a file or from the keyboard.

The Image Printing allows the printing of the full image, or an image window, to various printers.

\section{Y.1.2.4486 Personal Computer}

An essential component of the image processing system, a 486 personal computer, was employed and integrated with the rest of the system's components. It served as the housing unit of both the frame grabber and the image processing software. The functionality of the image processing board and software were enabled mainly by this computer. In order to meet the requirements for the operation of the frame grabber and the supporting software, the computer maintained many features and specifications. Some of these features are: intel 80486 C.P.U. rated and operating at $33 \mathrm{MHz}, 8 \mathrm{Mb}$ system R.A.M. expandable to $32 \mathrm{Mb}, 64 \mathrm{~kb}$ external CPU cache, Two floppy drives (3.5" $1.44 \mathrm{Mb}$ and 5.25" $1.2 \mathrm{Mb}$ ), fixed hard disk with $200 \mathrm{Mb}$ formatted (DOS) 
capacity, 14" diagonal monitor, 101-key enhanced keyboard, math coprocessor (INTEL), and two RS-232 serial and one centronics parallel ports.

\section{V.1.2.5 13" High Resolution Color Video Monitor.}

A high resolution color video monitor (Sony PVM-1342Q) is integrated with the image processing system to provide a tool for visual observation of both the digitizing and displaying processes. While digitizing, the combustion process is observed and the camera is focussed and it's position is adjusted, simply by visual observation of the monitor's displayed image. After image processing techniques are applied and the image is enhanced and modified, the new image is displayed on the monitor's screen allowing more adjustments to be done. Features of this monitor include:

i.) Color System: PAL, SECAM, NTSC, and NTSC 4.43, systems switched automatically.

ii.) Horizontal Resolution: more than 600 lines, video input; more than $900 \mathrm{H} \times 200 \mathrm{~V}$ pixels, RGB inputs.

iii.) Character display: 2000 characters (80 columns $\times 25$ lines).

iv.) Brightness: more than 47 foot-lamberts.

\section{V.1.2.6 Cable Connections}

The 4MEG frame grabber makes external connections with a female 25 pin DSubminiature connector. This connector is called the Analog $1 / O$ connector. 12 signals are available.

A set of three cables, IDB25-2250, necessary for digitization of the video signal 
by the 4 MEG VIDEO frame grabber. The cable set provides connection between $4 \mathrm{MEG}$ VIDEO and the camera control unit and a display monitor. The set consists of three cables - one connected to the 4MEG VIDEO (Analog I/O), one connected to J1 on the camera control unit, and one connected to $\mathrm{J} 2$ on the control unit. The cable connected to the Analog $\mathrm{I} / \mathrm{O}$ connector has the following connections:

i.) Video Input (VIN).

ii.) Pixel Clock Input (CLOCK_IN).

iii.) Composite Sync Output (COMP_OUT).

iv.) Red color Output (RED).

v.) Green Color Output (Green).

vi.) Blue color Output (Blue).

The $\mathrm{J} 1$ connector has the following connections:

i.) Analog Video Output (VIDEO OUT).

ii.) + 15VDC Input.

iii.) Ground Input.

iv.) Pin 3 connected to pin 25 .

v.) Pin 6 connected to pin 17 .

The connection between pin 3 and pin 25 is to power the control unit's output drivers. The connection between pin 6 and pin 17 is to add composite sync to the video output. The J2 connector has the following connection: Pixel Clock Output (CLK_OUT). Connections are to be implemented in the following manner: 
i.) Connect the Analog I/O to the 4MEG VIDEO.

ii.) Connect the $\mathrm{J} 1$ and the $\mathrm{J} 2$ cables to the camera control unit.

iii.) Connect the camera to the camera control unit.

iv.) Connect VIDEO OUTPUT from the J1 connector to the VIN of the analog I/O connector.

v.) Connect the CLK_OUT of the J2 connector to the CLOCK_IN of the Analog I/O connect or.

vi.) Connect the GREEN, RED, and BLUE signals of the 4MEG VIDEO to the respective inputs on the monitor.

\section{V.2 Experimental Procedure}

Preliminary experiments were conducted on miniature isolated candles as well as two-candle arrays experinents. In the experiment of an isolated candle, the candle was allowed to burn in a drift free environment to maintain burning rate stability. The mass burning rate of the candle was recorded and the experiment was stopped when the candle's mass loss became $40 \%$ of the original mass. In addition, images were captured for the burning process and were digitized and analyzed using the digital image processing system. Similar proc dure was followed in the experiment of two-candle arrays. Two similar candlesi were placed close to each other and were allowed to bum. Once $40 \%$ mass loss rate was reached by the candles, the experiment was stopped. Images of the burning prosess were also captured and analyzed. The same experiment 
was repeated while varying the distance between the centers of the two candles. The effect of the interparticle distance on the burning rate of the candles was determined.

The combustion of char/coal particles was enabled through employing the flat flame burner. The flat flame burner was lit, and the flame was allowed to stabilize on top forming a flat flame. Fuel and air mixtures feeding rates into the bumer were adjusted (1.5 and 28.5 scfh respectively) such that $12.4 \% \mathrm{O}_{2}$ concentration was obtained in the products of the fuel combustion. Single char/coal particles were glued to the tip of a fine quartz rod and were exposed suddenly to the hot environment provided by the flat flame burner. The sudden exposure of the char/coal particles is achieved by withdrawing a flat Aluminum plate positioned initially at a certain distance from the top of the burner. Ignition and combustion characteristics of these particles were monitored and images of the complete combustion process were captured through the digital image processing system. Images were captured for the whole combustion period. Captured images of the combustion process were then displayed on the monitor's screen, digitized, analyzed frame by frame, modified, and eventually printed out. Analyses and modifications were conducted by applying various functions and routines of the 4MIP imaging software to the captured frames on individuai basis. The same procedure was repeated for the combustion of two-particle arrays. Two particles of char/coal were placed adjacent to each other while allowing the interparticle distance among them to vary. To conduct our combustion analysis on the char/coal particles, density of coal was determined experimentally. A $100 \mathrm{mg}$ of coal was added to a $200 \mathrm{ml}$ beaker filled with 
water. Upon adding the coal, water was displaced outside the beaker. The displaced water volume was then measured. This volume was assumed to equal the volume of the coal added, thus the density of coal was calculated as maas/volume. The coal density was a bout $1300 \mathrm{~kg} / \mathrm{m}^{3}$ for the three types of coal. As for the char, a density of 1040 $\mathrm{kg} / \mathrm{m}^{3}$ was obtained.

Ignition and combustion characteristics were eventually determined for the isolated as well as two-particle arrays using the digital image processing techniques. 


\section{CHAPTER VI}

\section{RESULTS AND DISCUSSION}

The objective of this research study involves determining the ignition and combustion characteristics of isolated char/coal particles as well as two-particle arrays, employing digital image processing techniques.

Preliminary experiments were conducted on miniature candles in which the combustion behavior of an isolated candle was determined. Additionally, the effect of intercandle spacing ( $(/ a)$ on the candles (treated as particles) mass burning rate was explored. Subsequently, detailed experimental studies were conducted on char and coal particles.

This chapter presents the experimental results for: i.) isolated candles, ii.) twocandle arrays, iii.) isolated coal particles including high volatile, medium volatile, and low volatile coals, iv.) isolated char particles, v.) two-particle arrays including high and medium volatile coal and vi.) two-particle char arrays.

\section{VI.1 Isolated Candles}

In this experiment, an isolated candle was allowed to bum in a drift free environment. Table 1 summarizes the experimental data: mass, normalized mass with respect to initial mass and mass calculated from the regression analysis. As expected, the mass decreases as tine increases. The mass burning rate is computed from the 
Table 1. Isolated Candle Mass Variation with Time

\begin{tabular}{|c|c|c|c|}
\hline Time ( $\mathrm{g})$ & Mass (g) & Mass/Mass Initial & Mass-Regression \\
\hline 0 & 1.12 & 1.00 & 1.13 \\
\hline 15 & 1.1 & 0.98 & 1.11 \\
\hline 30 & 1.09 & 0.87 & 1.09 \\
\hline 45 & 1.07 & 0.86 & 1.07 \\
\hline 60 & 1.02 & 0.91 & 1.05 \\
\hline 75 & 1.03 & 0.92 & 1.02 \\
\hline 80 & 1.01 & 0.80 & 1.00 \\
\hline 105 & 0.98 & 0.87 & 0.98 \\
\hline 120 & 0.97 & 0.87 & 0.86 \\
\hline 135 & 0.94 & 0.84 & 0.94 \\
\hline 150 & 0.82 & 0.82 & 0.92 \\
\hline 165 & 0.92 & 0.82 & 0.80 \\
\hline 180 & 0.91 & 0.81 & 0.88 \\
\hline 195 & 0.84 & 0.75 & 0.86 \\
\hline 210 & 0.84 & 0.75 & 0.84 \\
\hline 225 & 0.82 & 0.73 & 0.81 \\
\hline 240 & 0.8 & 0.71 & 0.79 \\
\hline 255 & 0.77 & 0.69 & 0.77 \\
\hline 270 & 0.75 & 0.67 & 0.75 \\
\hline 285 & 0.75 & 0.67 & 0.73 \\
\hline 300 & 0.72 & 0.64 & 0.71 \\
\hline 315 & 0.64 & 0.57 & 0.69 \\
\hline
\end{tabular}

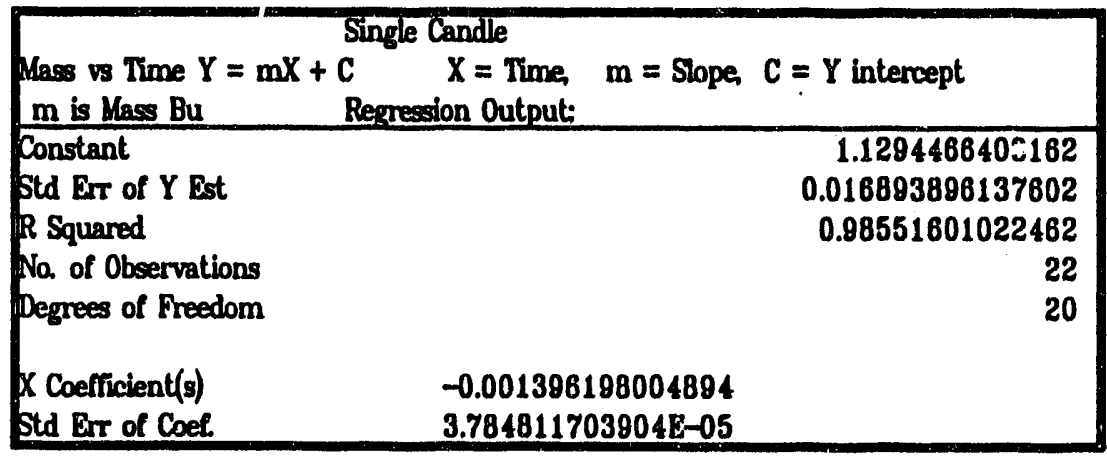


regression analysis. Figure 17 shows a graphical representation of these results. Also, images of the burning process were recorded and are shown in Figure 18. As the candle started to burn, a flame was formed around its wick of $0.98 \mathrm{~mm}$ thickness. As more time elapsed, the flame started to grow while consuming more and more oxygen from the surroundings. The burning process shows an interesting sequence in which the increase in the flame's size can be seen clearly (Figure 18a). Analyzing the burning process through the captured images, a black inner zone was observed inside the flame area. This is possibly due to the fact that once the candle is ignited, the fuel vapor starts consuming the local oxygens surrounding the wick. As the size of the formed flame (luminous zone) increases, there is increased feed back of heat to the candle resulting in more vapor production. Since local oxygens have been completely consumed, a fuel rich inner zone (black inner zone) is formed. The luminous outer zone is an indication of combustion of premixed fuel vapor and oxygens. It is interesting to note that the inner black zone followed the same course of the flame and grew larger and larger as more time elapsed. Figure $18 \mathrm{~b}$ indicates the boundaries of the flames, obtained with "Morphological Operations-Boundary Detection" function (Chapter V).

\section{VI.2 Two-Candle Arrays}

Experimental data of the mass burning rate of the two candles at each intercandle distance were obtained and tabulated in Appendix B. The tables include the total mass, mass per candle, normalized mass of the candles and the mass obtained from the 


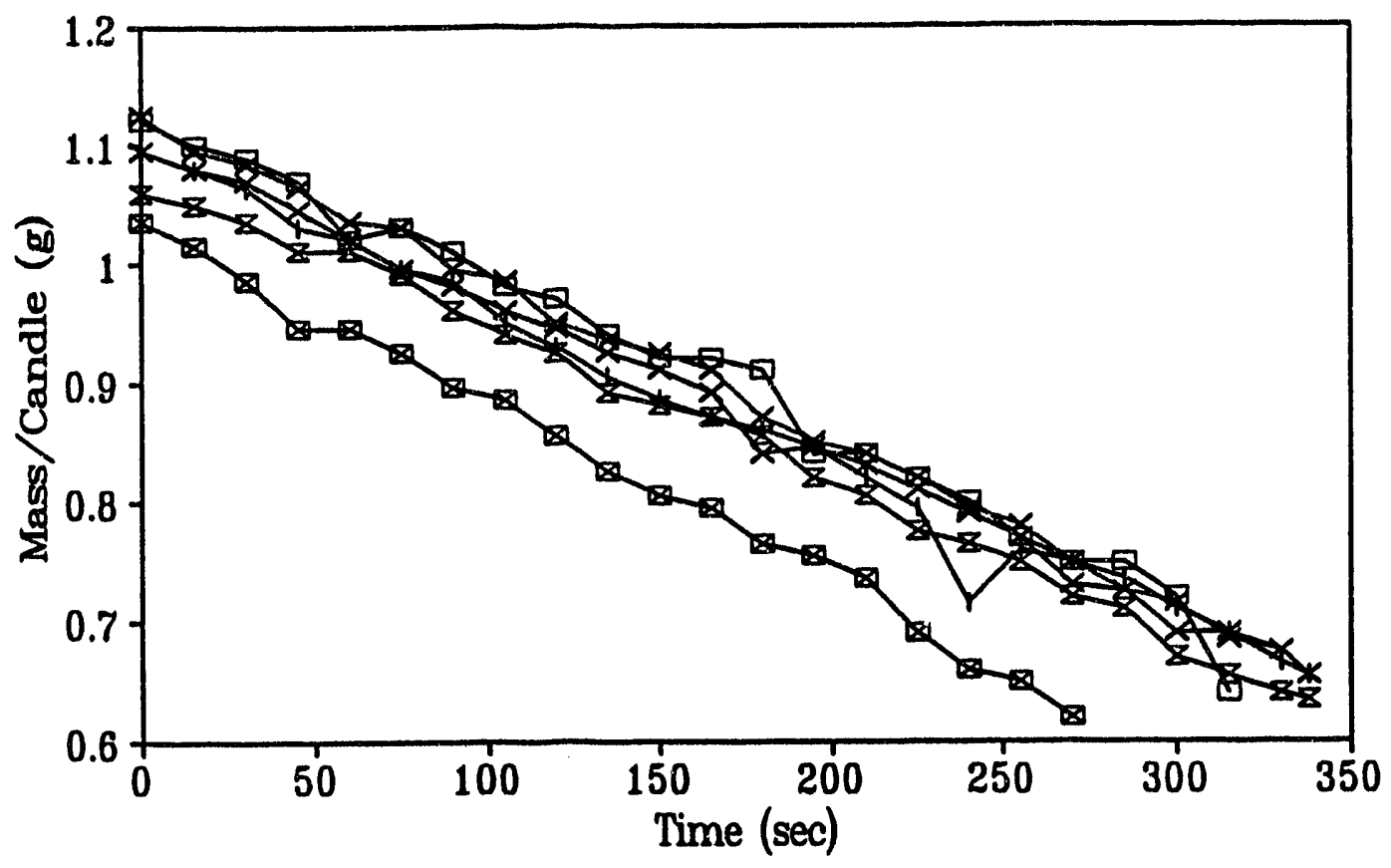

$\rightarrow$ Single Candle $\rightarrow$ Tro Candles $(6 \mathrm{~mm}) \rightarrow$ Tro Candles $(8 \mathrm{~mm})$

$\rightarrow$ Tro Candies $(12 \mathrm{~mm})+$ Two Candles $(15 \mathrm{~mm}) \rightarrow$ Two Candles $(17 \mathrm{~mm})$

Figure 17. Variation of Mass with Time for Isolated Candl: 

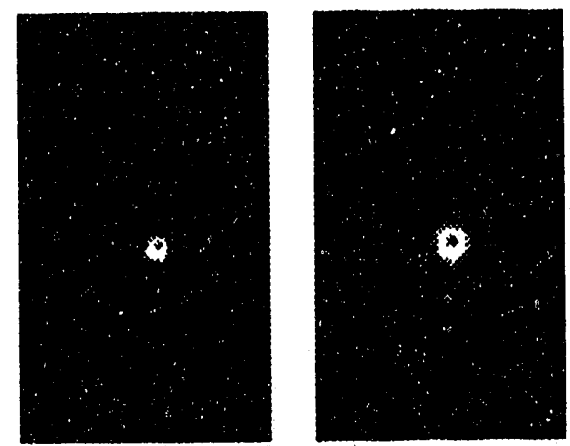

30

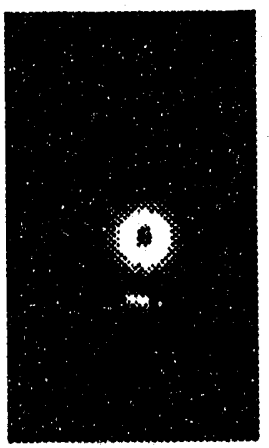

240

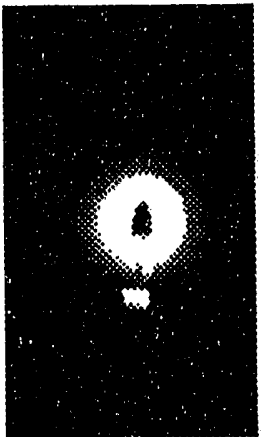

420
60

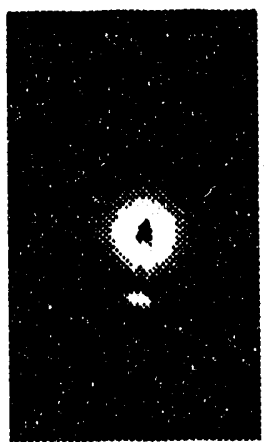

270

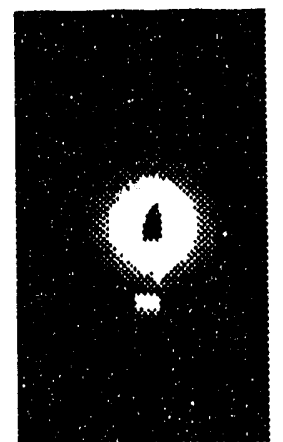

450

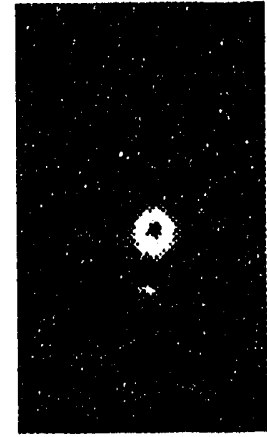

90

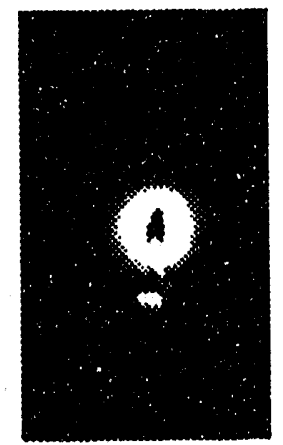

800

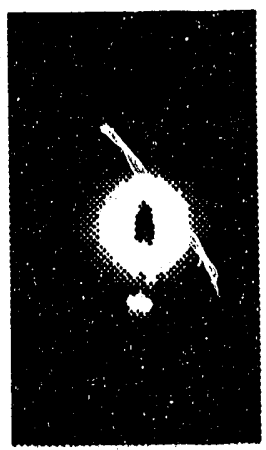

480

Figure 18a. The Burning Sequence for Isolated Candle (Time in $\mathrm{msec}$ ) 


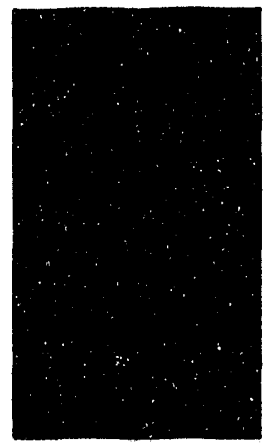

30

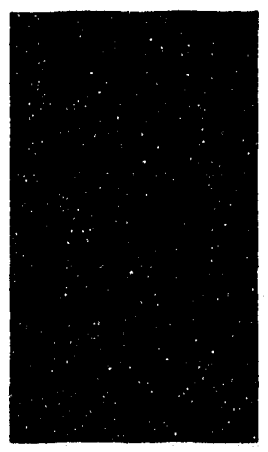

240

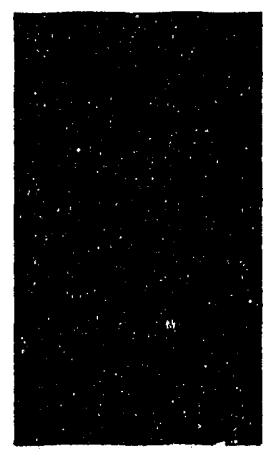

420

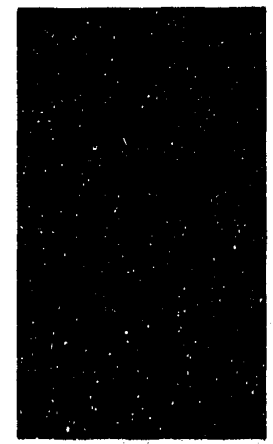

60

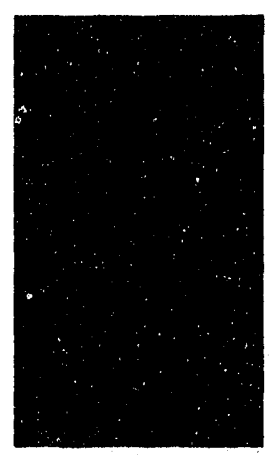

$\mathbf{2 7 0}$

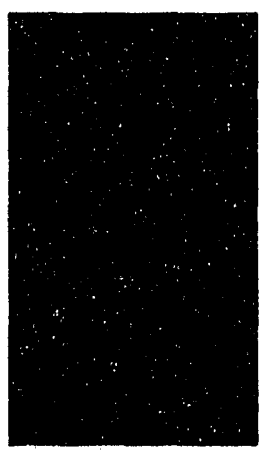

450

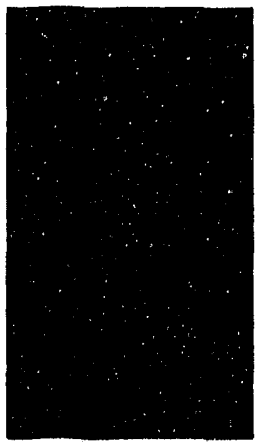

90

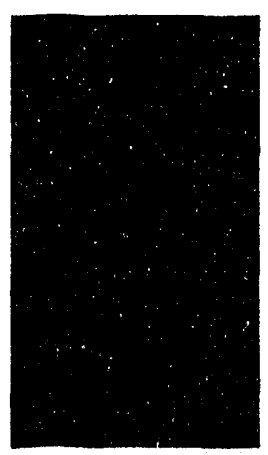

300

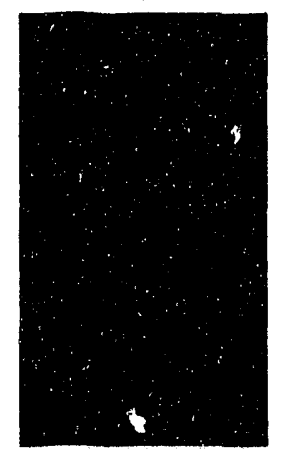

480

Figure 18b. Images of the Burning Sequence with Boundary Detection Function for Isolated Candle 
regression analysis. As the two candles were lit, flames grew larger with time due to the decrease in the local oxygen concentration. Eventually, at $t=t_{m}$ (flames merging time), the two flames contact each other. Once the flames merge, a group or a common flame is formed and hence the interactive effects become stronger. The mass versus time is recorded. Figure 19 plots normalized mass versus time. Regression analysis has been conducted for all the experiments and results are tabulated and plotted in Appendix B. The experimental data is used to determine the time for $40 \%$ mass loss of the candles. The results are graphically illustrated in Figure 20. As expected, upon decreasing the inter candle distance, the $40 \%$ burn time decreased due to increased free convection effect which increases the availability of oxygens for combustion. At closer inter candle spacing, the oxygen deficiency becomes dominant resulting in increased burn time. These results are similar to those obtained for a binary array of drops. This trend was observed in all two-candle arrays except in the case where the two candles were apart by $15 \mathrm{~mm}$. This can be attributed to a possible increase in the convection effect at this distance which lead to a higher burning rate and thus less elapsed time to burn.

While the two-candle array was burning, images of the burning process are captured, digitized and analyzed. Figure 21 shows the burning sequence for a two-candle array at $l=6 \mathrm{~mm}$. As candles were ignited, an incividual flame was formed around each candle's wick at $t=30$ msec. The flame sizes gradually increase with time due to more fuel vapor produced by the candles. Subsequently, the two flames grew individually until they merged together forming a common larger flame at $t=480$ 


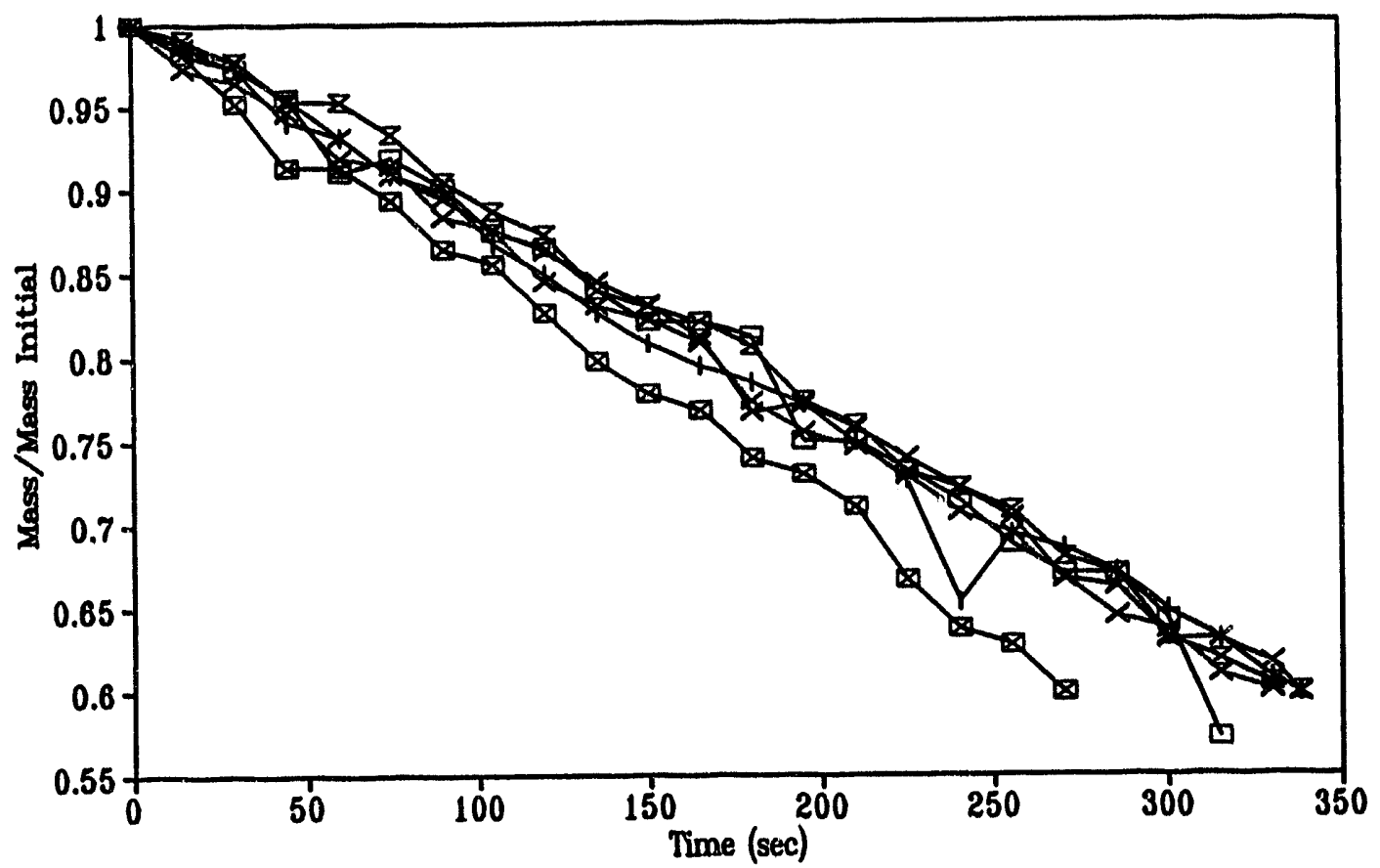

E-Single Candle $\rightarrow$ Two Candles $(6 \mathrm{~mm}) \rightarrow$ Two Candles $(9 \mathrm{~mm})$

-Tro Candles $(12 \mathrm{~mm})+$ Tro Candles $(15 \mathrm{~mm}) \rightarrow$ Tro Candles $(17 \mathrm{~mm})$

Figure 19. Variation of Normalized Candles Mass with Time for Candle Arrays 


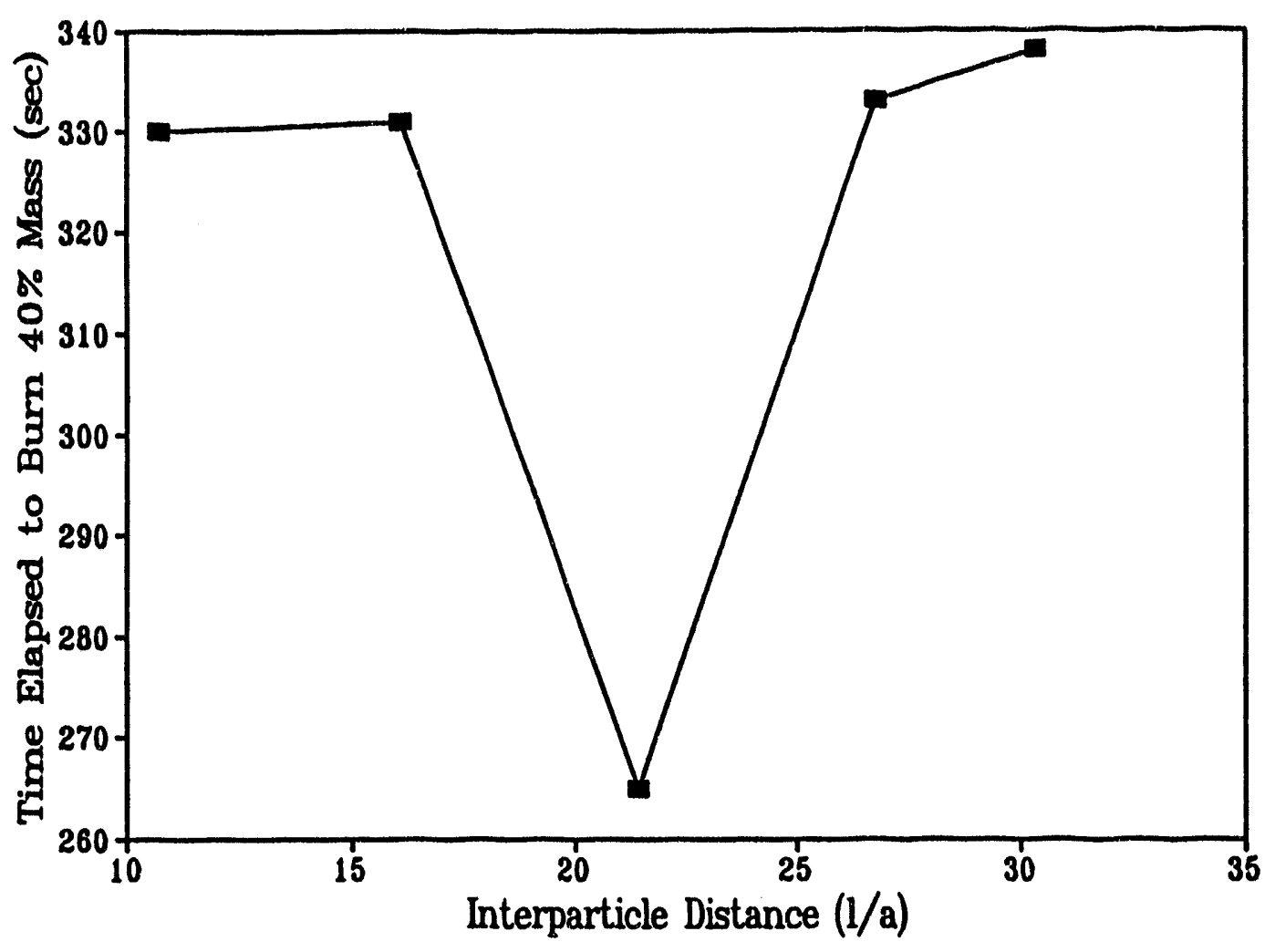

Figure 20. Variation of $40 \%$ Burn Time with Inter-Candle Spacing for Candle Arrays 


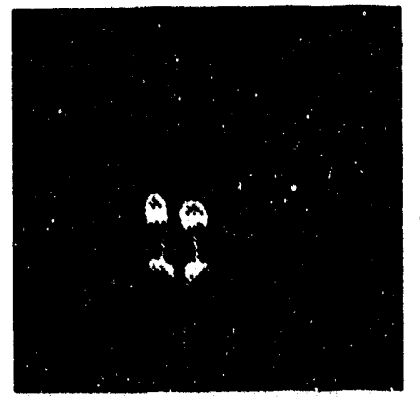

80

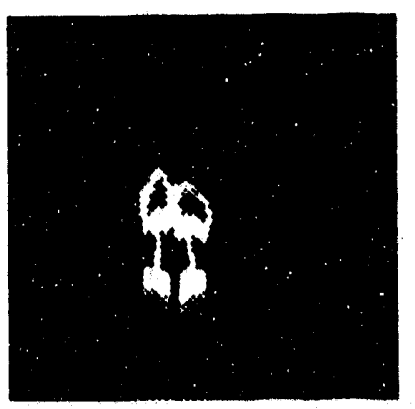

240

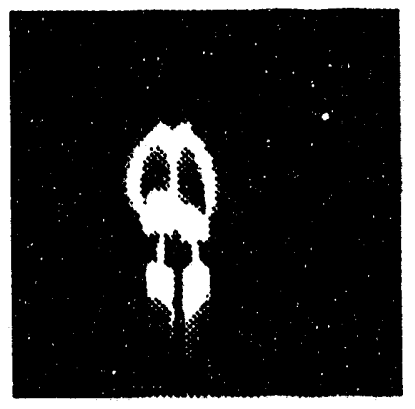

480

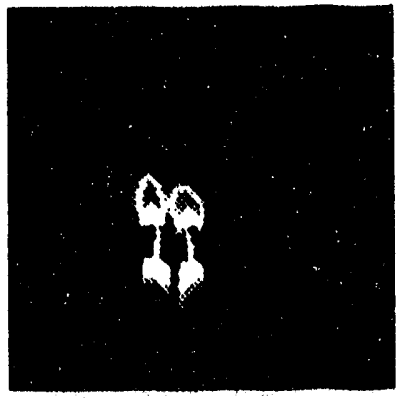

90

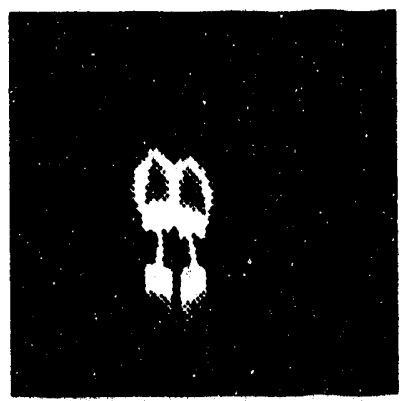

800

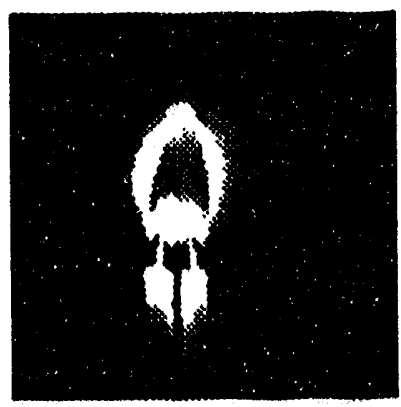

480

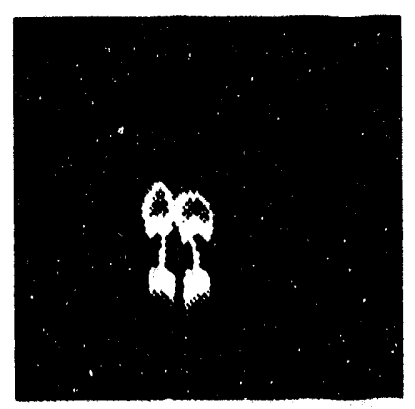

180

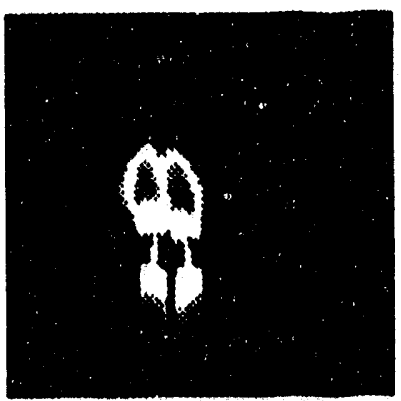

860

Figure 21a. The Burning Sequence for Two-Candle Array at $l / a=6 \mathrm{~mm}$ 


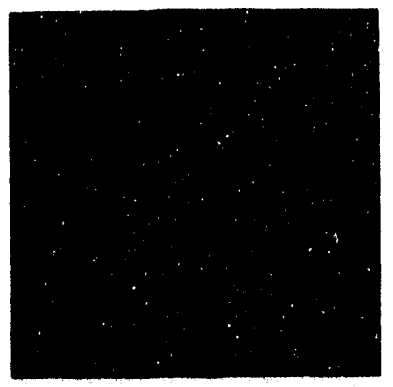

30

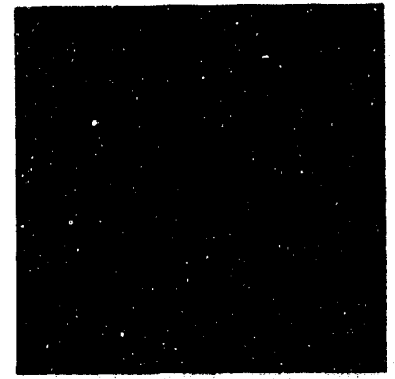

240

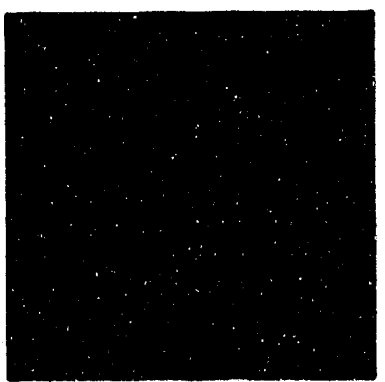

420

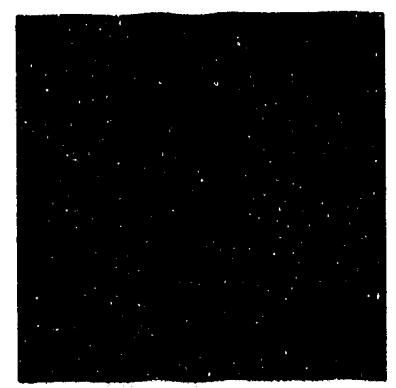

90

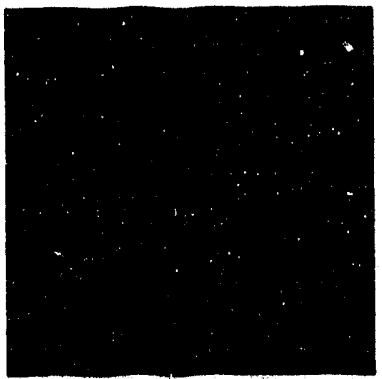

300

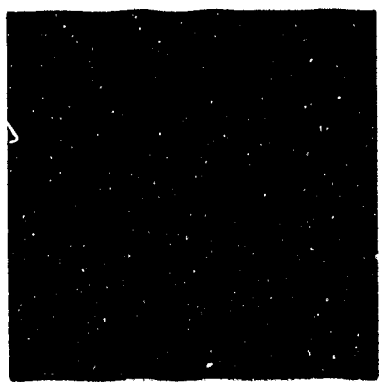

450

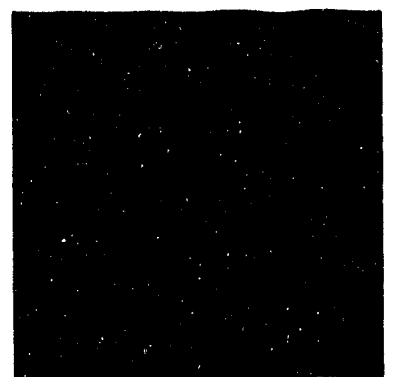

180

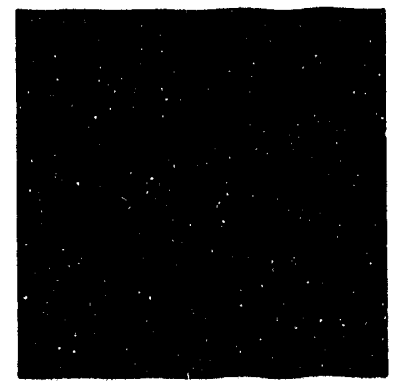

360

Figure 21b. Images of the Burning Sequence for Two-Candle Array with Boundary Detection Function 
msec. It is also interesting to note that the black inner zone discussed in the case of an isolated candle was observed here as well. The inner zone of each candle grew larger as more time elapsed until they merged and reached their largest size as the flames merged and formed a common flame. The inner zone is considerably larger and elongated due to conviction.

\section{VI.3 Isolated Coal Particles}

Experimental studies conducted on char and coal particles using the flat flame burner facility described in chapter V include: high volatile coal (PSOC 1447), medium volatile coal (PSOC 1336), and low volatile coal (PSOC 1516), with special emphasis on the high volatile coal since it is a non-swelling coal. Table 2 summarizes the physical and chemical analysis of coal. The base case and parametric conditions are summarized in Table 3.

\section{VI.3.1 High Volatile Coal (PSOC 1447)}

Once the particle is subjected to the hot gases provided by the flat fl ume burner, the particle heats up rapidly, then ignition is observed to occur heterogeneously at the surface of the particle and more specifically at the particle's leading edge. Figure 22 shows a typical sketch of combustion of coal particle in a vitiated environment. Typically the Reynolds number $(\mathrm{Re})$ is of order 1.5. Images illustrating the sequence of the combustion process are shown in Figure 23. Heterogeneous ignition time was recorded as $360 \mathrm{~ms}$ from the time of exposing the particle to the hot environment. At 
Table. 2 Physical and Chemical Analysis of Coal

\begin{tabular}{|c|c|c|c|}
\hline 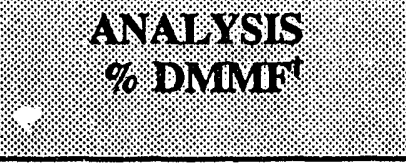 & $\begin{array}{l}\text { lifgh Volatilo } \\
\text { ISOC-144\% }\end{array}$ & 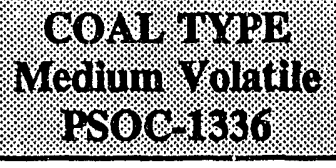 & 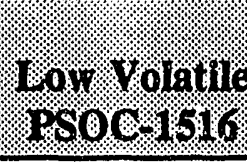 \\
\hline \multicolumn{4}{|l|}{ PROXIMATE } \\
\hline Moisture & - & - & - \\
\hline Ash & - & - & - \\
\hline Volatile & 47.59 & 37.28 & 18.55 \\
\hline Fixed Carbon & 52.41 & 62.72 & 81.45 \\
\hline \multicolumn{4}{|l|}{ ELEMENTAL } \\
\hline Carbon & 77.22 & 87.27 & 90.51 \\
\hline Hydrogen & 5.60 & 5.39 & 4.63 \\
\hline Nitrogen & 1.31 & 1.63 & 1.67 \\
\hline Organic Sulfur & 0.18 & 1.03 & 0.89 \\
\hline Oxygen & 15.69 & 4.67 & 2.30 \\
\hline \multicolumn{4}{|l|}{ ATOMIC RATIOS } \\
\hline $\mathrm{H} / \mathrm{C}$ & 0.8710 & 0.740 & 0.614 \\
\hline $\mathrm{O} / \mathrm{C}$ & 0.1525 & 0.040 & 0.019 \\
\hline $\mathrm{N} / \mathrm{C}$ & 0.0146 & 0.016 & 0.016 \\
\hline S/C & $8.741 \times 10^{-4}$ & $4.43 \times 10^{-3}$ & $3.69 \times 10^{-3}$ \\
\hline STOIC. $\mathrm{O}_{2}\left(\nu_{\mathrm{O}_{2}}\right)$ & 2.35 & 2.72 & 2.77 \\
\hline $\begin{array}{c}\text { FREE SWELLING } \\
\text { INDEX } \\
\end{array}$ & 0.0 & 7.5 & 8.5 \\
\hline $\begin{array}{c}\text { HEATING VALUE } \\
(\mathrm{KJ} / \mathrm{kg})\end{array}$ & $32,018.34$ & $36,630.06$ & $37,138.11$ \\
\hline
\end{tabular}

${ }^{\dagger}$ Dry mineral matter free

EMPIRICAL FORMULA: $\mathrm{CH}_{.87} \mathrm{O}_{.15} \mathrm{~N}_{.014} \mathrm{~S}_{.0008} \mathrm{CH}_{.74} \mathrm{O}_{.04} \mathrm{~N}_{.016} \mathrm{~S}_{.0044} \mathrm{CH}_{.61} \mathrm{O}_{.019} \mathrm{~N}_{.016} \mathrm{~S}_{.00369}$ 
Table 3. Base Case and Parametric Conditions for Isolated Char/Coal Particles

\section{BASE CASE}

High Volatile Coal Particles (PSOC 1447)

Temperature $T_{\infty}=1027 \mathrm{~K}$

Oxygen Concentration $Y_{o 2}=12.4 \%$

Diameter $d_{p}=0.80 \mathrm{~mm}$

PARAMETRTC CONBMIONS

Medium Volatile Coal Particles (PSOC 1336)

Diameter $d_{p}=0.84 \mathrm{~mm}$

Low Volatile Coal Particles (PSOC 1516)

Diameter $d_{p}=0.81 \mathrm{~mm}$

Char Particles

Diameter $d_{p}=1.0 \mathrm{~mm}$ 


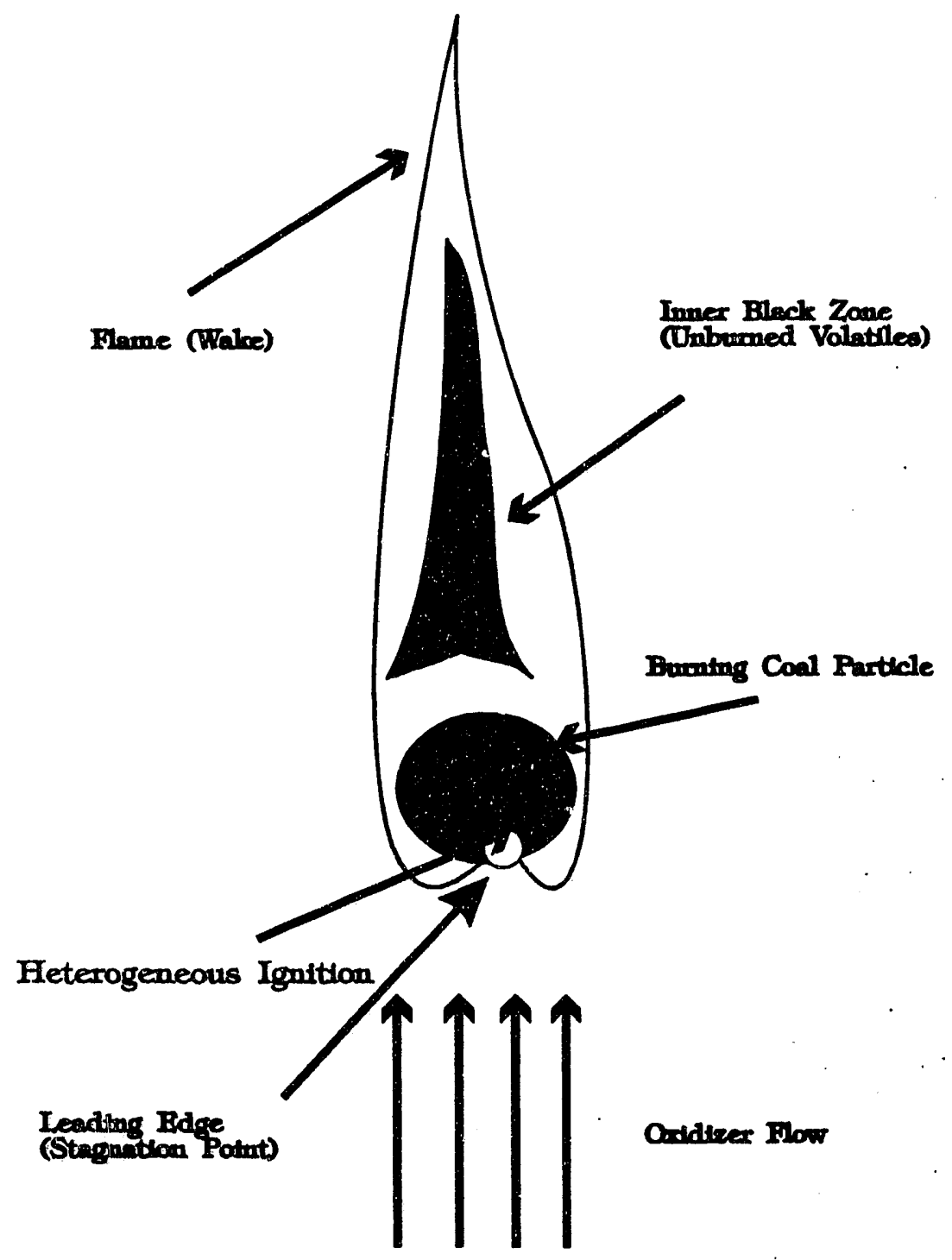

Figure 22. A Typical Sketch of Combustion of Isolated Coal Particle in Vitiated Environment 

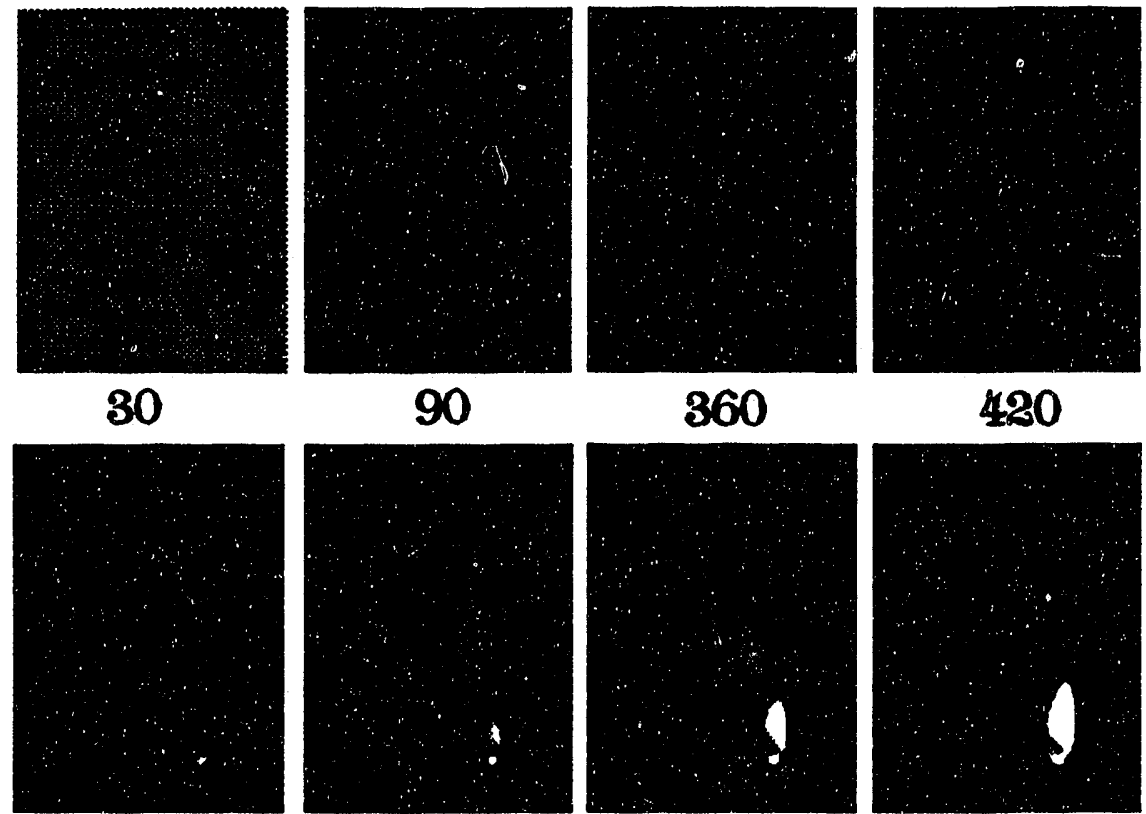

450

480

510

540
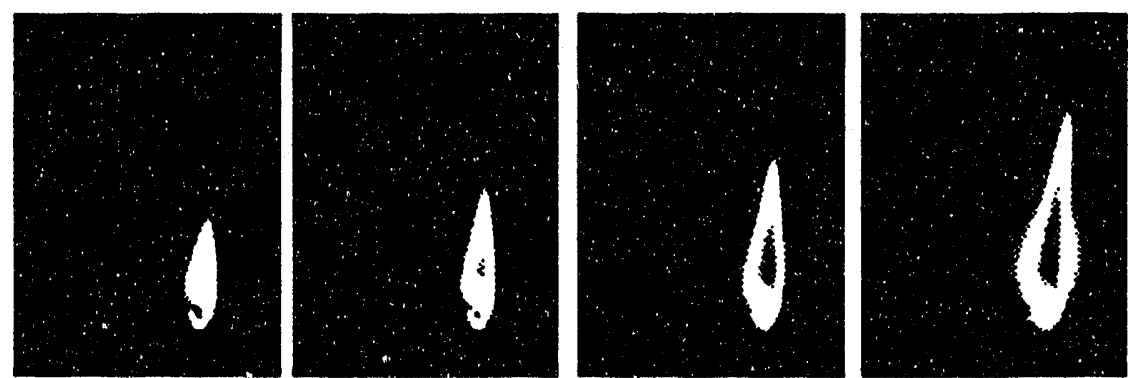

570

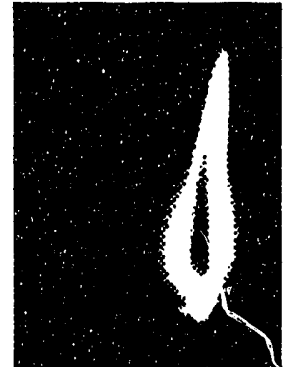

600

630

660

690

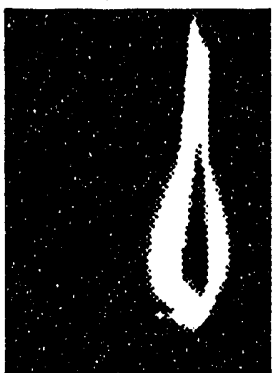

720

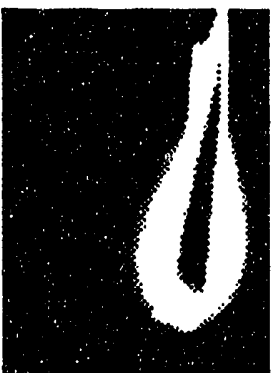

750

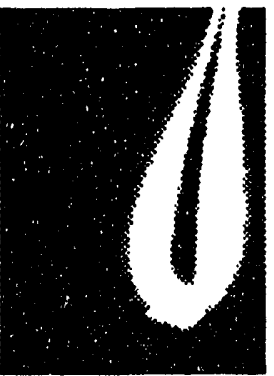

780

Figure 23a. Ignition and Combustion Sequence for High Volatile Isolated Coal Particle (Time in msec) 

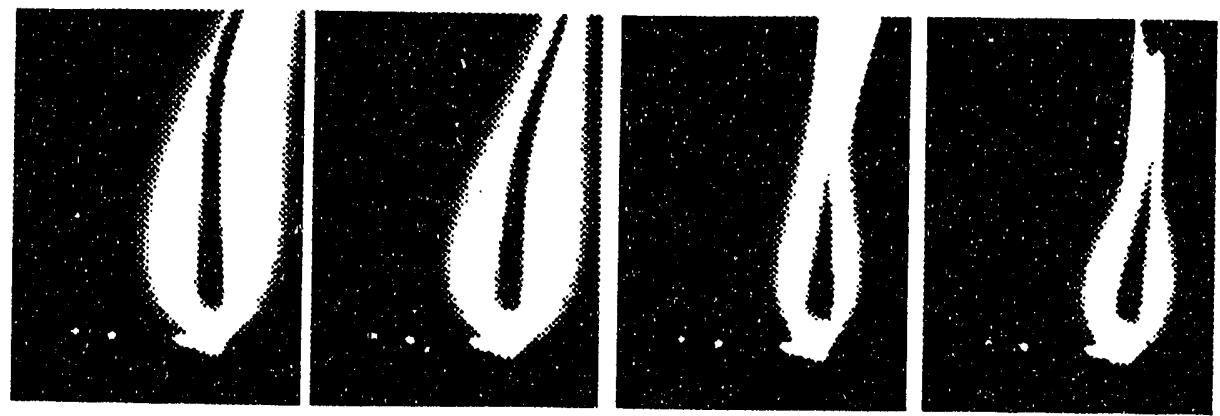

870

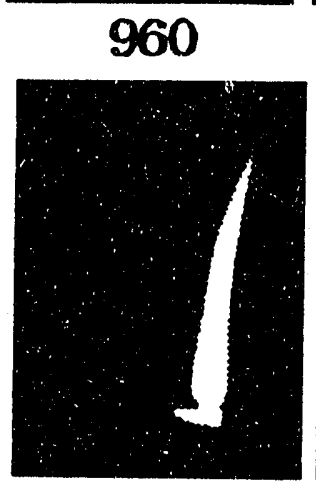

990

1020

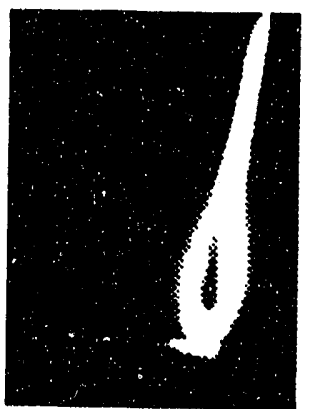

1050

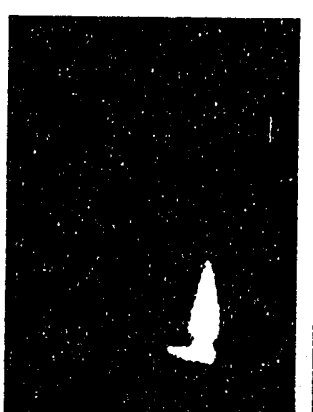

1110

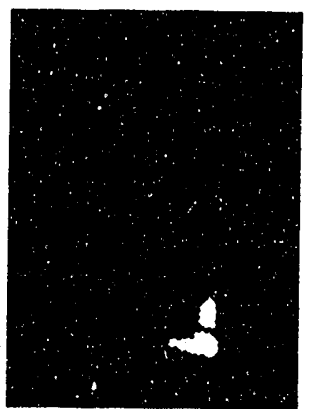

1140

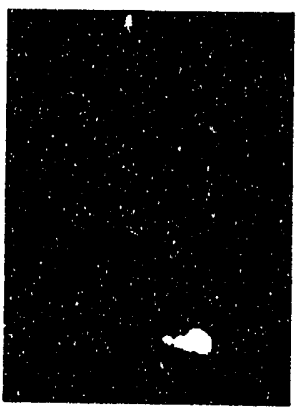

1170

Figure 23a. (Continued) 

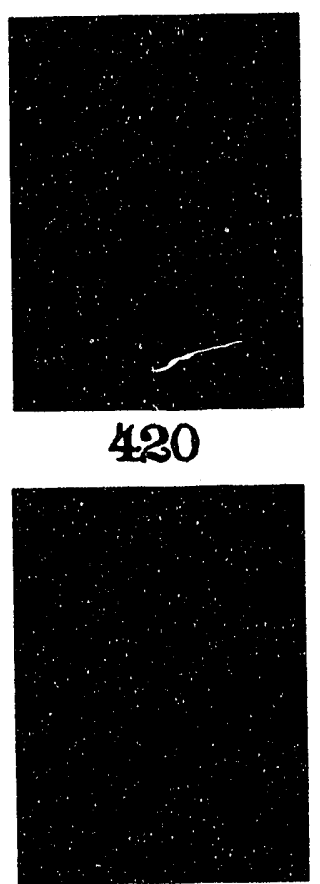

540

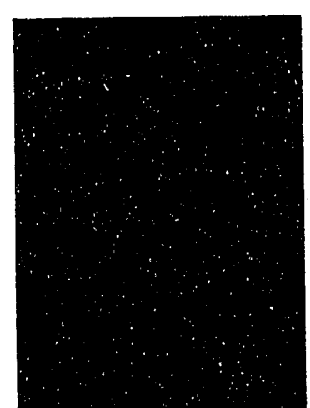

660

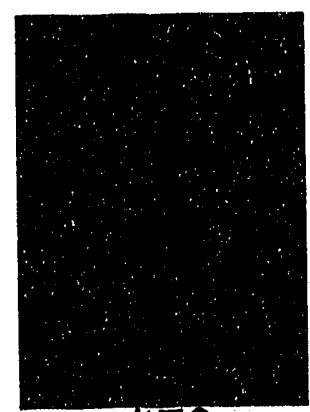

450

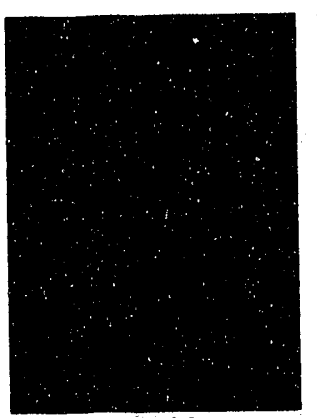

570

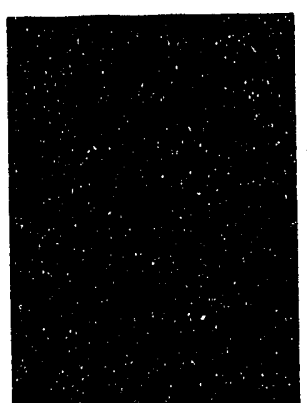

660

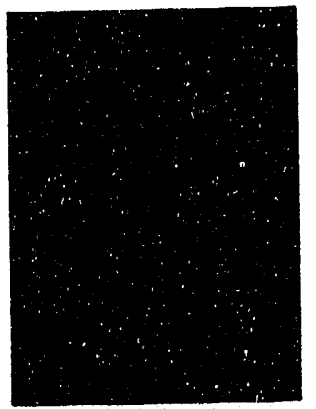

480

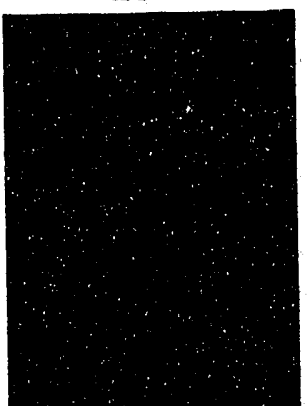

600

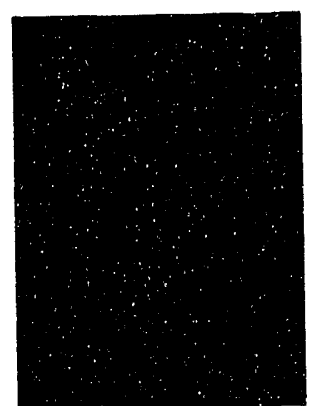

690

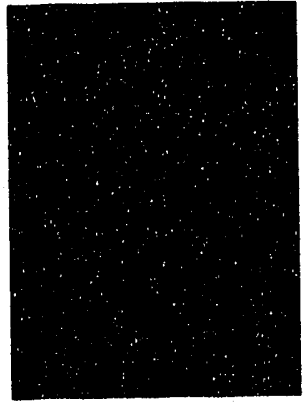

510

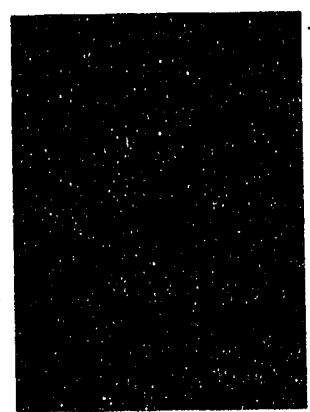

630

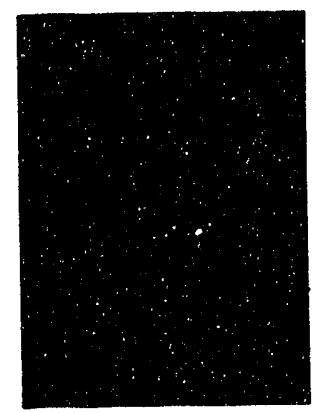

750

Figure 23b. Images of Ignition and Combustion Sequence with Boundary Detection Function for High Volatile Isolated Coal Particle 

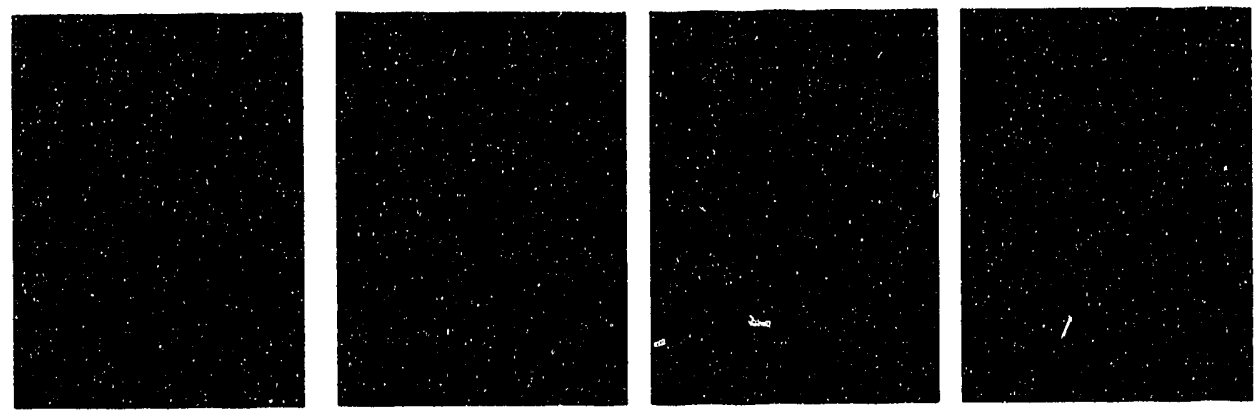

870
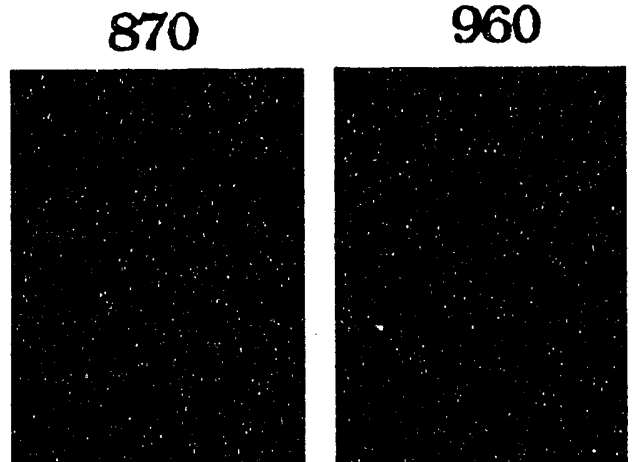

990

1080

1050
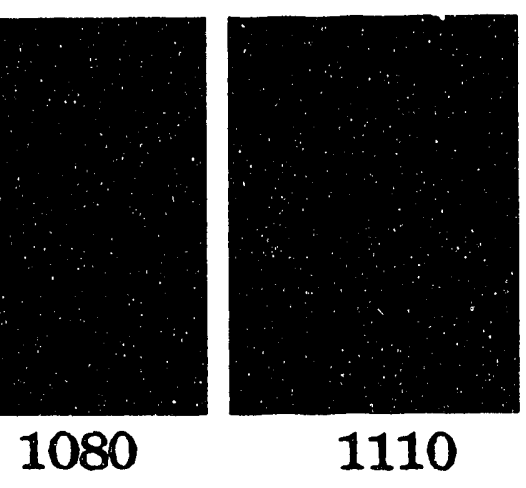

1110

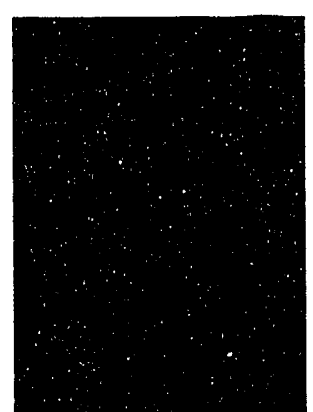

1140

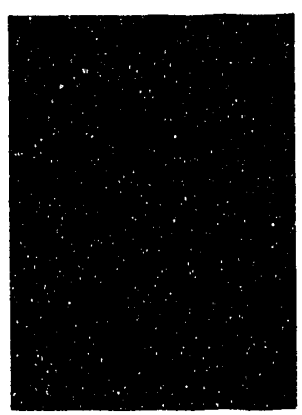

1170

Figure 23b. (Continued) 
$450 \mathrm{~ms}$, volatile seem to be ejected upwards as jets from the particle in the direction of the convective flow (Reynold number, $\mathrm{Re}=1.57$ and Nusselt number, $\mathrm{Nu}=2.60$ ), and burned in the gas phase (homogeneous ignition). Since the particle is not exactly spherical, the convective flow is not symmetric around the particle. It is interesting to note that as the coal burned, volatiles continued to burn homogeneously in the gas phase, but only in the wake of the particle; since oxygen is available at the leading edge heterogeneous combustion also occurs. As more time elapsed, both ignition processes proceeded in parallel. Eventually, all volatile were exhausted and the combustion process was completed at $1170 \mathrm{~ms}$, leaving a particle consisted mainly of ash.

It is worth mentioning that grey streaks were observed in the images. This can be possibly attributed to the ejection of volatile; no gray streaks were observed prior to ignition. If the volatile evolve first, they may form a detached flame; further, they may blow the oxygen from the surface preventing heterogeneous ignition at the leading edge. However, these phenomena was not observed. This suggests that heterogeneous ignition occurred first.

As more time elapses and more heat is supplied from the vitiated gases to the particle, the particle partially pyrolyzes releasing volatile. The volatile diffuse into the surrounding atmosphere, and then convected to the wake of the particle. Since the combustion was not intense yet at $450 \mathrm{~ms}$ and the volatile liberation occurred as jets from the pores, oxygen could still reach the particle's surface and heterogeneous combustion of fixed carbon and in situ volatile matter could proceed in parallel with gas 
phase combustion, particularly at the leading edge. Occurrence of the heterogeneous ignition was a striking finding.

Typically, homogeneous ignition is favored for high volatile coal in a quiescent atmosphere and for large particles $\left(d_{p}>1.0 \mathrm{~mm}\right)$, since a flammable mixture can be readily formed in the gas phase surrounding the particle. However, the behavior of this particle, although it falls in the large particles range, was the opposite since the volatile were not found to be evolved prior to ignition, further, oxygen could be rapidly transported to the stagnation point, causing heterogeneous ignition.

It is believed that the amount of volatile matter, flammability of the volatile and transport rate from the gas to the particle and vice versa determine whether ignition of an isolated coal particle occurs heterogeneously or homogeneously.

It is interesting to note that once the particle was ignited, volatile did not start to eject until after about $120 \mathrm{~ms}$ from ignition time. Volatile ejected upwards and started to burn in the wake. The flame formed mainly by volatile matter, started small and grew to larger size as the time increased. Figure 24 shows the typical flame geometry. Measurements were taken for flame height, width, inner zone height and width (Table 4). Tip velocity was also estimated by measuring $H_{p}$ versus time. The flame detached from the particle in the early stages of the combustion process and reattached later as more volatile are released and as the combustion is intensified.

Examining the flame formation and its characteristics, many observations were noted (Figure 25). It is noted that $\mathrm{H}_{\mathrm{p}}, \mathrm{H}_{\mathrm{l}}, \mathrm{W}_{\mathrm{F}}$, and $\mathrm{W}_{\mathrm{l}}$, increase with time. After about 


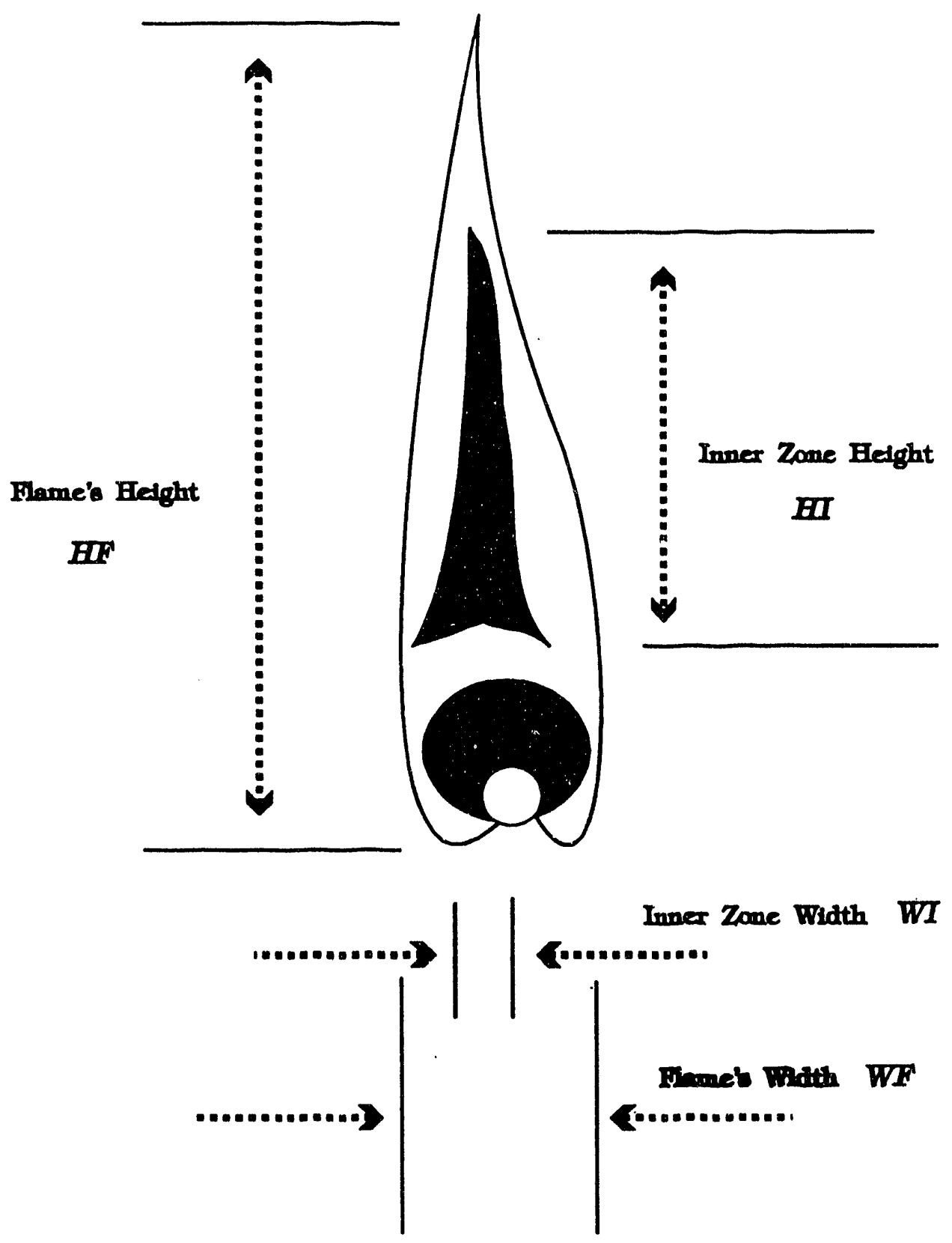

Figure 24. A Typical Flame Geometry of Isolated High Volatile Coal Particle 
Table 4. Flame Characteristics of High Volatile Isolated Coal Particle

Particle Diameter $=0.80 \mathrm{~mm}$

Ignition Time $=360 \mathrm{~ms}$

Combustion Time $=1170 \mathrm{~ms}$

\begin{tabular}{|c|c|c|c|c|c|c|}
\hline Trame & $\begin{array}{l}\text { Glapsed: } \\
\text { I Iime : } \\
\text { (ms): }\end{array}$ & $\begin{array}{l}\text { Tlame } \\
\text { Height } \\
\text { (minm) }\end{array}$ & $\begin{array}{l}\text { Flarne } \\
\text { Widith } \\
\text { (mimin) }\end{array}$ & Timer Zone & 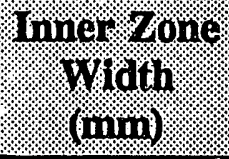 & 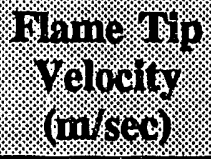 \\
\hline 1 & 30 & $-\neq$ & - & - & - & - \\
\hline 2 & 60 & - & - & - & - & - \\
\hline 3 & 90 & - & - & - & - & - \\
\hline 4 & 120 & - & - & - & - & - \\
\hline 5 & 150 & - & - & - & - & - \\
\hline 6 & 180 & - & - & - & - & - \\
\hline 7 & 210 & - & - & - & - & - \\
\hline 8 & 240 & - & - & - & - & - \\
\hline 9 & 270 & - & - & - & - & $=$ \\
\hline 10 & 300 & - & - & - & - & - \\
\hline 11 & 330 & - & - & - & - & - \\
\hline 12 & 360 & - & - & - & - & - \\
\hline 3 & 390 & - & - & - & - & - \\
\hline 14 & 420 & - & - & - & - & - \\
\hline 15 & 450 & 2.09 & 0.44 & - & - & 0.70 \\
\hline 16 & 480 & 3.17 & 1.33 & - & - & 0.036 \\
\hline 17 & 510 & 4.05 & 1.77 & - & - & 0.029 \\
\hline 18 & 540 & 5.76 & 1.96 & - & - & 0.057 \\
\hline 19 & 570 & 7.66 & 2.15 & 1.14 & 0.44 & 0.063 \\
\hline 20 & 600 & 9.69 & 2.6 & 3.48 & 0.89 & 0.068 \\
\hline
\end{tabular}


Table 4. (Continued)

\begin{tabular}{|c|c|c|c|c|c|c|}
\hline Irame & $\begin{array}{l}\text { Filapsed } \\
\text { Tume: } \\
\text { (ms) }\end{array}$ & 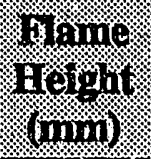 & 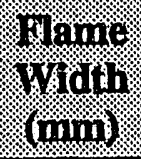 & 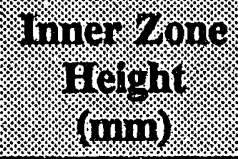 & Imerigone & 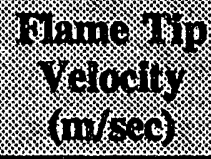 \\
\hline 21 & 630 & 12.59 & 3.55 & 5.51 & 1.14 & 0.097 \\
\hline 22 & 660 & 15.57 & 4.31 & 6.83 & 1.08 & 0.099 \\
\hline 23 & 690 & 17.92 & 4.94 & 8.35 & 1.58 & 0.078 \\
\hline 24 & 720 & 18.1 & 6.52 & 13.04 & 1.71 & 0.006 \\
\hline 25 & 750 & $\mathrm{NA}^{+}$ & 6.96 & 16.20 & 1.52 & NA \\
\hline 26 & 780 & NA & 7 & NA & 1.5 & NA \\
\hline 27 & 810 & NA & 7.07 & NA & 1.49 & NA \\
\hline 28 & 840 & NA & 7.11 & NA & 1.46 & NA \\
\hline 29 & 870 & NA & 7.15 & NA & 1.45 & NA \\
\hline 30 & 900 & NA & 7.09 & NA & 1.4 & NA \\
\hline 31 & 930 & NA & 7.05 & NA & 1.38 & NA \\
\hline 32 & 960 & NA & 7.03 & NA & 1.33 & $\mathbf{N A}$ \\
\hline 33 & 990 & NA & 4.55 & 7.6 & 1.37 & NA \\
\hline 34 & 1020 & NA & 4.94 & 8.35 & 1.33 & NA \\
\hline 35 & 1050 & NA & 3.86 & 4.18 & 0.76 & NA \\
\hline 36 & 1080 & 14.12 & 2.09 & 0 & 0 & NA \\
\hline 37 & 1110 & 5.26 & 1.84 & 0 & 0 & -0.295 \\
\hline 38 & 1140 & 2.78 & 1.2 & 0 & 0 & -0.083 \\
\hline 39 & 1170 & 0 & 0 & 0 & 0 & -0.093 \\
\hline
\end{tabular}

* Flame did not form yet

+ Flame exceeded monitor's screen view 


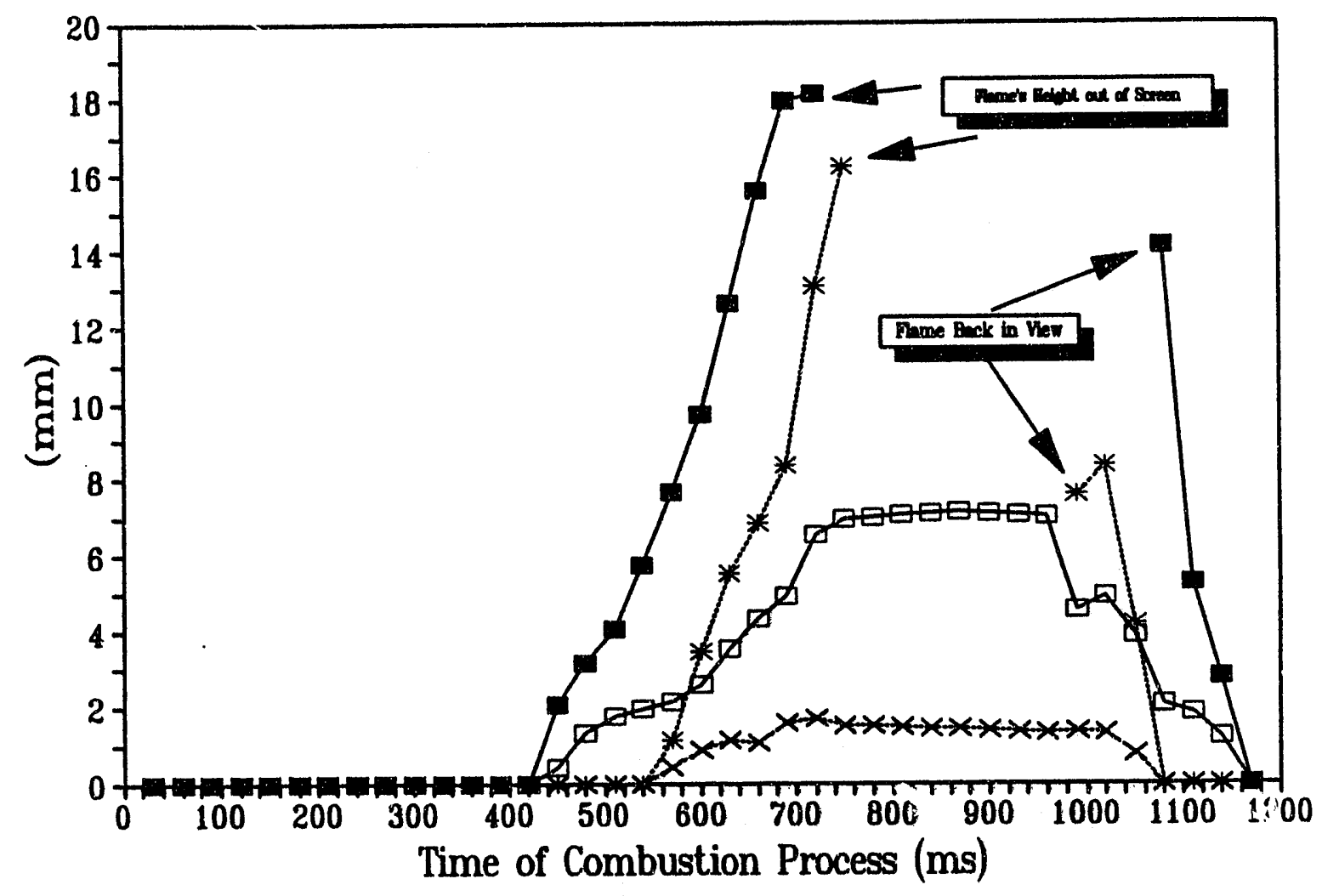

- Plame-Height $\quad$ - Flame-Width $\quad *-$ Inner Zone-Height $\leftrightarrow$ Inner-Zone Width

Figure 25. Flame Characteristics of Isolated High Volatile Coal Particle 
$800 \mathrm{msec}$, they level off, and as the volatile are depleted, they decrease again. As the flame grew, a black zone inside the flame was formed. The formation of the inner zone occurred at $570 \mathrm{~ms}$ and grew larger as the flame became larger and larger. Figure 26 shows the flame tip velocity as a function of time. The black inner zone can be attributed to the volatile rich core while the outer bright flame corresponds to thick diffusion flame. In addition, as more time elapsed, the flame's height exceeded the monitor's screen view. Since the inner zone was restricted by the flame's behavior, it also grew and exceeded the monitor's view. Towards the end of the combustion process, at $1080 \mathrm{~ms}$, the inner zone disappeared since ejected volatile decreased drastically, leaving the flame to burn for $90 \mathrm{~ms}$ more before the completion of the combustion process.

With six similar experiments conducted to study the behavior of an isolated particle under combustion, two particles showed an interesting behavior of intermittent ignition. Intermittent ignition is simply a cyclic formation and vanishing of the flame through out the combustion process. Figure 27 shows this behavior. The flame was formed at $540 \mathrm{~ms}$, vanished at $600 \mathrm{~ms}$, formed again at $630 \mathrm{~ms}$, vanished at $720 \mathrm{~ms}$, and eventually formed at $750 \mathrm{~ms}$. The flame formation continued until the end of the combustion process. A possible explanation to this behavior is as follows: as the particle is heated, a thermal wave propagates to the interior of the particle. The volatile liberation rate is controlled by the rate of propagation and rate of heat input for pyrolysis. But, the volatile liberated from the particle are swept a way in the wake 


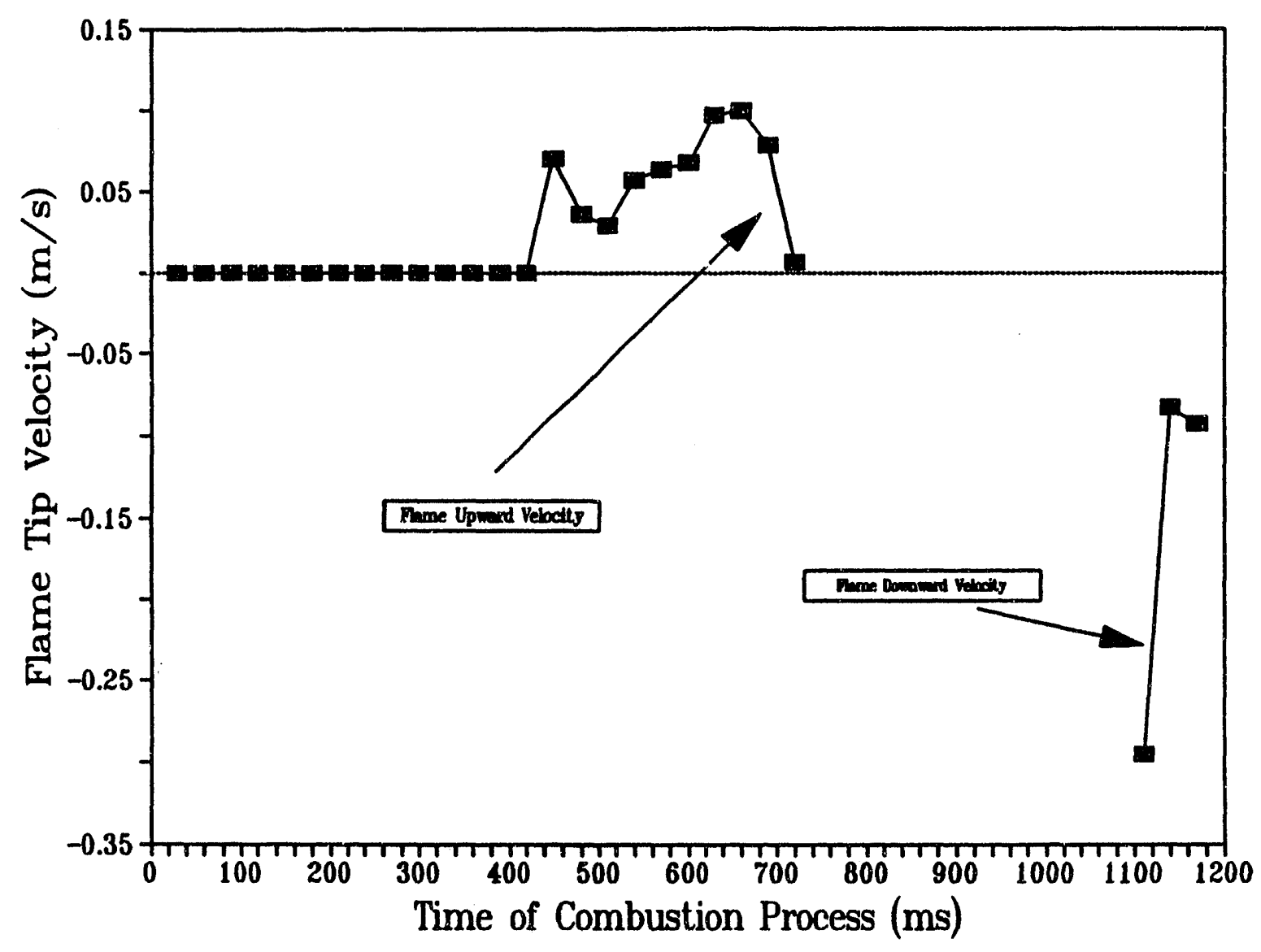

Figure 26. Variation of Flame Tip Velocity with Time for Isolated High Volatile Coal Particle 

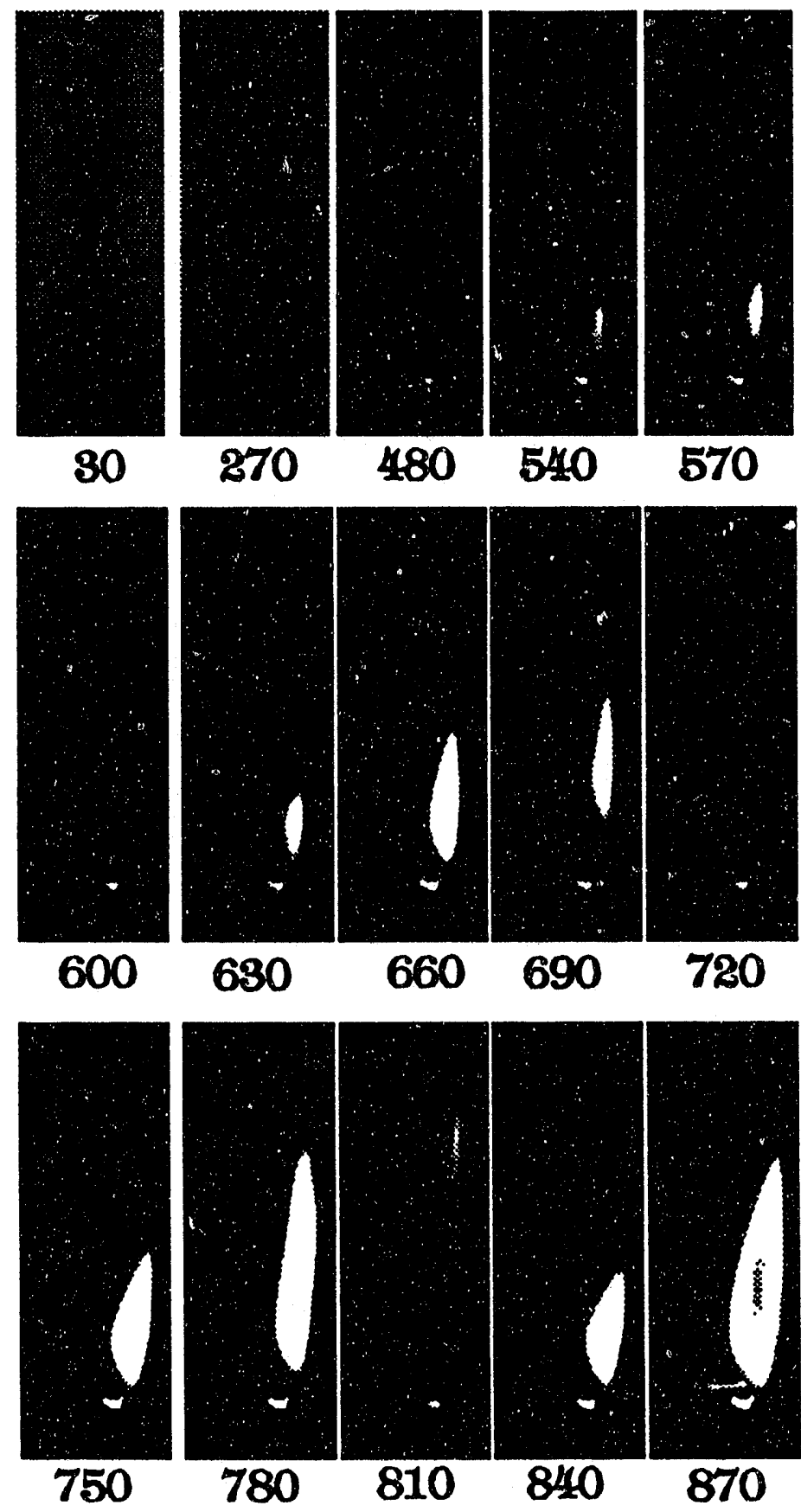

Figure 27a. Intermittent Ignition and Combustion Sequence for High Volatile Coal Particle 


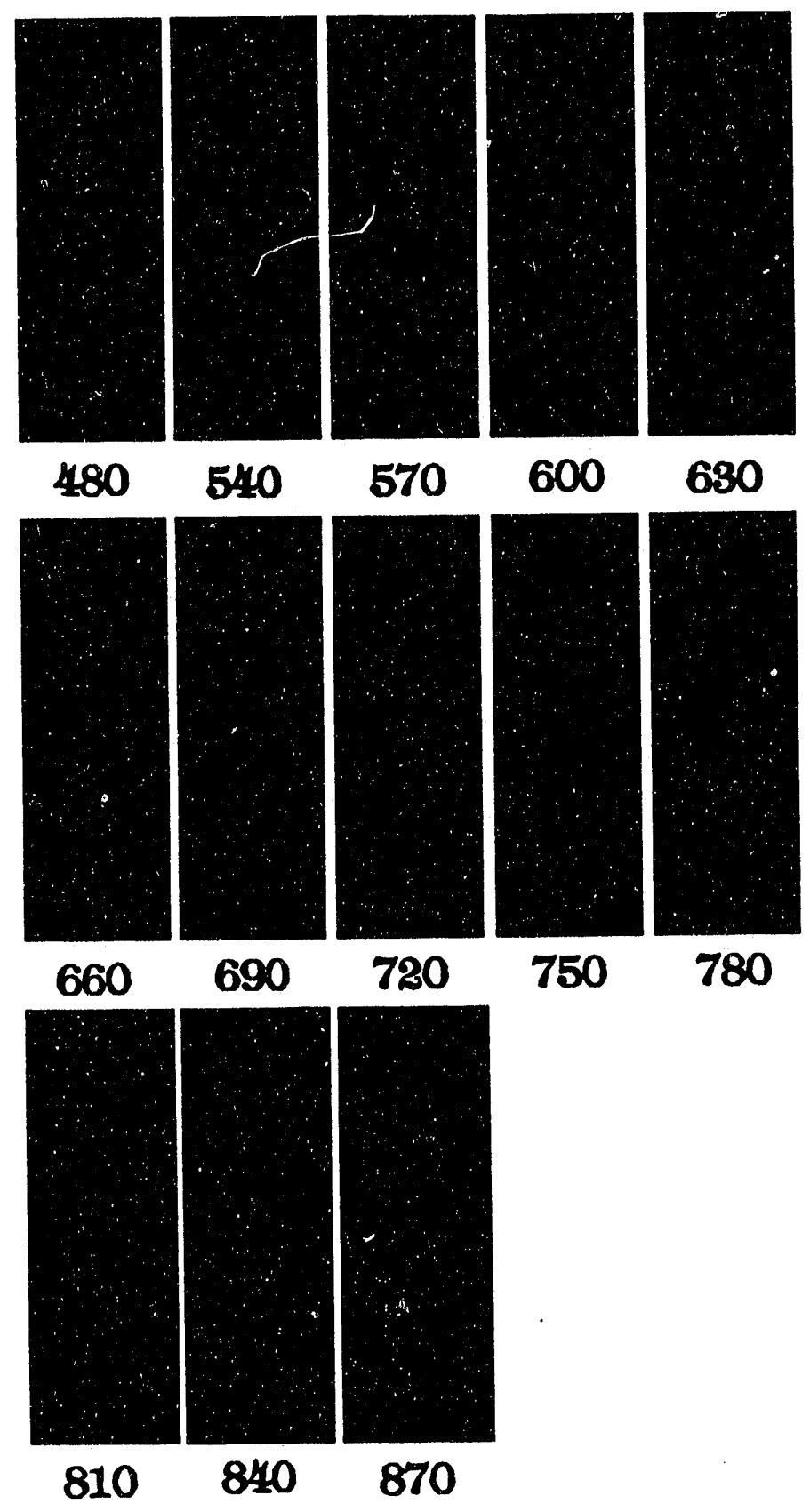

Figure 27b. Images of Intermittent Ignition and Combustion Sequence with Boundary Detection Function 
where they burn rapidly and there is no heat feed back from the volatile flame to the particle. Since volatile combustion rate exceeds the volatile liberation rate, the flame is quenched until the heat input to the particle is sufficient to generate the next batch of volatile. Thus, if the volatile burning rate is more rapid than the liberation rate, then an intermittent volatile ignition and combustion are expected. It should be noted that as the volatile proceed to burn in the gas phase, the leading edge continues to burn heterogeneously.

\section{VI.3.2 Medium Volatile Coal (PSOC 1336)}

Table 2 shows the physical and chemical analysis of coal. Unlike the high volatile particle, this particle ignited just after $30 \mathrm{~ms}$ from introducing it to the hot environment provided by the burner. But, the ignition sequence here resembles that of the high volatile (Figure 28). Ignition started heterogeneously and simultaneously at three different sites on the particle's surface. Volatile are then ejected upwards in the direction of the convective flow ( $\operatorname{Re}=1.65$ and $\mathrm{Nu}=2.65$ ), and started burning in the gas phase (homogeneous ignition). Afterwards, both processes, homogeneous and heterogeneous, proceeded in parallel until the completion of the combustion process. It is worth mentioning that the particle, swelled after combustion and it's size was increased by 2.7 times the original size, and consisted mainly from ash.

In addition, flame characteristics were observed to be similar to the high volatile ones (Figure 29). Flame initiated at $150 \mathrm{~ms}$, and grew larger and larger as time increased. Figure 30 shows the tip velocity as a function of time. Again, the flame 

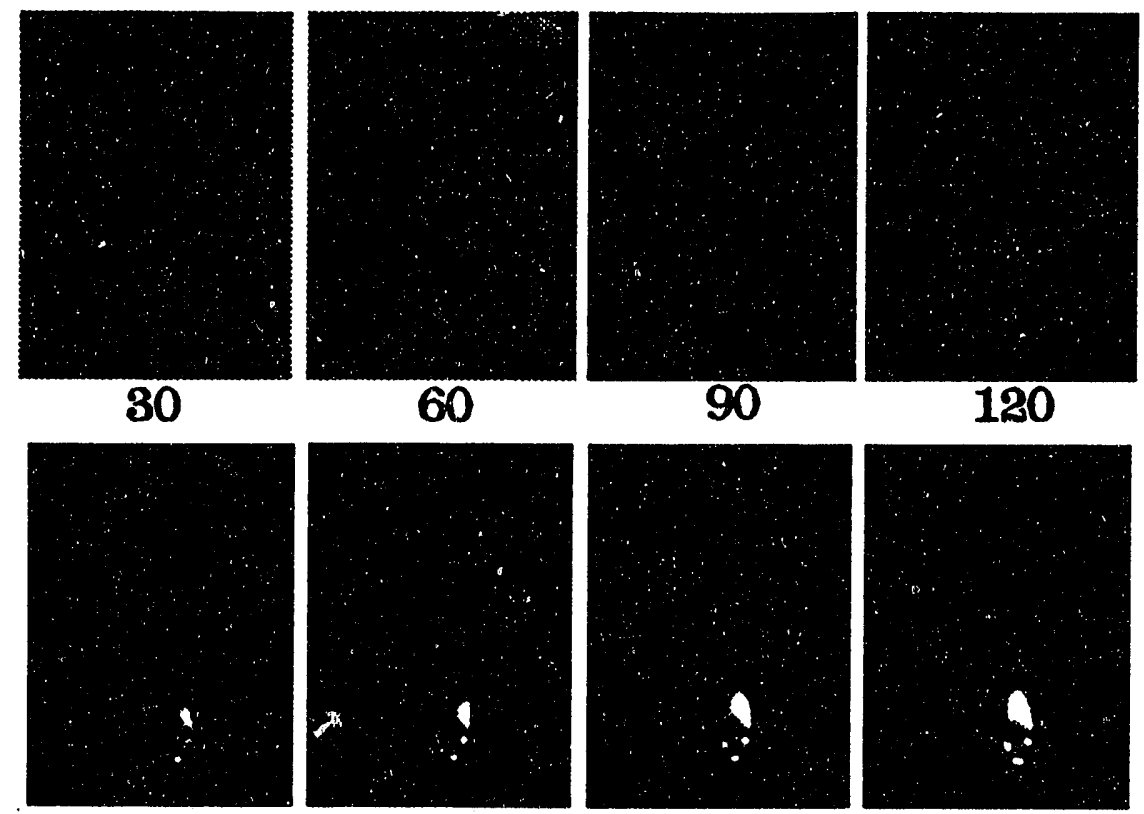

120
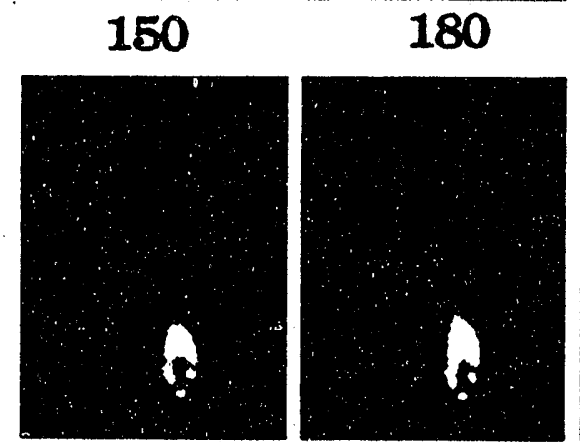

210
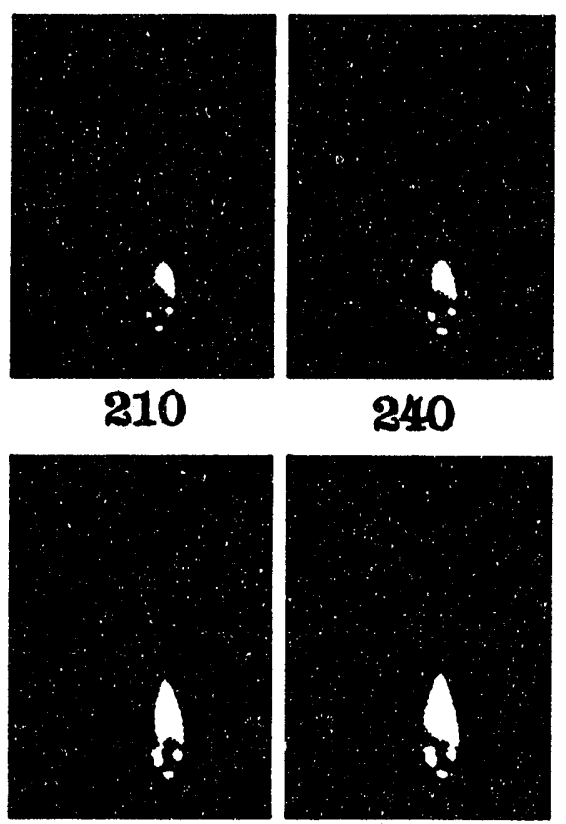

240

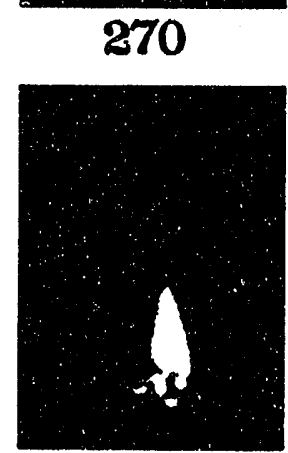

300

330

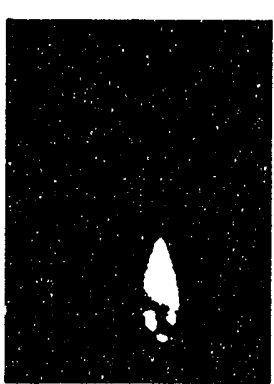

360
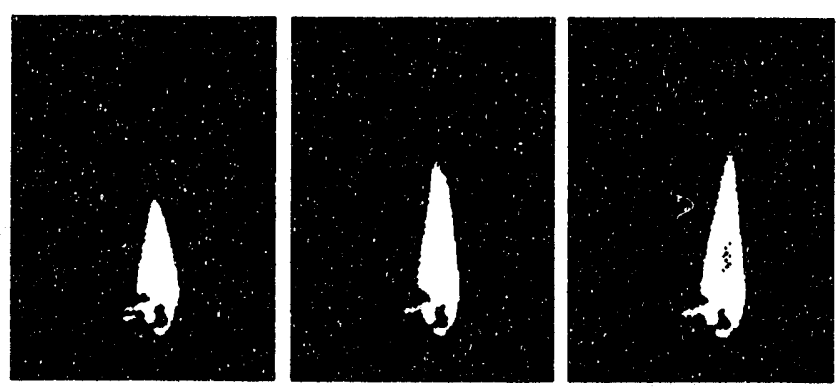

390

420

450

480

Figure 28a. Ignition and Combustion Sequence for Isolated Medium

Volatile Coal Particle 


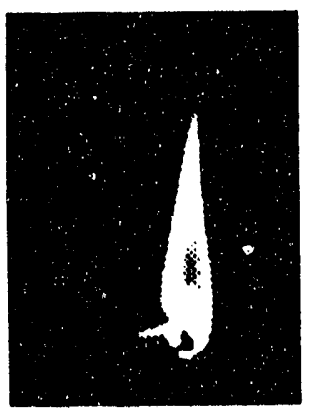

510

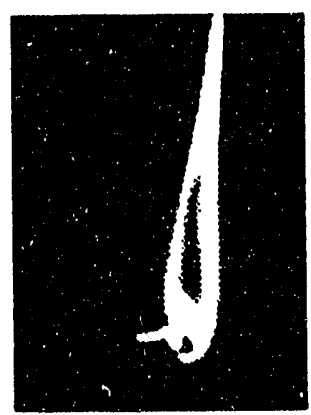

630

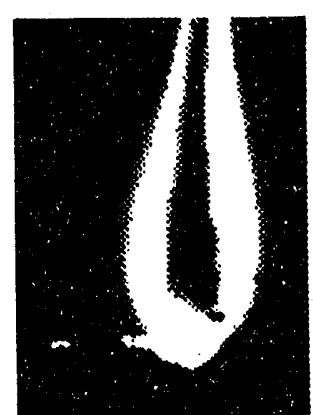

1200

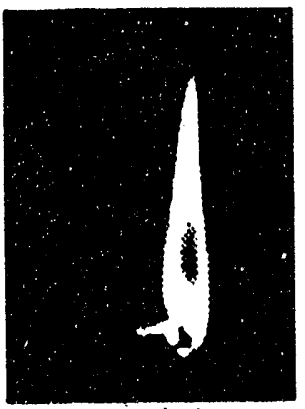

540

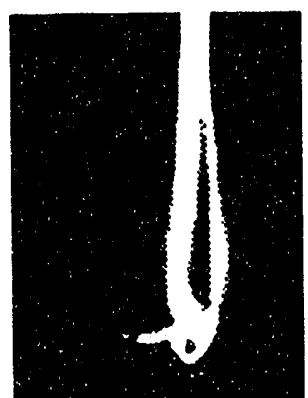

780

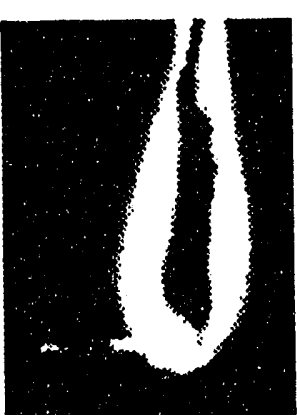

1260

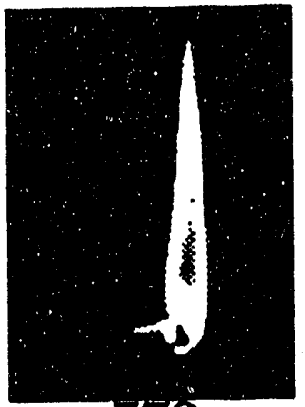

570

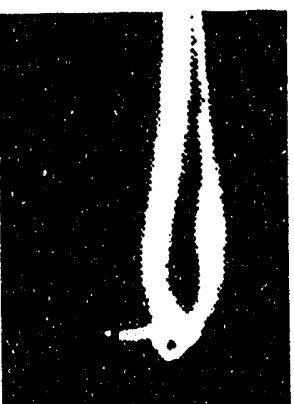

900

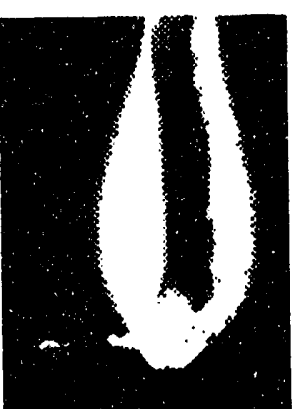

1320

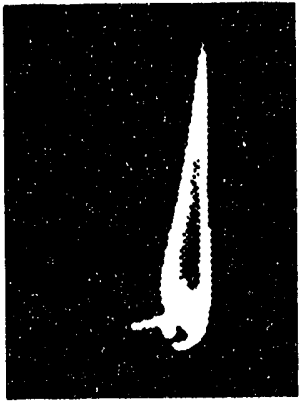

600

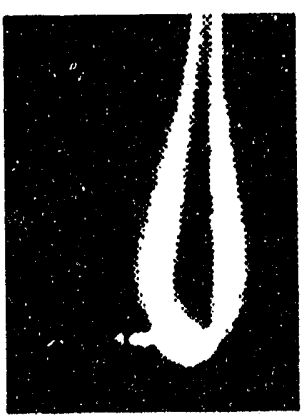

1050

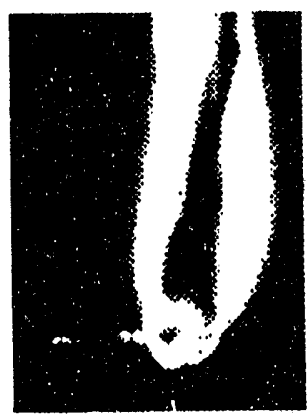

1350

Figure 28a. (Continued) 

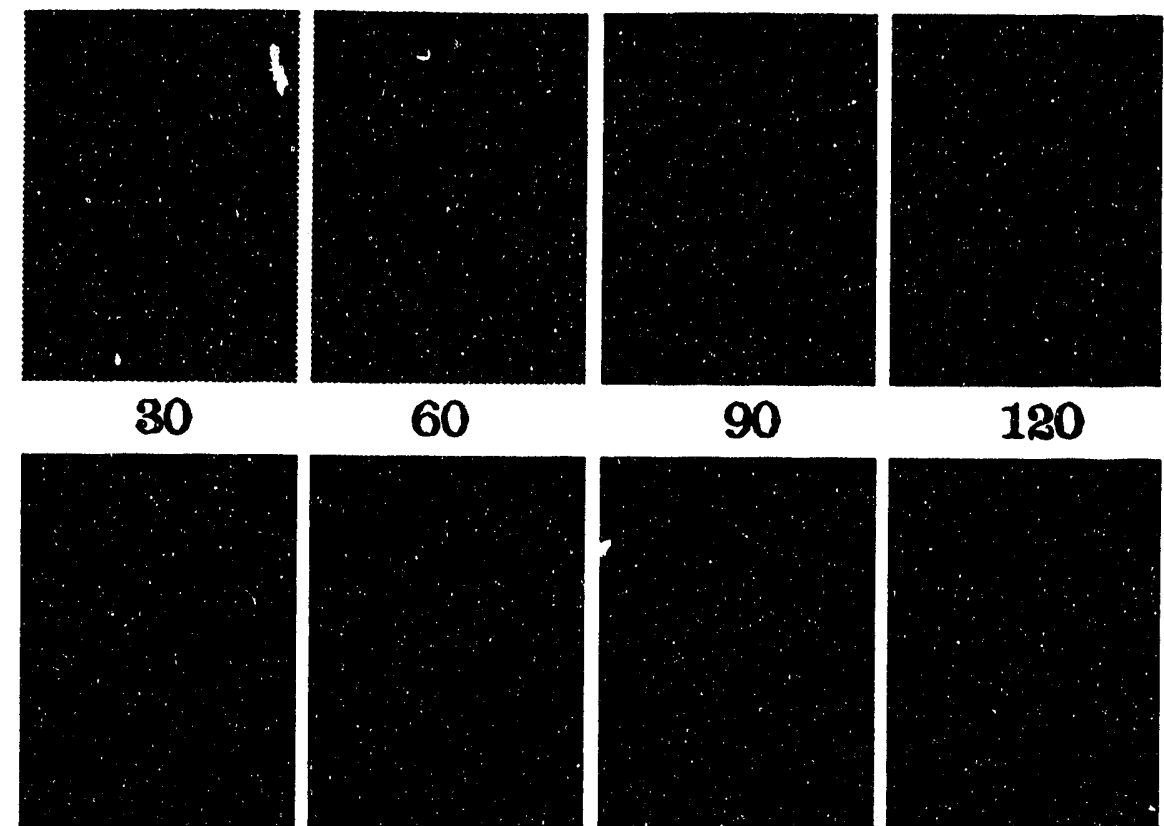

60

90

180
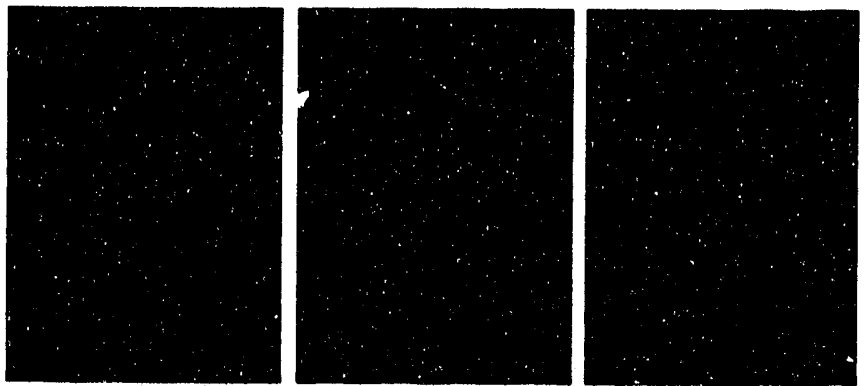

150

180

210

240
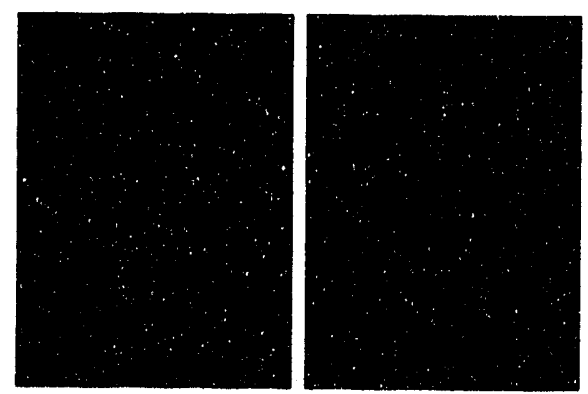

300

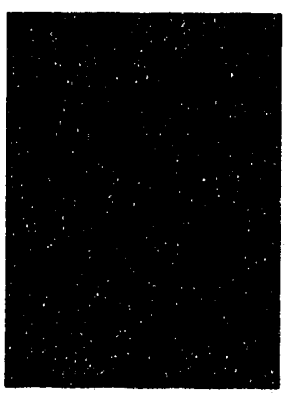

330

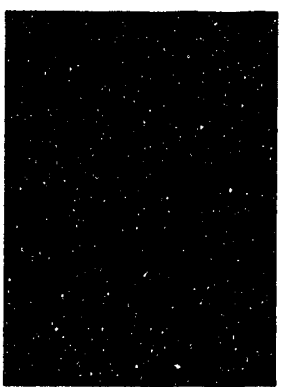

$\mathbf{2 7 0}$
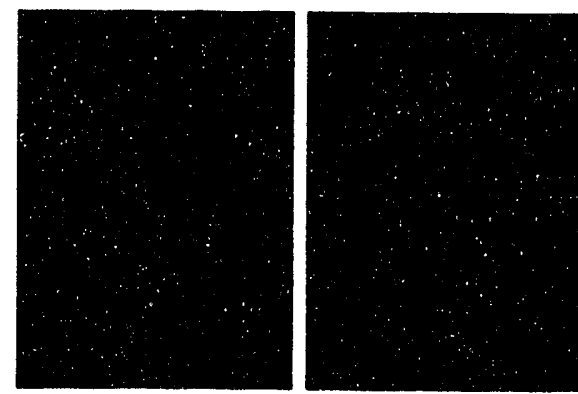

390

420

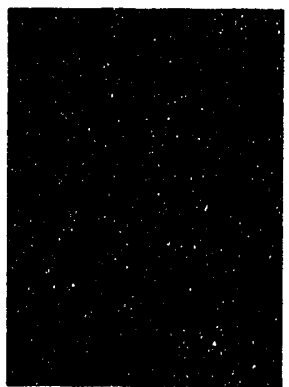

860

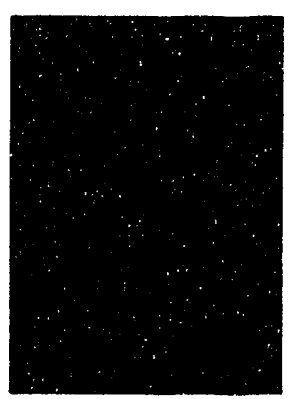

480

480

Figure 28b. Images of Ignition and Combustion Sequence with Boundary Detection Function for Isolated Medium Volatile Coal Particle 

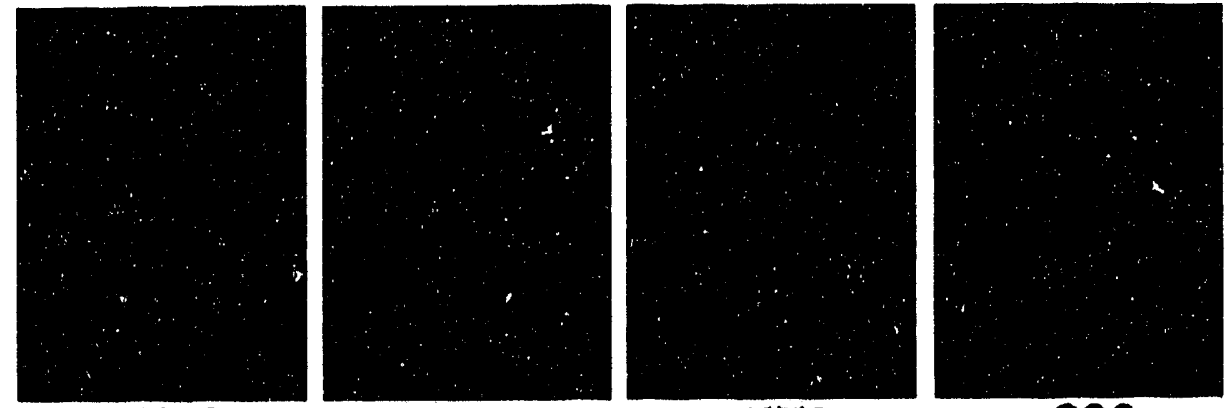

510

540
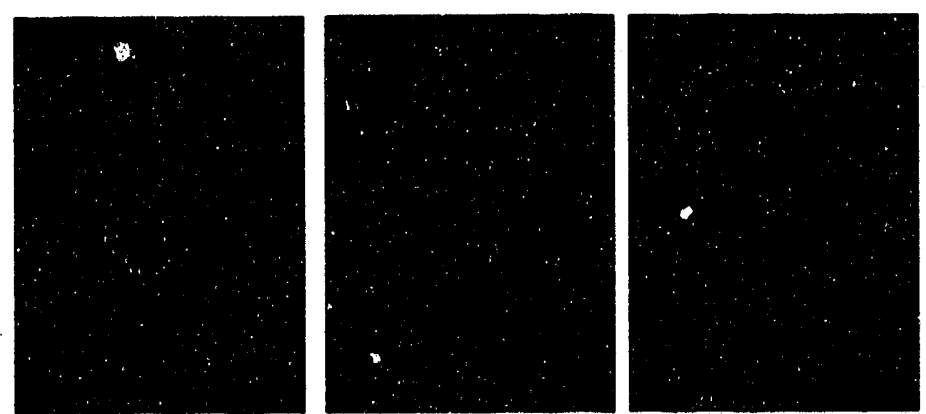

600

630

780

900
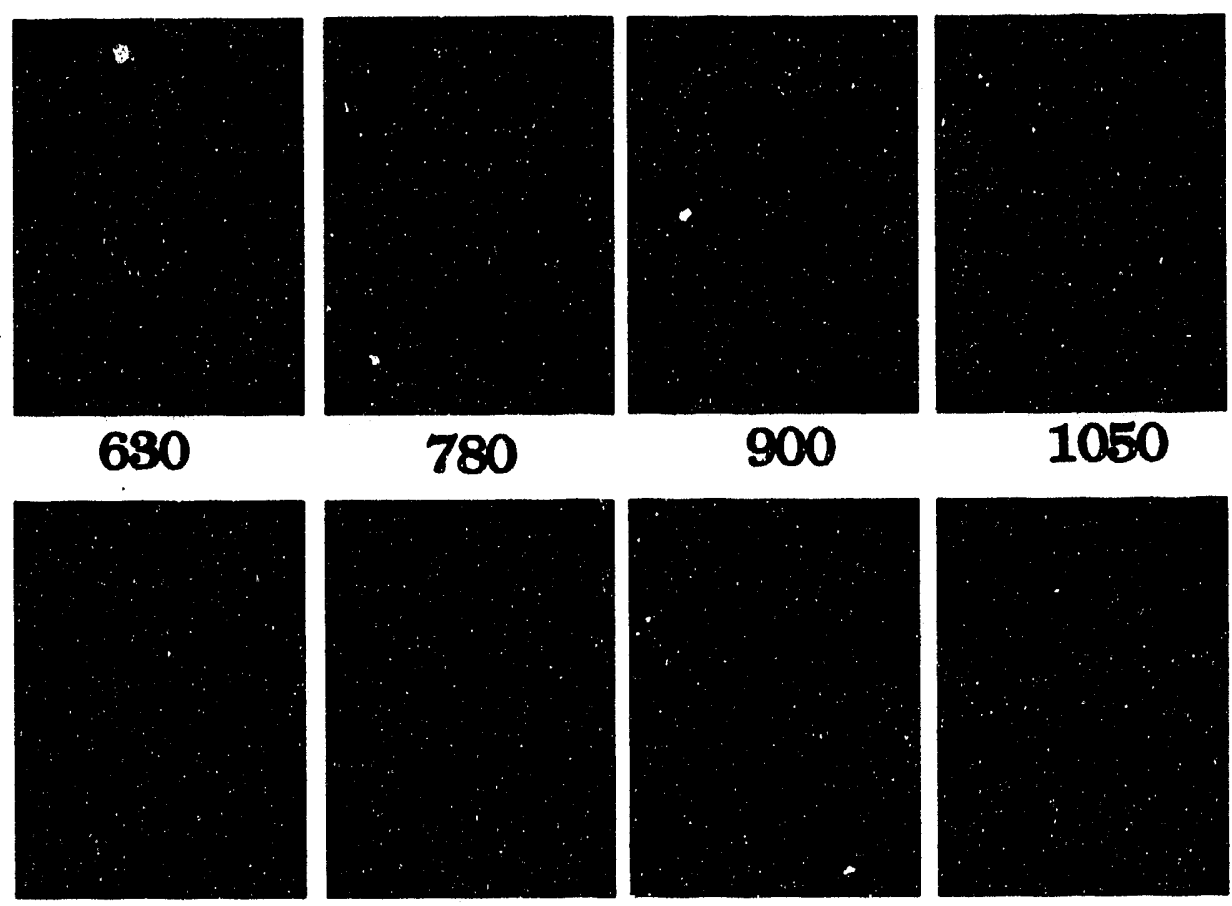

1050

1200

1260

1320

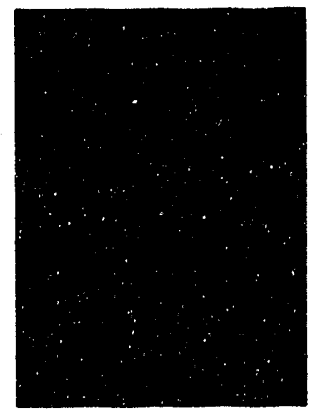

1850

Figure 28b. (Continued) 


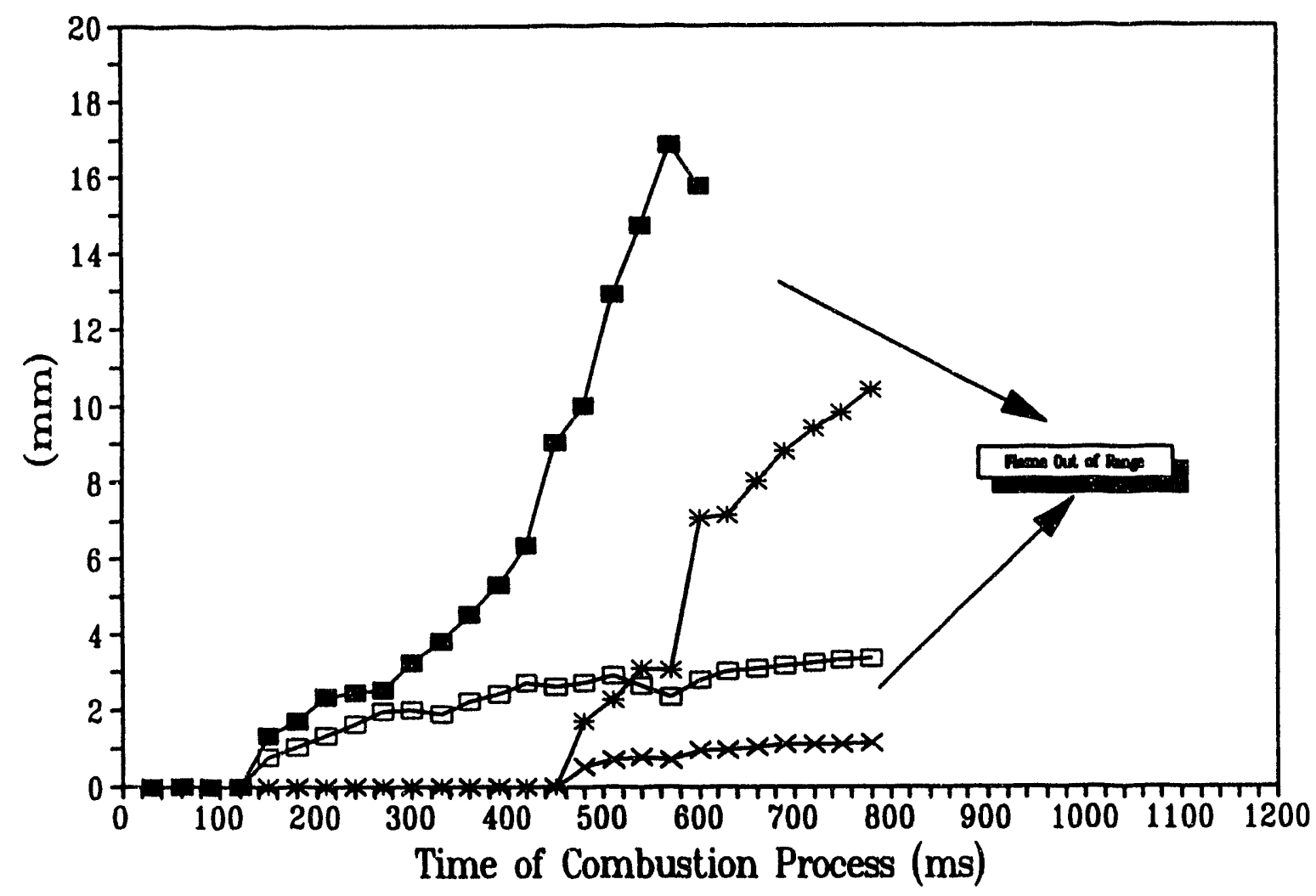

$\rightarrow-$ Flame Height $\quad$ Fame Width $\rightarrow$ Soot Region Height $\rightarrow-$ Soot Region Width

Figure 29. Flame Characteristics of Isolated Medium Volatile Coal Particle 


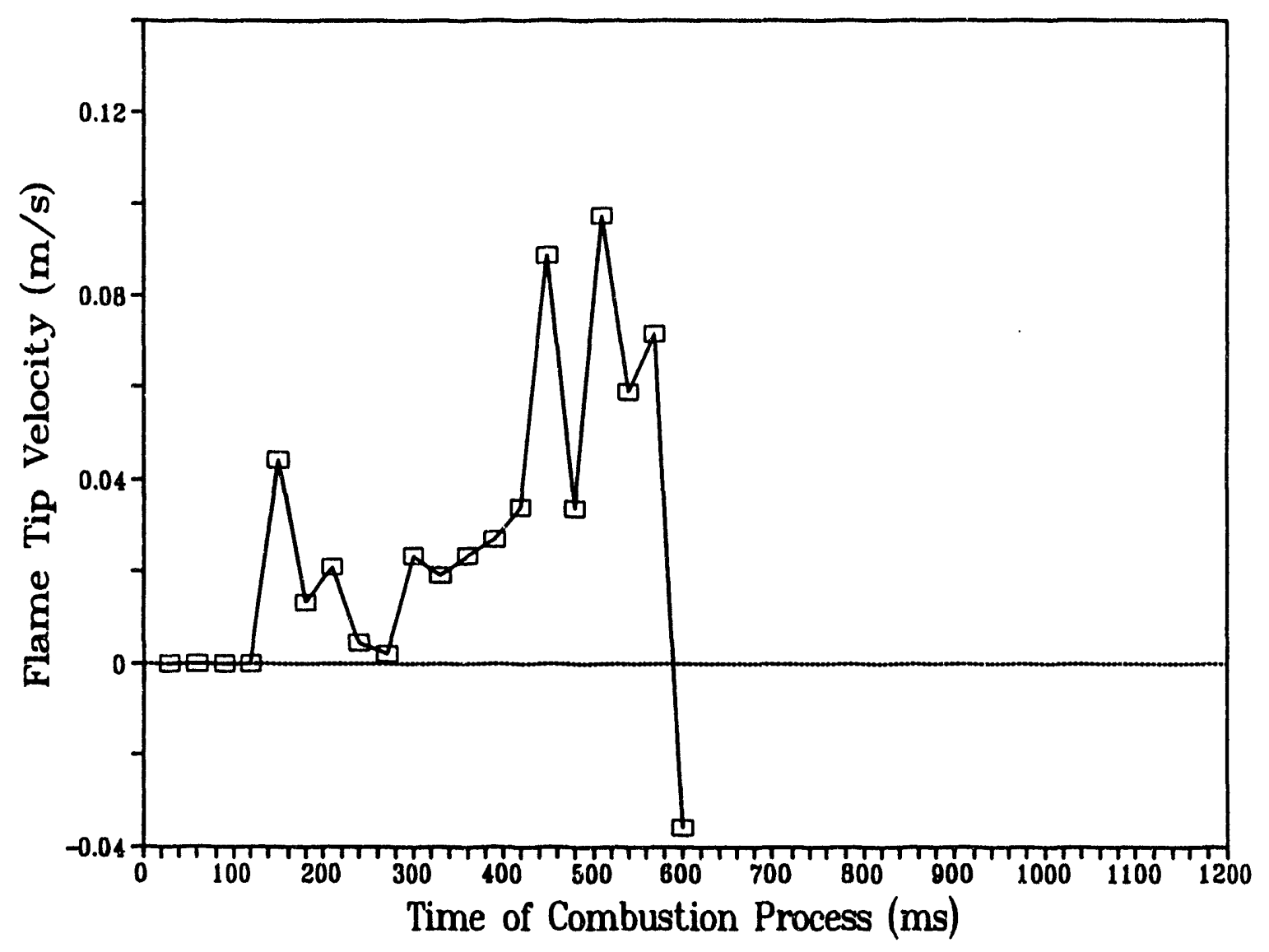

Figure 30. Flame Tip Velocity of Isolated Medium Volatile Coal Particle 
detached from the particle in the early stages of the combustion process and reattached as the process proceeded. The behavior of intermittent ignition was not observed here among the seven experiments conducted on this coal. On the other hand, similar inner black zone was formed at $480 \mathrm{~ms}$ and grew larger as the flame became larger and larger. Combustion time was not measured since the flame after $630 \mathrm{~ms}$ went beyond the monitor's view, in all the seven experiments conducted. This behavior continued for the rest of the recorded combustion period $(1470 \mathrm{~ms})$. Table 5 summarizes the experimental data of the flame characteristics.

\section{VI.3.3 Low Volatile Coal (PSOC 1516)}

The physical and chemical analysis are given in Table 2. A distinguishing feature of this coal is its low volatile content. Due to this reason, the combustion process was observed to be short and ended after $480 \mathrm{~ms}$ only from its exposure time (Figure 31 ). In spite of this major difference from other coals, ignition sequence and flame characteristics (Figure 32) resembled the medium volatile coal to a great extent. Ignition started at the surface of the particle, volatile ejected upwards in the direction of the convective flow ( $\operatorname{Re}=1.48$ and $\mathrm{Nu}=2.62$ ), and burned in the gas phase (homogeneous combustion). Consequently, both processes proceeded in parallel, black inner zone was formed at $120 \mathrm{~ms}$, flame height went beyond the monitor's view at 420 ms, no intermittent ignition was observed among six experiments conducted on this coal, and the particle swelled after the completion of the combustion process by 2.4 times the original size. Comparing Figures 32 and 26 , it is seen that the inner zone stayed over 
Table 5. Flame Characteristics of Medium Volatile Isolated Coal Particle

Particle Diameter $=0.84 \mathrm{~mm}$

Ignition Time $=30 \mathrm{~ms}$

Combustion Time $>11470 \mathrm{~ms}$

\begin{tabular}{|c|c|c|c|c|c|c|}
\hline Trame & 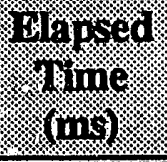 & $\begin{array}{l}\text { Tame: } \\
\text { nevght } \\
\text { (minim) }\end{array}$ & $\begin{array}{l}\text { glane } \\
\text { Wirati } \\
\text { (minim }\end{array}$ & $\begin{array}{l}\text { Inner 2one } \\
\text { geght }\end{array}$ & 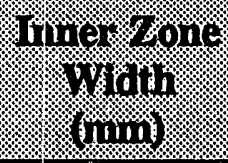 & 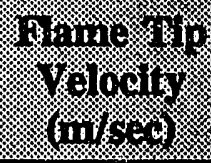 \\
\hline 1 & 30 & - & - & - & - & - \\
\hline 2 & $\$ 50$ & - & - & - & - & - \\
\hline 3 & 90 & - & - & - & - & - \\
\hline 4 & 120 & - & - & - & - & - \\
\hline 5 & 150 & 1.32 & 0.76 & - & - & 0.044 \\
\hline 6 & 180 & 1.71 & 1.01 & - & - & 0.013 \\
\hline 7 & 210 & 2.34 & 1.33 & - & - & 0.021 \\
\hline 8 & 240 & 2.47 & 1.64 & - & - & 0.004 \\
\hline 9 & 270 & 2.53 & 1.96 & - & - & 0.002 \\
\hline 10 & 300 & 3.23 & 2.02 & - & - & 0.023 \\
\hline 11 & 330 & 3.80 & 1.91 & - & - & 0.019 \\
\hline 12 & 360 & 4.50 & 2.21 & - & - & 0.023 \\
\hline 13 & 390 & 5.31 & 2.40 & - & - & 0.027 \\
\hline 14 & 420 & 6.33 & 2.73 & - & - & 0.034 \\
\hline 15 & 450 & 8.99 & 2.59 & - & - & 0.088 \\
\hline 16 & 480 & 10 & 2.72 & 1.71 & 0.51 & 0.033 \\
\hline 17 & 510 & 12.92 & 2.91 & 2.28 & 0.69 & 0.097 \\
\hline 18 & 540 & 14.69 & 2.65 & 3.10 & 0.76 & 0.059 \\
\hline 19 & 570 & 16.84 & 2.34 & 3.04 & 0.70 & 0.072 \\
\hline 20 & 600 & 15.76 & 2.79 & 7.06 & 0.94 & -0.036 \\
\hline
\end{tabular}


Table 5. (Continued)

\begin{tabular}{|c|c|c|c|c|c|c|}
\hline frame & $\begin{array}{l}\text { elapsed } \\
\text { Time- } \\
\text { (ms): }\end{array}$ & $\begin{array}{l}\text { Flame } \\
\text { Height } \\
\text { (mim) }\end{array}$ & $\begin{array}{l}\text { Flame } \\
\text { Width } \\
\text { (mim) }\end{array}$ & $\begin{array}{l}\text { Imner zone } \\
\text { " Ieight }\end{array}$ & $\begin{array}{l}\text { Inner Zone } \\
\text { " Wiath }\end{array}$ & $\begin{array}{l}\text { glame Tip } \\
\text { Velocity } \\
(\mathrm{m} / \mathrm{sec})\end{array}$ \\
\hline 21 & 630 & NA & 3.03 & 7.12 & 0.95 & NA \\
\hline 22 & 660 & $\mathrm{Na}$ & 3.10 & 8 & 1.02 & NA \\
\hline 23 & 690 & NA & 3.18 & 8.80 & 1.09 & NA \\
\hline 24 & 720 & NA & 3.25 & 9.40 & 1.10 & NA \\
\hline 25 & 750 & NA & 3.30 & 9.80 & 1.12 & NA \\
\hline 26 & 780 & NA & 3.36 & 10.38 & 1.14 & NA \\
\hline $\begin{array}{c}27 \\
b \\
49\end{array}$ & 810 & NA & NA & NA & NA & NA \\
\hline
\end{tabular}




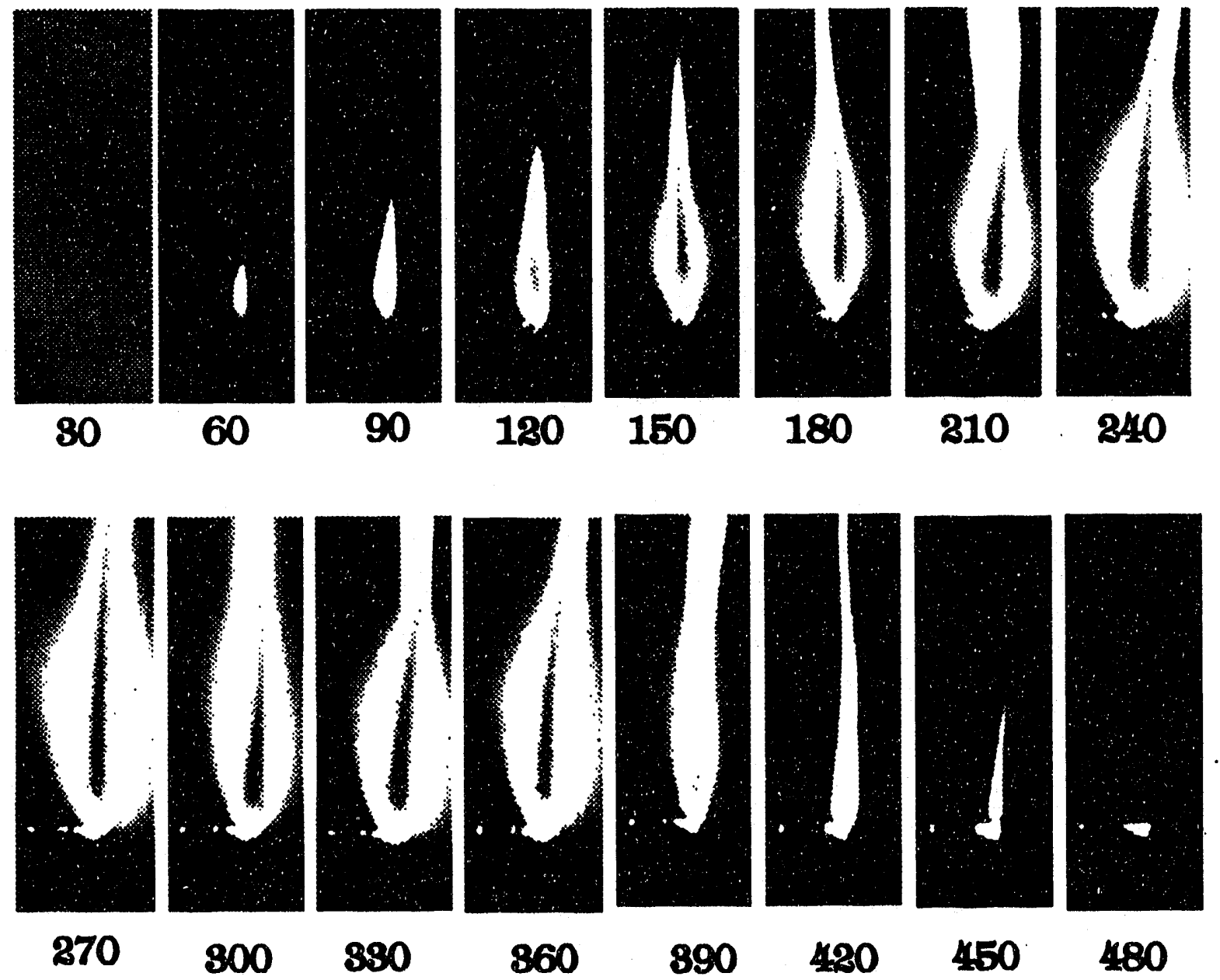

Figure 31a. Ignition and Combustion Sequence for Isolated Low Volatile Coal Particle 

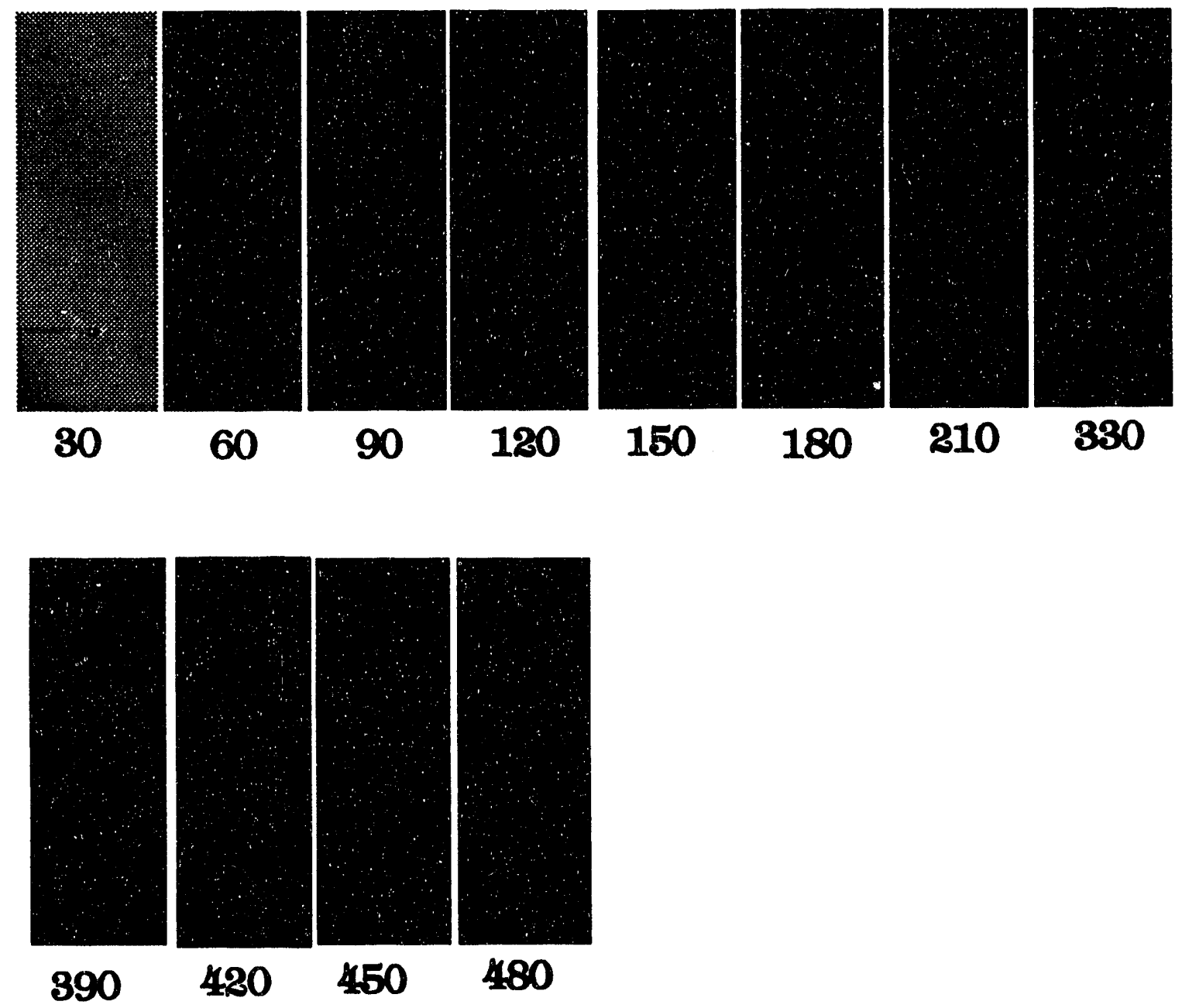

Figure 31b. Images of Ignition and Combustion Sequence with Boundary Detection Function for Isolated Low Volatile Coal Particle 


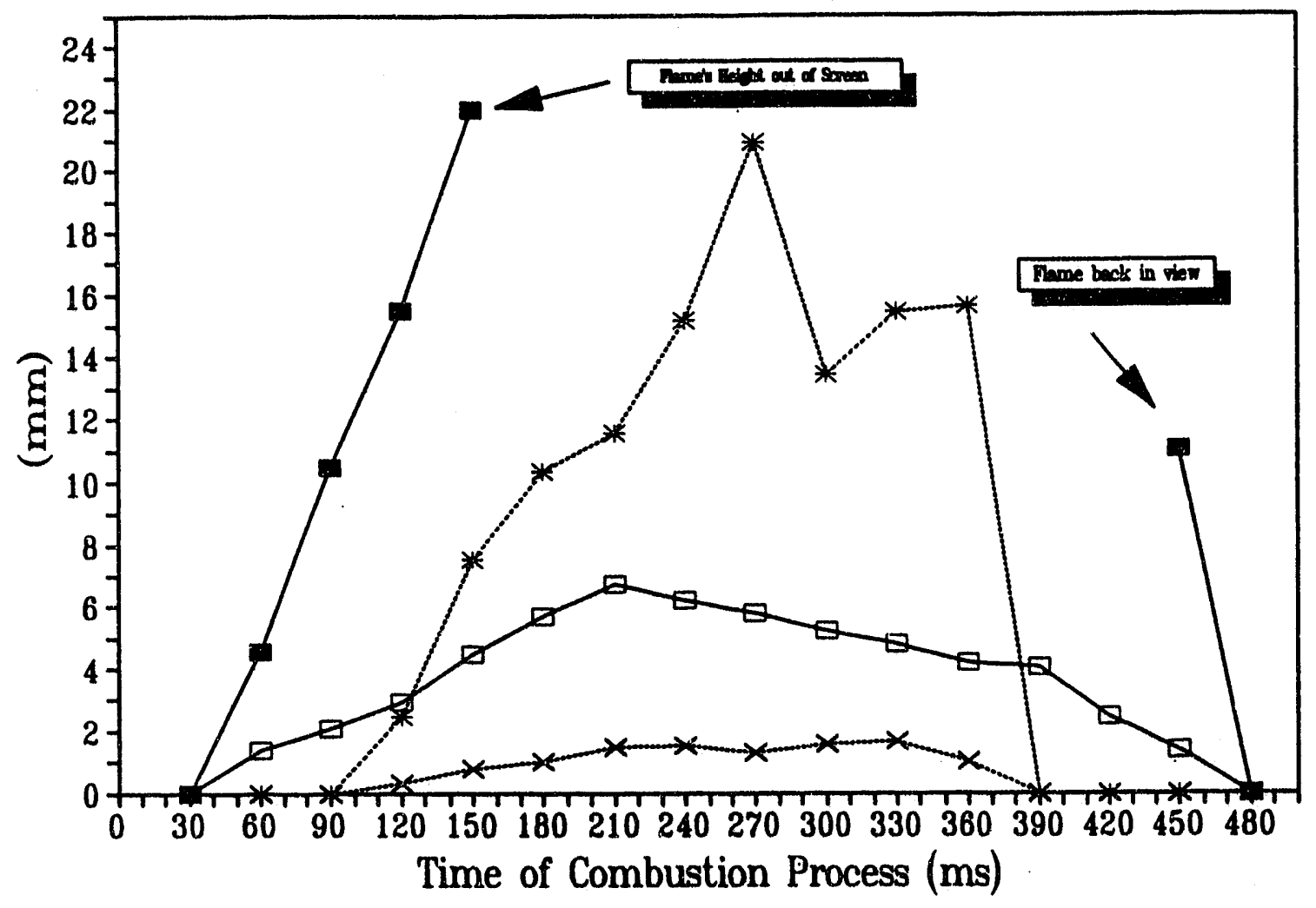

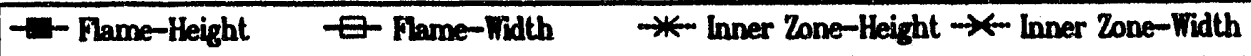

Figure 32. Flame Characteristics of Isolated Low Volatile Coal Particle 
a longer period of time for a high volatile coal particle than for a low volatile coal particle, indicated that reduced volatile matter resulted in a smaller and short lived inner zone. Figure 33 shows the flame tip velocity as a function of time. Table 6 summarizes the experimental data of the flame characteristics.

Figures 34 through 37 show comparison of the three coal types: flame height, flame width, flame tip velocity and ignition, burning and combustion times.

\section{VI.4 Isolated Char Particles}

Upon introducing the char particle on top of the burner $(\operatorname{Re}=1.96$ and $\mathrm{Nu}=$ 2.72), ignition occurred at $270 \mathrm{~ms}$ and started at the surface of the particle (heterogeneous ignition) and more specifically at the leading edge. Unlike the previous coal particles, very little volatile ejected from the particle, yet were not sufficient to form any flame. The particle continued to burn on the surface. As more time elapsed, more heat was supplied to the particle from the vitiated gases causing larger portions of the particle to be combusted heterogeneously. This behavior proceeded until the whole particle was combusted at $510 \mathrm{~ms}$. Figure 38 shows the sequence of the combustion process where the variation of the diameter with time is obvious. This behavior is attributed to the fact that char differs fundamentally from coal. Fixed carbon is the dominant element in char with very little existing volatile. On the other hand, coal typically contains fixed carbon and a large portion of volatile. It is obvious that volatile are the main source for the observed wake flame in previous coal particles, while the 


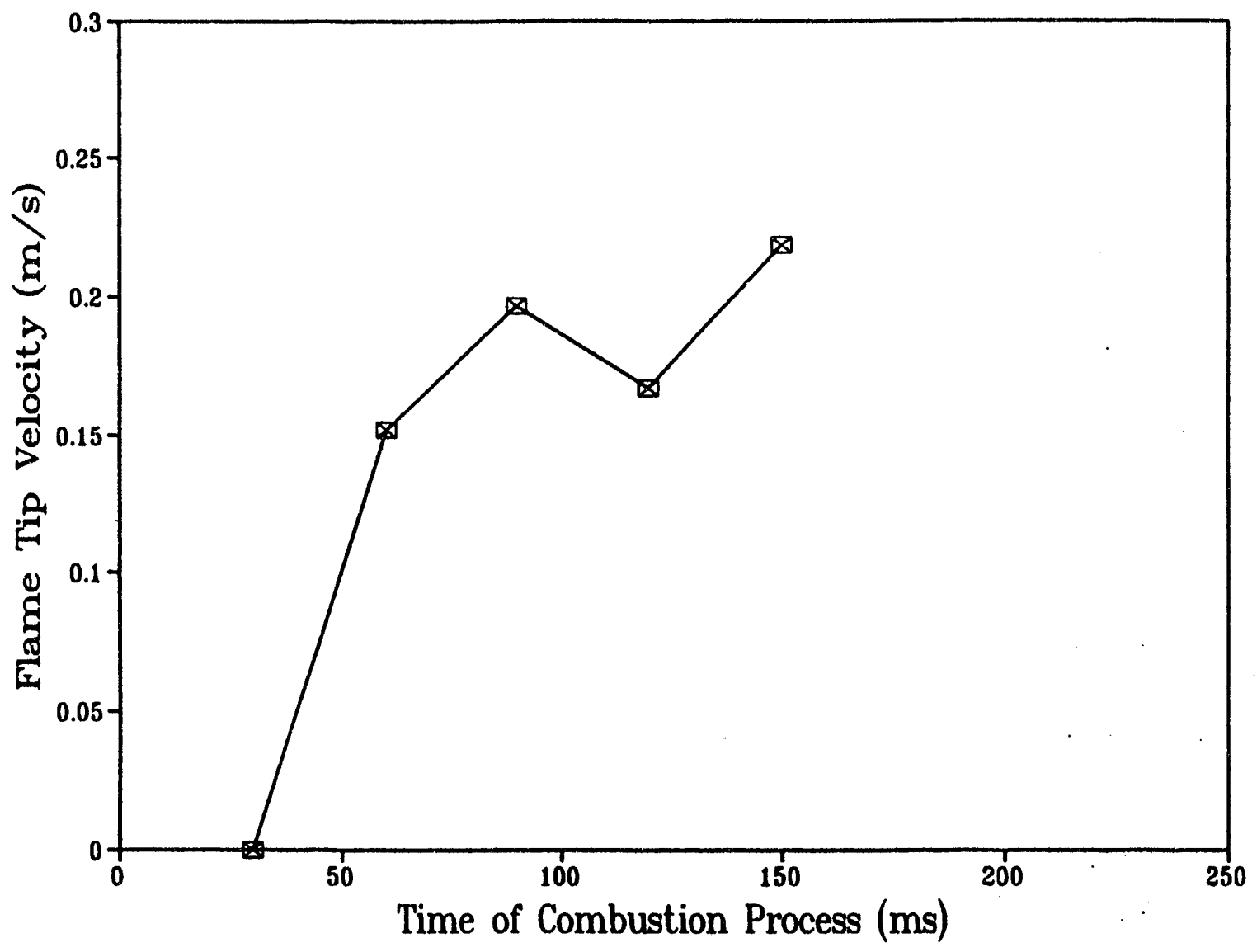

Figure 33. Flame Tip Velocity of Isolated Low Volatile Coal Particle 
Table 6. Flame Characteristics of Low Volatile Isolated Coal Particle

Particle Diameter $=0.81 \mathrm{~mm}$

Ignition Time $=60 \mathrm{~ms}$

Combustion Time $=480 \mathrm{~ms}$

\begin{tabular}{|c|c|c|c|c|c|c|}
\hline Frame: & $\begin{array}{l}\text { Elapsed } \\
\text { Time } \\
\text { (ms) }\end{array}$ & $\begin{array}{l}\text { Tlame } \\
\text { Height } \\
\text { (mimit) }\end{array}$ & $\begin{array}{l}\text { Mlame } \\
\text { Width } \\
\text { (mm) }\end{array}$ & $\begin{array}{l}\text { Immer Lone } \\
\text { Height" } \\
(\mathbf{m m})\end{array}$ & $\begin{array}{l}\text { Inner } 2 \text { cones } \\
\text { Widat }\end{array}$ & 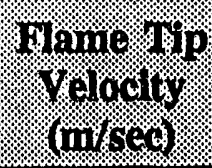 \\
\hline 1 & 30 & - & - & - & - & - \\
\hline 2 & 60 & 4.56 & 1.39 & - & - & 0.152 \\
\hline 3 & 90 & 10.45 & 2.09 & - & - & 0.196 \\
\hline 4 & 120 & 15.45 & 2.91 & 2.46 & 0.32 & 0.167 \\
\hline 5 & 150 & 22 & 4.49 & 7.53 & 0.76 & 0.218 \\
\hline 6 & 180 & $\mathrm{NA}^{\dagger}$ & 5.70 & 10.32 & 1.01 & NA \\
\hline 7 & 210 & NA & 6.71 & 11.52 & 1.46 & NA \\
\hline 8 & 240 & NA & NA & 15.13 & 1.52 & NA \\
\hline 9 & 270 & NA & NA & 20.89 & 1.27 & NA \\
\hline 10 & 300 & $\mathrm{NA}$ & NA & 13.42 & 1.58 & NA \\
\hline 11 & 330 & NA & NA & 15.44 & 1.65 & NA \\
\hline 12 & 360 & NA & NA & 15.63 & 1.01 & NA \\
\hline 13 & 390 & NA & 4.05 & 0 & 0 & NA \\
\hline 14 & 420 & NA & 2.47 & 0 & 0 & NA \\
\hline 15 & 450 & 11.02 & 1.39 & 0 & 0 & NA \\
\hline 16 & 480 & 0 & 2.72 & 0 & 0 & NA \\
\hline
\end{tabular}




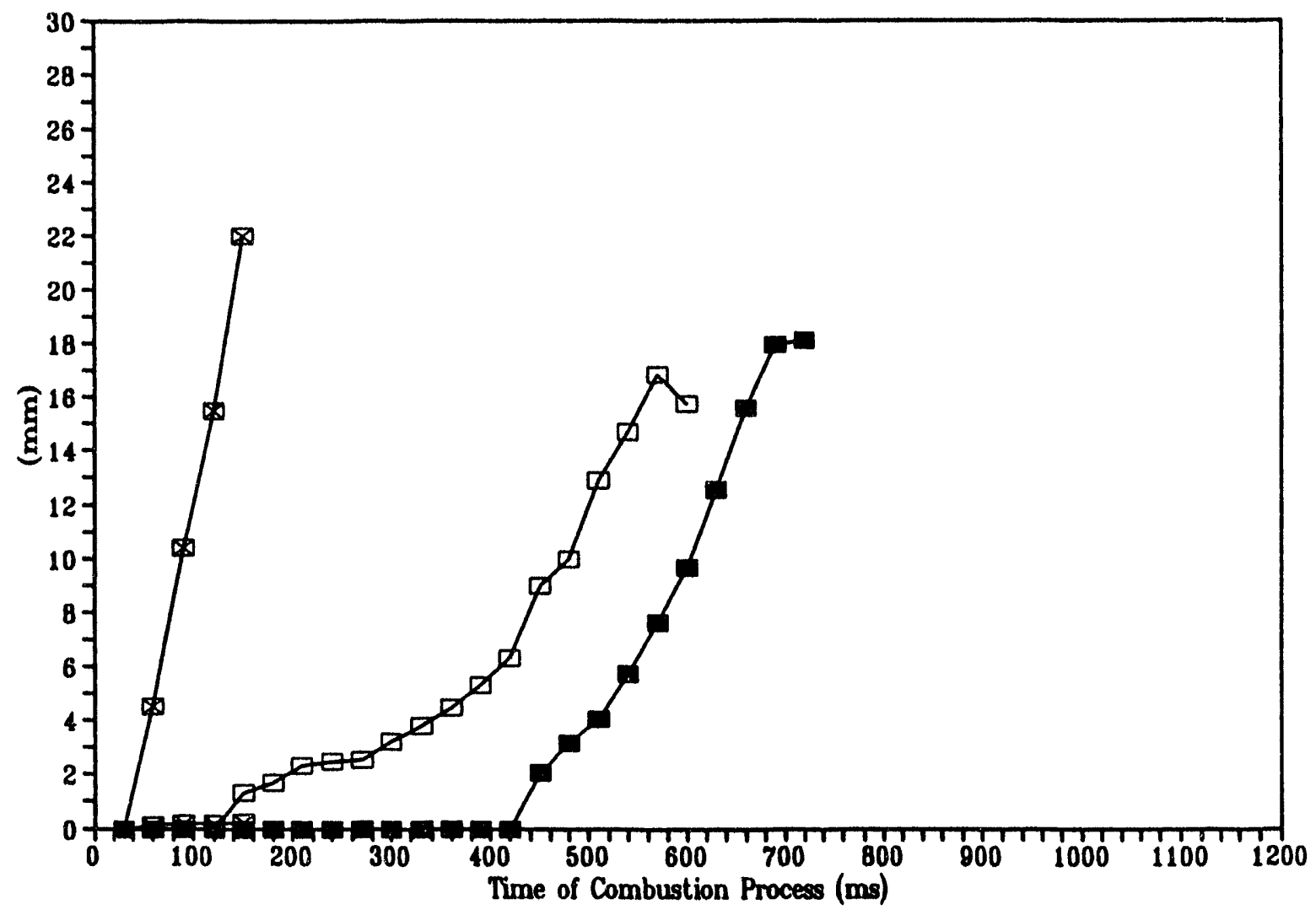

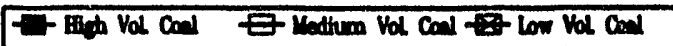

Figure 34. Comparison of Flame Height for Isolated Coal Particles 


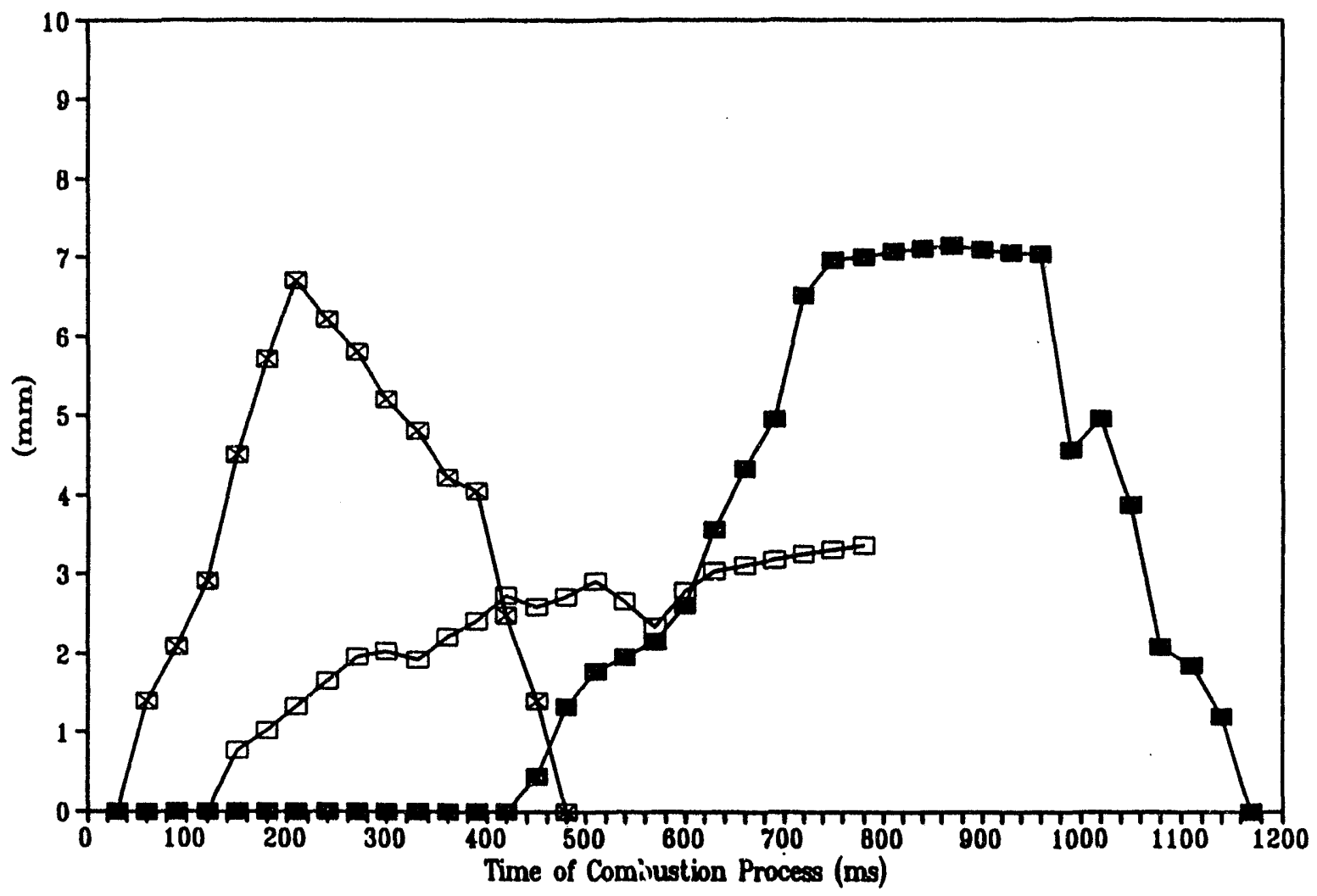

$\rightarrow-$ High Vol Coal $\square$ Medium Vol Coal - Lor Vol Coal

Figure 35. Comparison of Flame Width for Isolated Coal Particles 


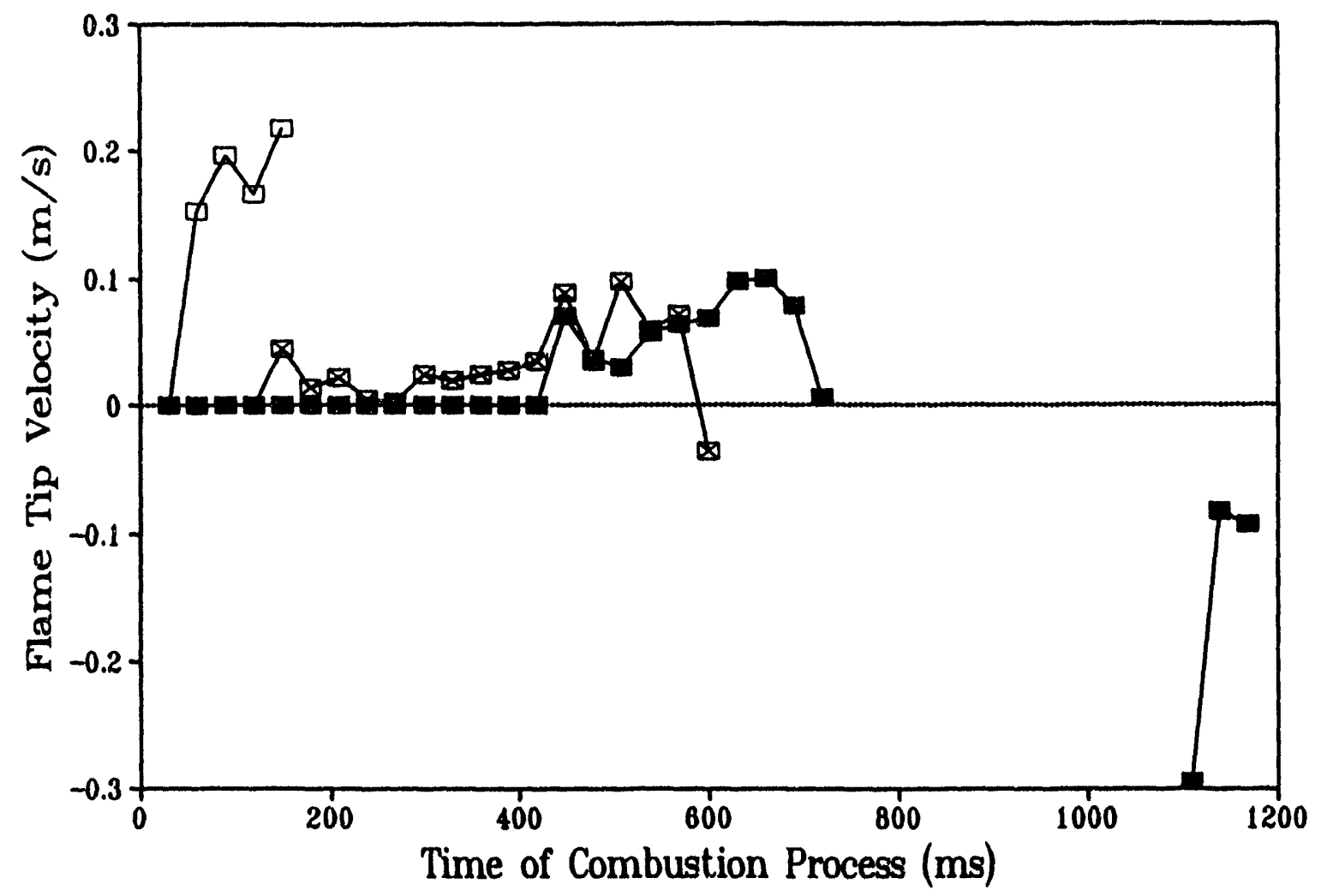

High Vol Coal $\rightarrow$-Medium Vol Coal $\square$ Low Vol Coal

Figure 36. Comparison of Flame Tip Velocity for Isolated Coal Particles 


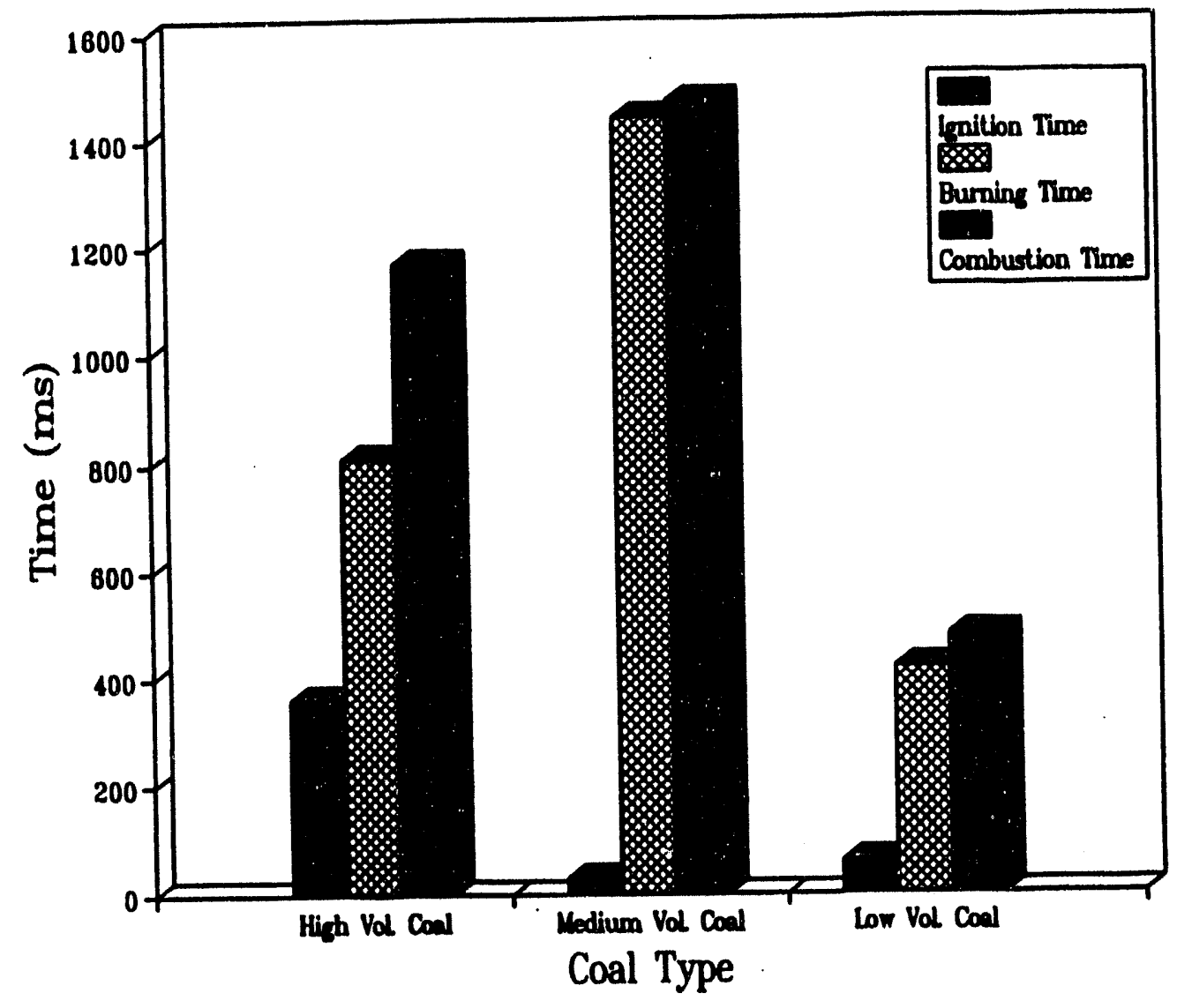

Figure 37. Comparison of Ignition, Burning, and Combustion Times for Isolated Coal Particles 

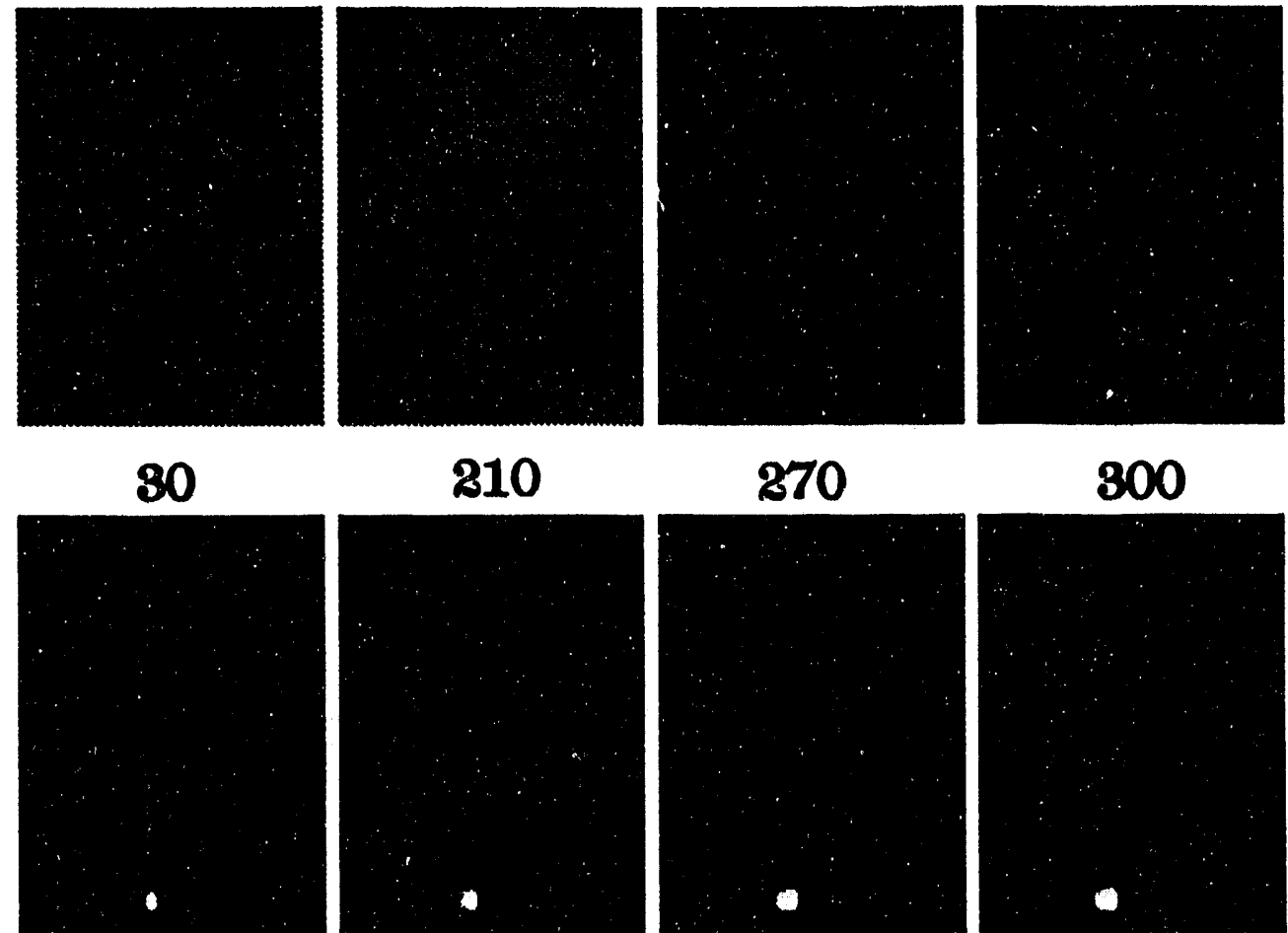

210

$\mathbf{8 7 0}$

300
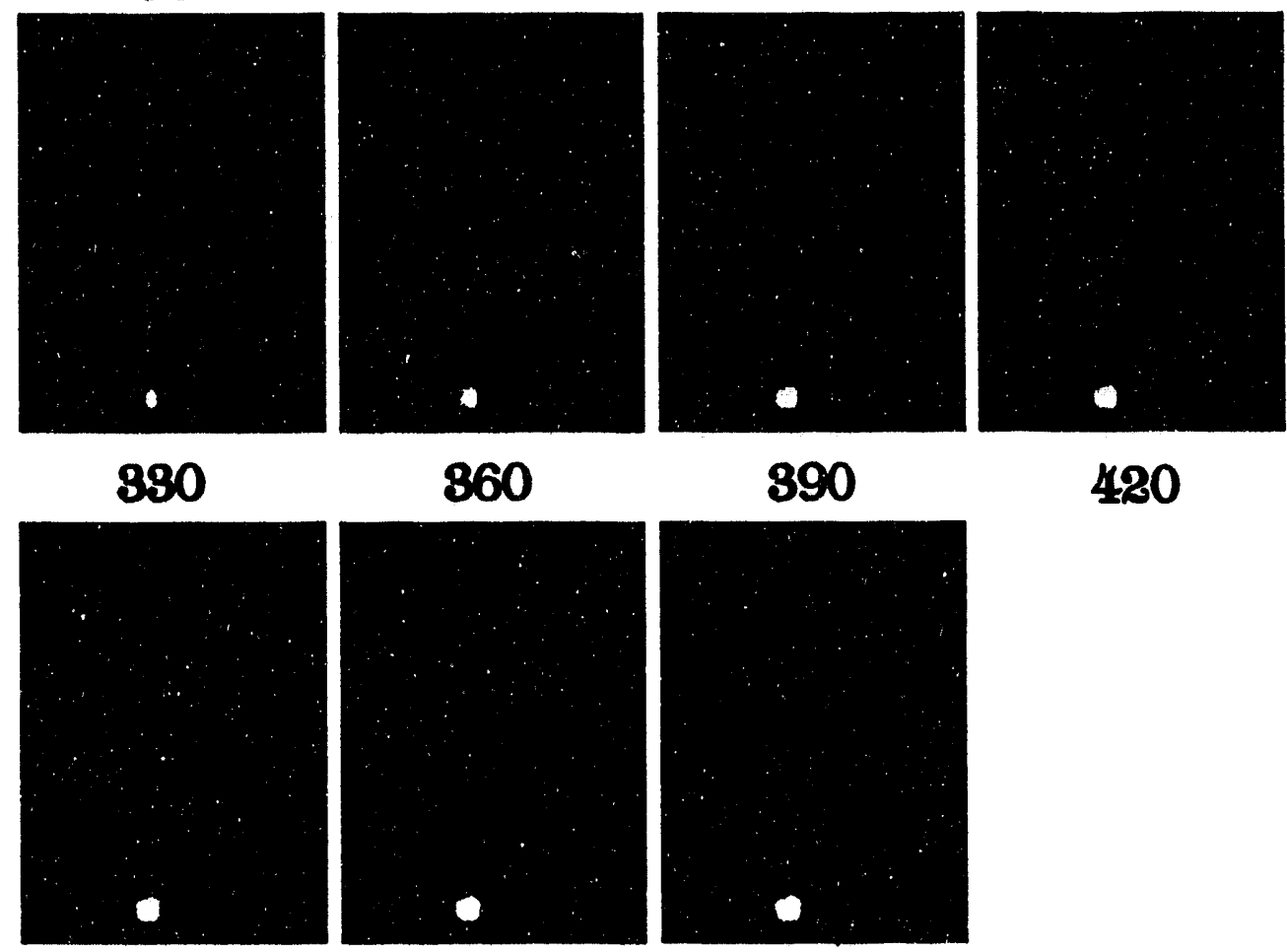

860

390

480

450

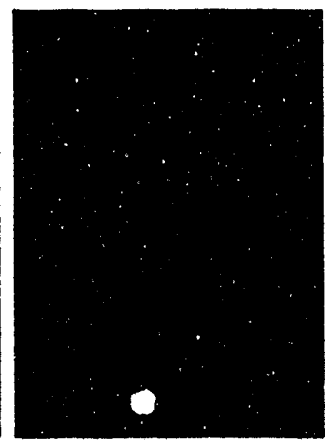

480

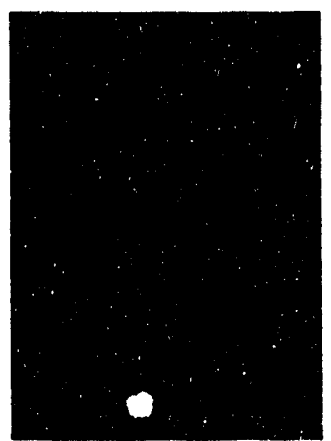

510

Figure 38a. Ignition and Combustion Sequence for Isolated Char Particle 


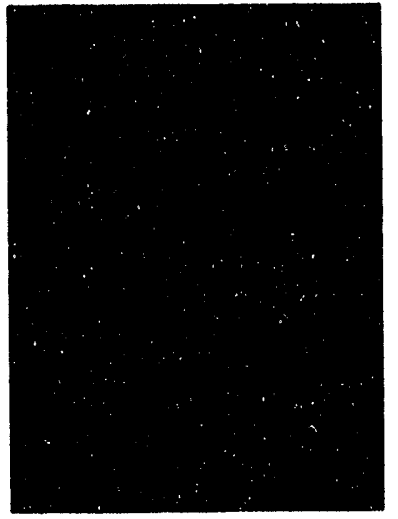

270

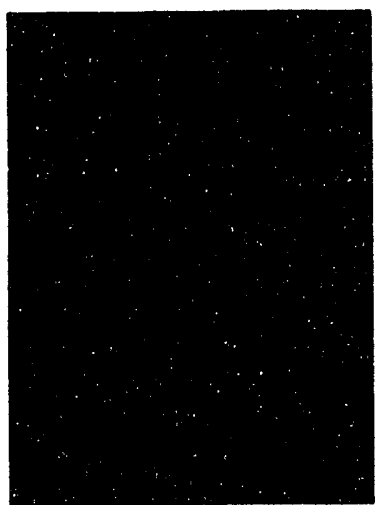

360

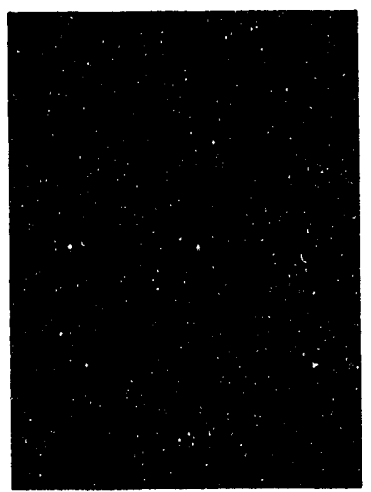

450

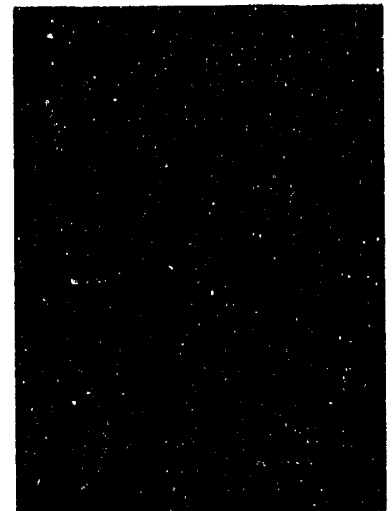

290

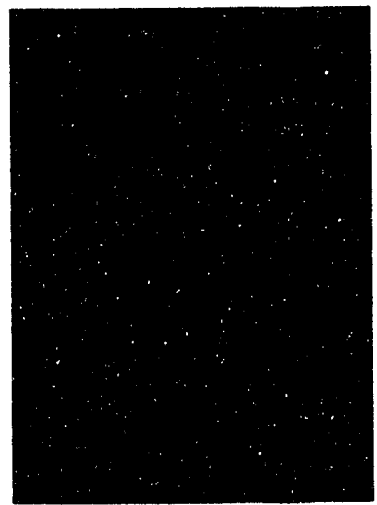

390

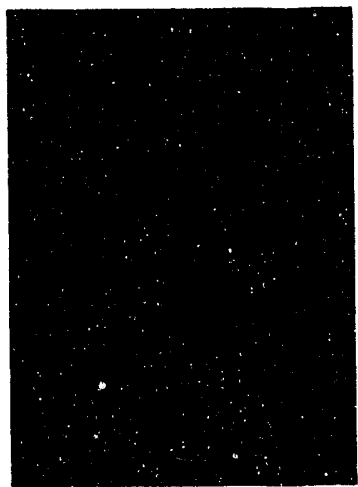

480

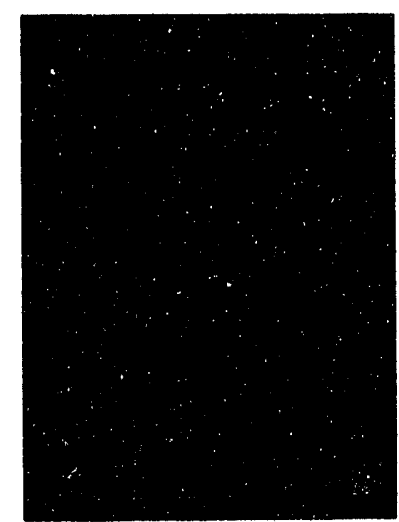

330

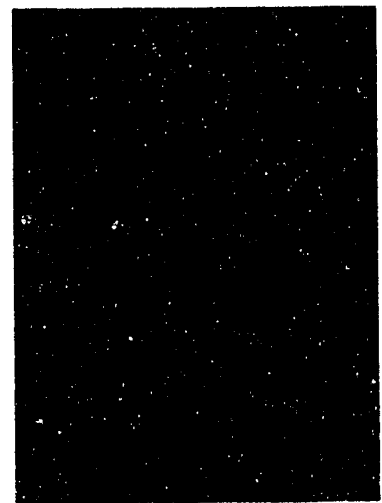

420

Figure 38b. Images of Ignition and Combustion Sequence With Boundary Detection Function for Isolated Char Particle 
combustion behavior in the char particle is simply the behavior of the fixed carbon upon burning. Examining the particle after combustion, it consisted mainly of ash.

\section{5 Coal Two-Particle Arrays}

\section{VI.5.1 Hirh Volatile Coal (PSOC 1447)}

\section{VI.5.1.1 Horizontal Geometry}

In this experiment, two similar particles were placed horizontally apart from each other and were allowed to burn simultaneously upon exposing them to the hot environment provided by the flat flame burner. The distance between the two particles centers, $l_{y}$ was varied from $2.2 \mathrm{~mm}$ to $15.88 \mathrm{~mm}$, to explore its effect on the ignition and combustion characteristics of the two-particle arrays. Also, three different particle sizes $(1.0,0.8,0.6 \mathrm{~mm})$ were employed here (Table 7). The effect of particles sizes was investigated as weli.

As previously observed with the isolated particles, ignition started at the particles surfaces. At a very close distance $(2.2 \mathrm{~mm})$ where the two particles were expected to behave as a single particle, the ignition for large particle array $\left(d_{p}=1.0 \mathrm{~mm}\right)$ and intermediate particle array $\left(d_{p}=0.8 \mathrm{~mm}\right)$ started at $150 \mathrm{~ms}$ and $210 \mathrm{~ms}$ respectively, while at $305 \mathrm{~ms}$ for the small particle array $\left(d_{p}=0.6 \mathrm{~mm}\right)$. This is possibly due to a higher heat loss in the case of the smaller particles. A flame was formed for each particle due to the burning of the ejected volatile. Merging of flames occurred almost immediately and a common flame was formed. Figure 39 shows a typical sketch of 
Table 7. Base Case and Parametric Conditions for Char/Coal Two-Particle Arrays

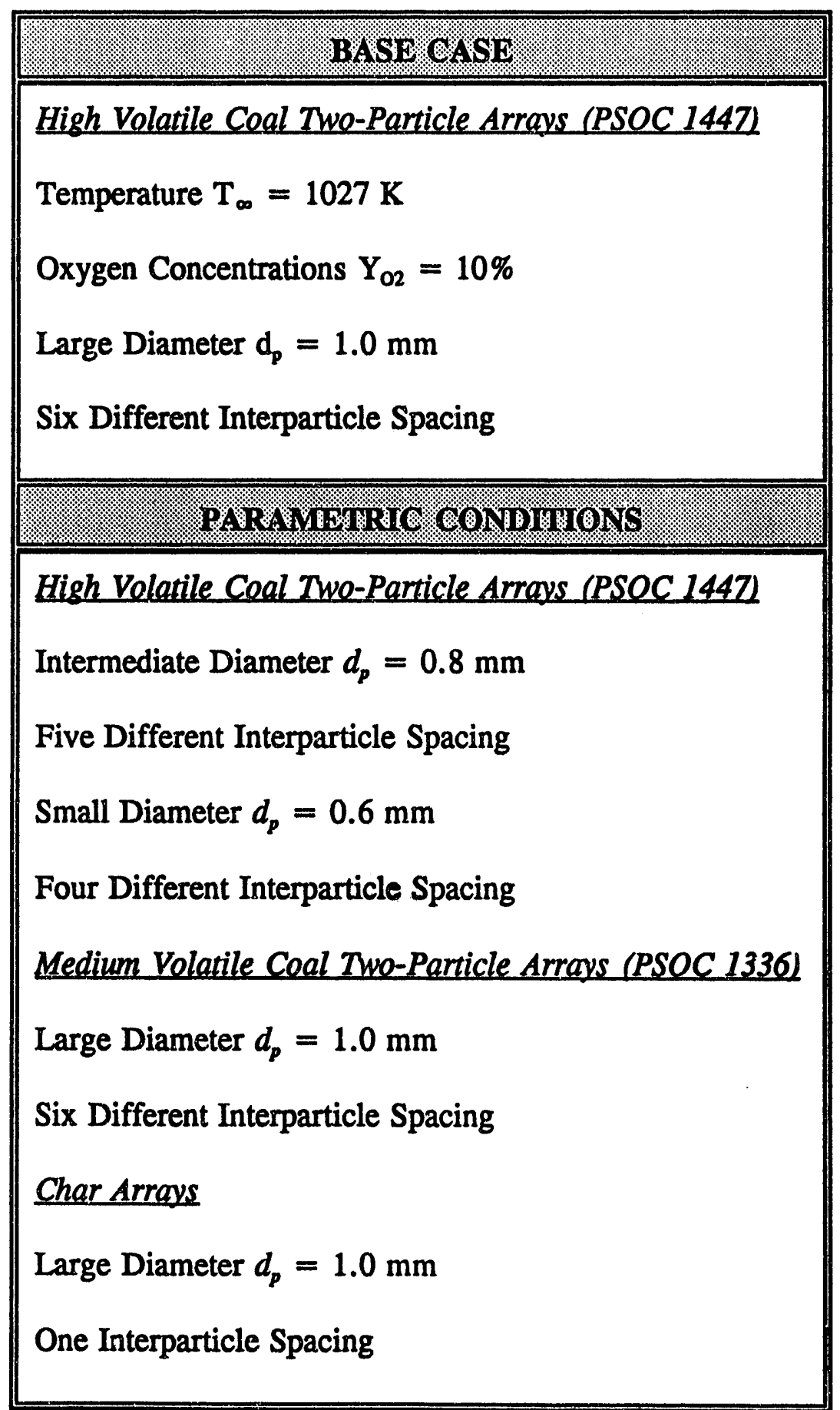




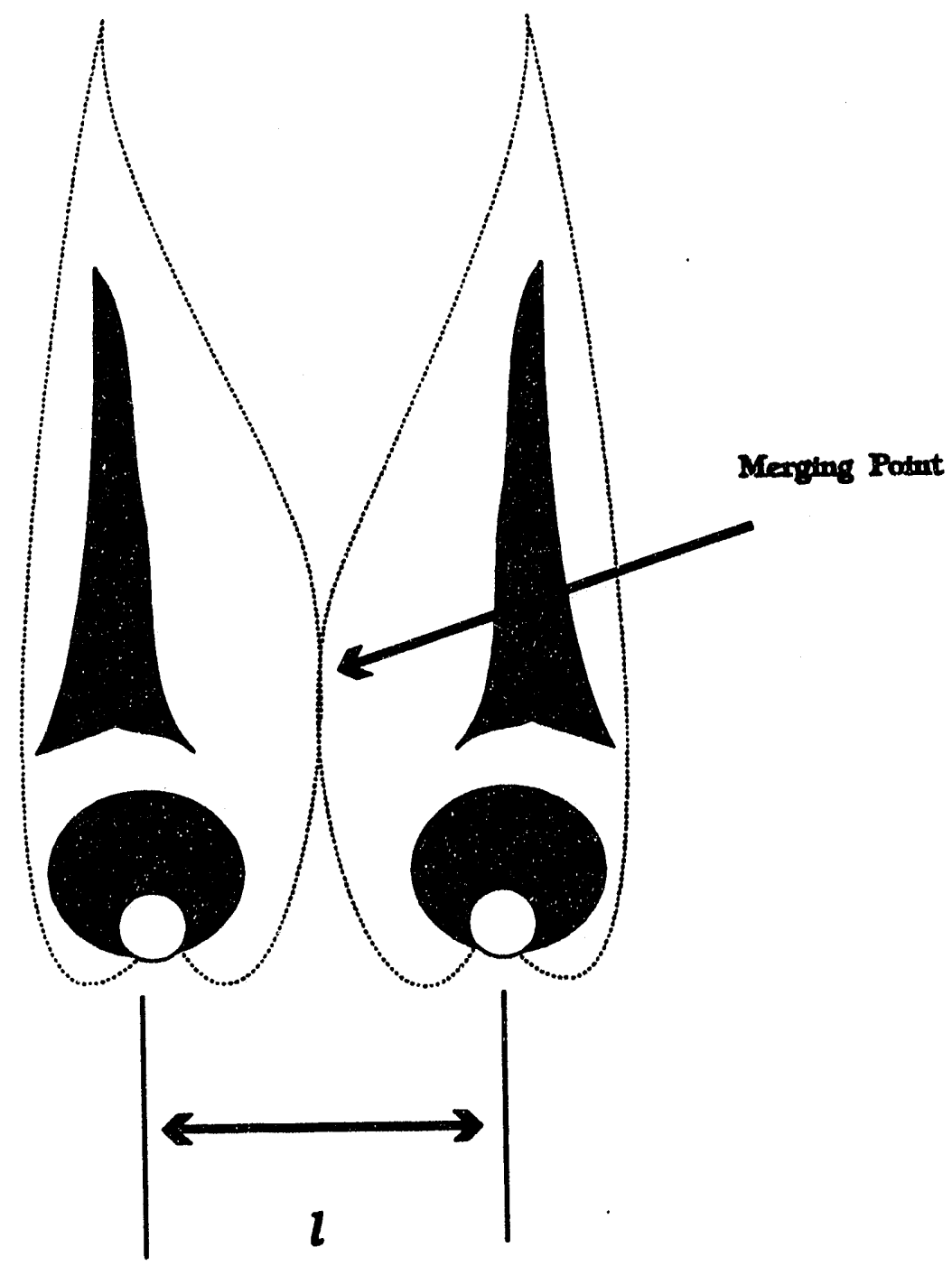

Diatance Between Particien Centens

Figure 39. A Typical Sketch of Combustion of Two-Particle Array in Vitiated Environment 
combustion of two-particle array in vitiated environment. It is worth mentioning that for a given array, the merging time increased as the distance between the particles increased. However, beyond a certain distance (critical distance), flames did not merge and each particle had its own flame. Figures 40 through 42 show this behavior for the three arrays. At the critical distance, the particles were expected to behave as if they were isolated. Yet, no intermittent ignition was observed throughout the combustion process. Thus, it is believed that at farther distances, even if the particles flames did not merge, or even if there is no interaction due to oxygen deficiency, there is still an interactive effect between these particles. It is hypothesized that there is a strong radiation interaction between sooty flame and the particles. Each particle is expected to absorb some radiation energy from the other. Figures 43 through 57 show the ignition and combustion sequences for these arrays. The combustion time was not obtained since the combustion process duration exceeded the number of captured frames for the process.

Additionally, the direction of the flame was drifted to the left or to the right depending on the shape of the particles. Normally, the flow from the burner is located at the center and in the case of an isolated particle it directs the flame to propagate straight upwards. However, in the case of two particles, once the flow passes between the particles, the shape and direction of their edges decide on the drift direction. Moreover, a black inner zone was observed as in the case of isolated particles.

Comparing the three particle sizes (Figure 58), many observations deserve 


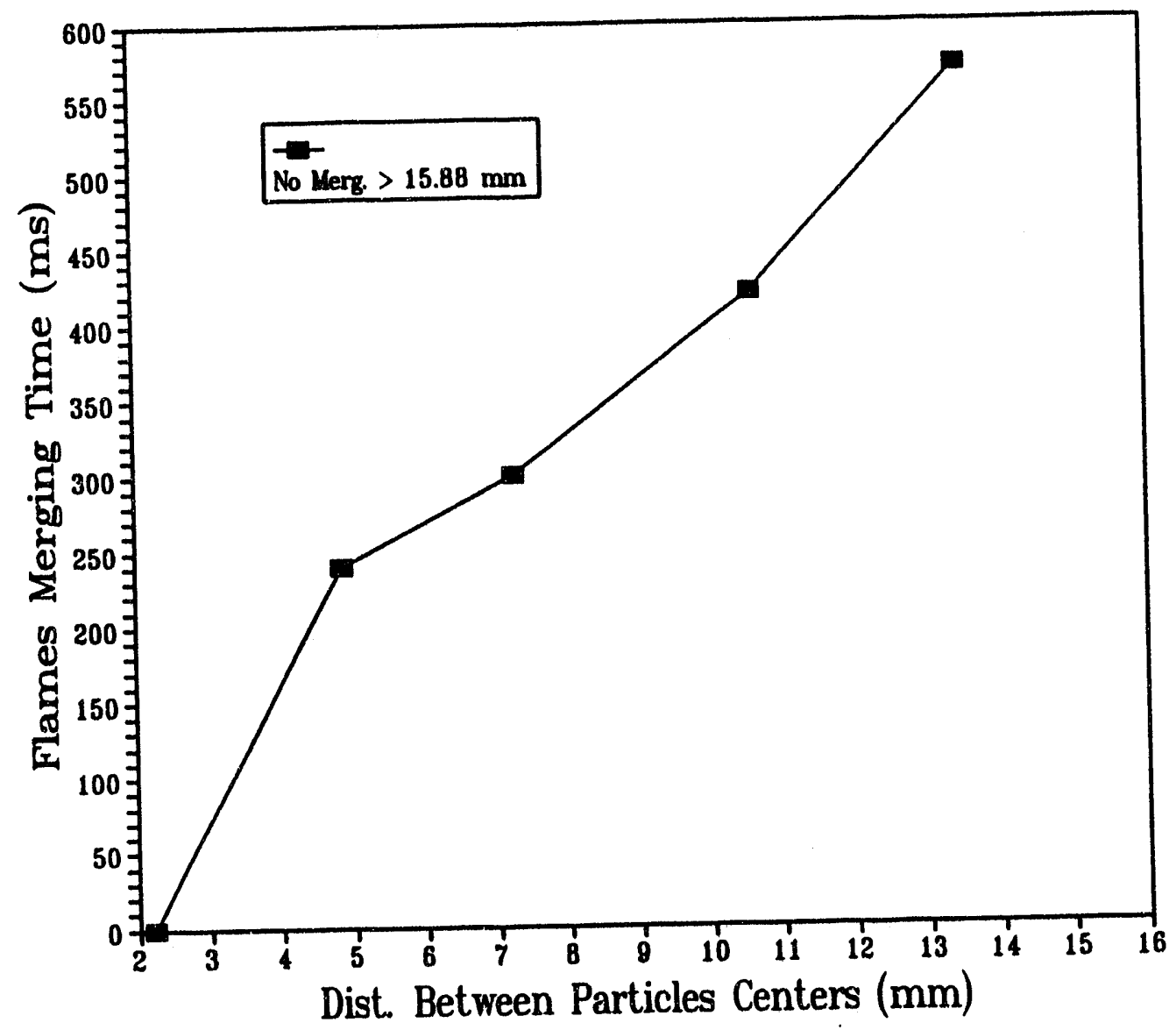

Figure 40. Variation of Flames Merging Time with Interparticle Spacing for Large High Volatile Coal Two-Particle Array 


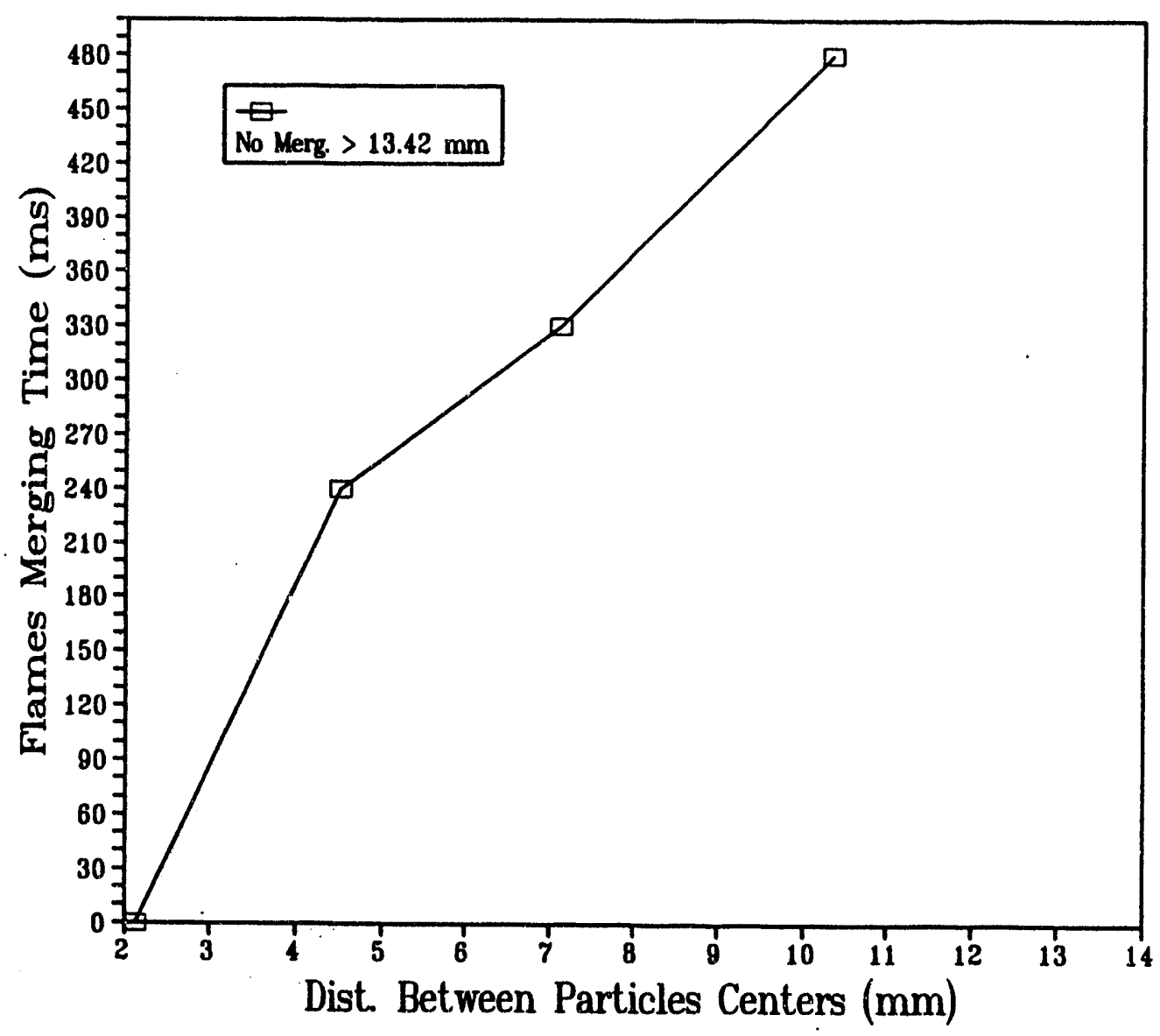

Figure 41. Variation of Flames Merging Time with Interparticle Spacing for Intermediate High Volatile Coal Two-Particle Array 


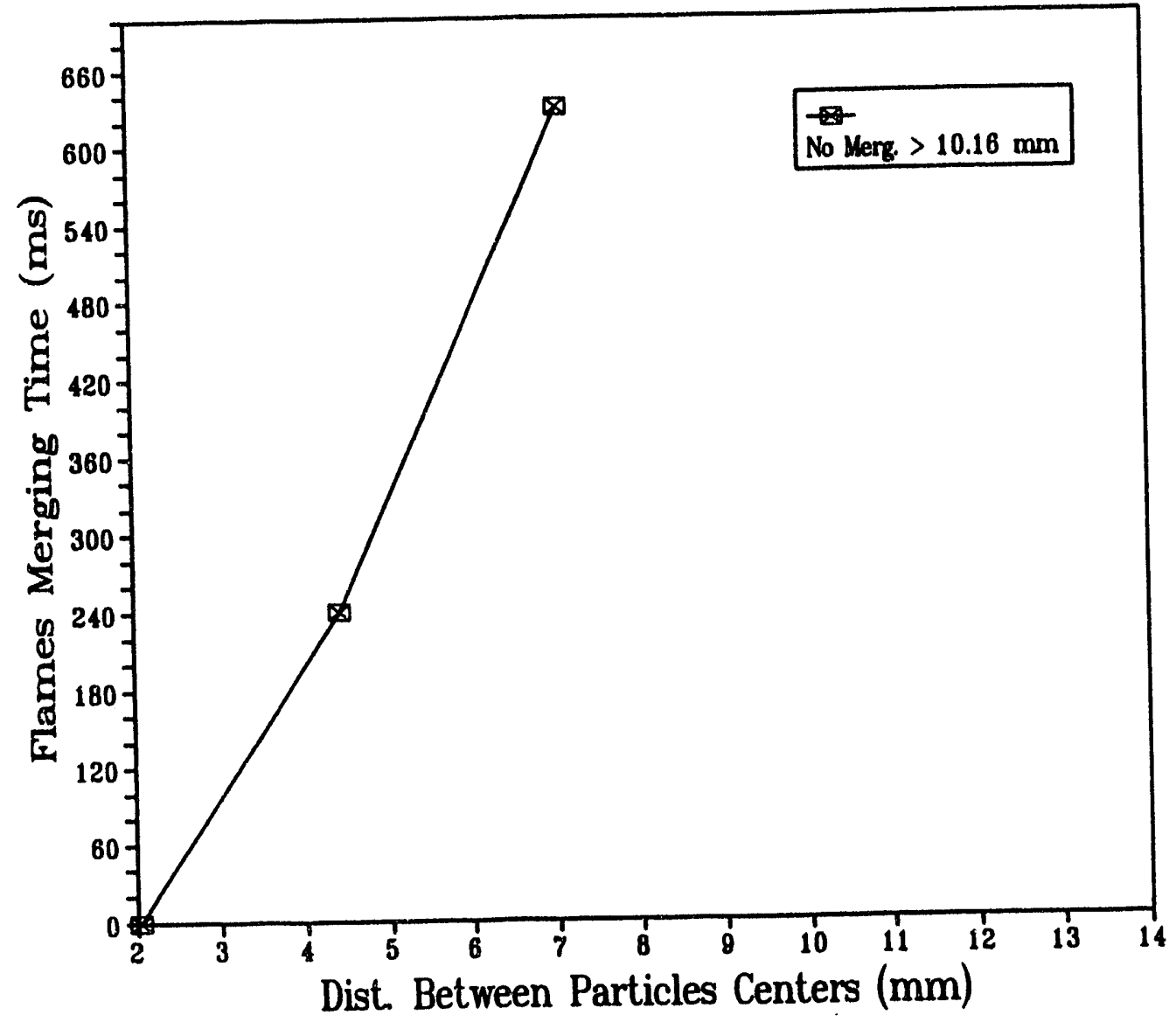

Figure 42. Variation of Flames Merging Time with Interparticle Spacing for Small High Volatile Coal Two-Particle Array 


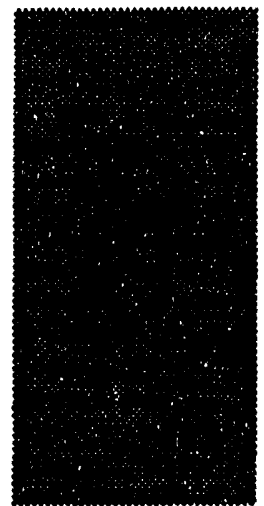

30

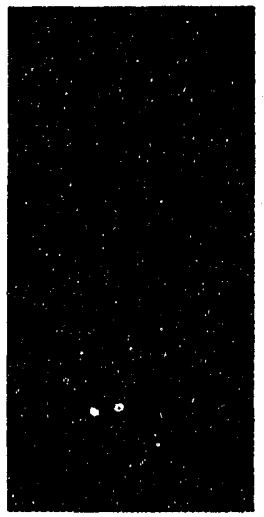

330

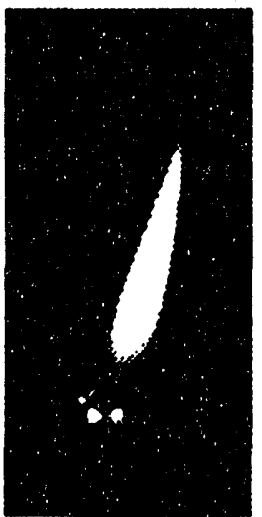

660

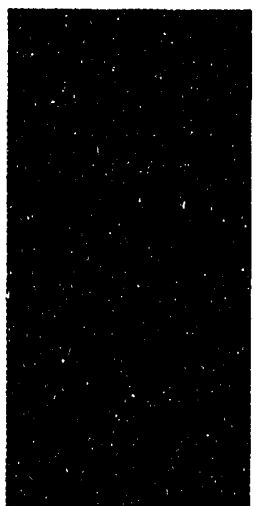

210

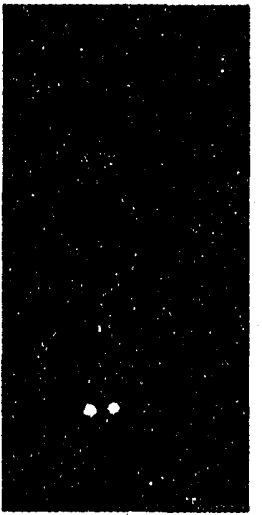

420

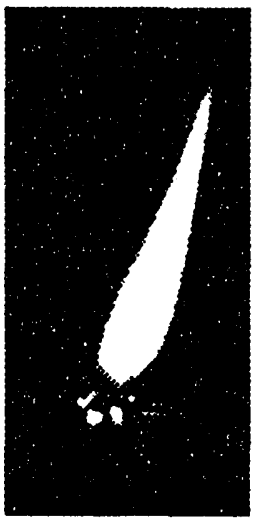

720

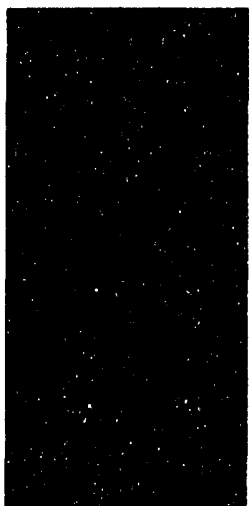

240

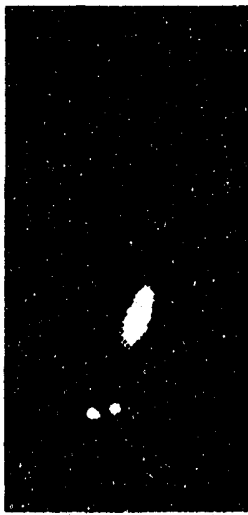

600

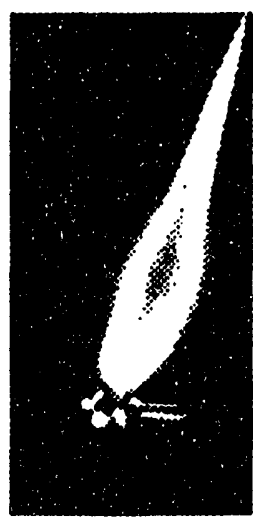

810

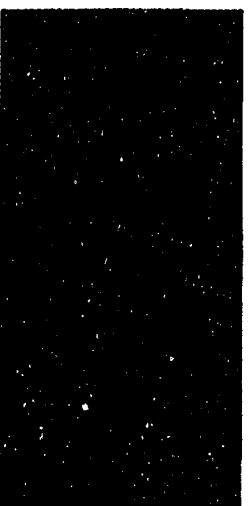

270

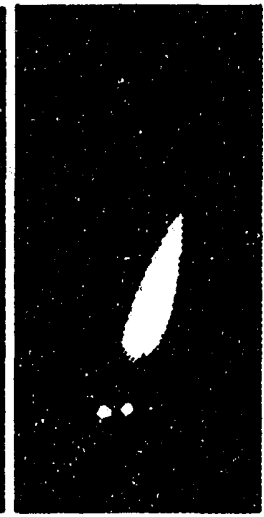

630

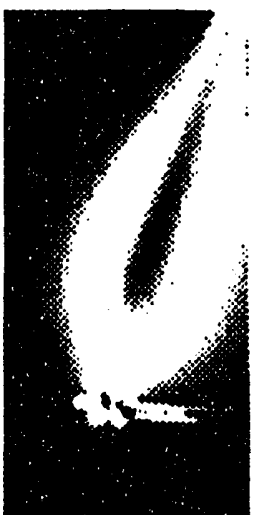

930

Figure 43a. Ignition and Combustion Sequence for Large High Volatile Coal Two-Particle Array $(l=2.2 \mathrm{~mm})$, Numbers are in $\mathrm{msec}$ 

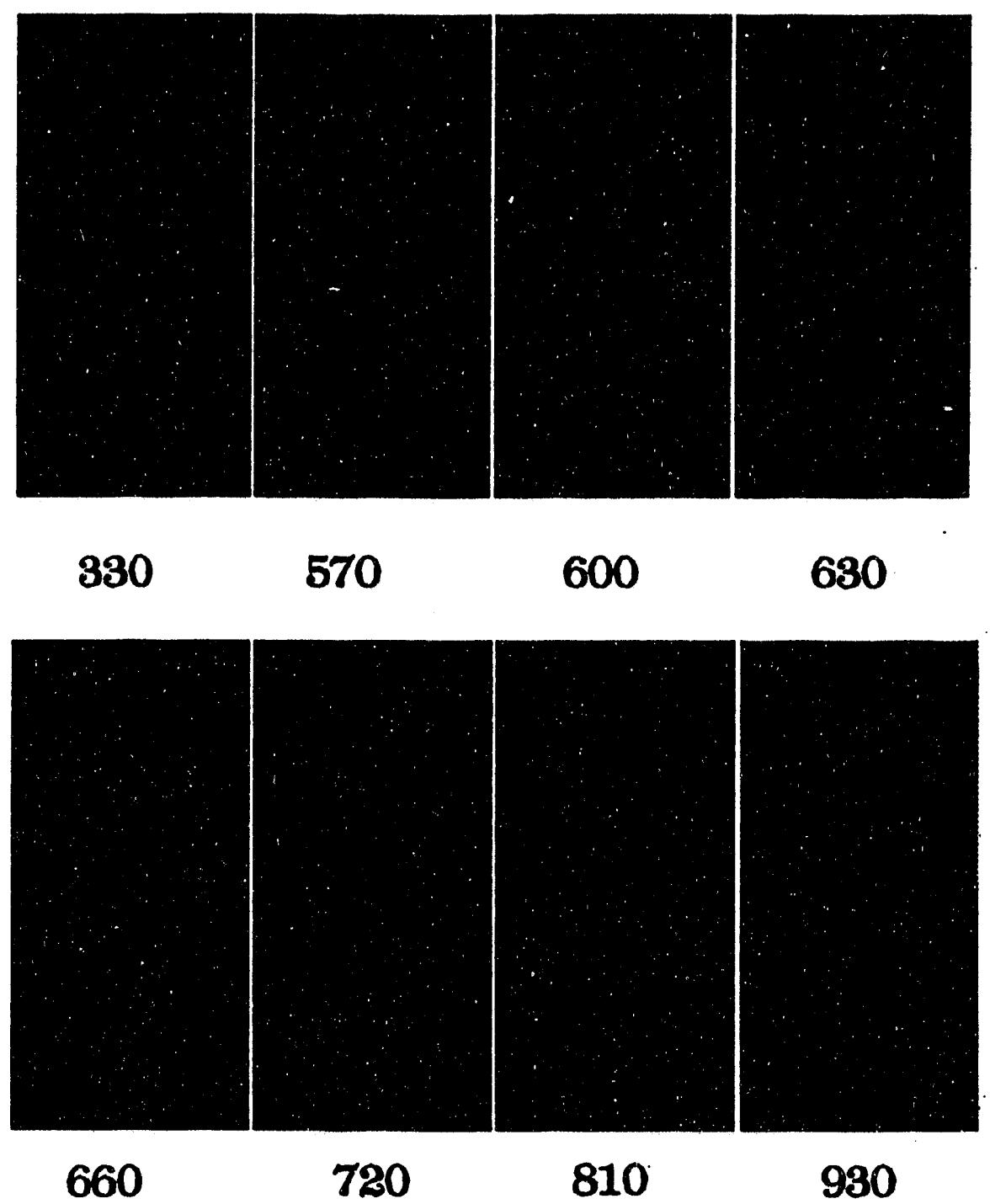

Figure 43b. Images of Ignition and Combustion Sequence With Boundary

Detection Function $(l=2.2 \mathrm{~mm})$ 

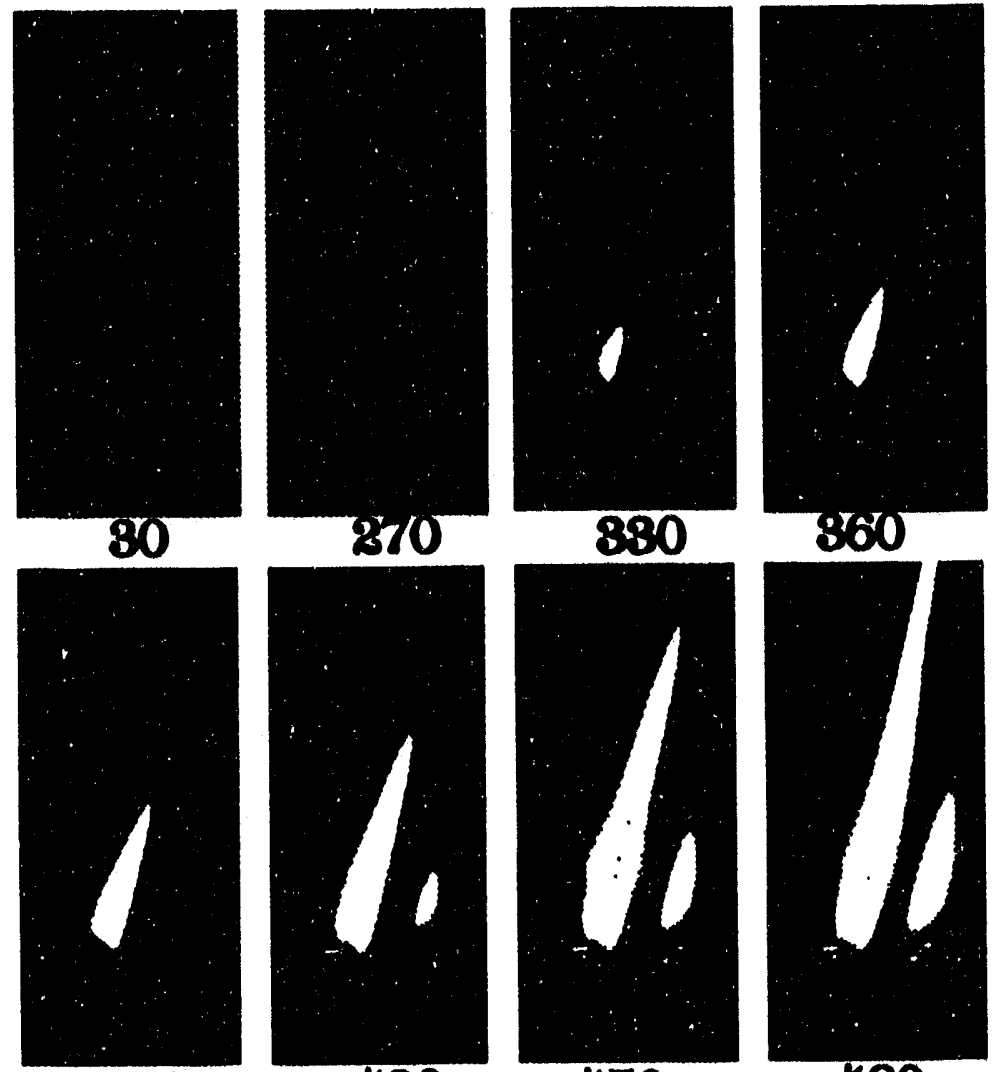

860
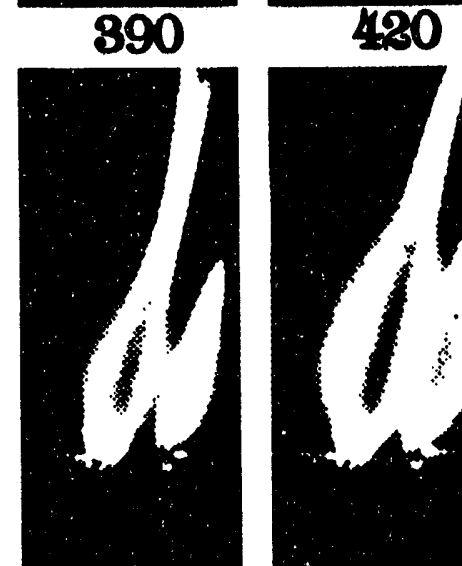

510

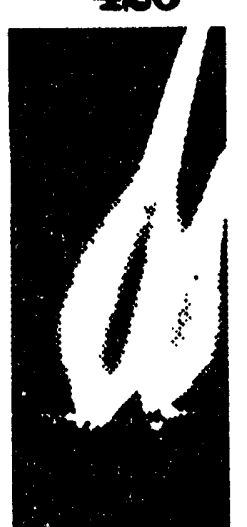

540

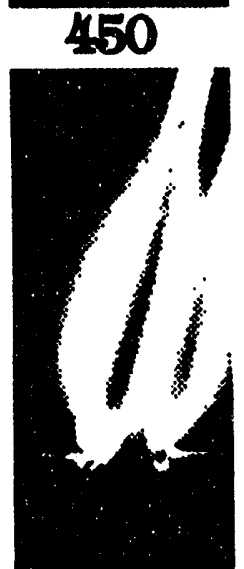

570

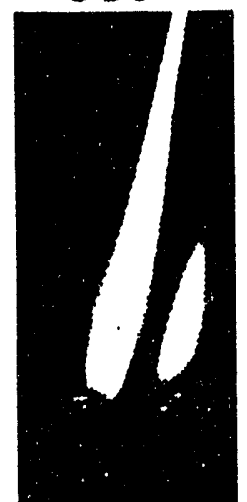

480

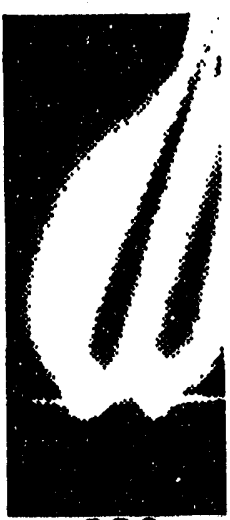

630

Figure 44a. Ignition and Combustion Sequence for Large High Volatile Coal Two-Particle Array $(l=4.86 \mathrm{~mm})$ 

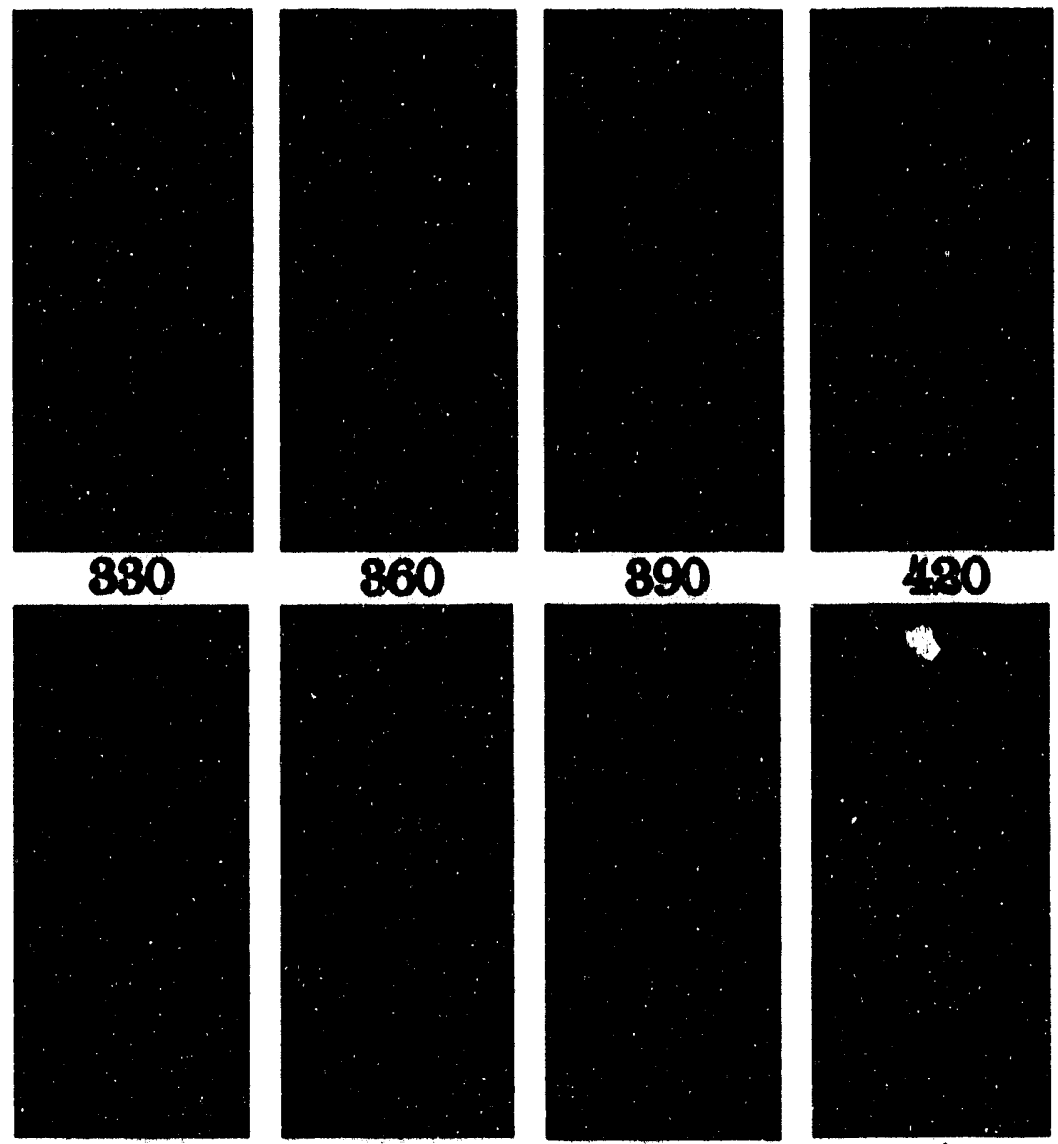

450

480

510
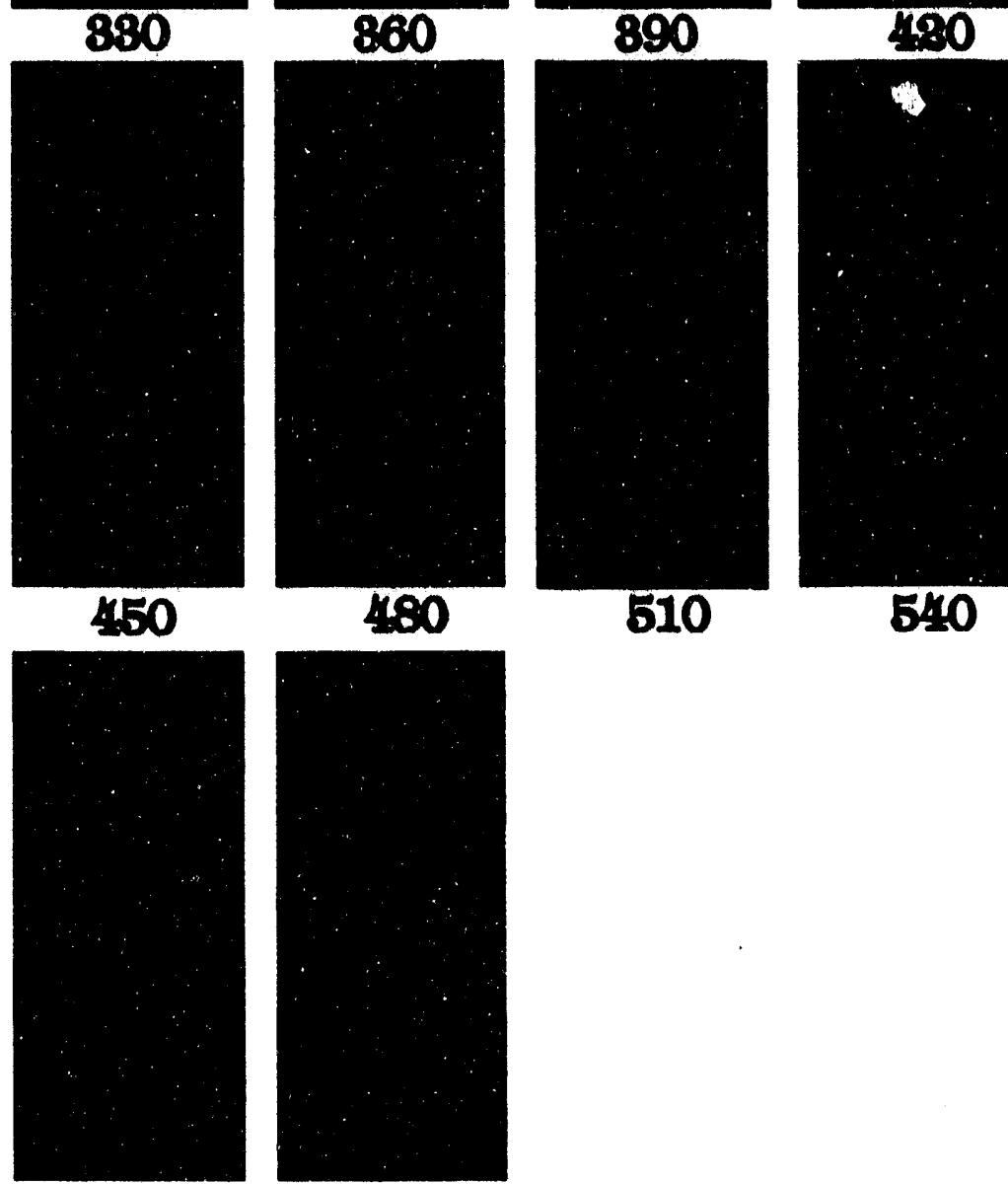

540

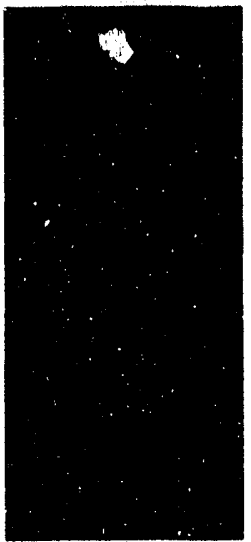

570

680

Figure 44b. Images of Ignition and Combustion Sequence With Boundary Detection Function $(l=4.86 \mathrm{~mm})$ 

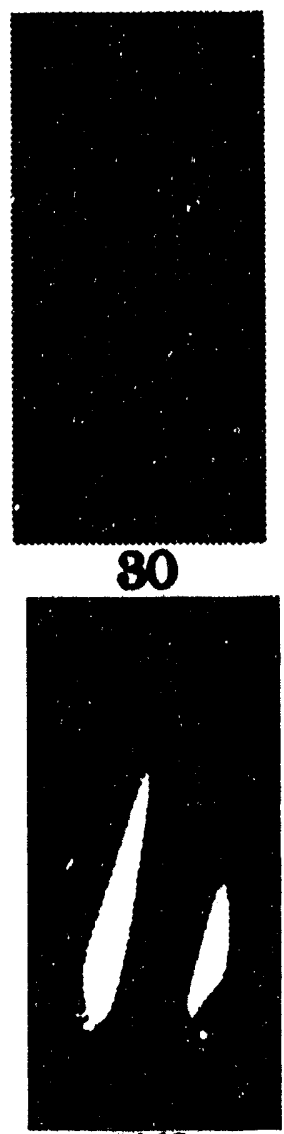

870

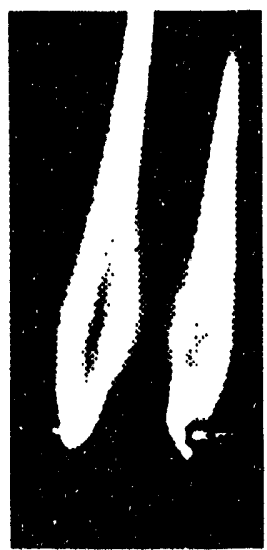

490

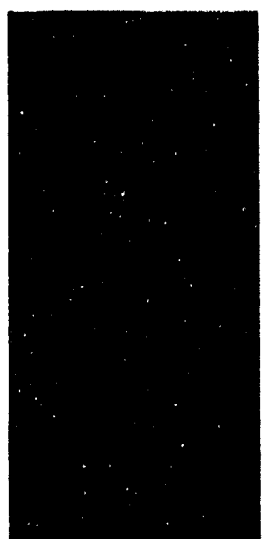

150

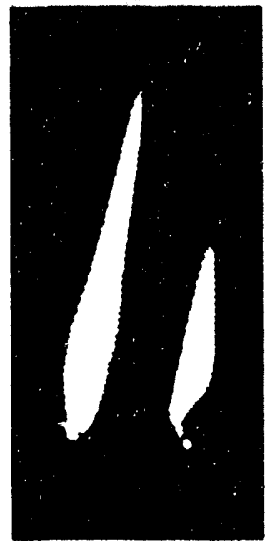

800

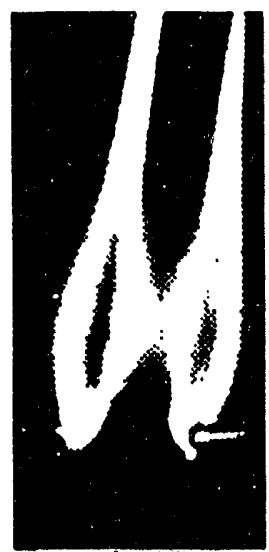

450

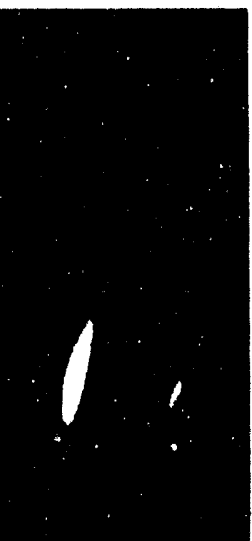

810

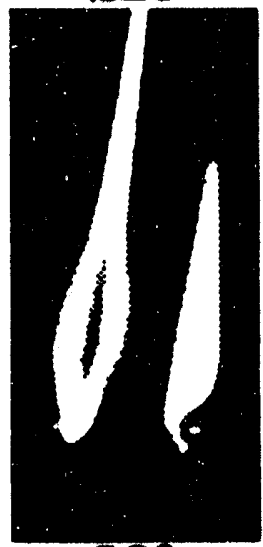

860

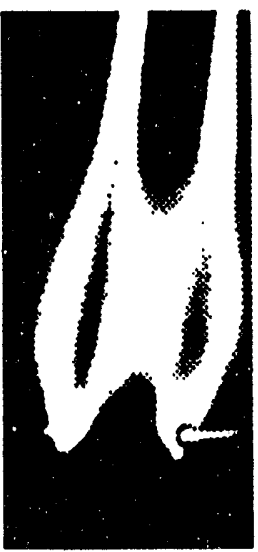

480

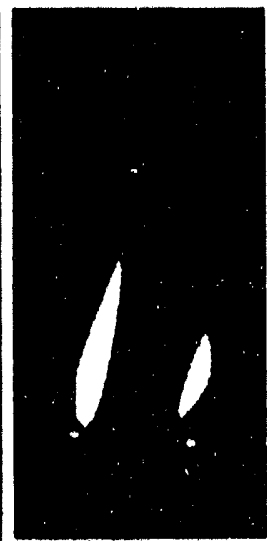

$2 M 0$

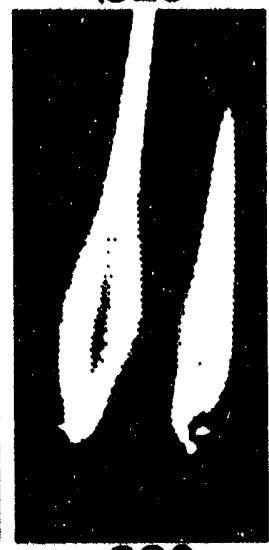

890

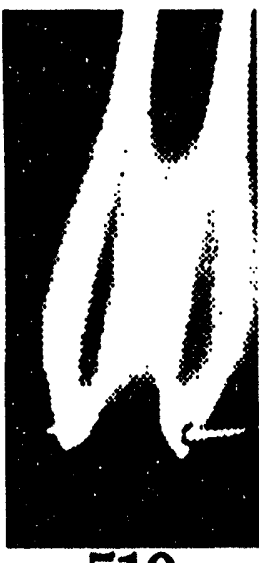

510

Figure 45a. Ignition and Combustion Sequence for Large High Volatile

Coal Two-Particle Array $(l=7.26 \mathrm{~mm})$ 

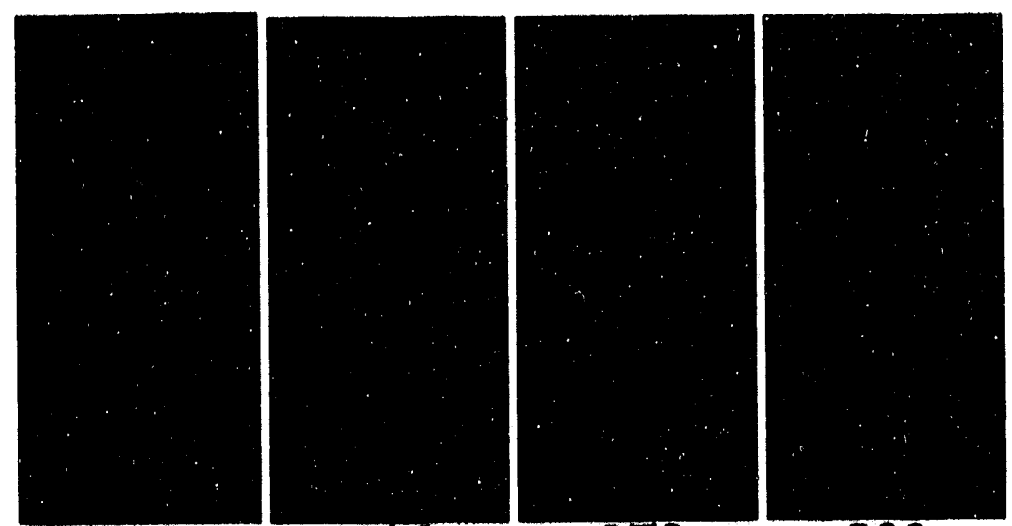

810

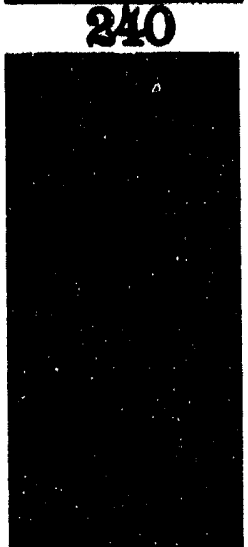

870

800
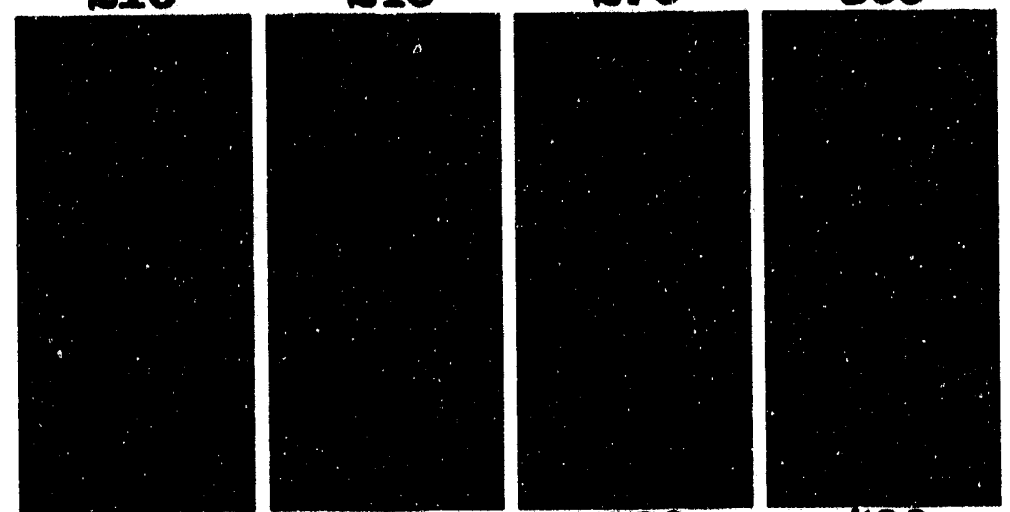

890

860

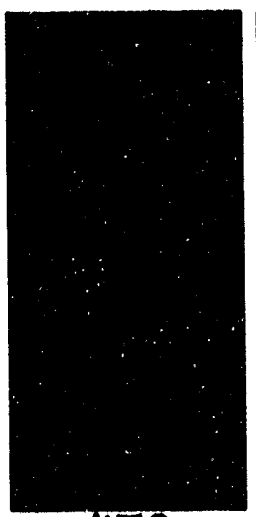

450

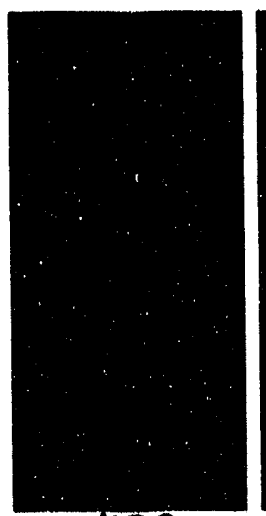

480

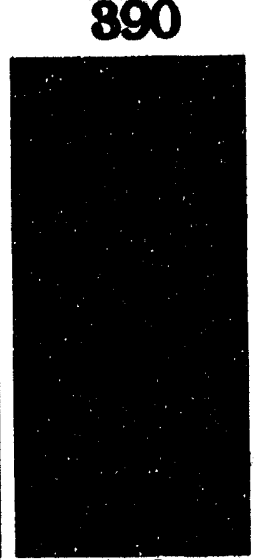

510

540

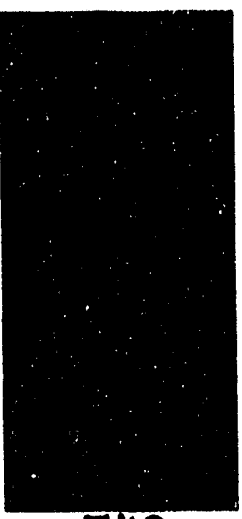

Figure 45b. Images of Ignition and Combustion Sequence With Boundary Detection Function $(l=7.26 \mathrm{~mm})$ 

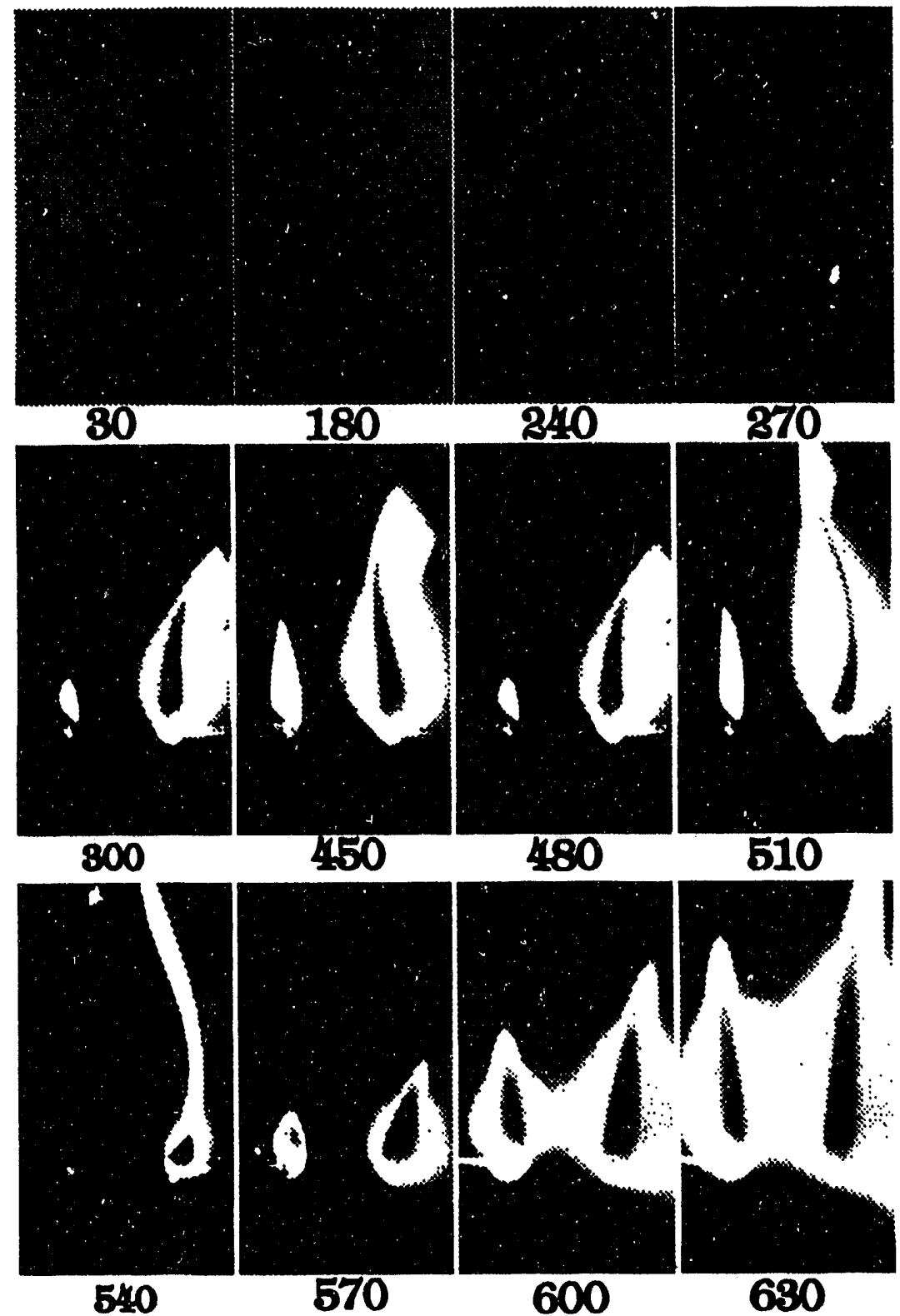

Figure 46a. Ignition and Combustion Sequence for Large High Volatile

Coal Two-Particle Array $(l=10.60 \mathrm{~mm})$ 

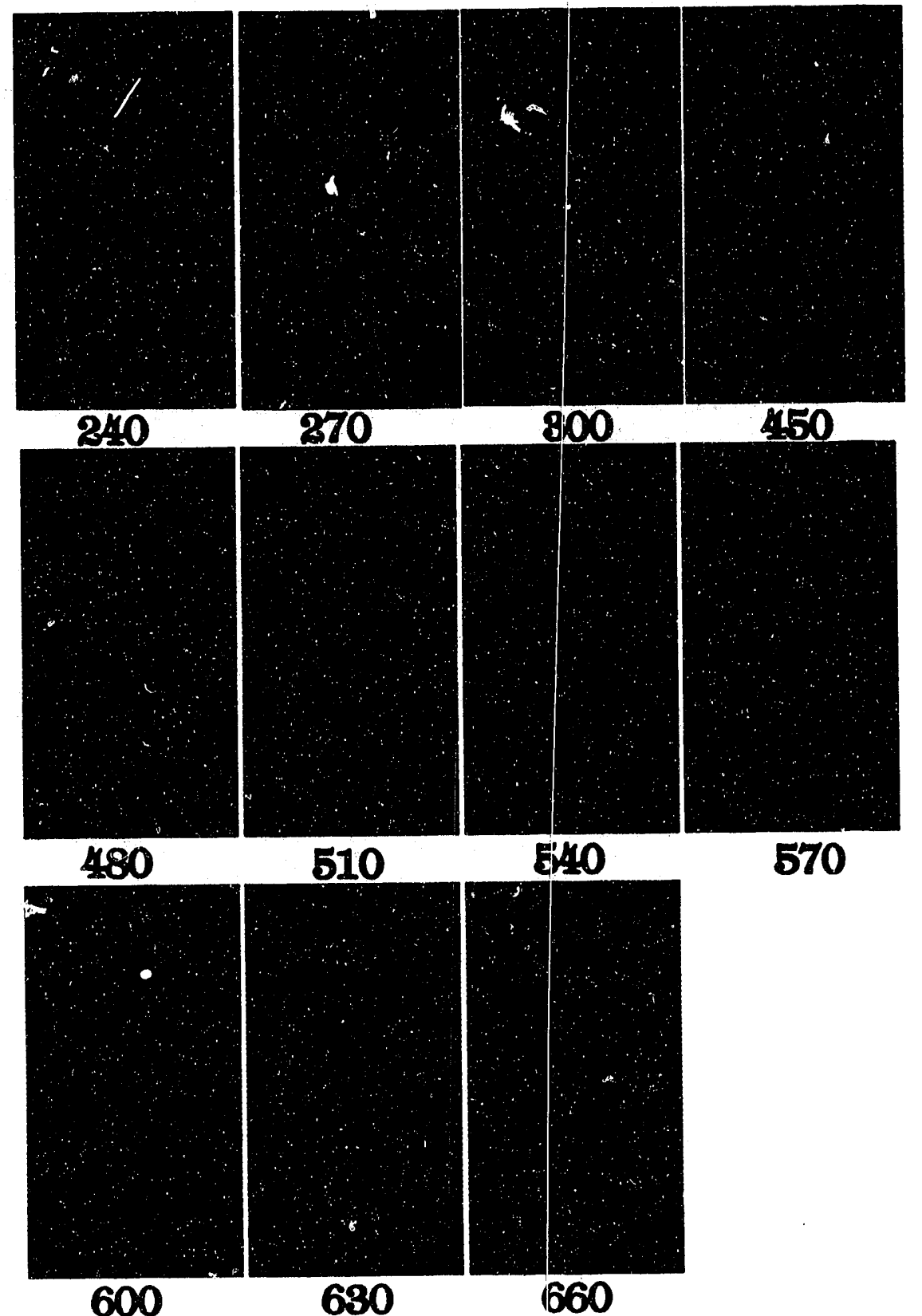

570

Figure 46b. Images of Ignition and Combustion Sequence With Boundary Detection Function $(l=10.60 \mathrm{~mm})$ 


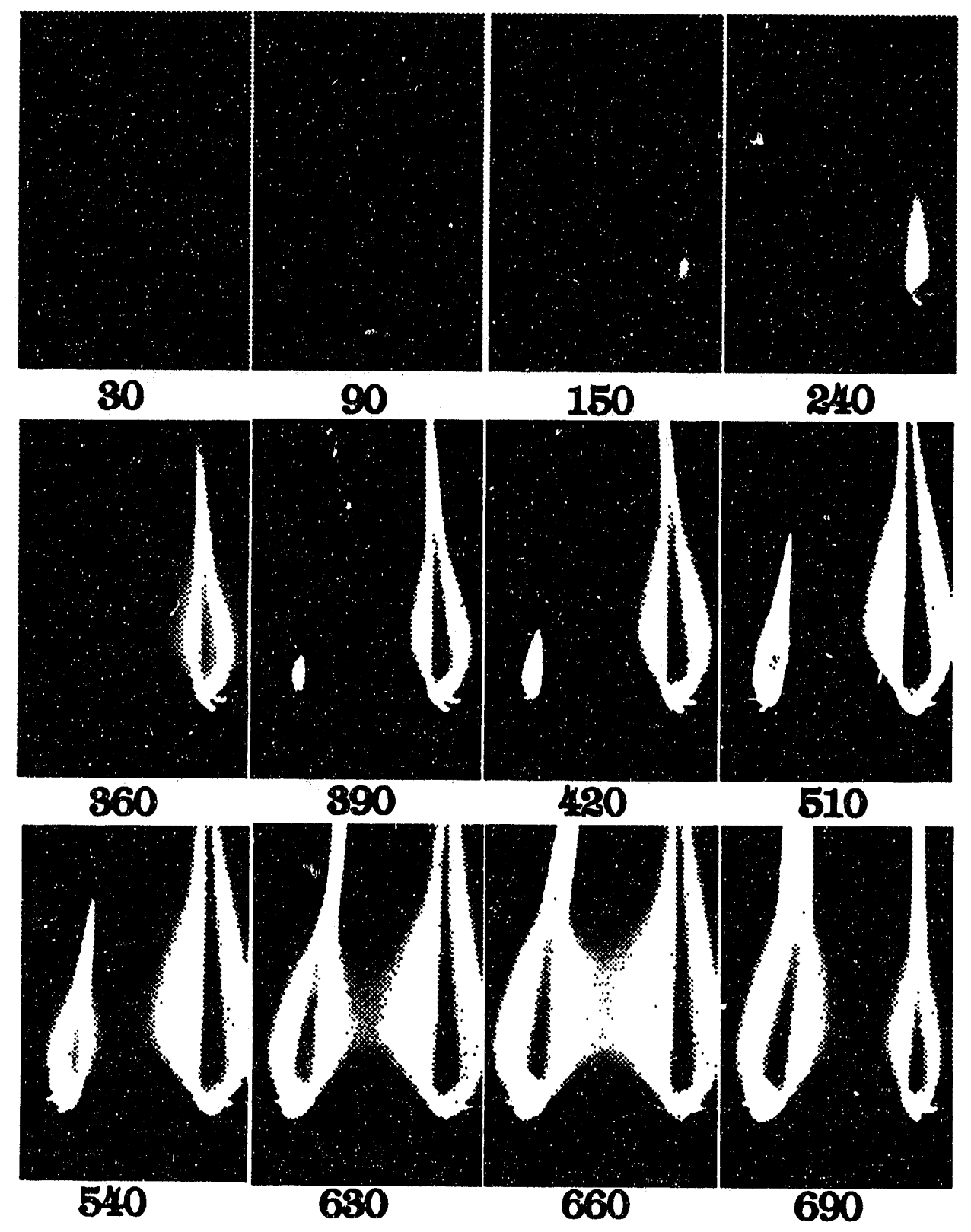

Figure 47a. Ignition and Combustion Sequence for Large High Volatile Coal Two-Particie Array $(l=13.45 \mathrm{~mm})$ 


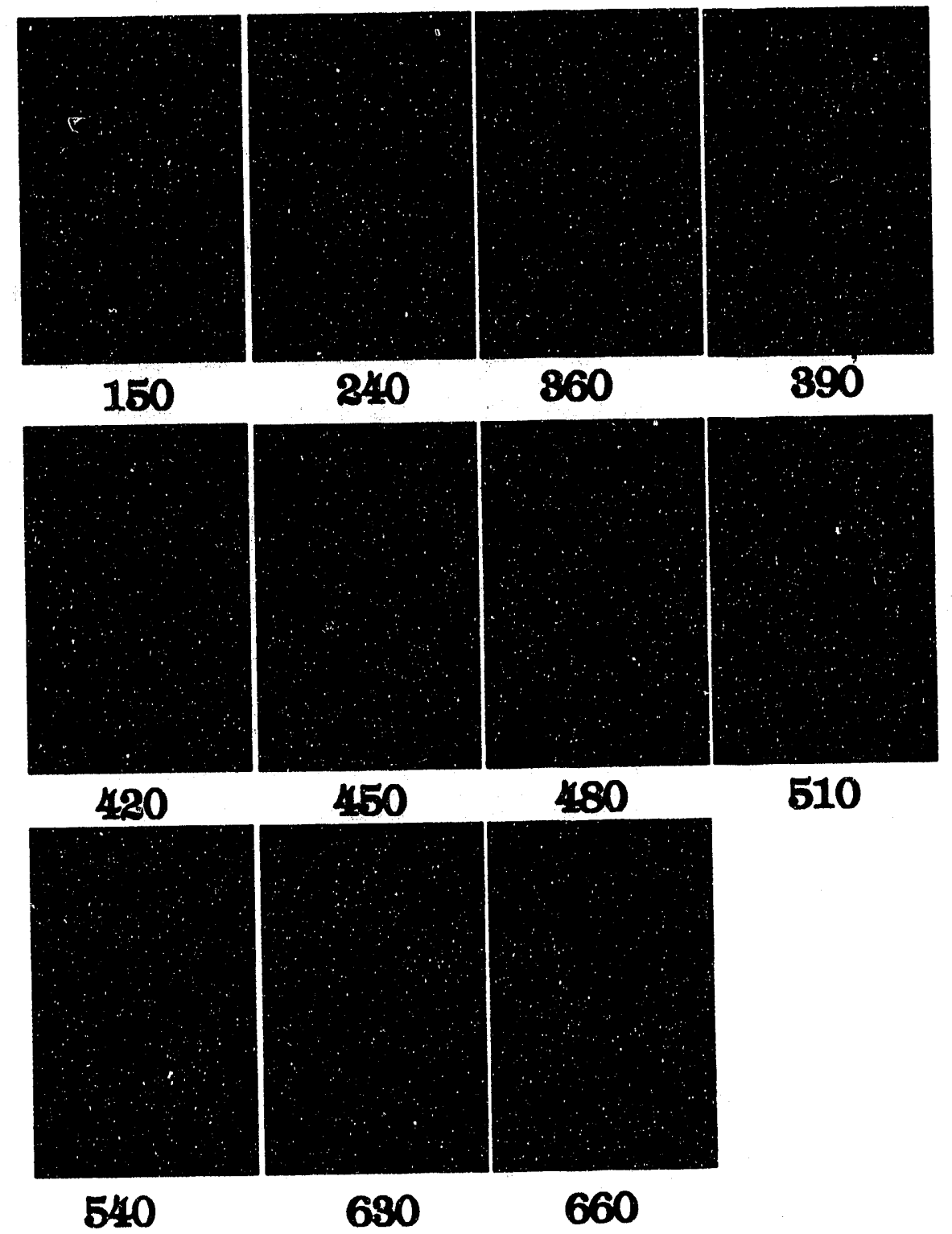

Figure 47b. Images of Ignition and Combustion Sequence With Boundary Detection Function $(l=13.45 \mathrm{~mm})$ 

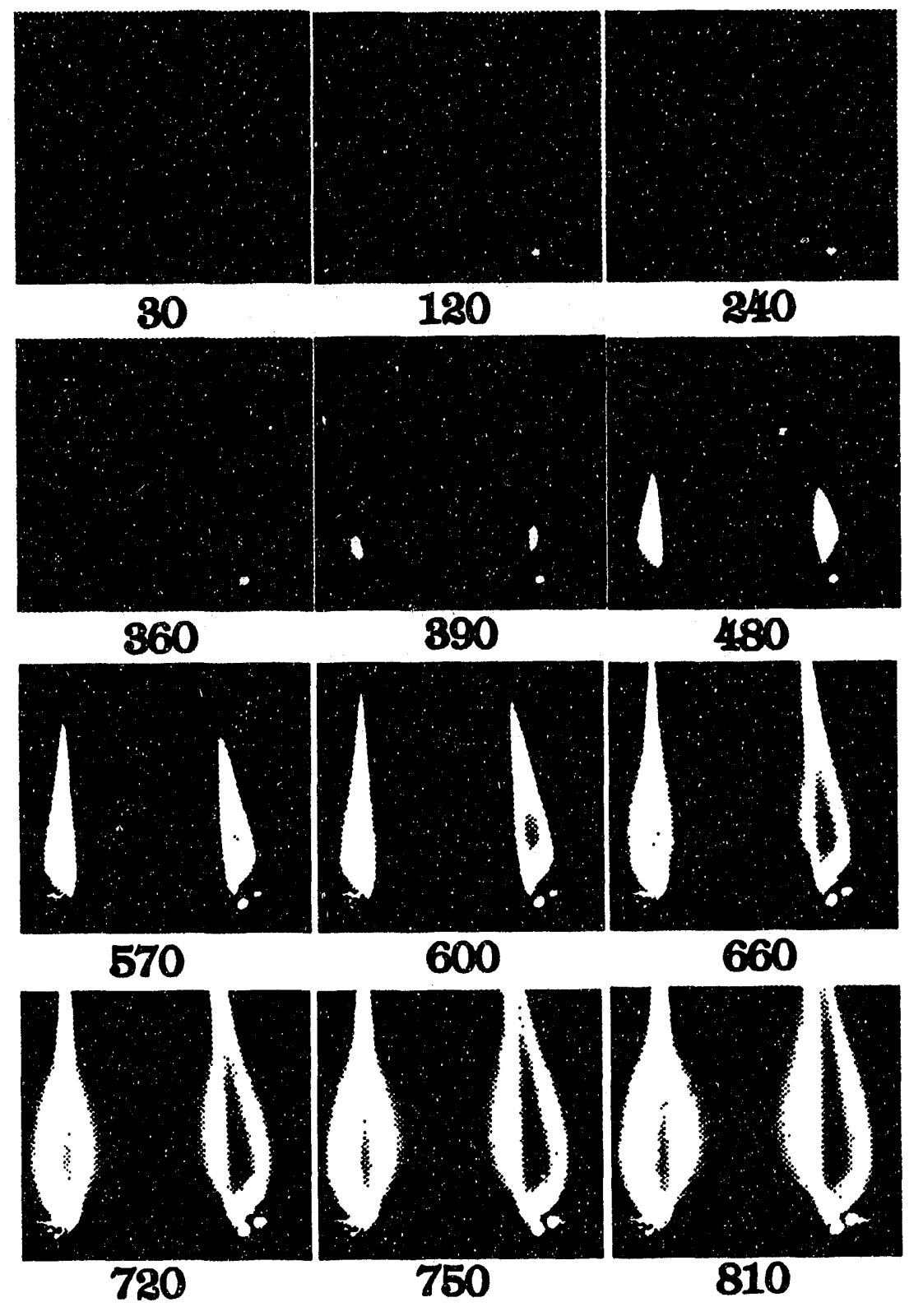

Figure 48a. Ignition and Combustion Sequence for Large High Volatile

Coal Two-Particle Array $(l=15.88 \mathrm{~mm})$ 


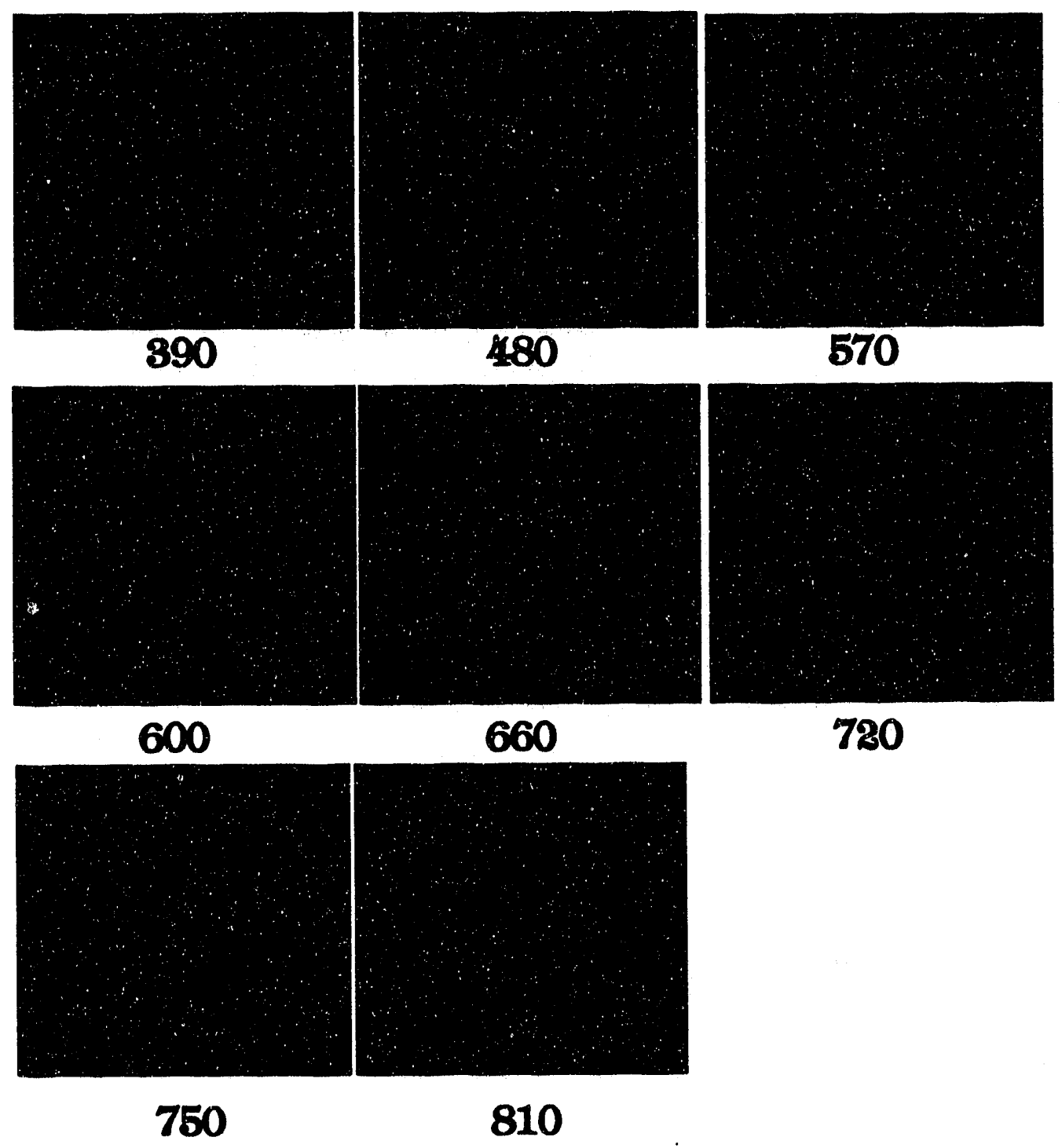

Figure 48b. Images of Ignition and Combustion Sequence With Boundary Detection Function $(l=15.88 \mathrm{~mm})$ 


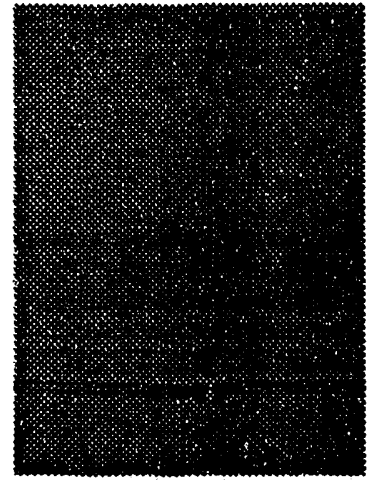

30

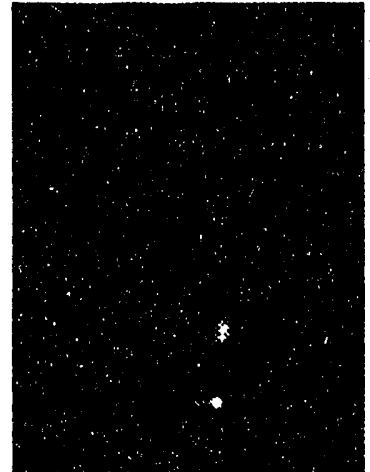

$\mathbf{2 7 0}$

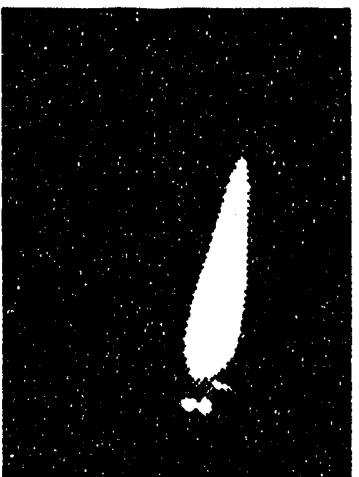

450

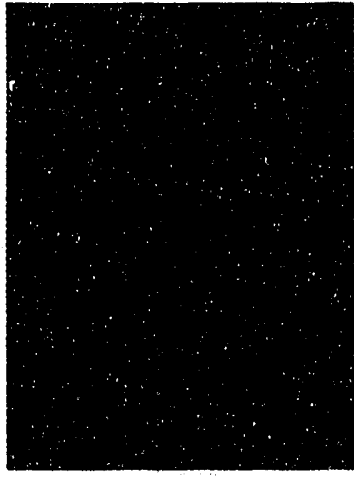

180

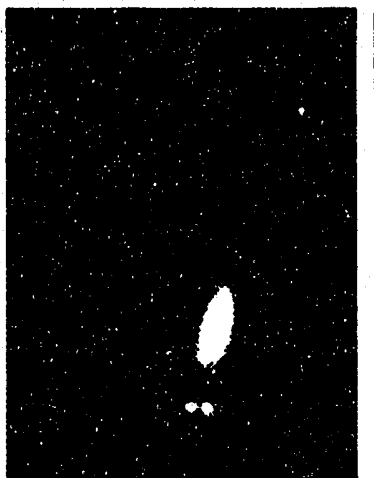

330

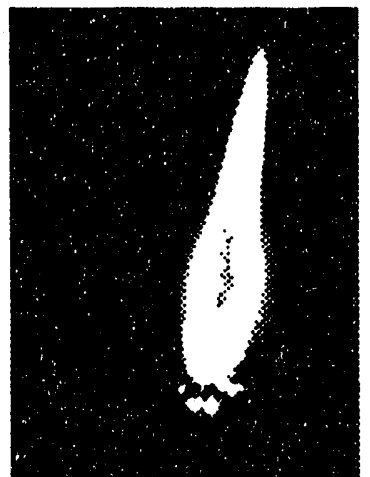

510

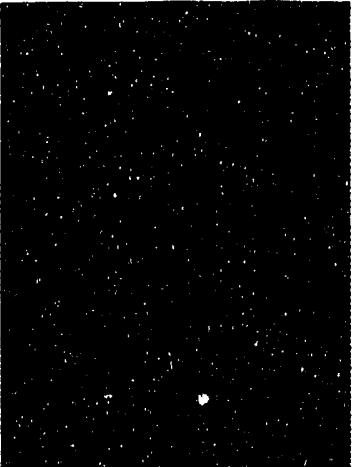

210

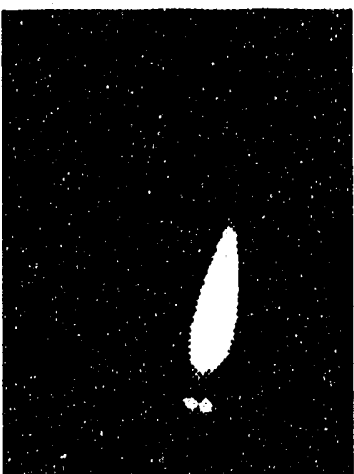

390

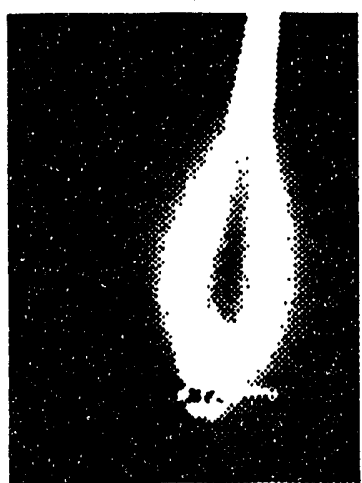

570

Figure 49a. Ignition and Combustion Sequence for Intermediate High Volatile

Coal Two-Particle Array $(l=2.13 \mathrm{~mm})$ 


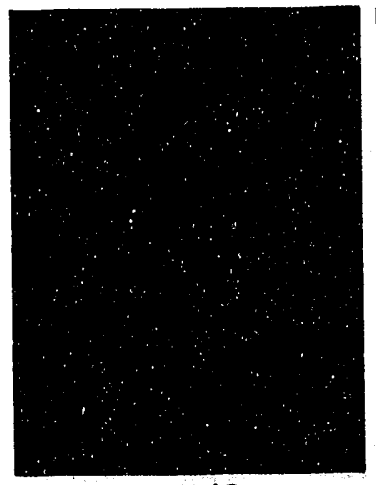

270

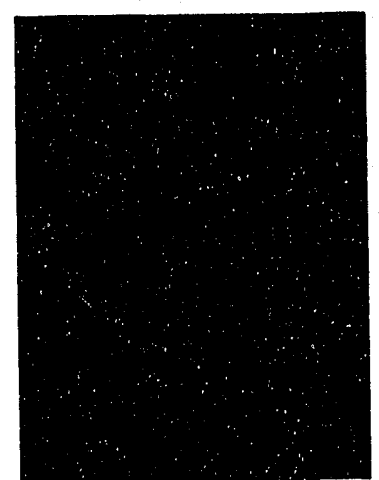

450

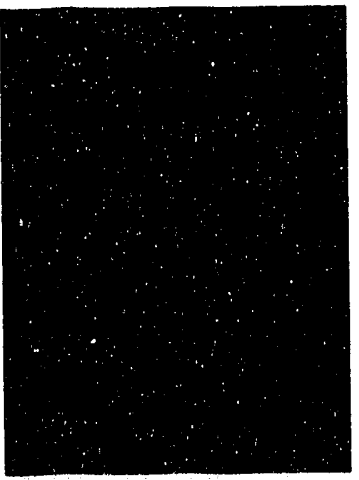

330

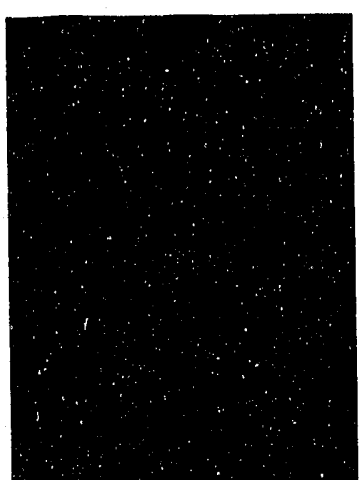

510

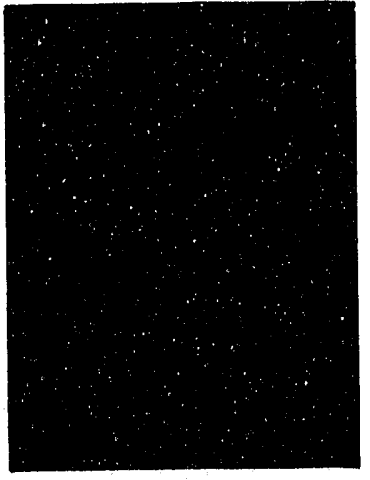

390

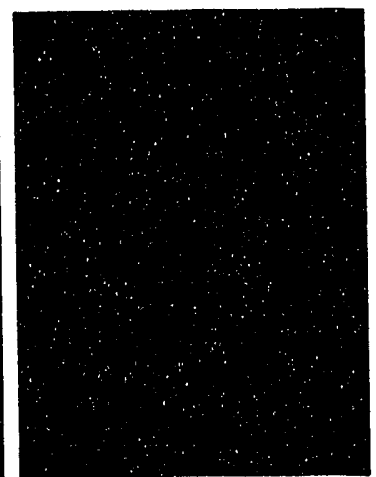

570

Figure 49b. Images of Ignition and Combustion Sequence With Boundary Detection Function $(l=2.13 \mathrm{~mm})$ 

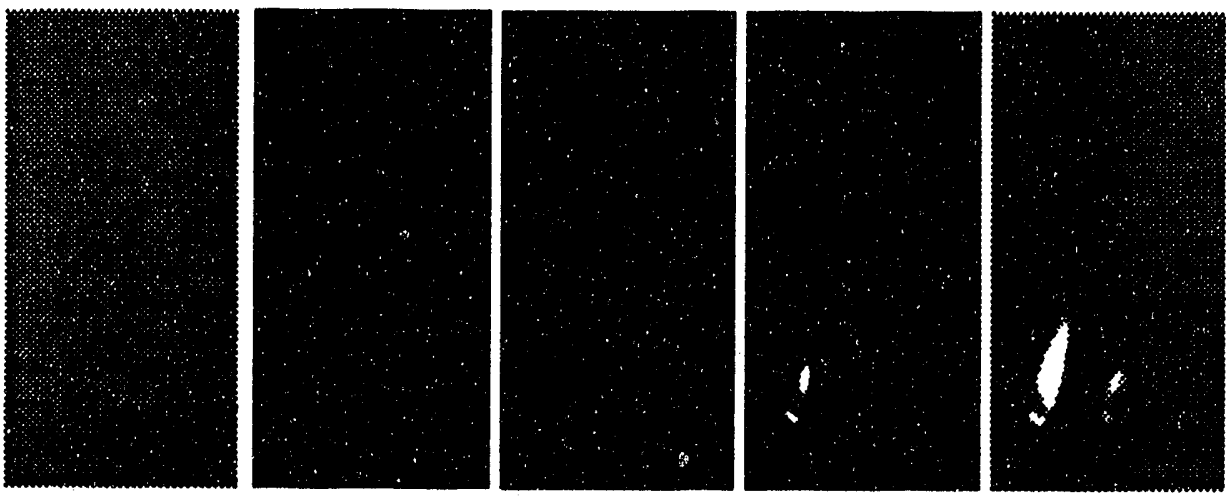

30

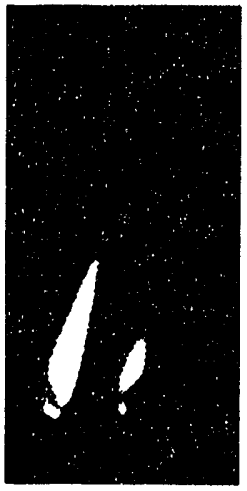

120

180
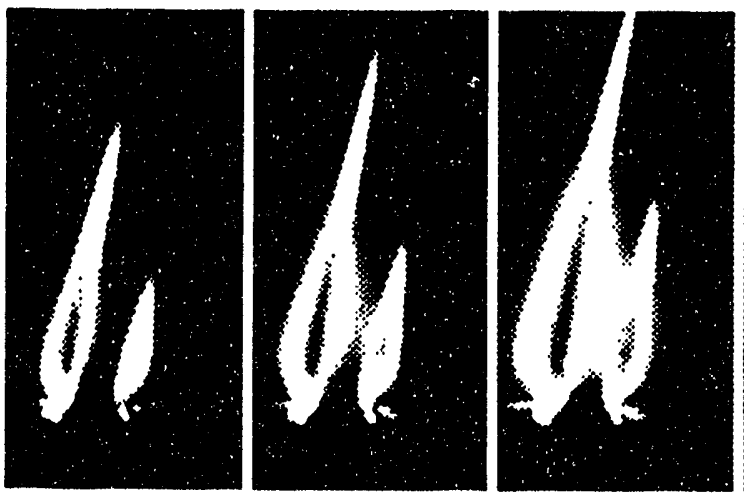

480 $\mathbf{8 7 0}$

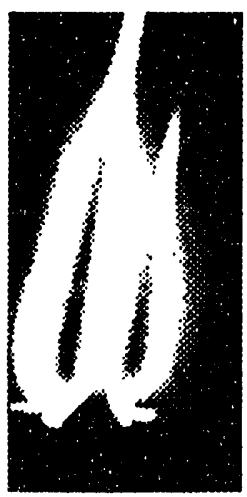

480

Figure 50a. Ignition and Combustion Sequence for Intermediate High Volatile Coal Two-Particle Array $(l=4.53 \mathrm{~mm})$ 

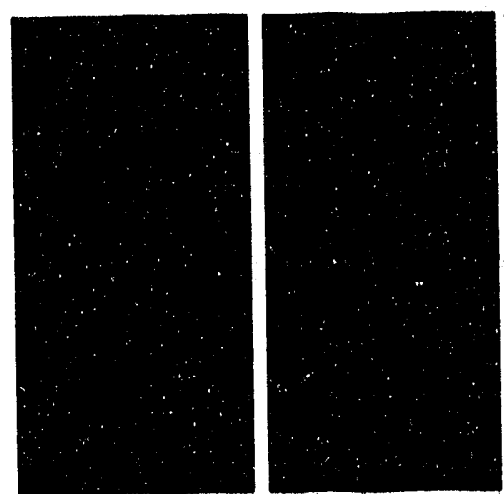

240

270

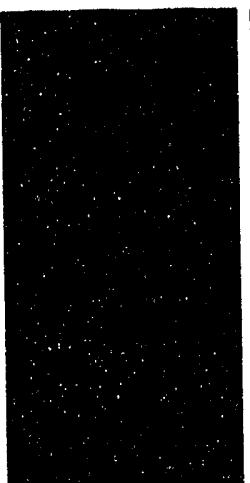

890

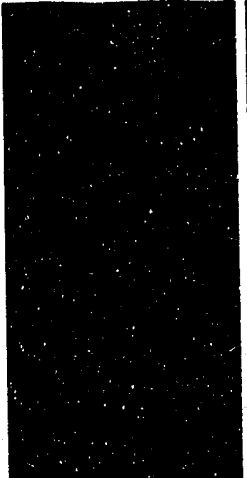

420

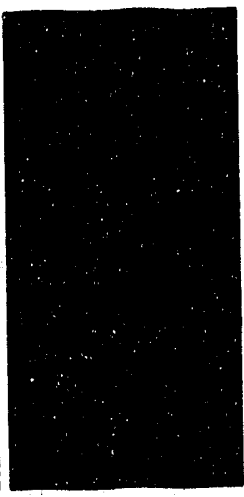

800

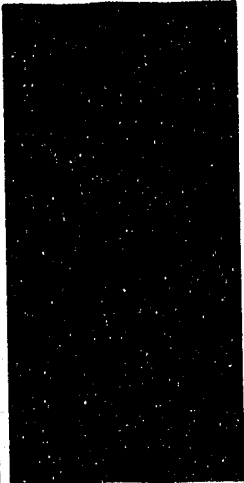

480

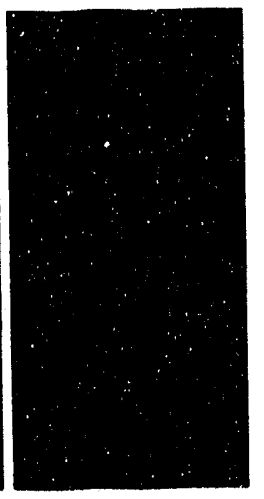

860

Figure 50b. Images of Ignition and Combustion Sequence With Boundary Detection Function $(l=4.53 \mathrm{~mm})$ 

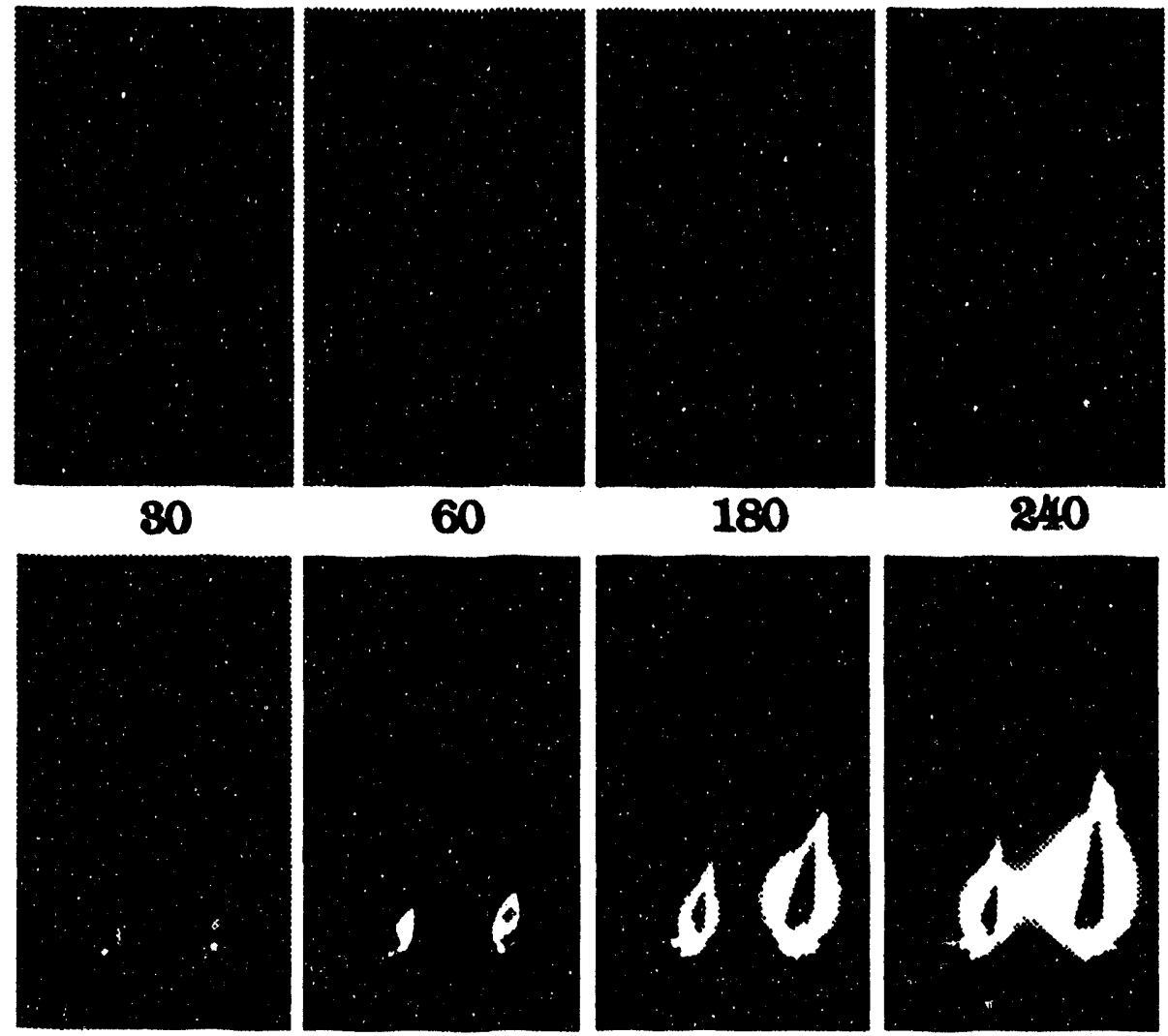

\section{0}

180

240
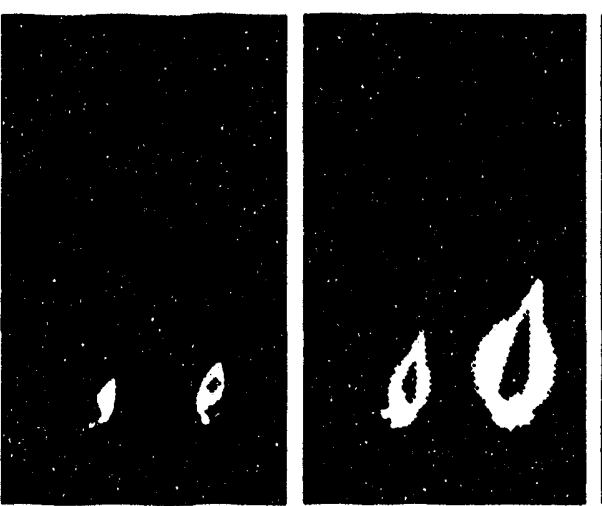

860

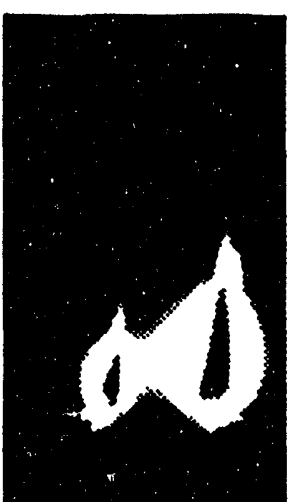

$\mathbf{8 0 0}$

890

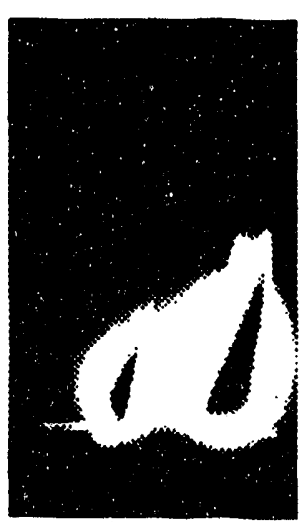

540

Figure 51a. Ignition and Combustion Sequence for Intermediate High Volatile Coal Two-Particle Array $(l=7.12 \mathrm{~mm})$ 


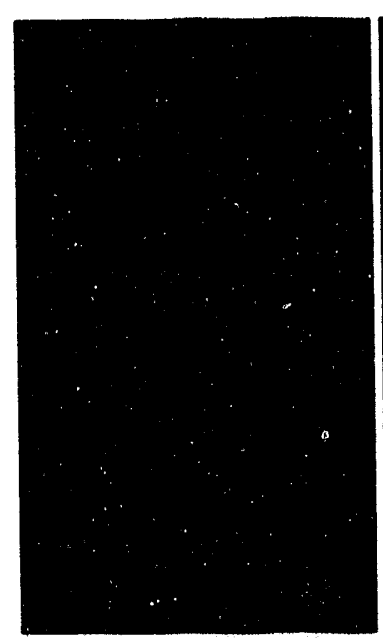

240

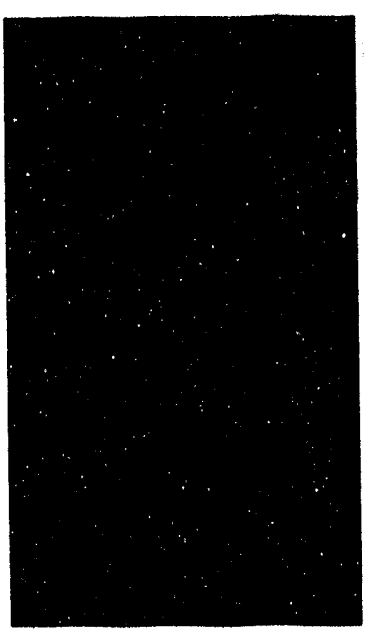

860

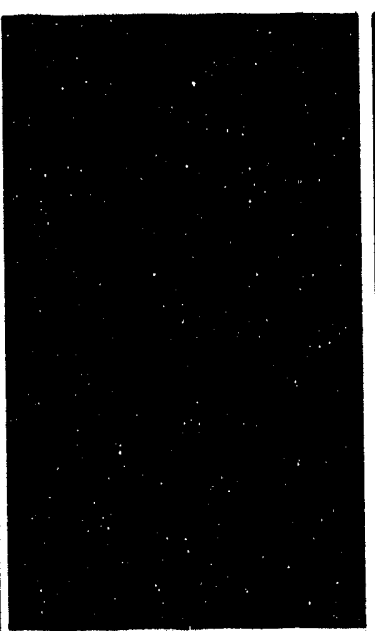

270

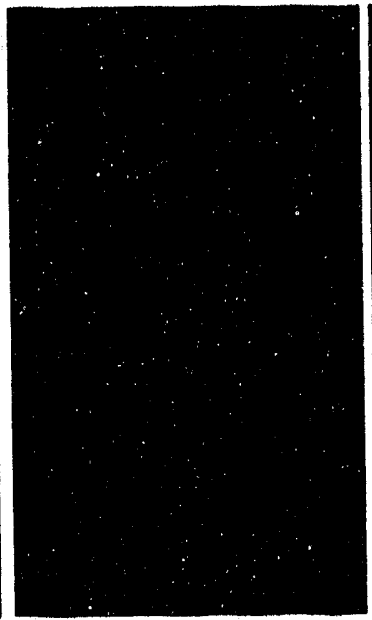

890

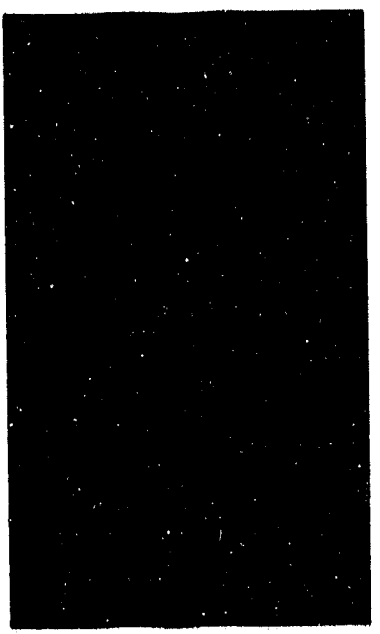

800

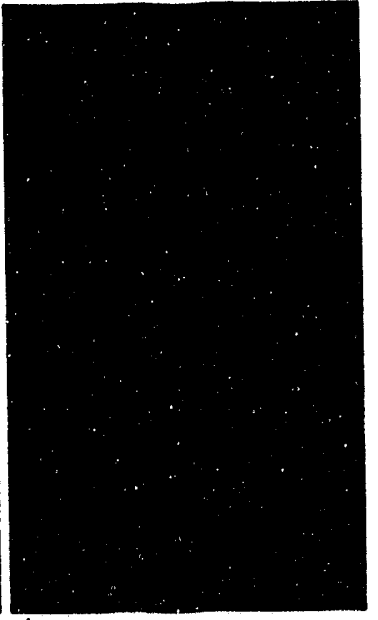

540

Figure 51b. Images of Ignition and Combustion Sequence With Boundary Detection Function $(l=7.12 \mathrm{~mm})$ 


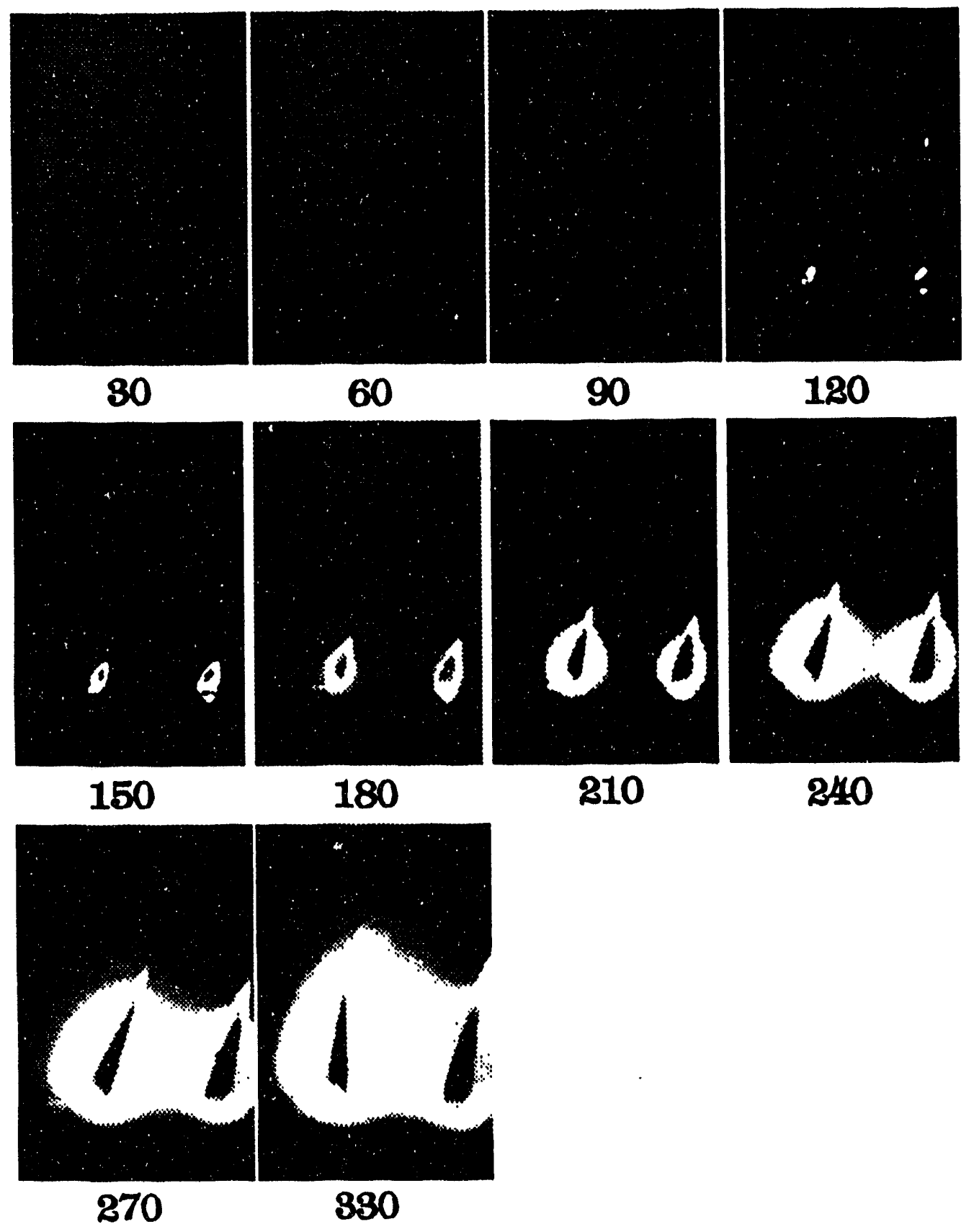

Figure 52a. Ignition and Combustion Sequence for Intermediate High Volatile Coal Two-Particle Array $(l=10.34 \mathrm{~mm})$ 


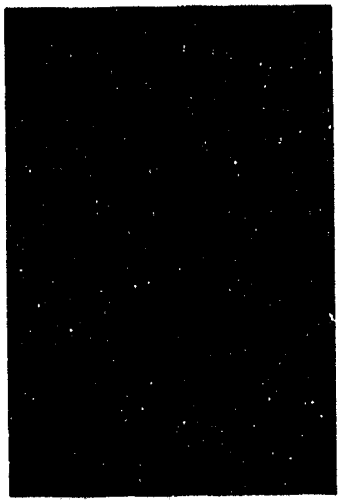

120

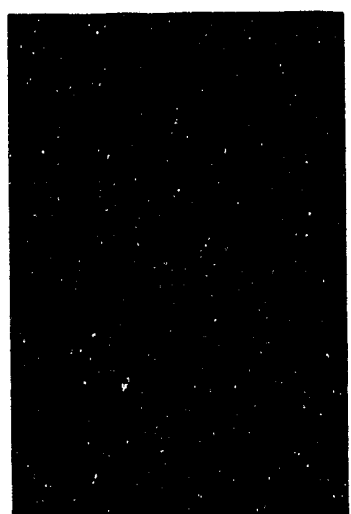

210

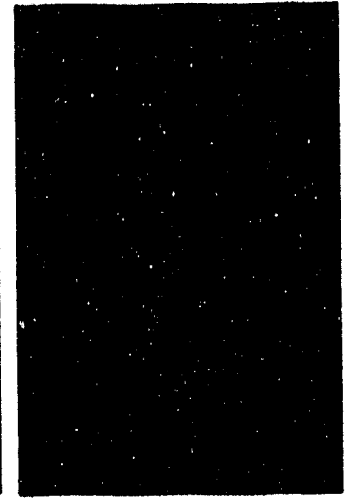

150

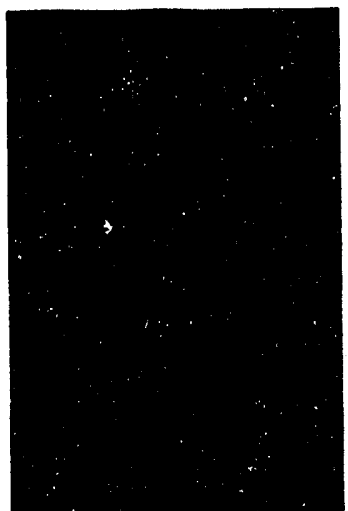

270

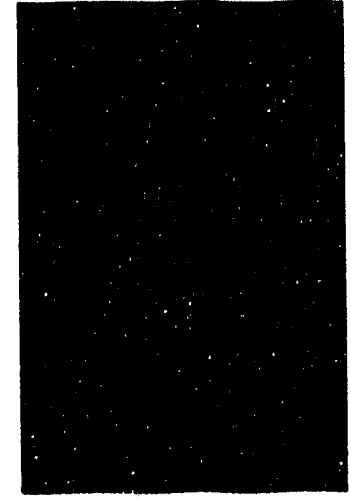

180

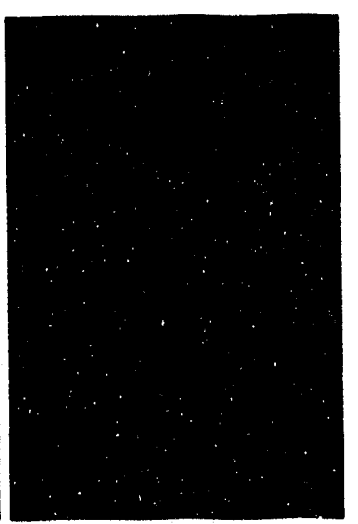

300

Figure 52b. Images of Ignition and Combustion Sequence With Boundary Detection Function $(l=10.34 \mathrm{~mm})$ 


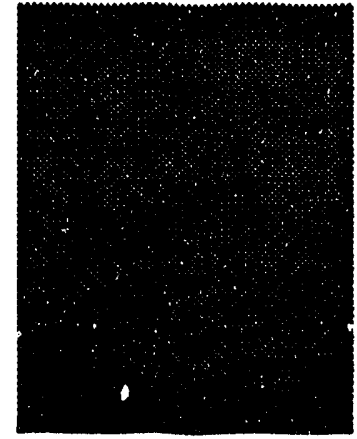

30

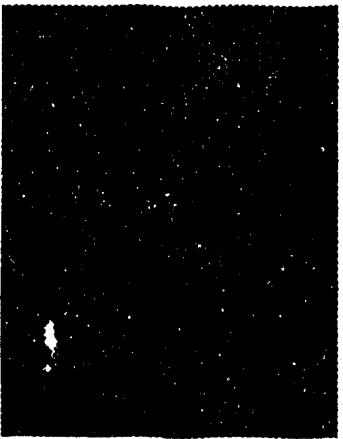

450

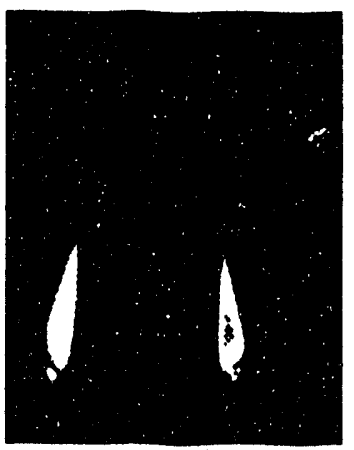

570

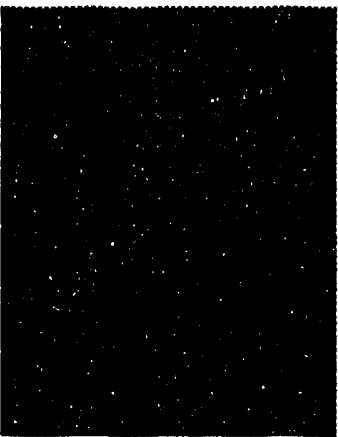

270

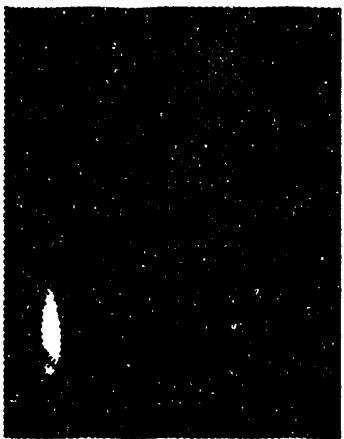

480

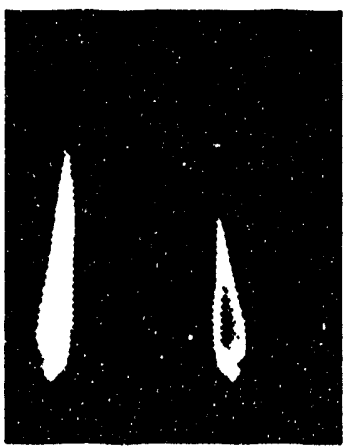

600

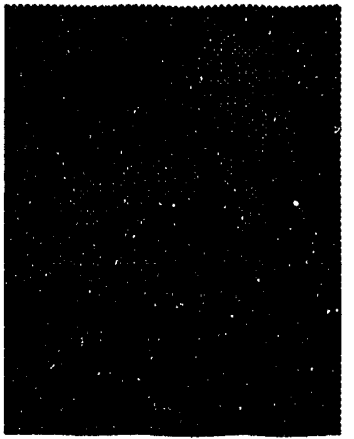

390

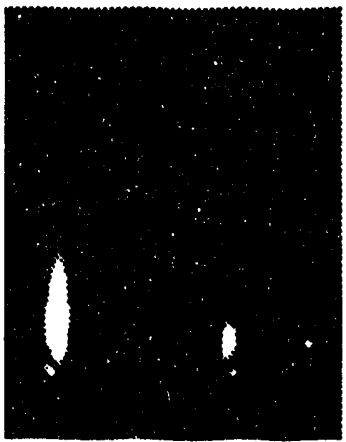

510

Figure 53a. Ignition and Combustion Sequence for Intermediate High Volatile

Coal Two-Particle Array $(l=13.42 \mathrm{~mm})$ 


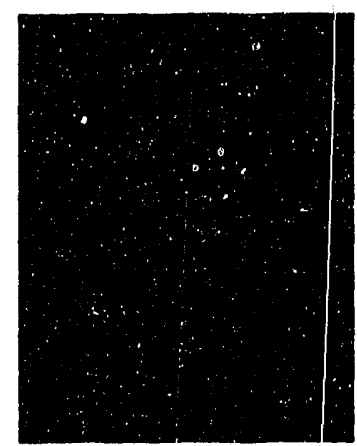

480

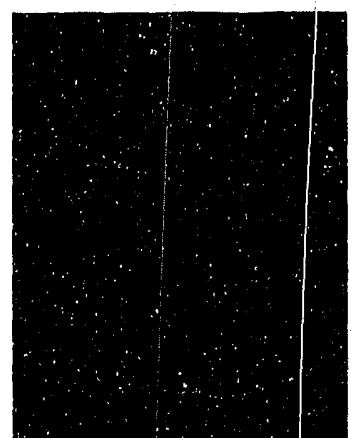

570

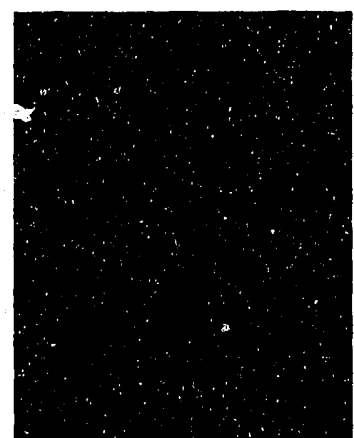

510

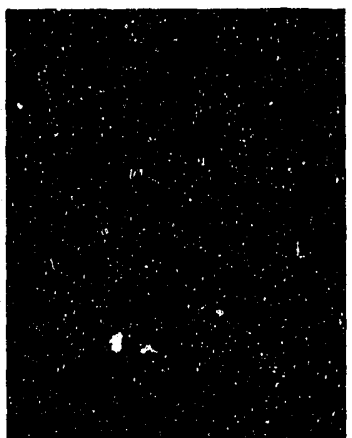

600

Figure 53b. Images of Ignition and Combustion Sequence with Boundary Detection Function $(l=13.42 \mathrm{~mm})$ 

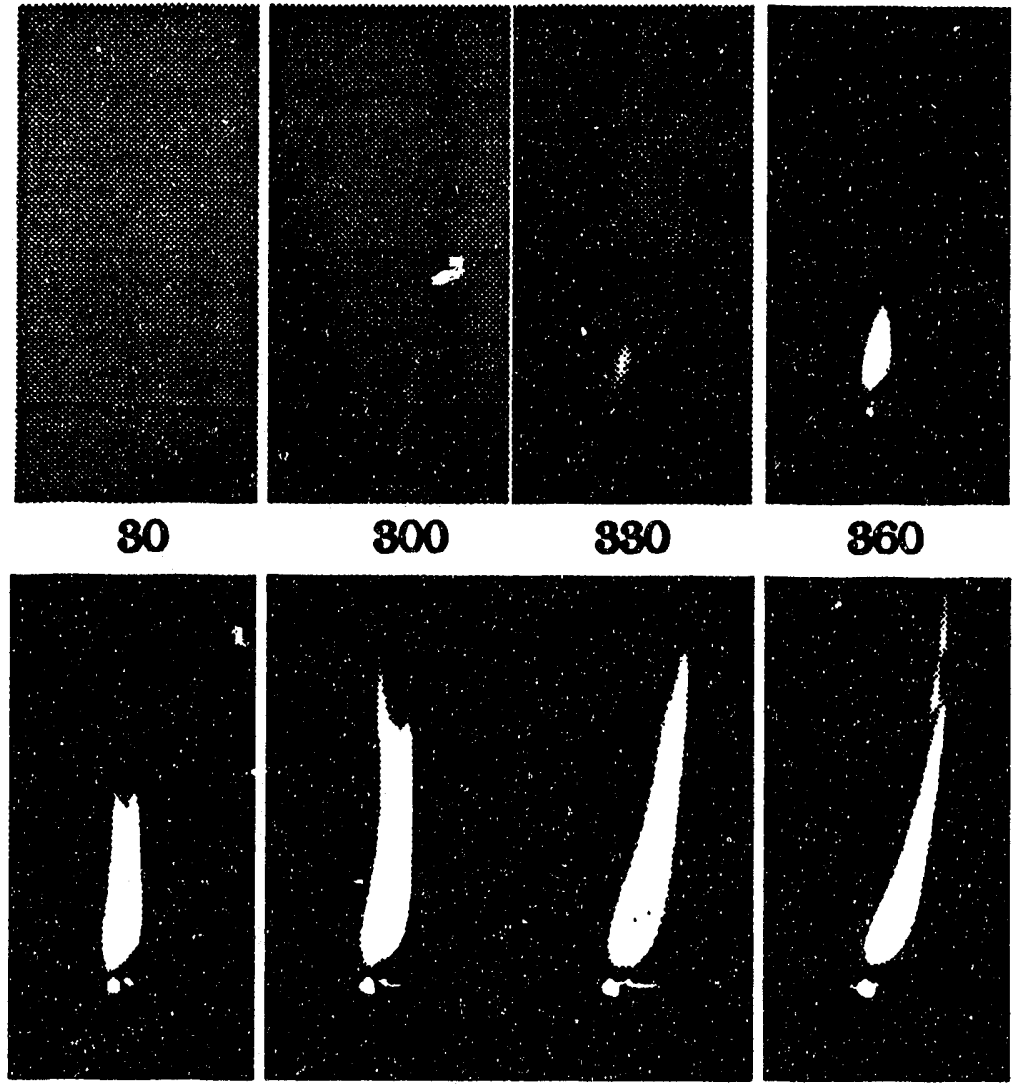

$$
800
$$

$\mathbf{8 8 0}$

860
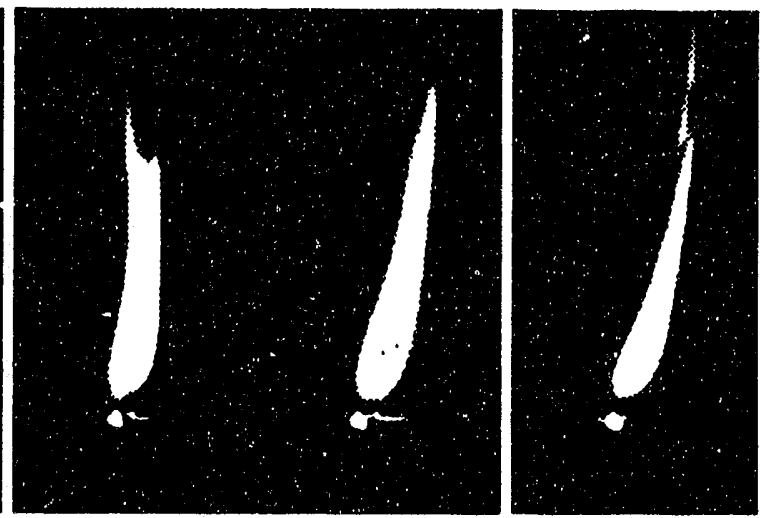

480

480

540

570
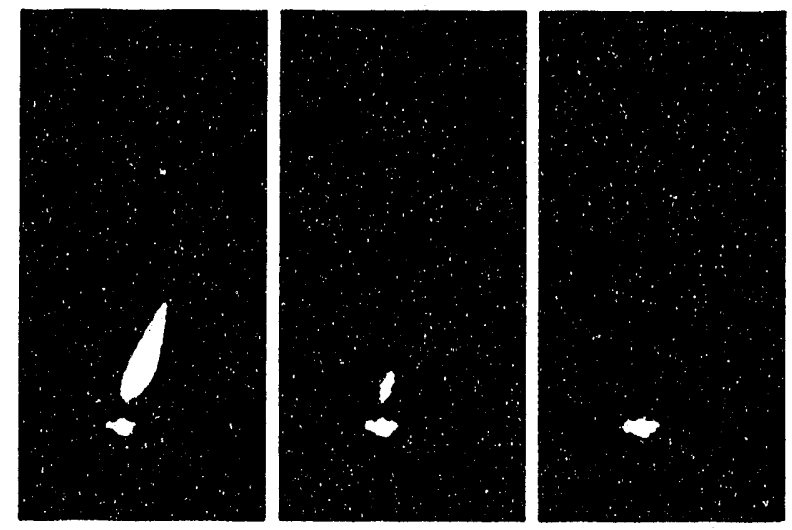

600

630

660

Figure 54a. Ignition and Combustion Sequence for Small High Volatile Coal Two-Particle Array $(l=2.05 \mathrm{~mm})$ 


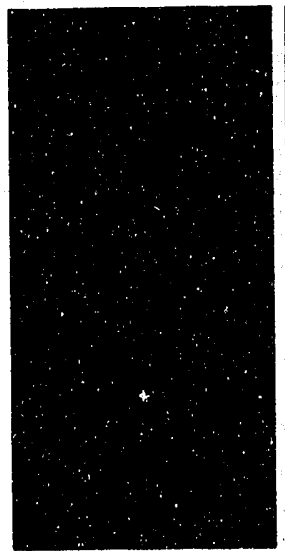

360

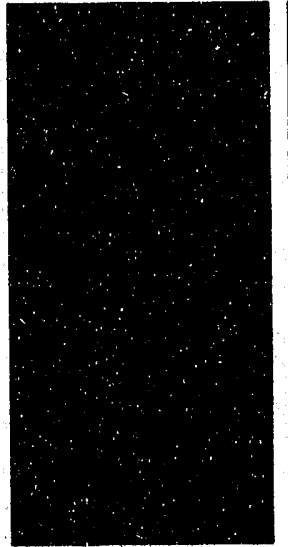

480

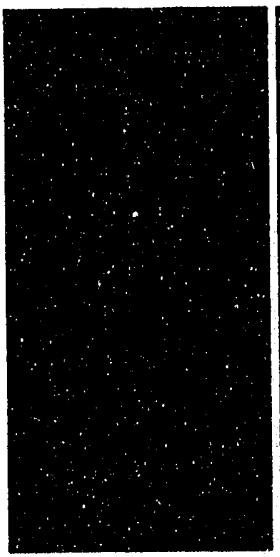

570

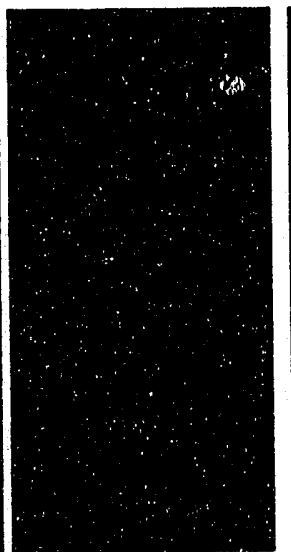

600

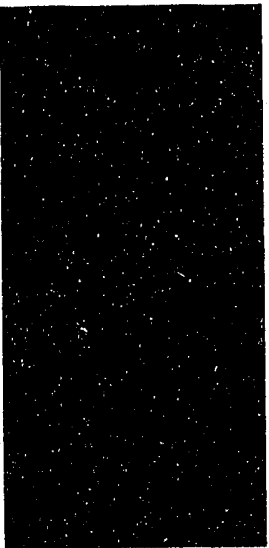

480

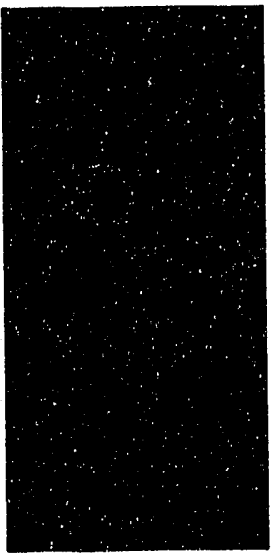

630

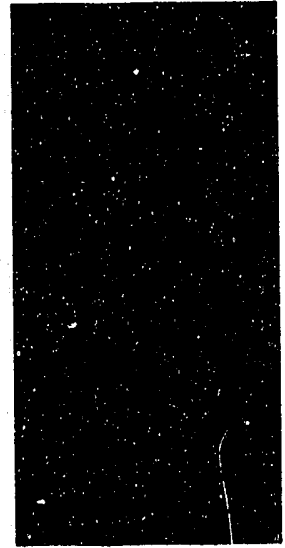

540

Figure 54b. Images of Ignition and Combustion Sequence with Boundary Detection Function $(l=2.05 \mathrm{~mm})$ 

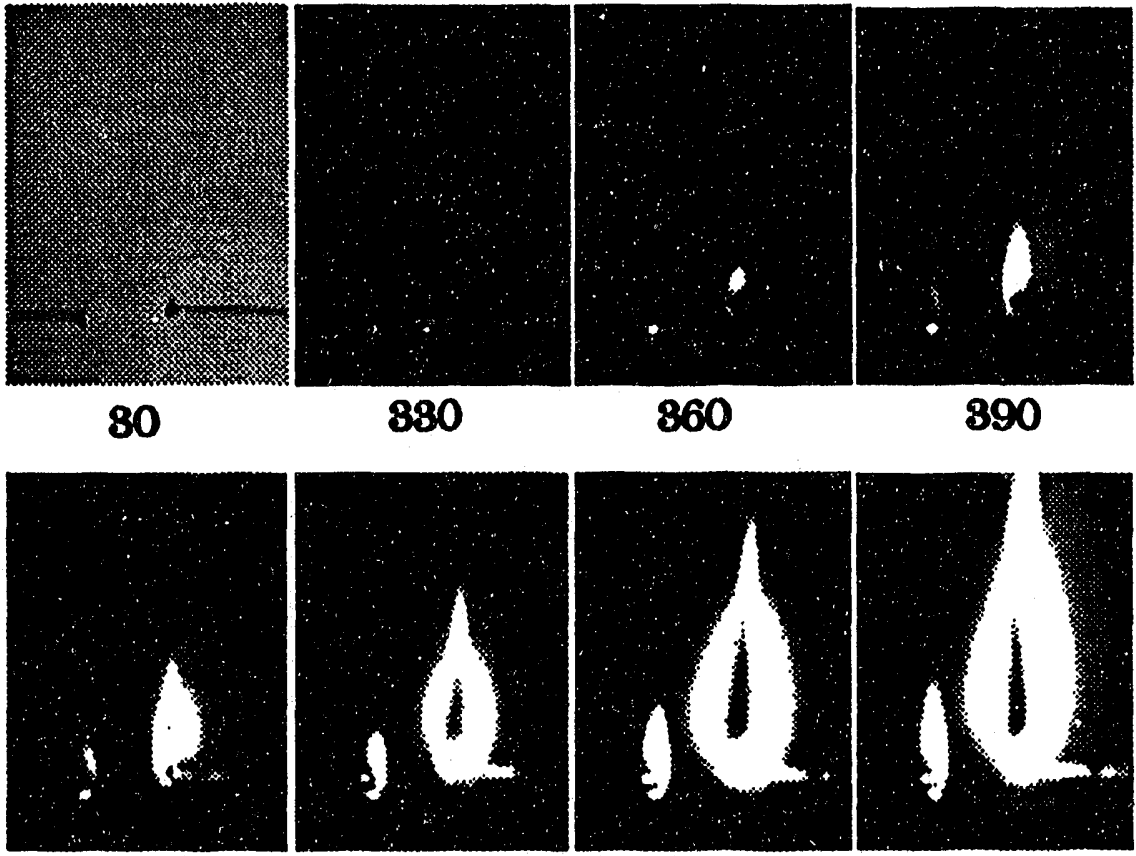

480

450

510

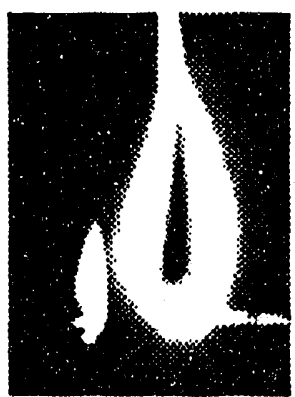

540

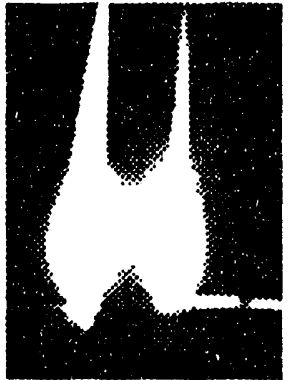

720

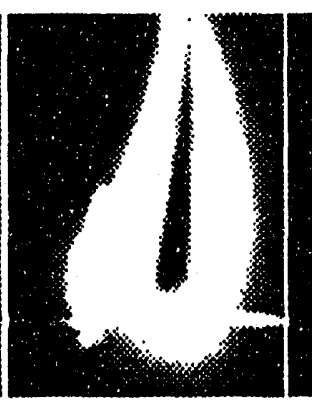

570

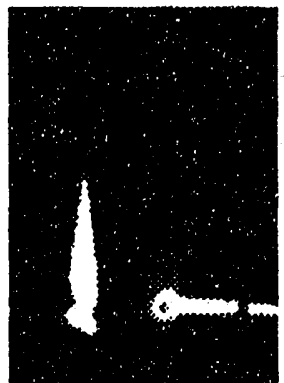

870

480

.

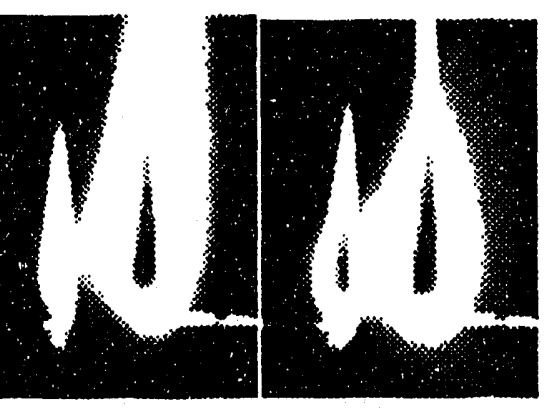

690

660

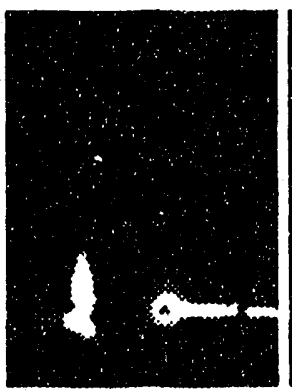

$\mathbf{9 0 0}$

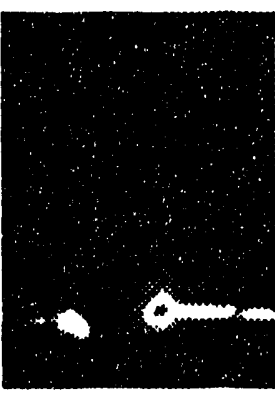

930

Figure 55a. Ignition and Combustion Sequence for Small High Volatile

Coal Two-Particle Array $(l=4.67 \mathrm{~mm})$ 

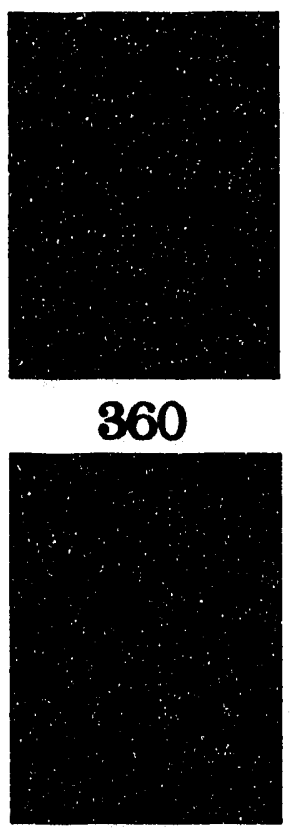

480

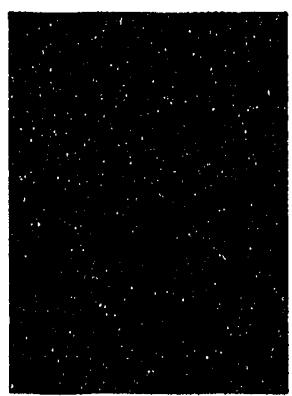

630

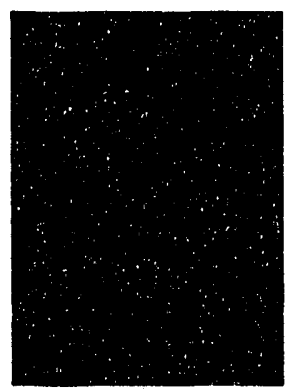

900

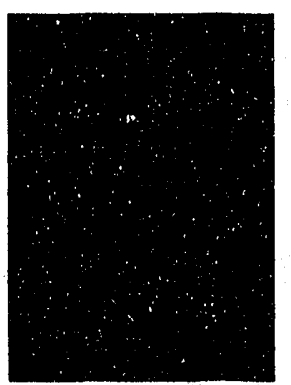

390

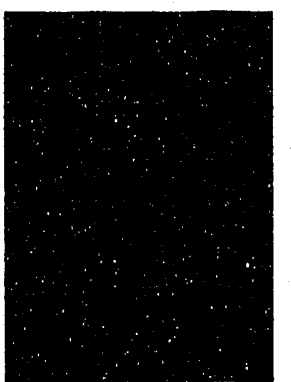

510

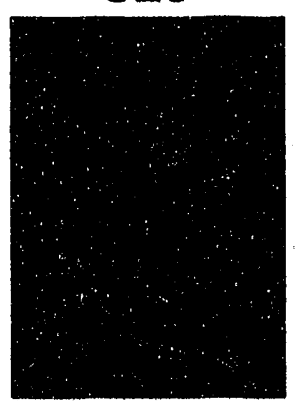

660

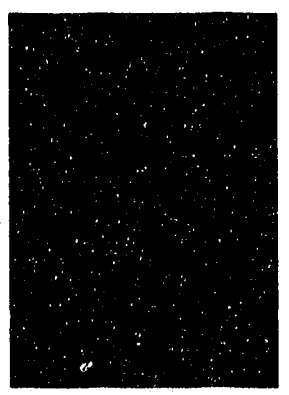

930

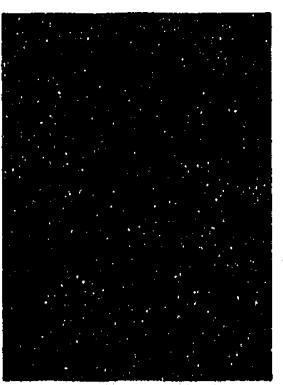

480

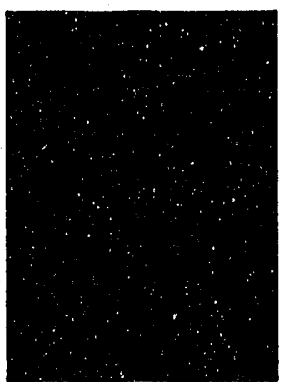

540

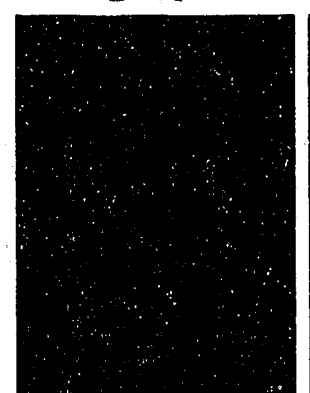

720

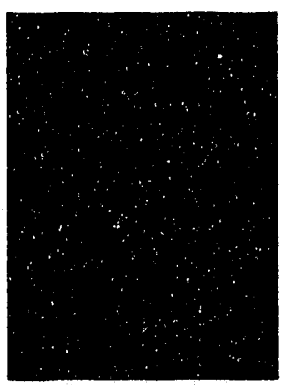

450

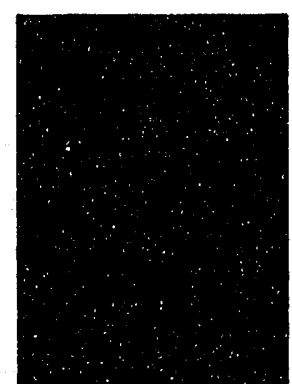

570

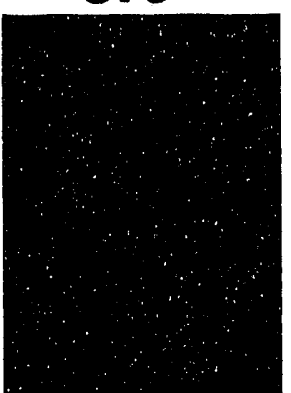

870

Figure 55b. Images of Ignition and Combustion Sequence with Boundary

$$
\text { Detection Function }(l=4.67 \mathrm{~mm})
$$



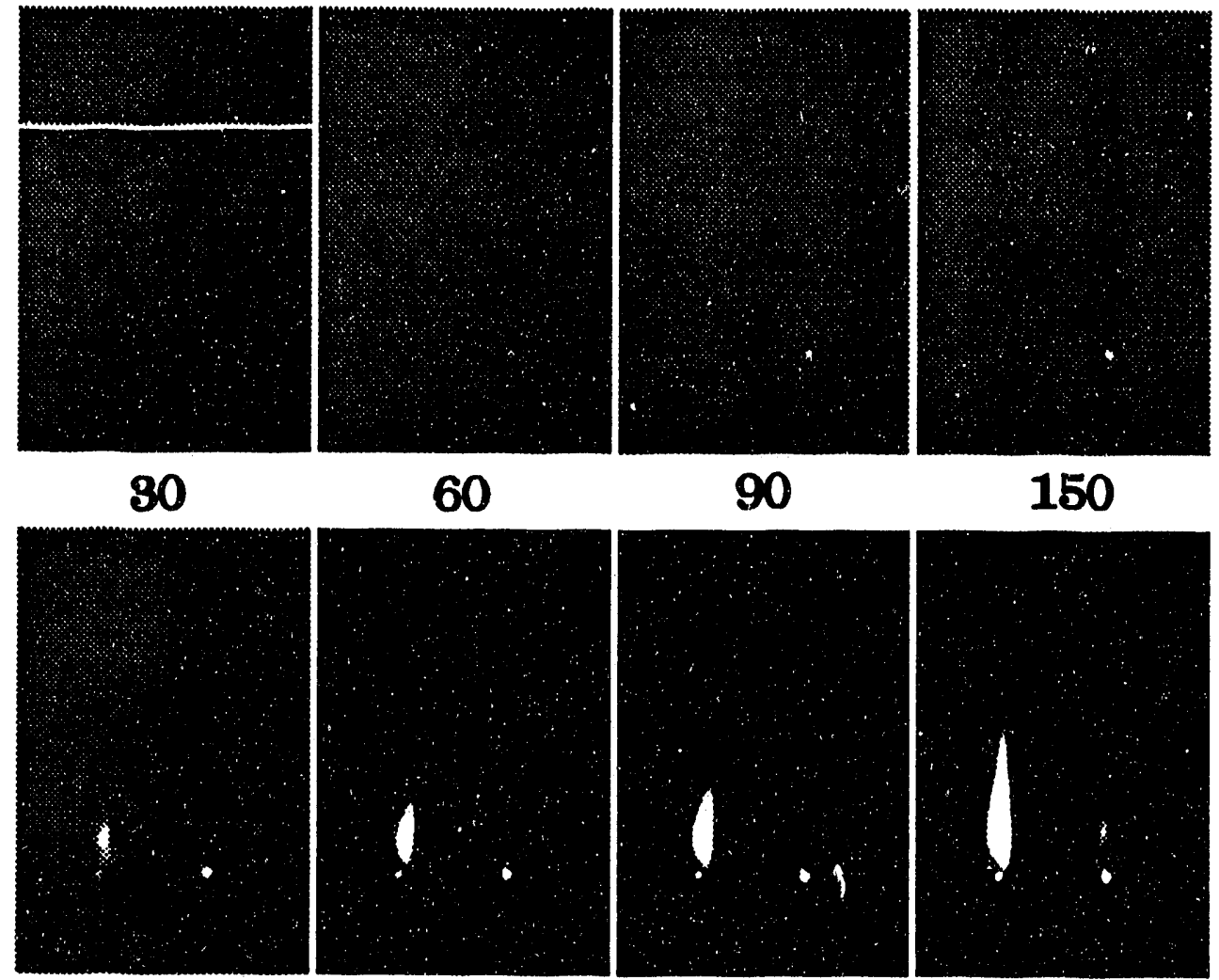

\section{0}

270

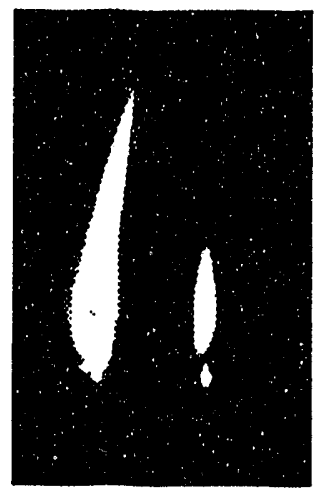

330

360

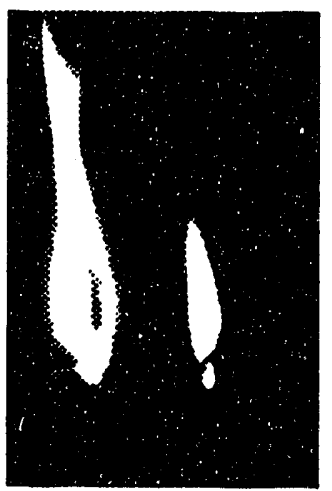

600

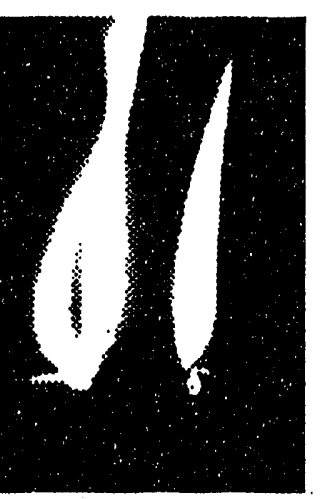

630

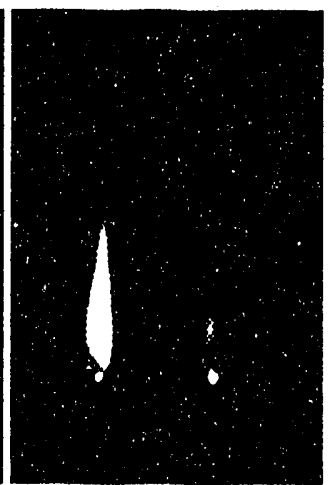

420

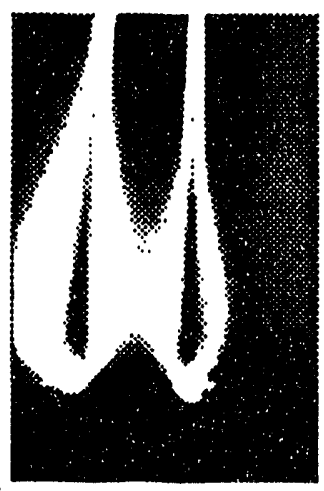

660

Figure 56a. Ignition and Combustion Sequence for Small High Volatile

Coal Two-Particle Array $(l=7.1 \mathrm{~mm})$ 


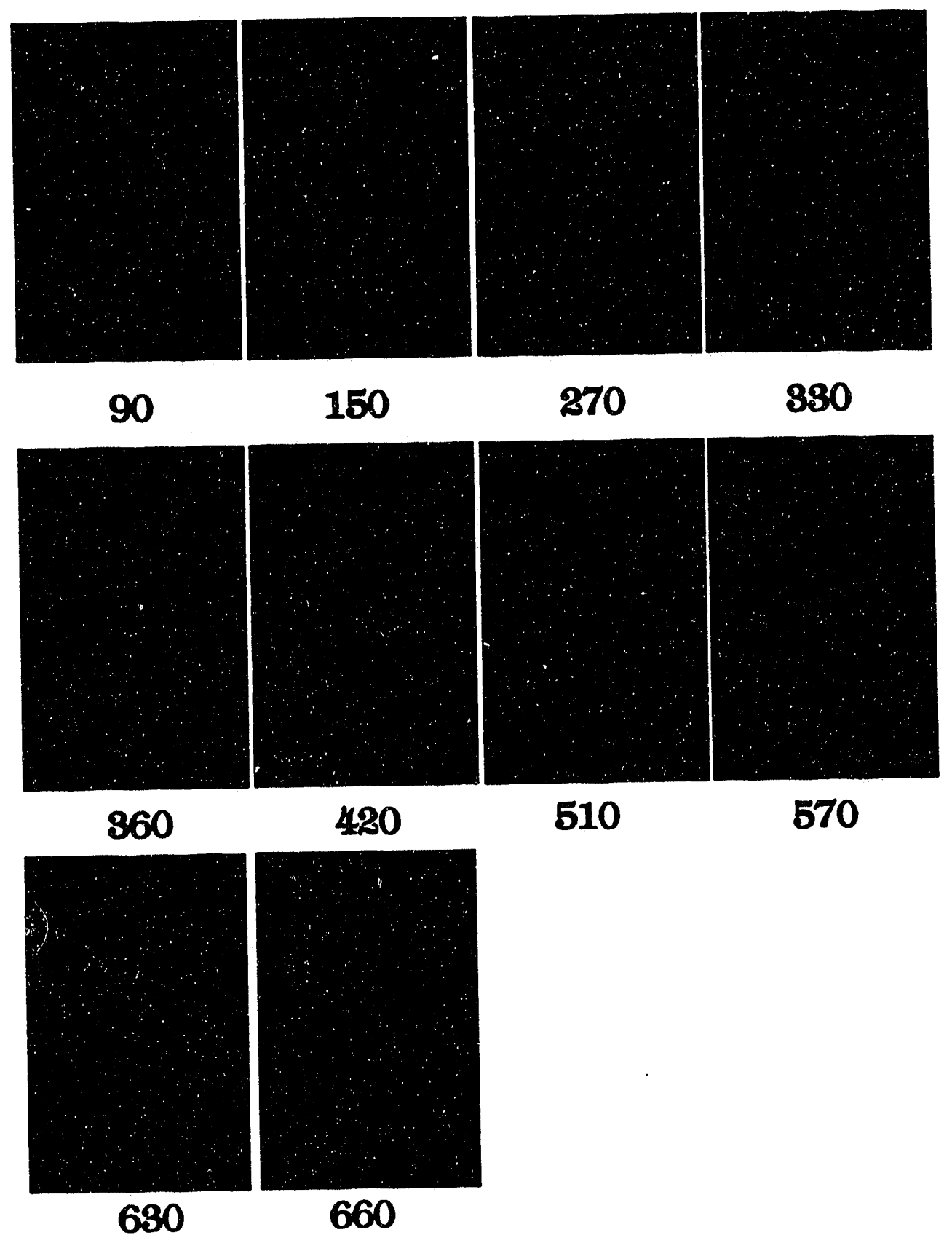

Figure 56b. Images of Ignition and Combustion Sequence with Boundary Detection Function $(l=7.1 \mathrm{~mm})$ 

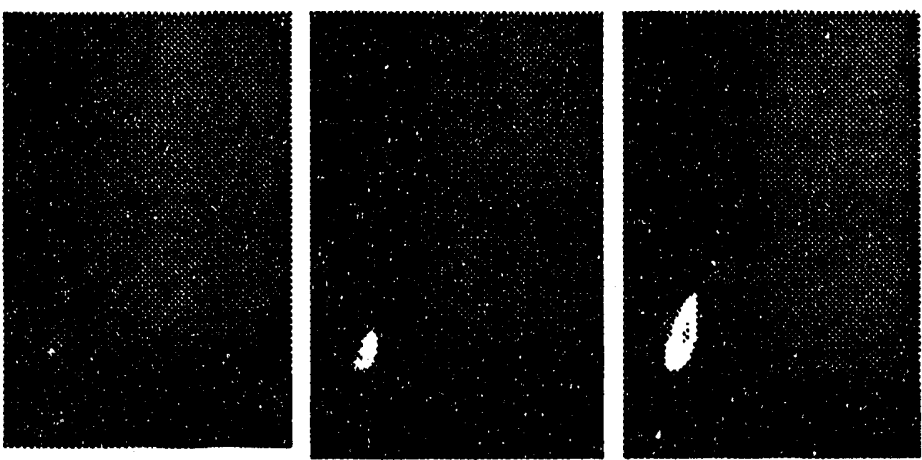

30

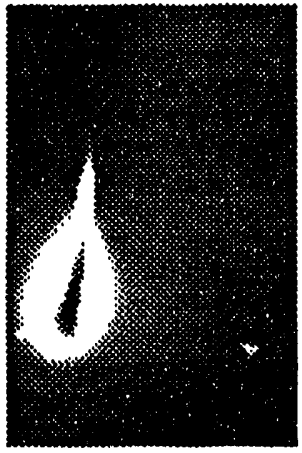

240

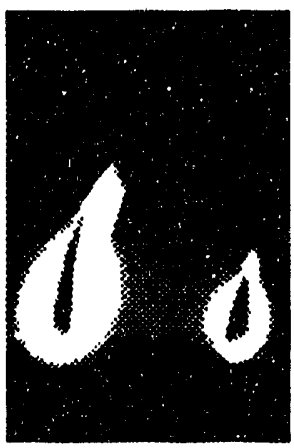

390
60

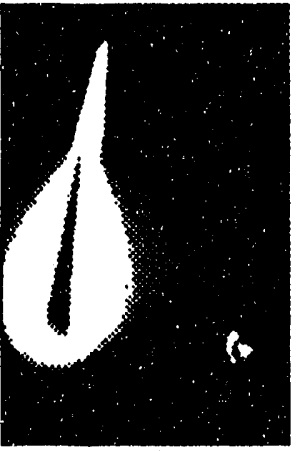

300

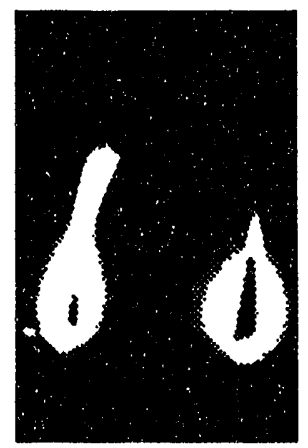

420
120

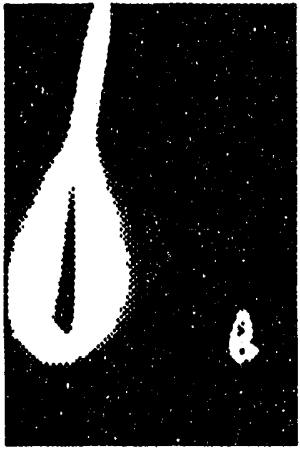

330

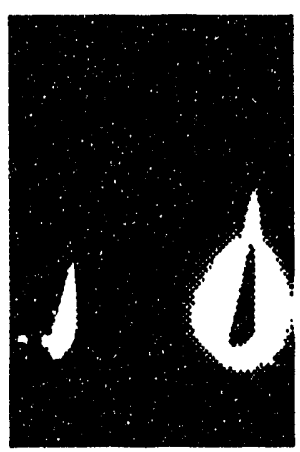

450

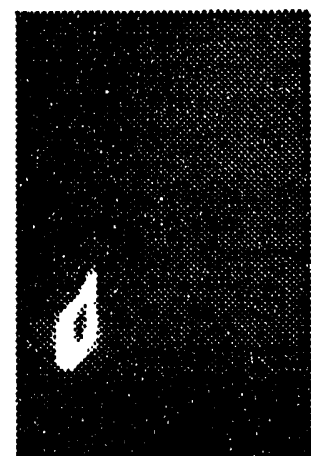

150

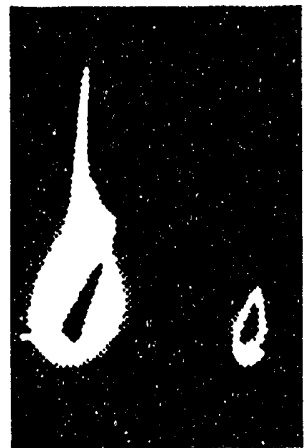

360

Figure 57a. Ignition and Combustion Sequence for Small High Volatile Coal Two-Particle Array $(l=10.16 \mathrm{~mm})$ 

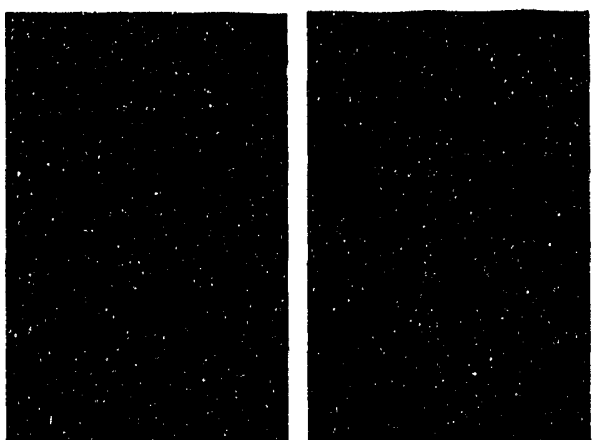

330

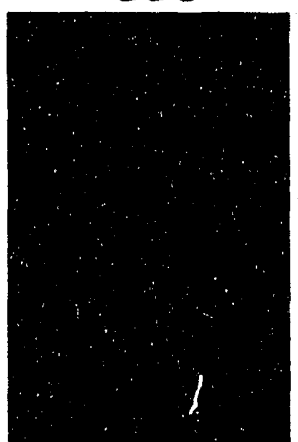

420

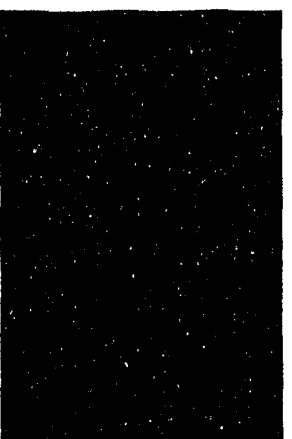

450

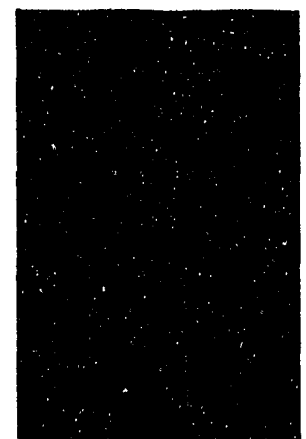

890

Figure 57b. Images of Ignition and Combustion Sequence with Boundary Detection Function $(l=10.16 \mathrm{~mm})$ 


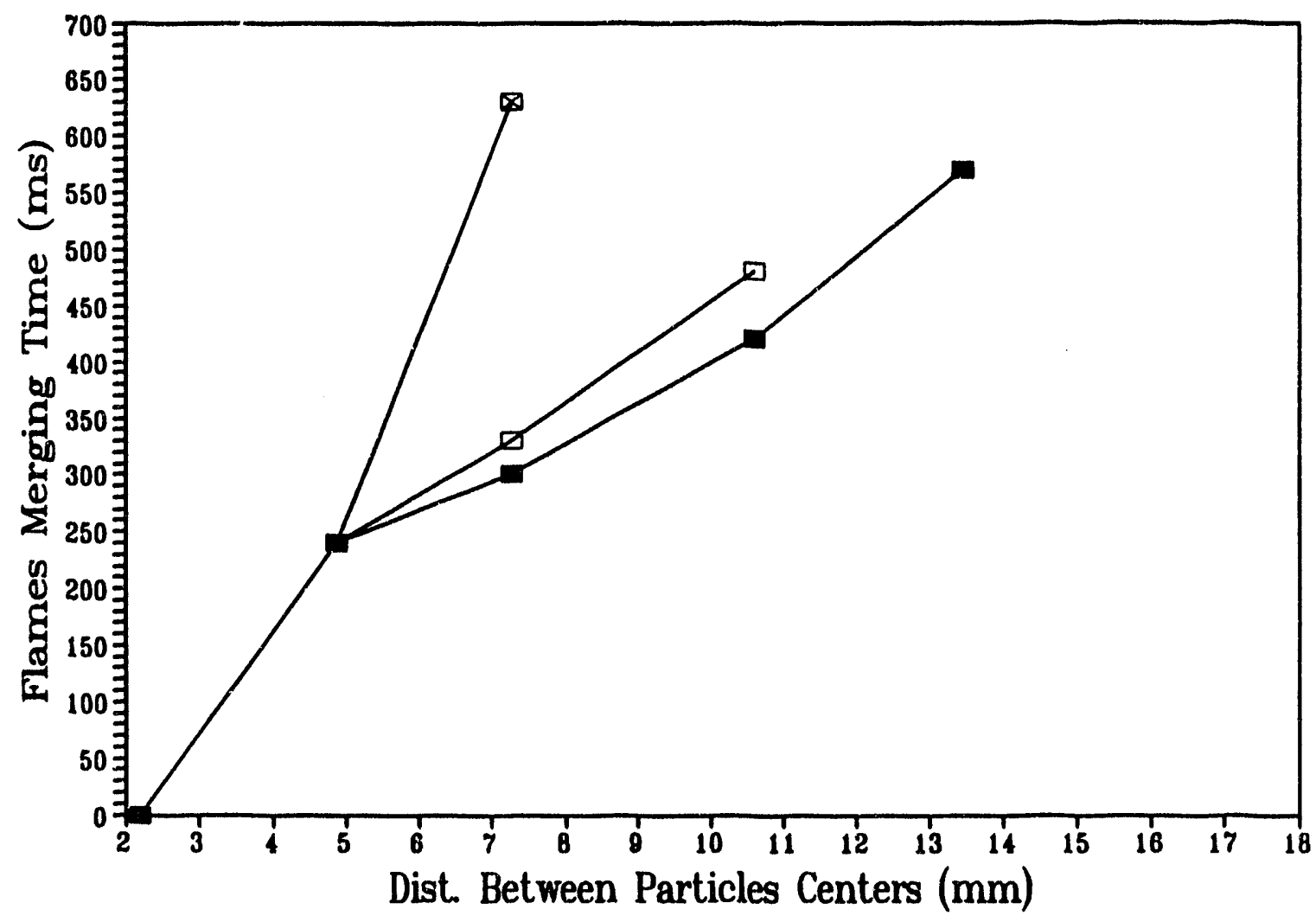

Large Particles - Intermed Particles Small Particles

Figure 58. Variation of Flames Merging Time with Interparticle Spacing for High volatile Coal Two-Particle Arrays 
noting. As the particle size increases, the flame merging time decreases at a specified interparticle spacing, since larger particles involve a larger flame. Also, as the particles sizes increase, the critical distance increases. For example, for the large particles, the flames do not merge beyond $15.88 \mathrm{~mm}$ while the corresponding distances are $13.4 \mathrm{~mm}$ and $10.16 \mathrm{~mm}$ for the intermediate and small particles respectively. In addition, at a distance of approximately $7.2 \mathrm{~mm}$, the flames of large particles merge at $300 \mathrm{~ms}$ while at $330 \mathrm{~ms}$ and at $630 \mathrm{~ms}$ for the intermediate and small particles respectively. Tables 8 through 10 summarize flame merging time as a function of the distance between the two particles for the three arrays.

\section{VI.5.1.2 Vertical Geometry}

In this experiment, two particles were placed vertically, one on top of the other. As they were exposed to the flat flame provided by the burner, the lower particle ignited first. Ignition, as observed previously (horizontal geometry), started at the particle's surface and volatile ejected afterwards. By that time, the ignition of the upper particle started, again on its surface. Ignition of both particles oecurred at the leading edge. As more time elapsed, volatile ejection rate increased allowing them to burn in the gas phase for both particles. The flame of each particle propagated and grew larger as more time elapsed. Eventually, both flames merged together forming a single larger flame (Figure 59).

\section{VI.5.2 Medium Volatile Coal (BSOC 1336)}

Ignition and combustion characteristics of this coal array resemble those obtained 
Table 8. Combustion Characteristics of Large High Volatile Coal Two-Particle Arrays

\begin{tabular}{|c|c|c|c|c|}
\hline $\begin{array}{l}\text { Dictance Bet } \\
\text { Centers of Particles }\end{array}$ & Tgintion & 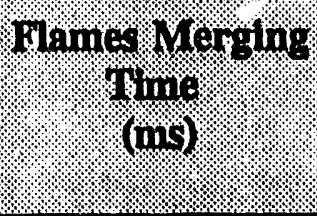 & $=9$ & did \\
\hline 2.20 & 150 & Immediately & 1.02 & 0.98 \\
\hline 4.86 & 270 & 240 & 1.0 & 1.02 \\
\hline 7.26 & 150 & 300 & 0.99 & 1.01 \\
\hline 10.60 & 180 & 420 & 0.98 & 0.96 \\
\hline 13.45 & 90 & 570 & 1.01 & 1.03 \\
\hline 15.88 & 120 & No Merging & 0.98 & 1.0 \\
\hline
\end{tabular}

Table 9. Combustion Characteristics of Intermediate High Volatile Coal Two-Particle Arrays

\begin{tabular}{|c|c|c|c|c|}
\hline 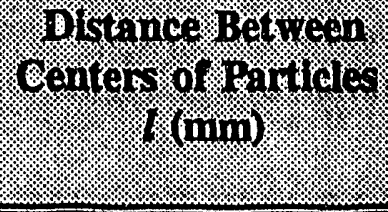 & I & 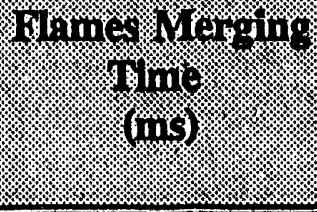 & \multicolumn{2}{|c|}{ 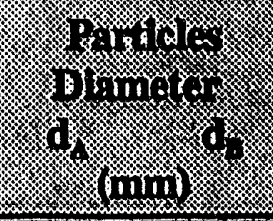 } \\
\hline 2.13 & 210 & Immediately & 0.82 & 0.79 \\
\hline 4.53 & 150 & 240 & 0.80 & 0.82 \\
\hline 7.12 & 60 & 330 & 0.78 & 0.80 \\
\hline 10.34 & 60 & 480 & 0.77 & 0.78 \\
\hline 13.42 & 270 & No Merging & 0.82 & 0.80 \\
\hline
\end{tabular}


Table 10. Combustion Characteristics of Small High Volatile

Coal Two-Particle Arrays

\begin{tabular}{|c|c|c|c|c|}
\hline 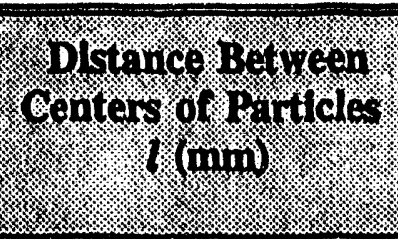 & 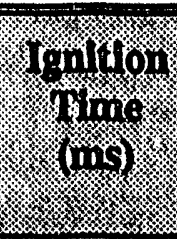 & Merging thime & 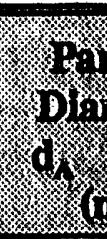 & $\left(\frac{1}{6}\right.$ \\
\hline 2.05 & 300 & Immediately & 0.57 & 0.59 \\
\hline 4.43 & 330 & 240 & 0.58 & 0.60 \\
\hline 7.10 & 60 & 630 & 0.59 & 0.60 \\
\hline 10.16 & 30 & No Merging & 0.61 & 0.62 \\
\hline
\end{tabular}




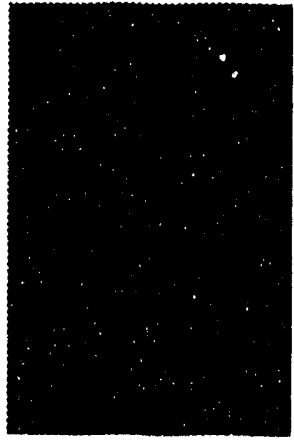

30

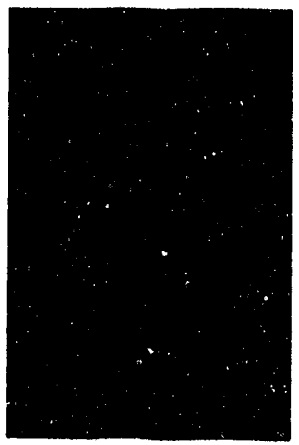

600

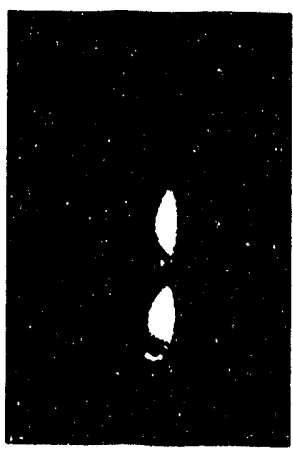

690

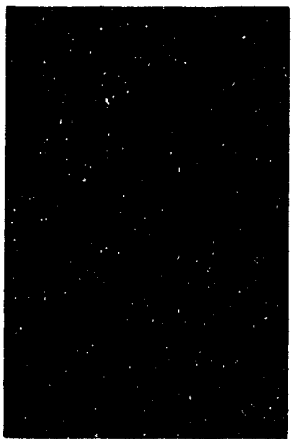

880

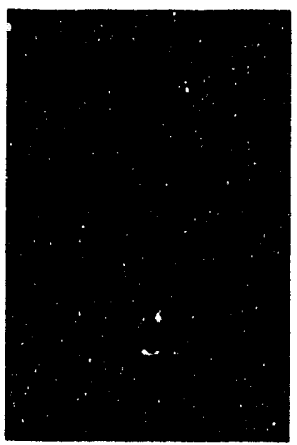

680

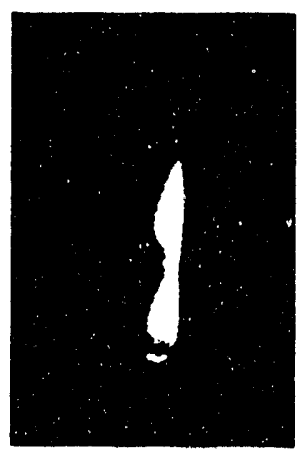

720

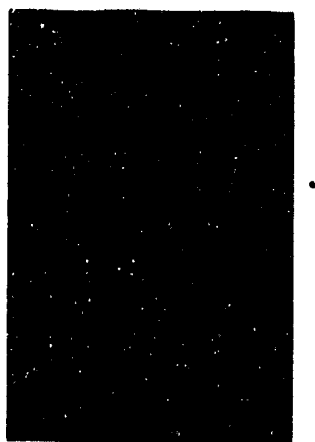

860

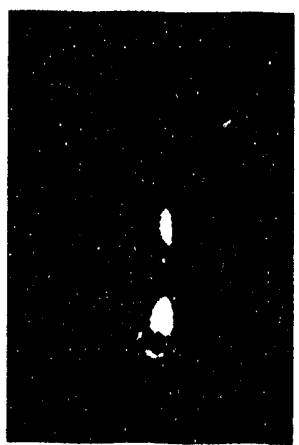

660

Figure 59a. Ignition and Combustion Sequence for High Volatile Two-Particle Array with Vertical Geometry 


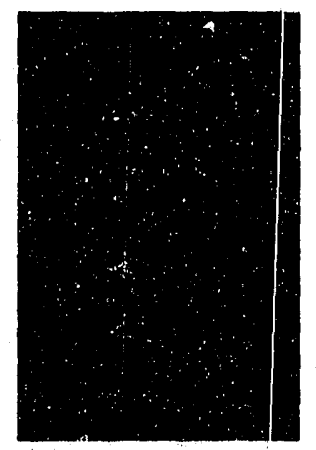

630

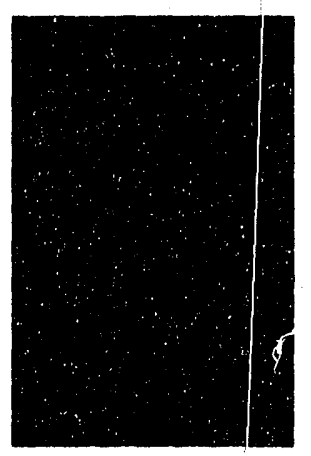

690

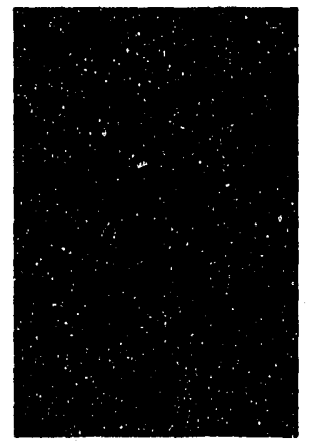

660

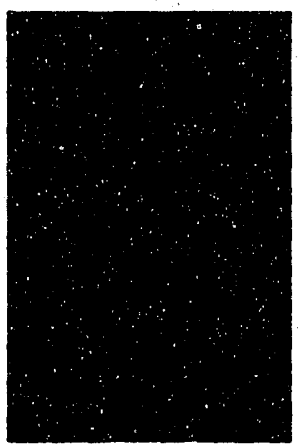

720

Figure 59b. Images of Ignition and Combustion Sequence With Boundary Detection Function (Vertical Geometry) 
for the high volatile coal. Ignition was observed to start at the particles surface (leading edge), and individual flames were formed due to the burning of ejected volatile in the gas phase. Upon formation, both flames merged together forming one common flame around the two particles. Ignition and combustion processes are illustrated in Figures 60 to 65. Flames merging times expressed a strong relationship with the distance between the two particles (Figure 66). As distance increased, flames merging time increased as observed with previous coal. Also, the direction of flames depends on the shape of the particles.

It is found from Figure 67 that flames do not merge beyond $17.98 \mathrm{~mm}$ for the medium volatile coal, while flames do not merge beyond $15.88 \mathrm{~mm}, 13.42 \mathrm{~mm}, 10.16$ $\mathrm{m}, \mathrm{m}$, for the high volatile large, intermediate and small particles respectively. Table 11 translates the graphical form of the results into tabulated data.

\section{VI.6 Char Two-Particle Array}

A strong analogy exists between the ignition and combustion characteristics of an isolated char particle and a two-particle array. Ignition started at the surface of the particles. Combustion of fixed carbon in the char progressed to include larger portions of the particles, as more time elapsed and as combustion intensified, until a complete comoustion was reached, a behavior which was observed to occur previousiy with the isolated char particle combustion. In addition, both char particles were expected to form a common contour upon burning, instead each particle burned individually (Figure 68). 


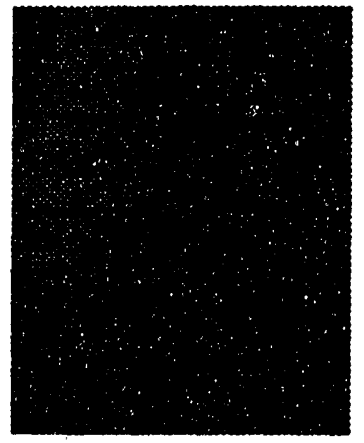

30

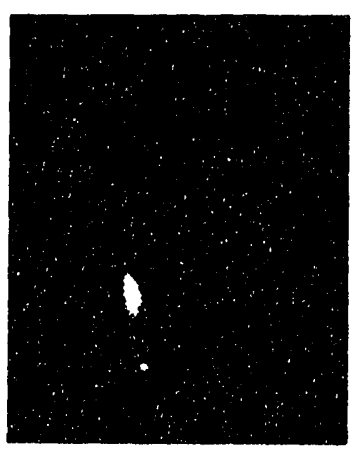

150

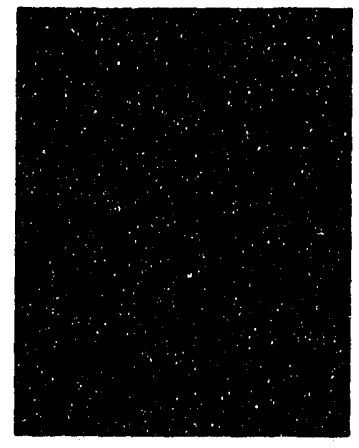

60

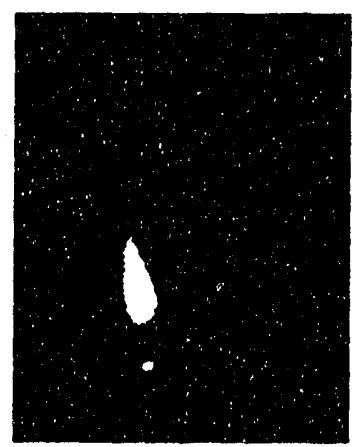

210

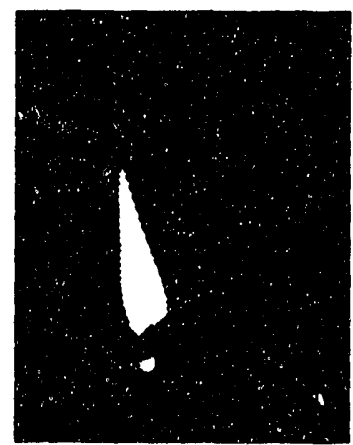

270

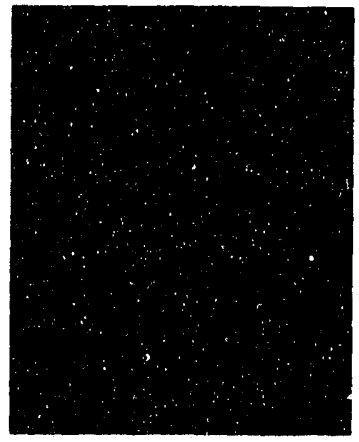

120

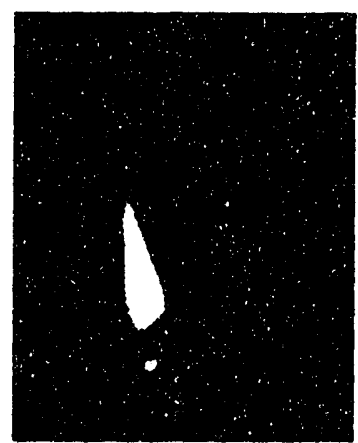

340

Figure 60a. Ignition and Combustion Sequence for Medium Volatile Coal Two-Particle Array $(l=2.54 \mathrm{~mm})$, Time in msec 


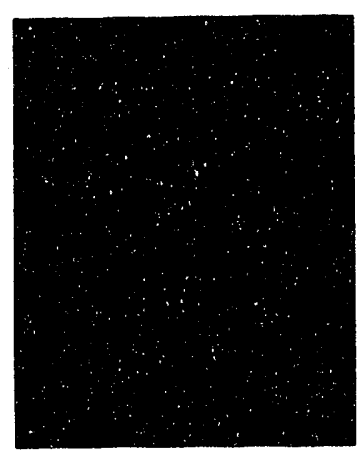

120

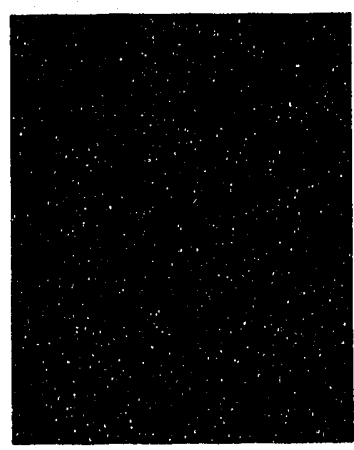

240

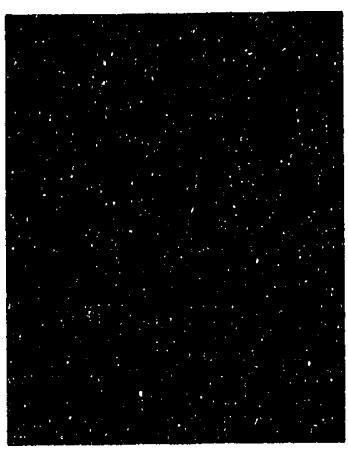

150

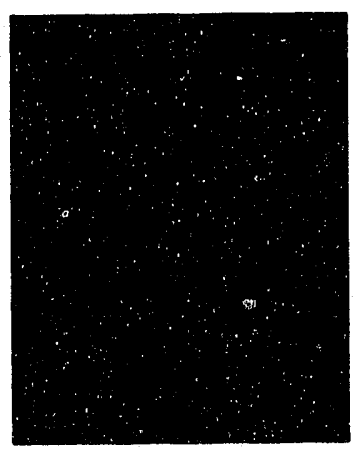

$\mathbf{2 7 0}$

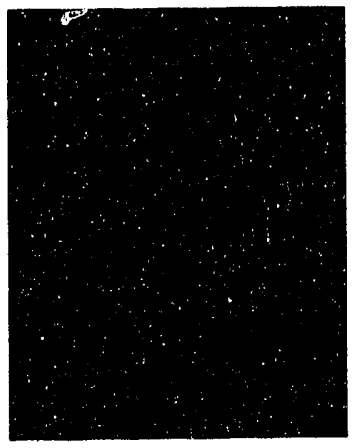

210

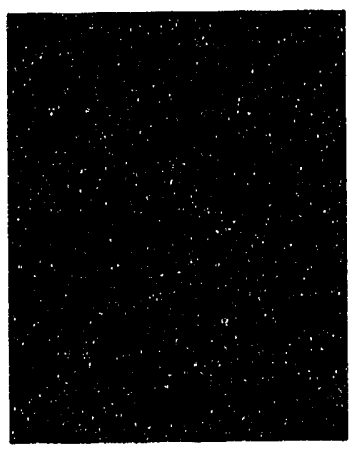

$\mathbf{3 0 0}$

Figure 60b. Images of Ignition and Combustion Sequence with Boundary Detection Function $(l=2.54 \mathrm{~mm})$ 


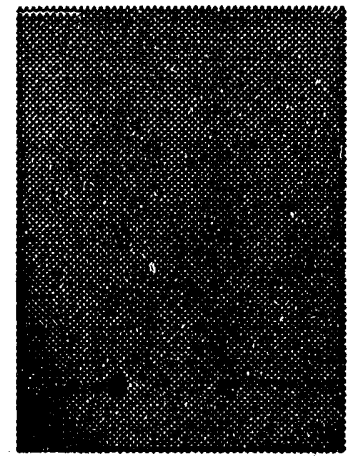

300

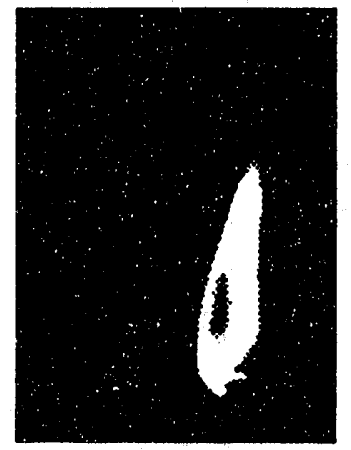

480

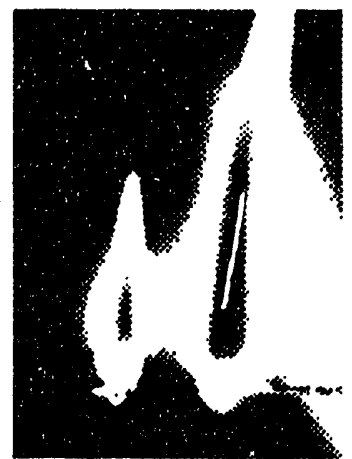

600

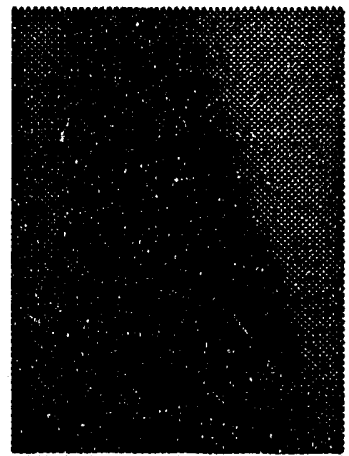

360

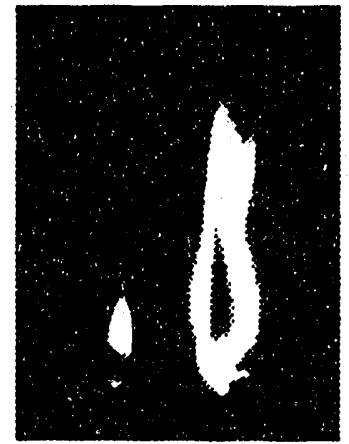

510

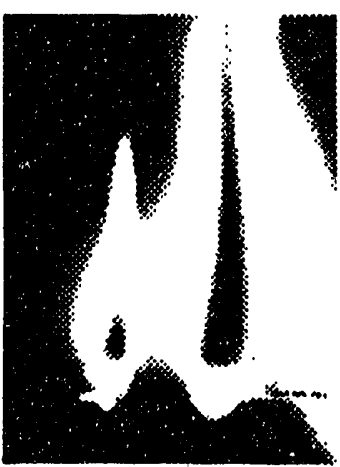

630

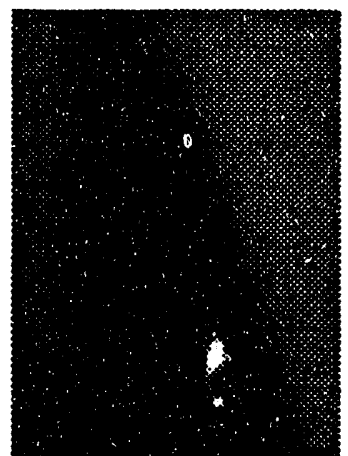

420

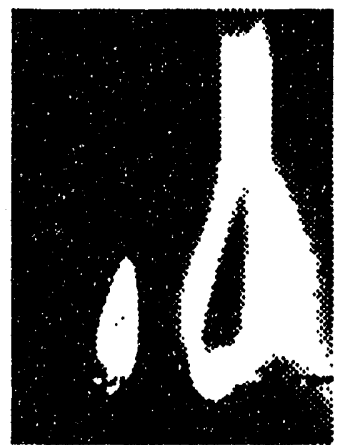

570

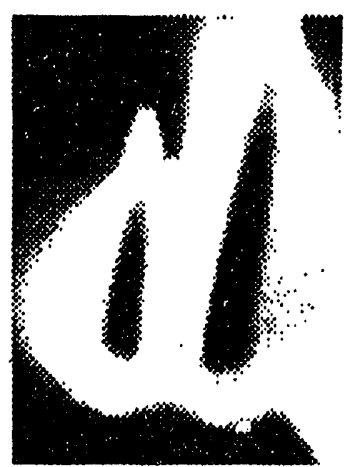

690

Figure 61a. Ignition and Combustion Sequence for Medium Volatile

Coal Two-Particle Array $(l=4.67 \mathrm{~mm})$ 


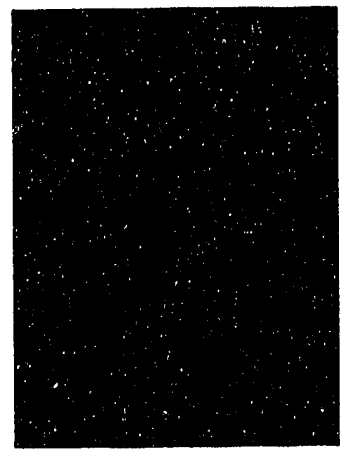

480

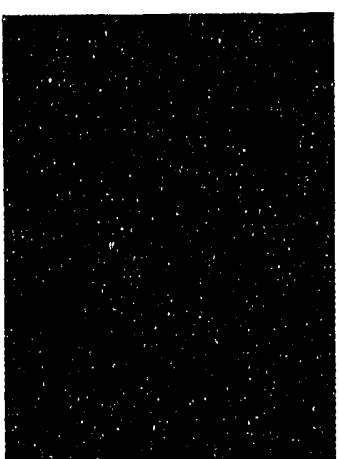

600

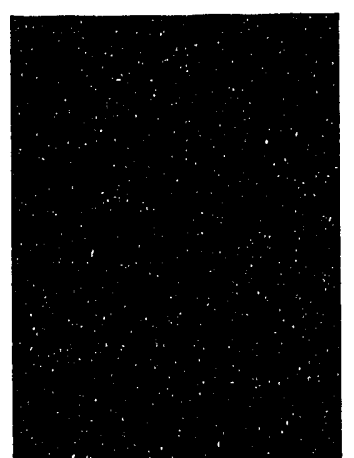

510

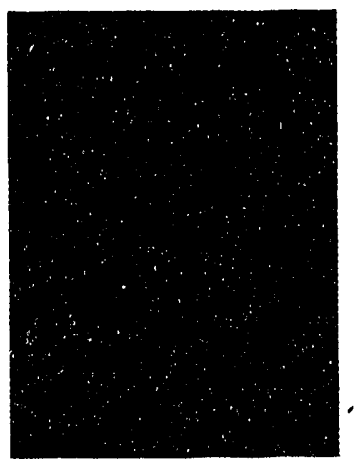

630

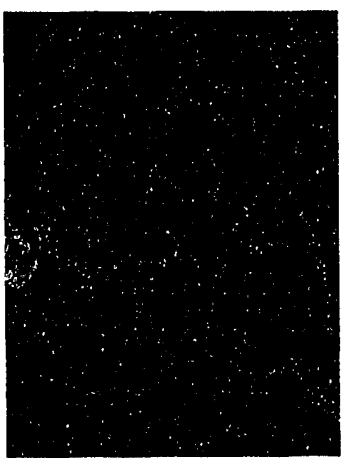

570

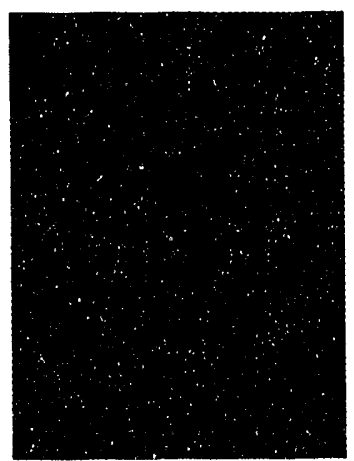

690

Figure 61b. Images of Ignition and Combustion Sequence with Boundary Detection Function $(l=4.67)$ 

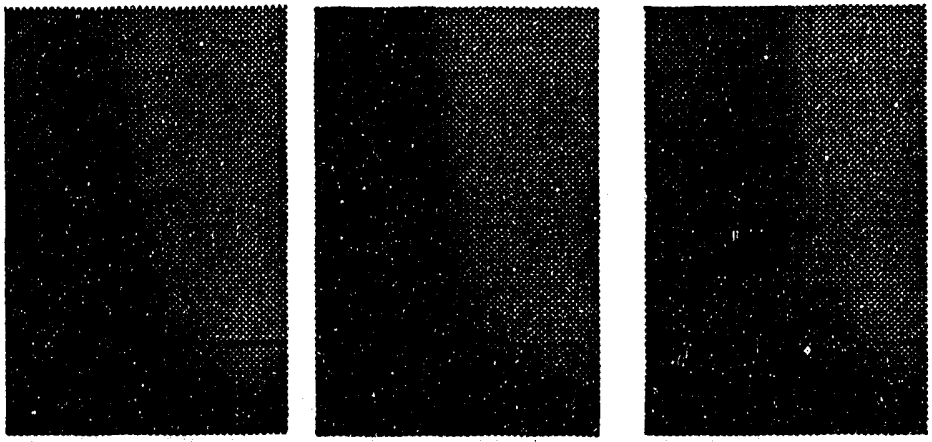

30

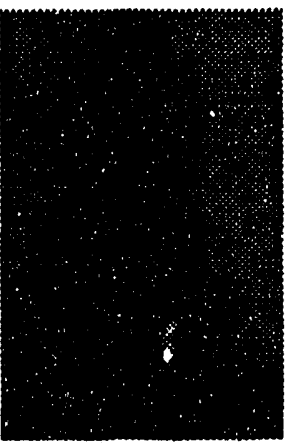

180

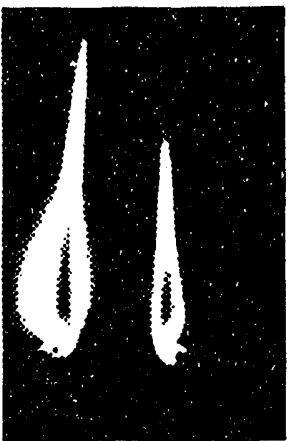

360
60

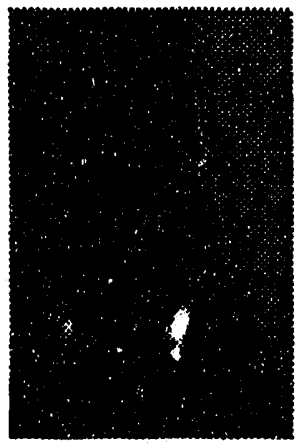

210

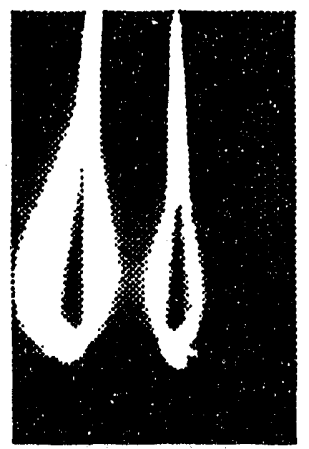

420
120

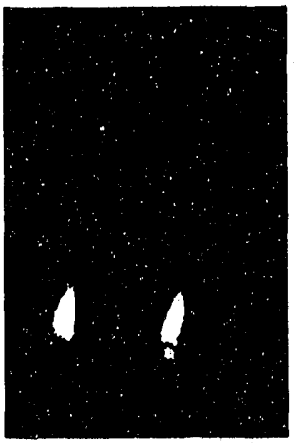

240

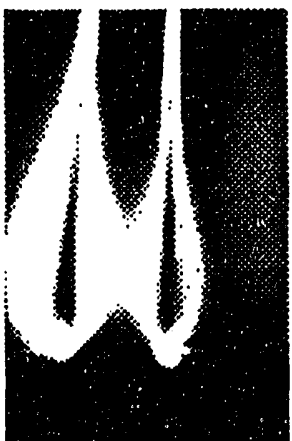

450

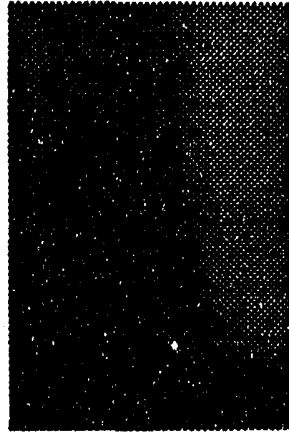

150

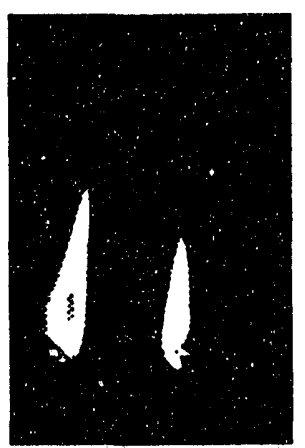

300

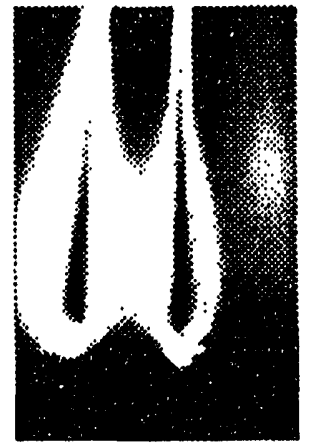

480

Figure 62a. Ignition and Combustion Sequence for Medium Volatile Coal Two-Particle Array $(l=7.85 \mathrm{~mm})$ 

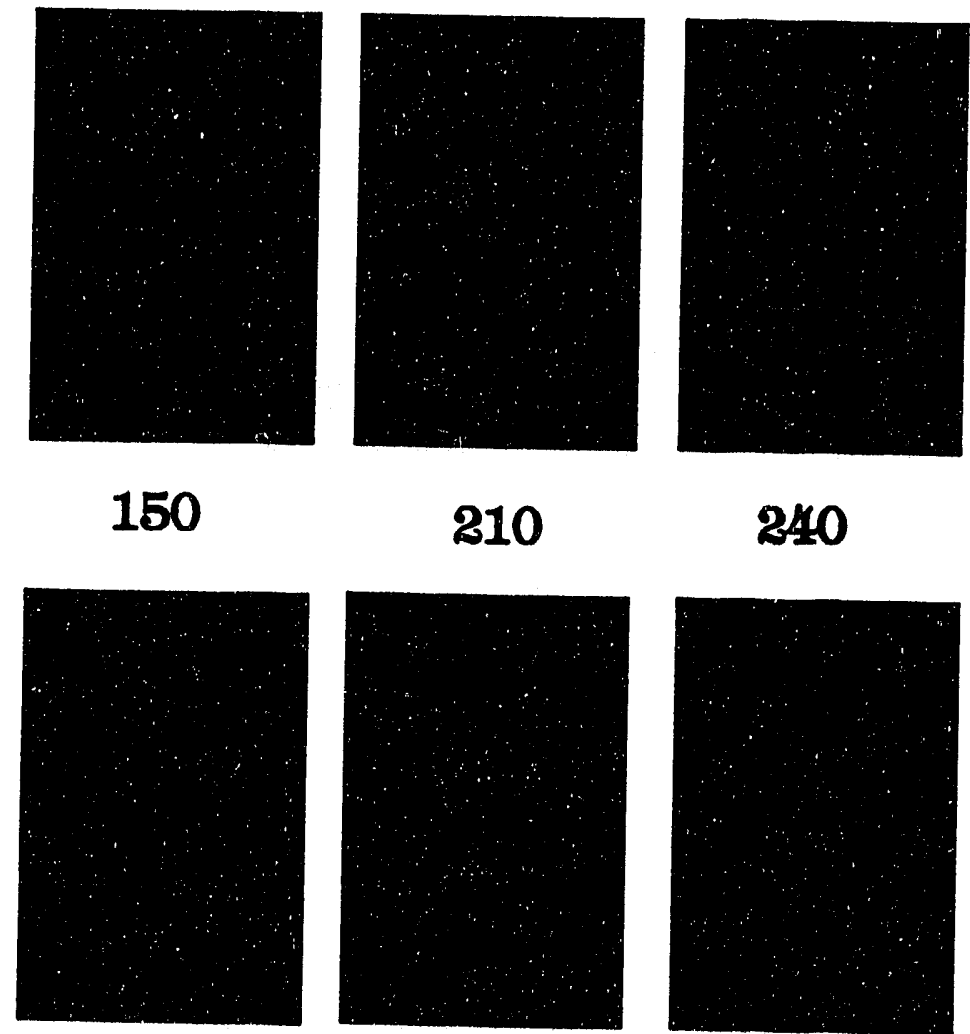

360

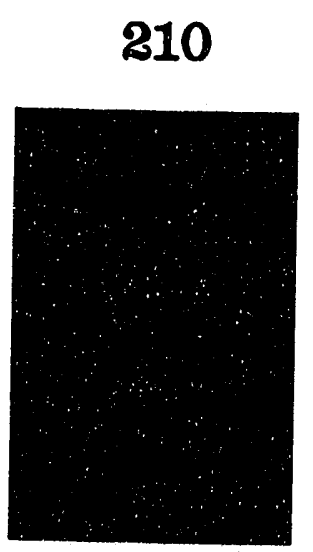

420
240

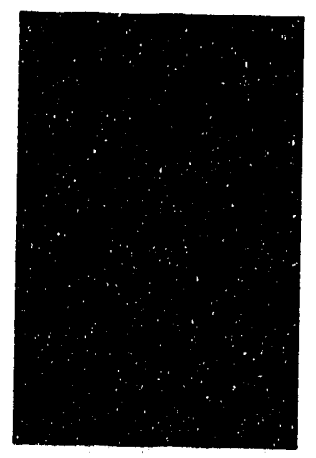

450

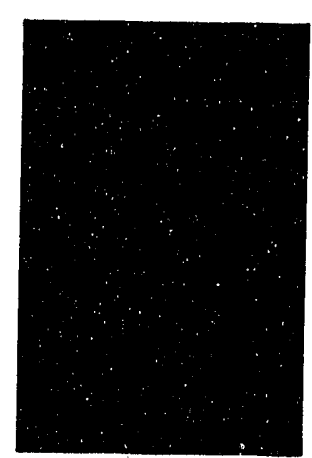

300

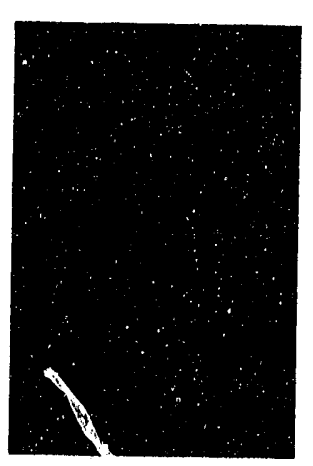

480

Figure 62b. Images of Ignition and Combustion Sequence with Boundary

Detection Function $(l=7.85 \mathrm{~mm})$ 


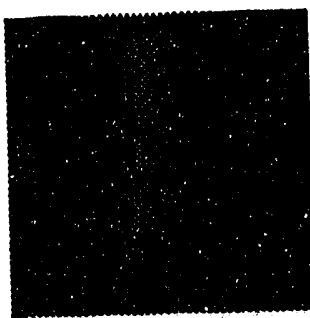

30

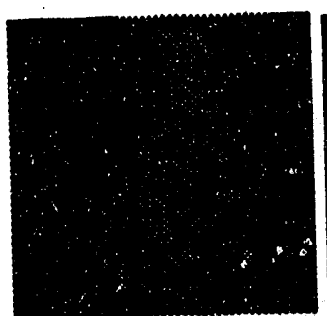

150

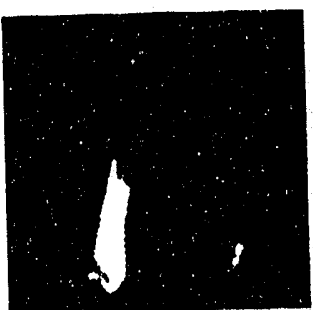

270

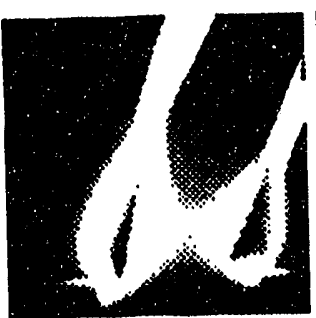

540

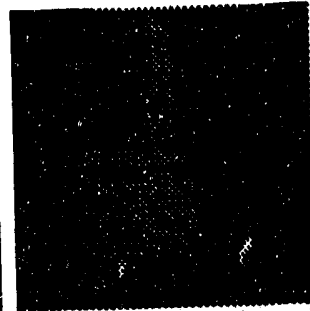

60

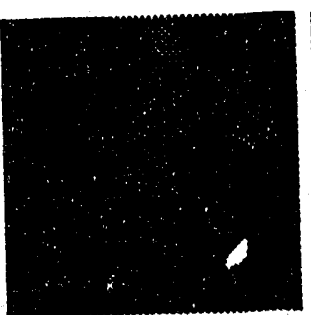

180

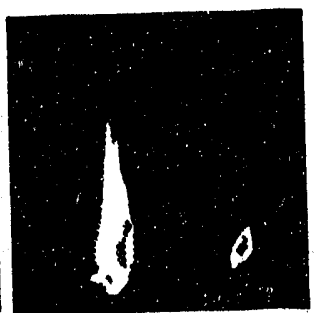

300

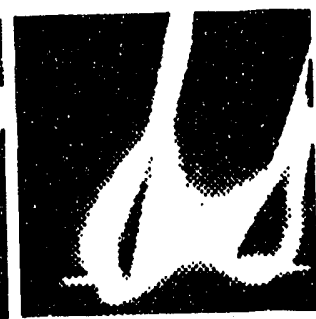

570

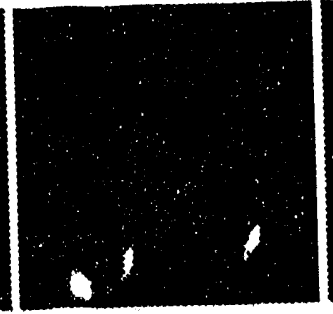

90

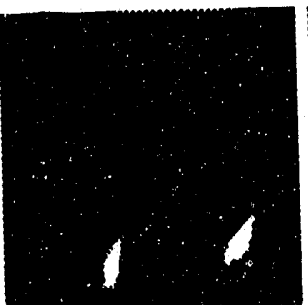

210

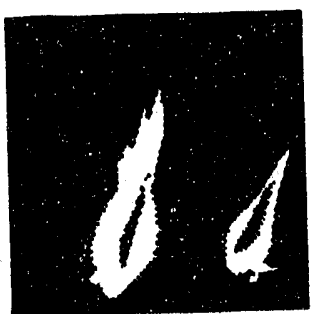

830

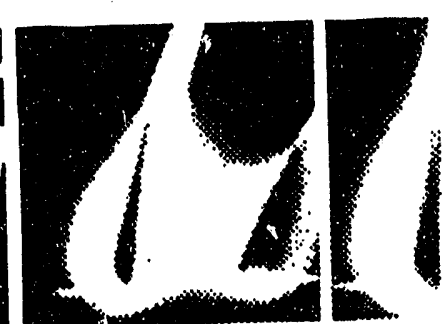

600

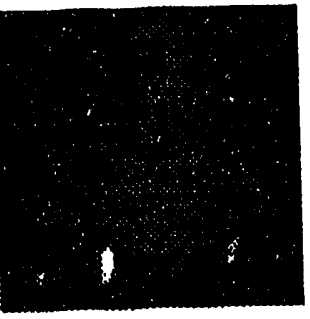

120

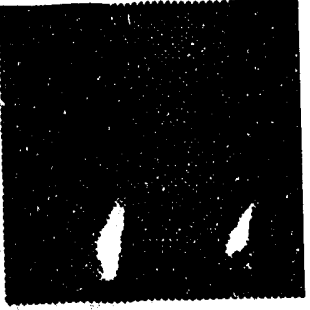

240

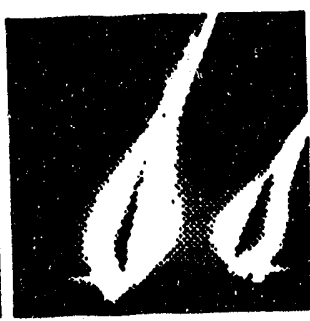

450

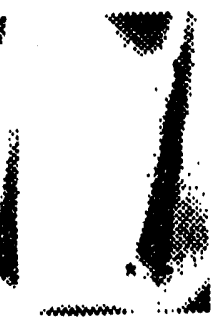

780

Figure 63a. Ignition and Combustion Sequence for Medium Volatile Coal Two-Particle Array $(l=13.58 \mathrm{~mm})$ 


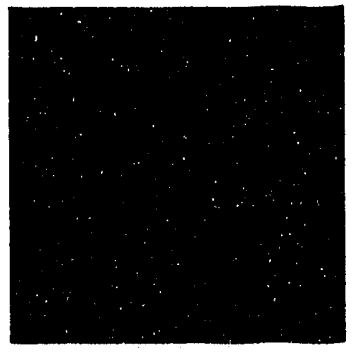

210

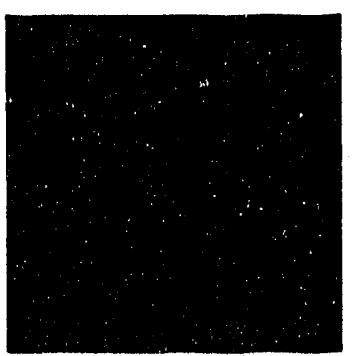

300

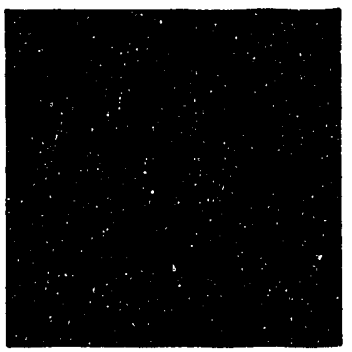

570

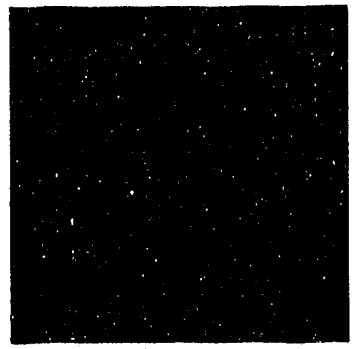

240

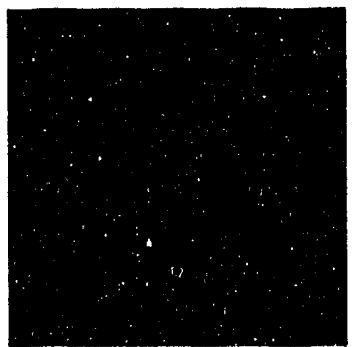

330

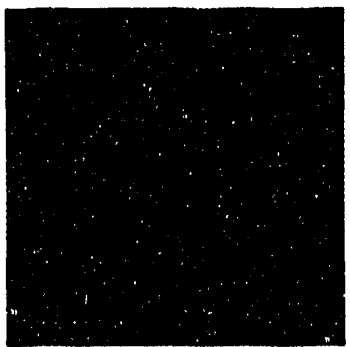

600

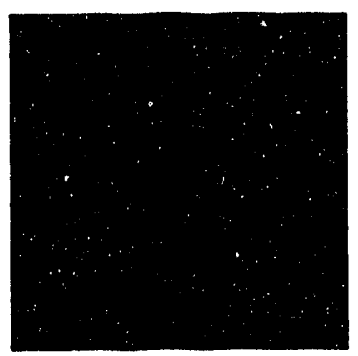

270

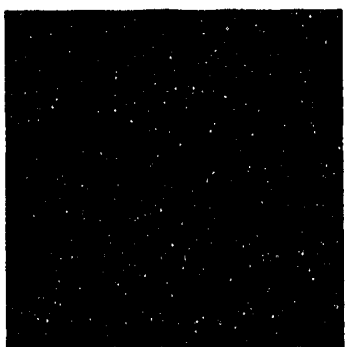

450

Figure 63b. Images of Ignition and Combustion Sequence with Boundary Detection Function $(l=13.58 \mathrm{~mm})$ 


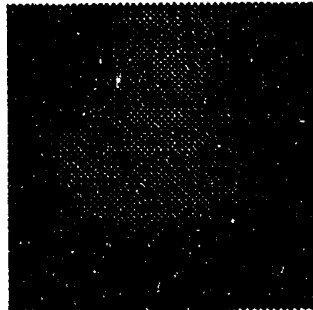

180

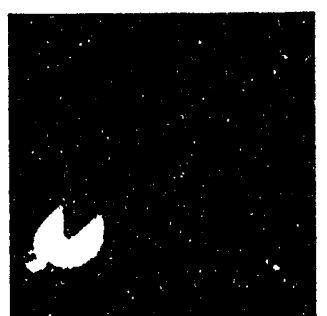

800

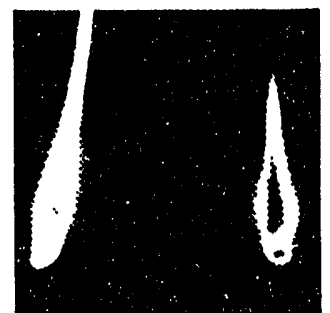

480

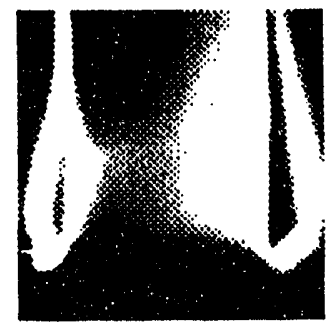

680

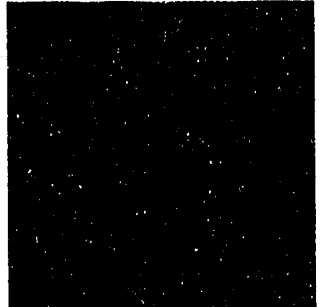

120

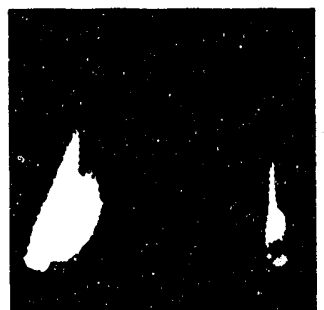

830

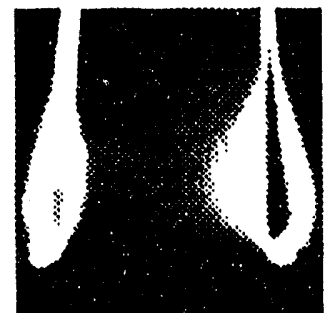

540

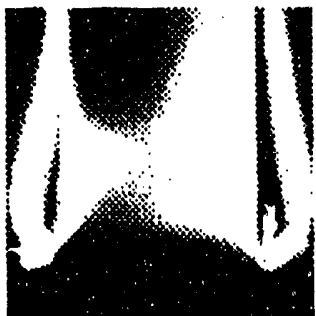

690

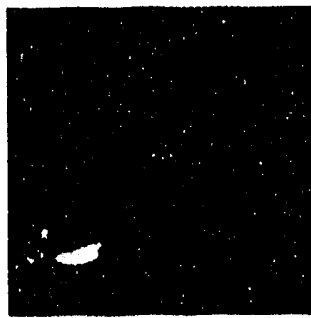

$\mathbf{8 7 0}$

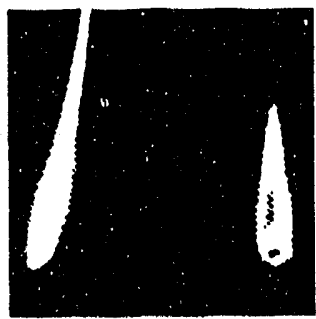

450

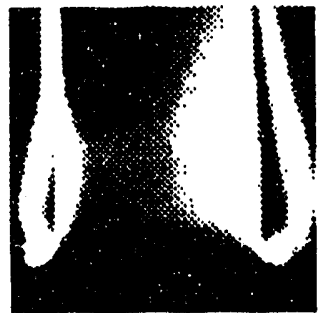

600

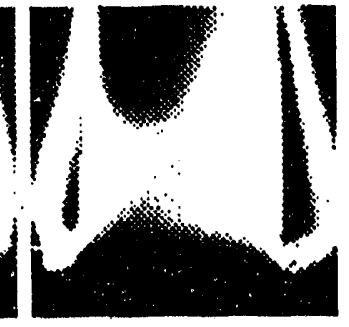

720

Figure 64a. Ignition and Combustion Sequence for Medium Volatile Coal Two-Particle Array $(l=15.87 \mathrm{~mm})$ 


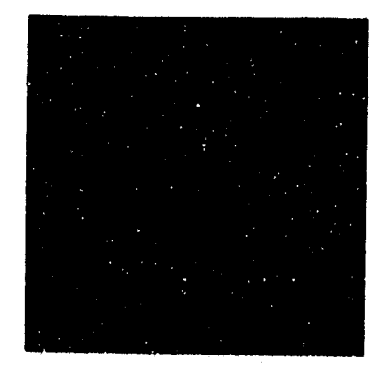

870

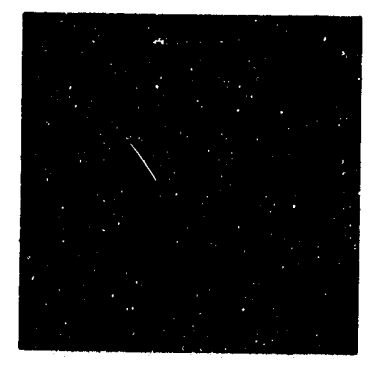

450

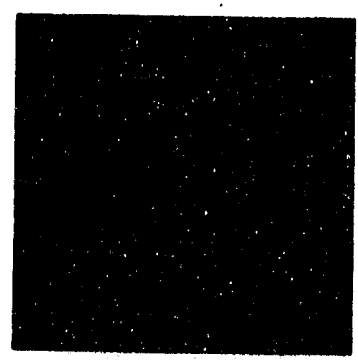

600

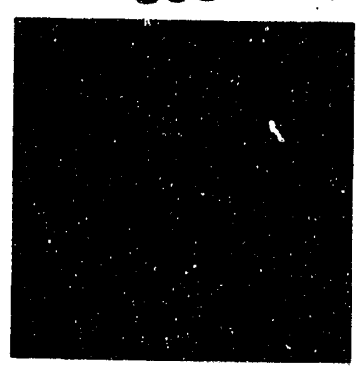

720

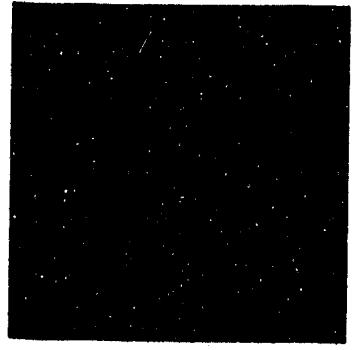

800

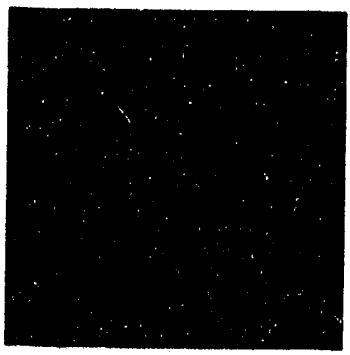

480

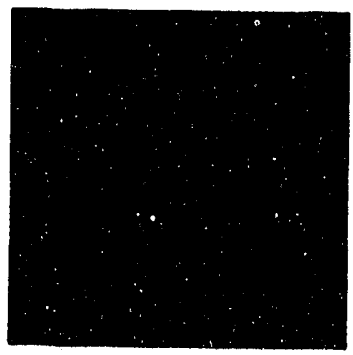

680

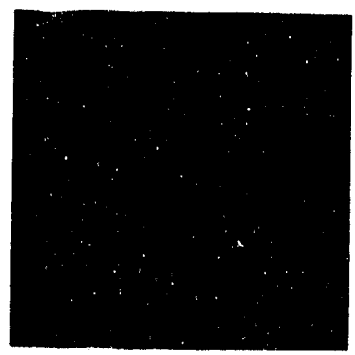

830

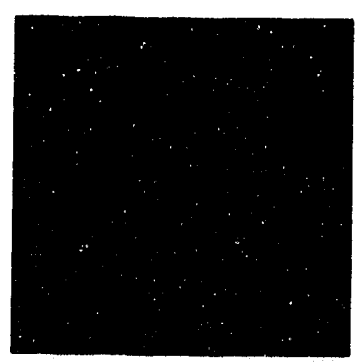

540

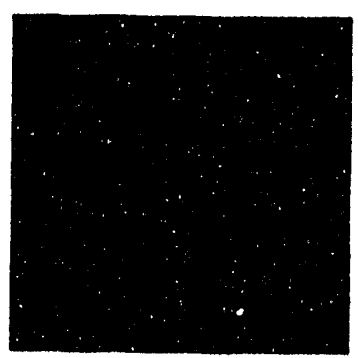

690

Figure 64b. Images of Ignition and Combustion Sequence with Boundary Detection Function $(l=15.87 \mathrm{~mm})$ 


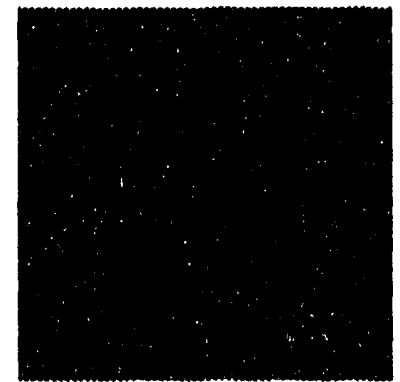

80

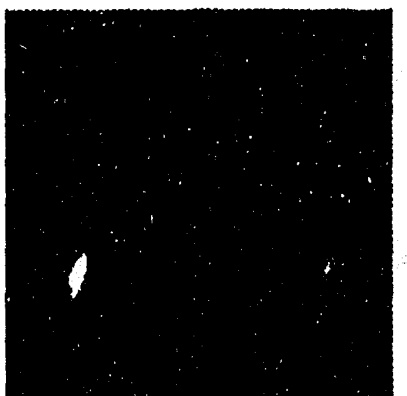

180

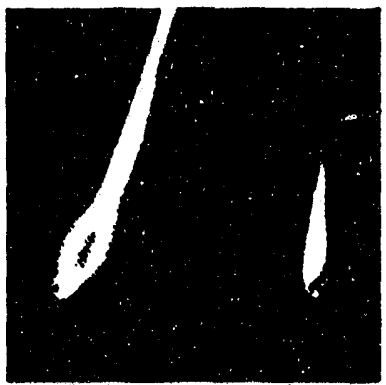

830

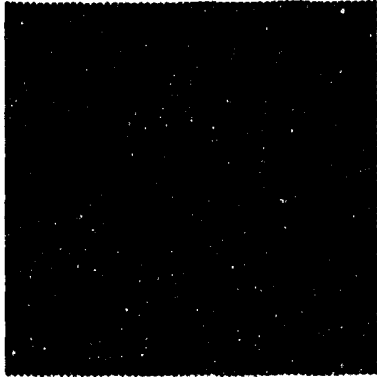

180

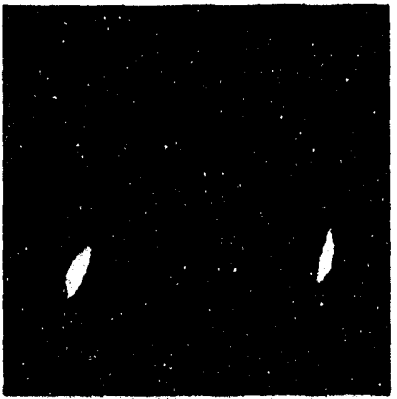

810

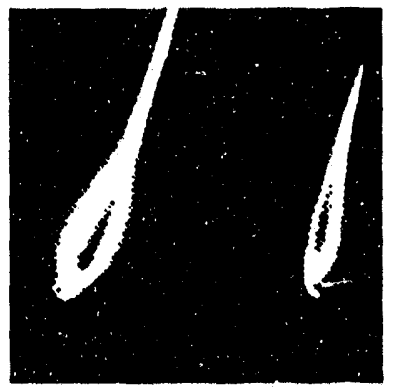

390

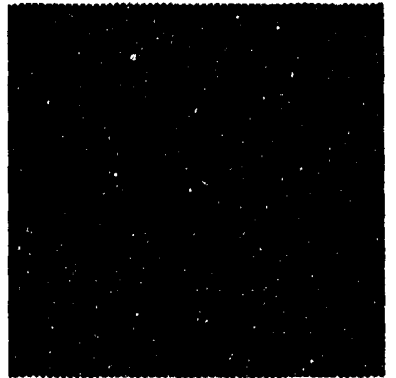

150

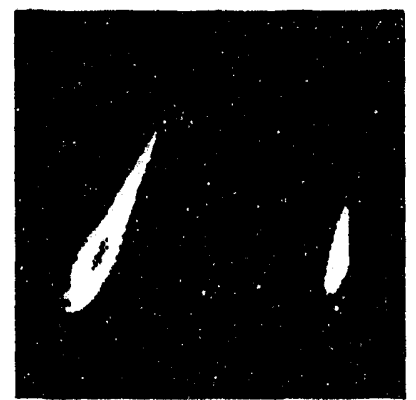

870

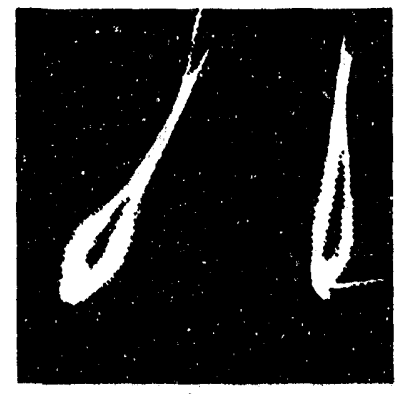

450

Figure 65a. Ignition and Combustion Sequence for Medium Volatile Coal Two-Particle Array $(l=17.98 \mathrm{~mm})$ 


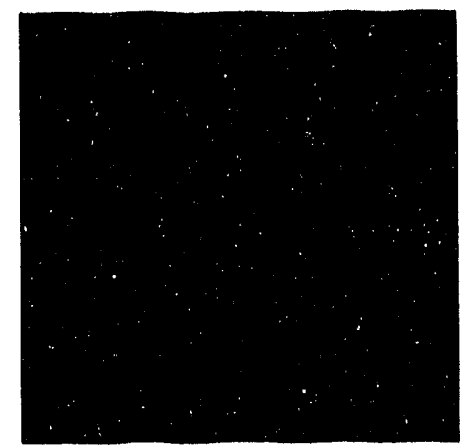

210

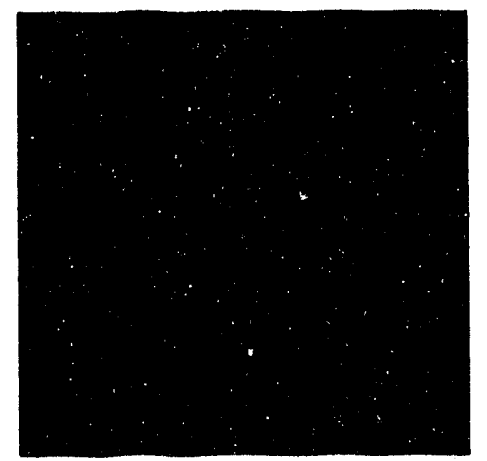

390

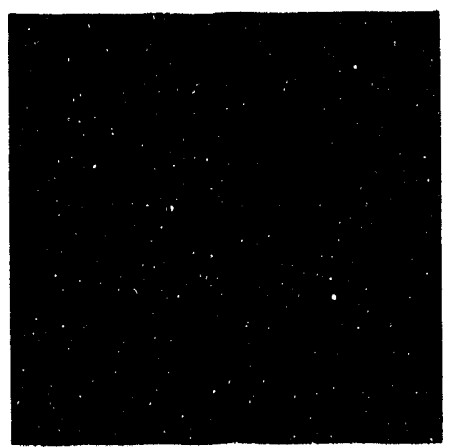

270

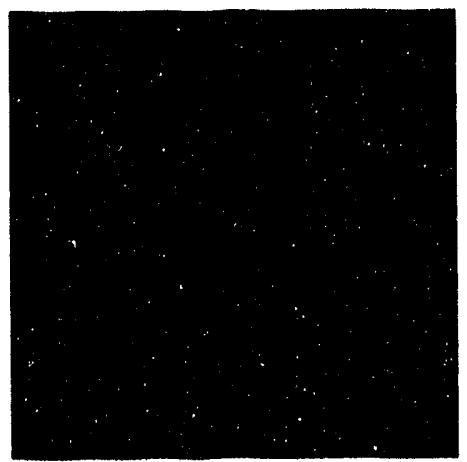

450

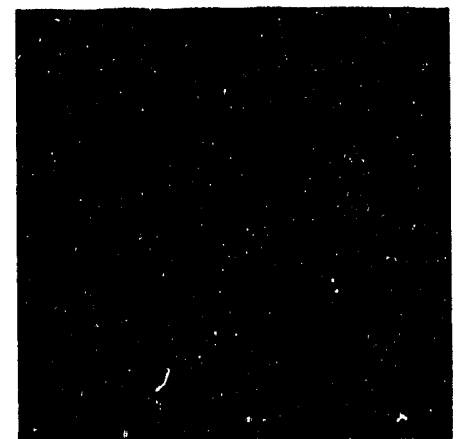

330

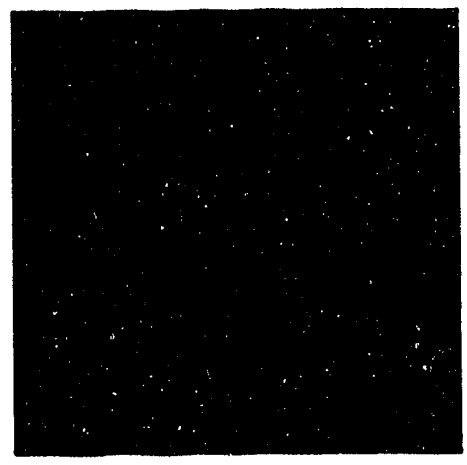

480

Figure 65b. Images of Ignition and Combustion Sequence with Boundary Detection Function $(l=17.98 \mathrm{~mm})$ 


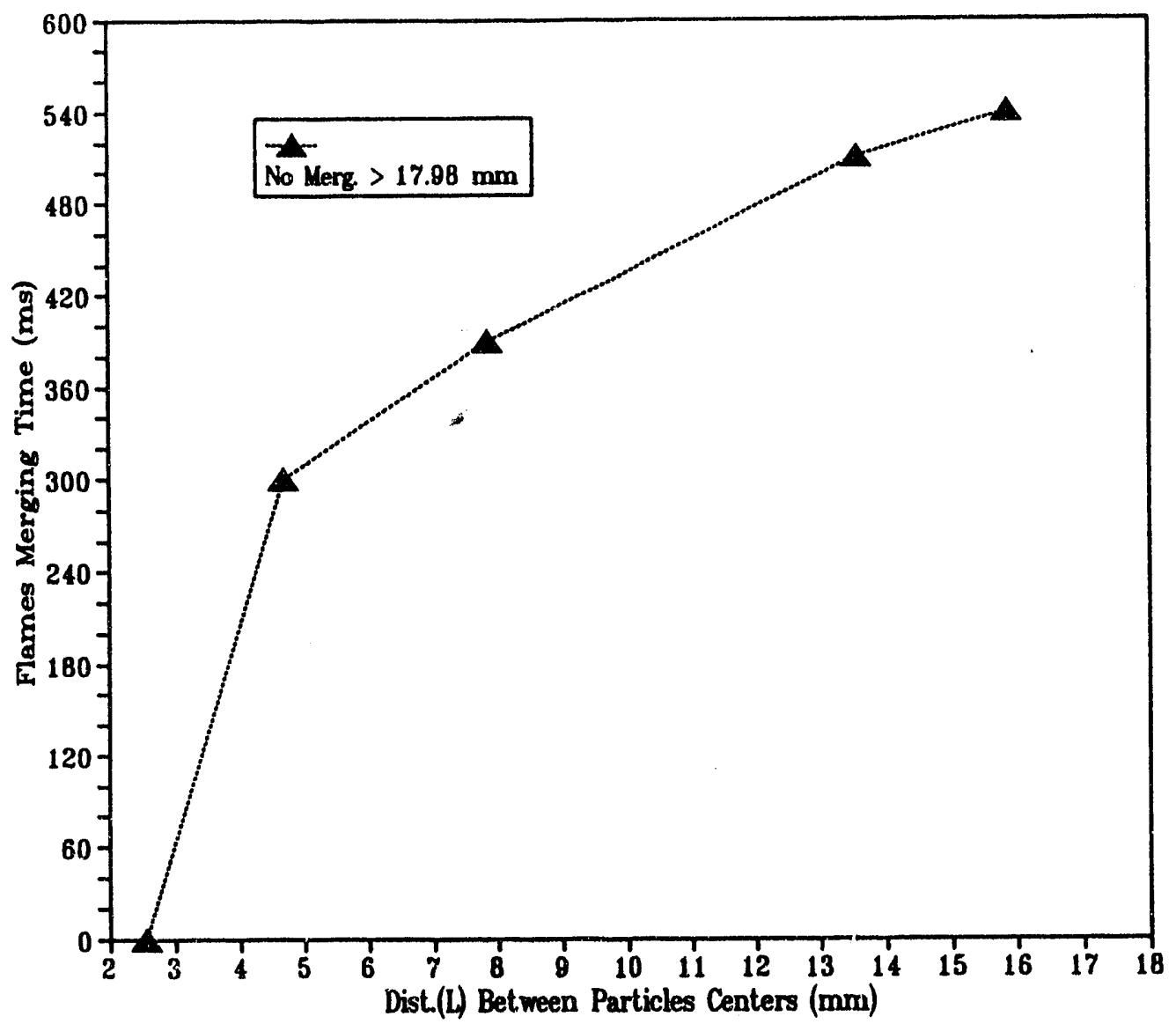

Figure 66. Variation of Flames Merging Time with Interparticle Spacing for Medium volatile Coal Two-Particle Arrays 


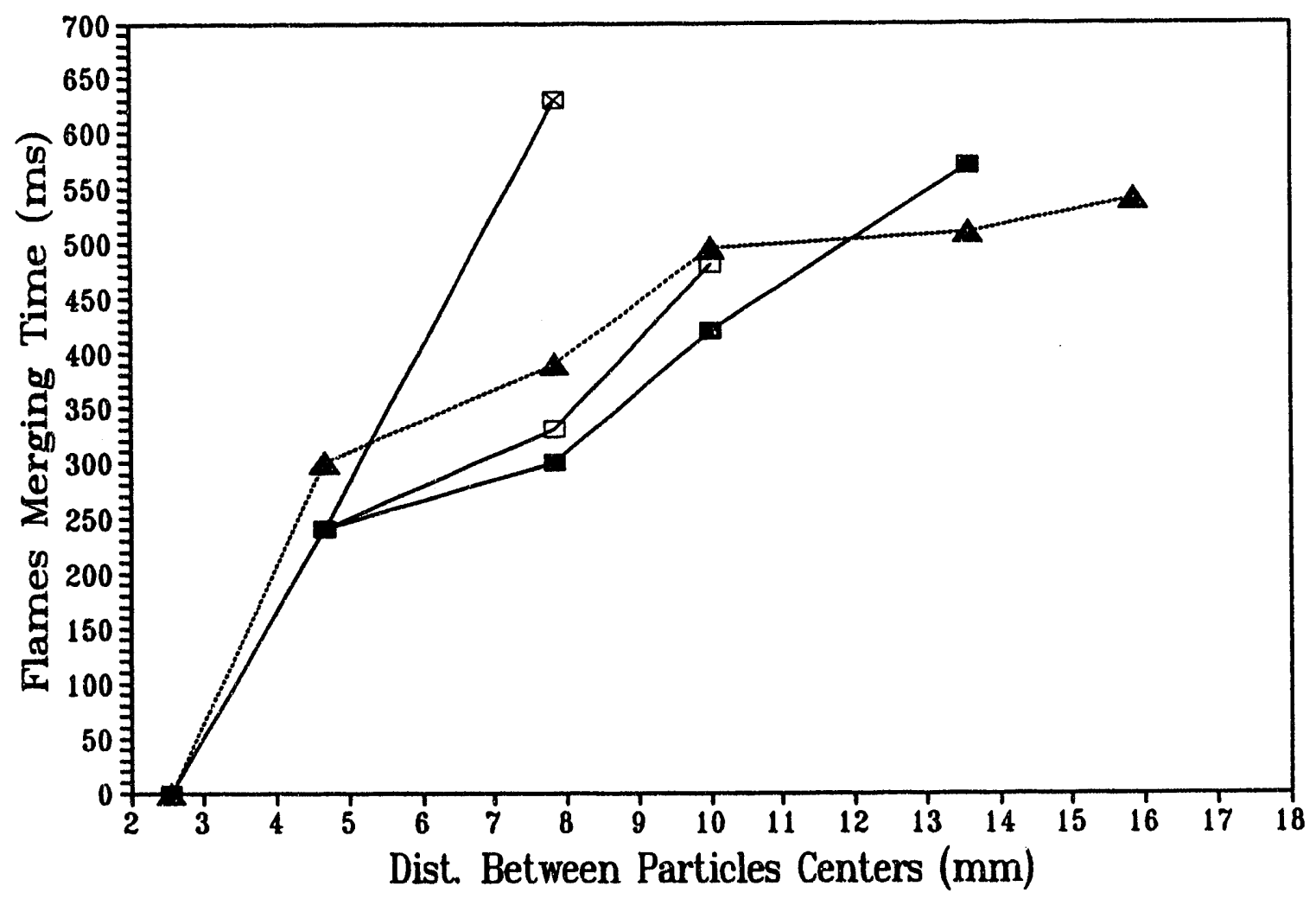

Figure 67. Comparison of Flames Merging Time variation with Interparticie Spacing for High and Medium Volatile Coal Two-Particle Arrays 
Table 11. Combustion Characteristics of Medium Volatile Coal Two-Particle Arrays

\begin{tabular}{|c|c|c|c|c|}
\hline 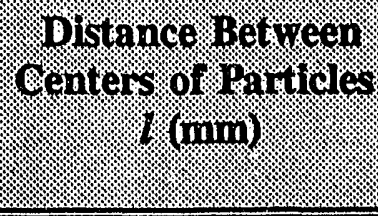 & $\begin{array}{l}\text { Ignition } \\
\text { Timés } \\
\text { (mis) }\end{array}$ & 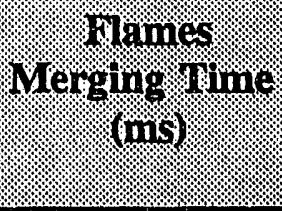 & \multicolumn{2}{|c|}{ Diameter } \\
\hline 2.54 & 30 & Immediately & 1.0 & 1.04 \\
\hline 4.67 & 300 & 300 & 1.01 & 1.04 \\
\hline 7.85 & 60 & 390 & 1.00 & 1.05 \\
\hline 13.58 & 30 & 510 & 0.98 & 1.0 \\
\hline 15.87 & 120 & 540 & 1.01 & 1.04 \\
\hline 17.98 & 60 & No Merging & 1.0 & 1.02 \\
\hline
\end{tabular}




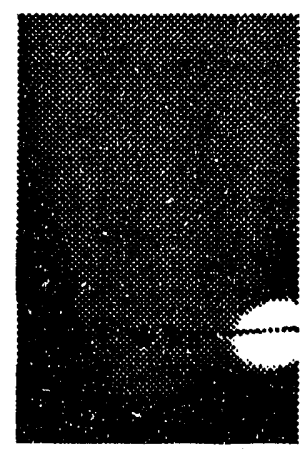

30

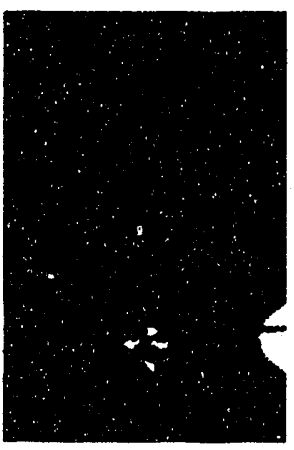

150

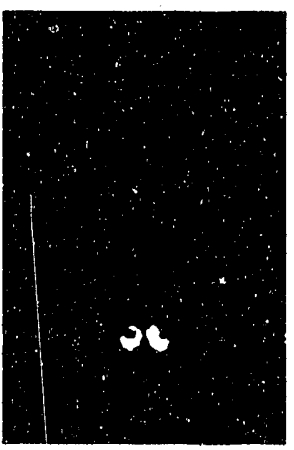

330

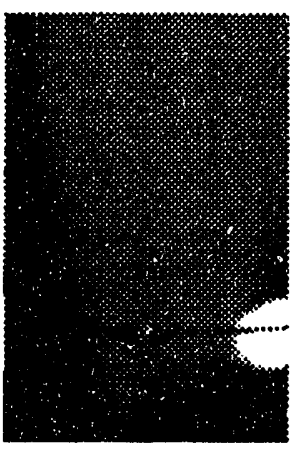

60

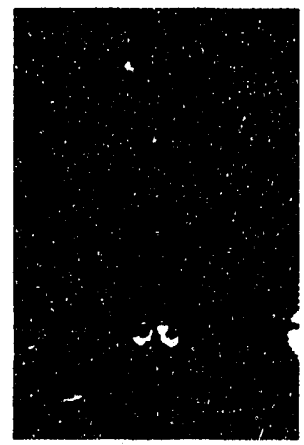

210

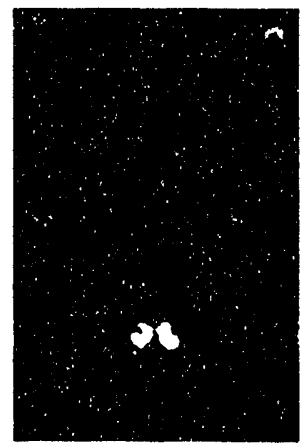

390

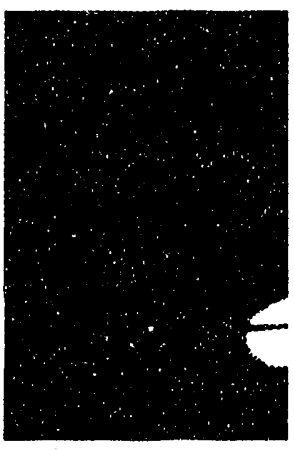

90

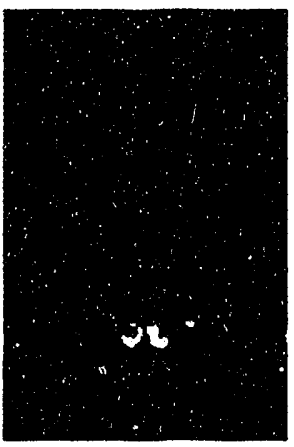

270

Figure 68a. Ignition and Combustion Sequence for Char Two-Particle

$$
\text { Array }(l=2.20 \mathrm{~mm})
$$




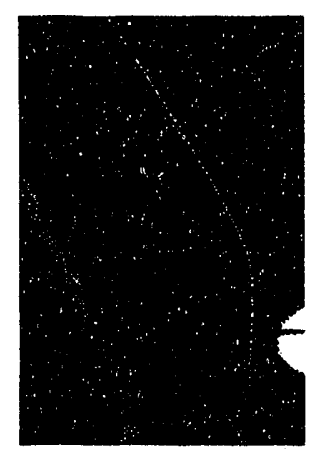

90

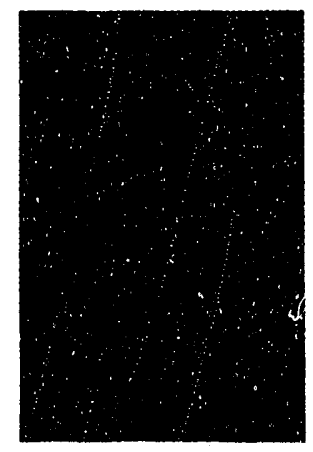

270

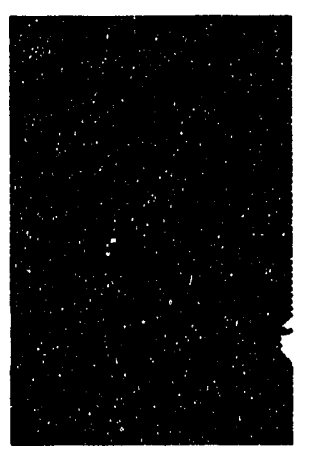

150

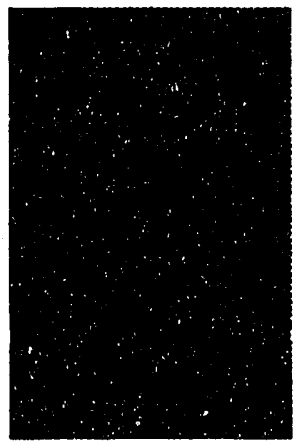

330

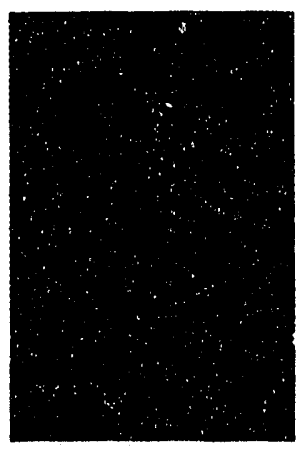

210

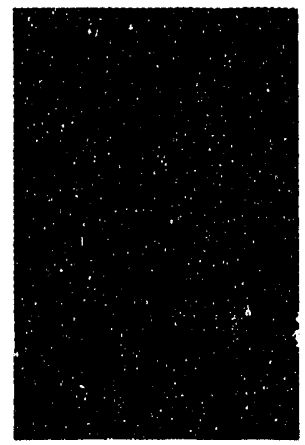

390

Figure 68b. Images of Ignition and Combustion Sequence with Boundary Detection Function for Char Two-Particle Array 


\section{CHAPTER VII}

\section{CONCLUSIONS}

Digital image processing techniques have been employed to investigate the ignition and combustion characteristics of isolated char/coal particles as well as twoparticle char/coal arrays. Preliminary experiments have been conducted on isolated candles and two-candle arrays in order to determine the burning rate and the effect of interactive combustion on the burning rate of the arrays.

The primary results are as follows:

i.) The isolated candle mass decreases as time increases. As the candle starts to burn, a flame is formed around its wick including a black inner zone possibly due to the formation of fuel rich core region.

ii.) A group flame is observed for the two-candle arrays. Upon increasing the inter candle spacing, the burn time first decreases and then later increases.

iii.) A group flame is formed for the two-particle arrays at closer interparticle spacing. iv.) Intermittent ignition does not occur for the high volatile particles when the two particles are at farther distances, while it occurs for an isolated high volatile particle. This suggests that radiation interaction between the particles might be occurring.

v.) Char/coal particles in vitiated environment seem to ignite heterogeneously at the leading edge of the particle. 


\section{CHAPTER VIII}

\section{RECOMMENDATIONS}

The digital image processing system employed in this research project has been found to fulfill the intended objective. However, in order to achieve more conclusive results the following need to be considered:

i.) Improve the technique of exposing the particles to the hot environment provided by the flat flame burner, that is the shielding plate positioned on top of the burner needs to be cooled to prevent preheating of the particles.

ii.) Conduct further experiments for the two-particle high volatile coal arrays at farther interparticle distances while shielding the particles from each other, thus reducing the radiation interaction between them, if any. This should assist in building a fundamental understanding about the intermittent volatile ignition and combustion phenomena.

ii.) Conduct further experiments at zero oxygen concentration to achieve conclusive results about the initial ignition mode, heterogeneous or homogeneous. Under zero oxygen concentration, coal particles should pyrolyze releasing volatile (gray streaks) without burning, thus gray streaks will be observed. On the other hand, if heterogeneous ignition initiates first, then gray streaks will not be observed during pyrolysis.

iii.) Upgrade the digital image processing system by installing the interface control module (ICM) to the camera which serves to enable the camera entire functions for optimum system efficiency. 


\section{REFERENCES}

Annamalai, K. and Caton, J., 1987, "Distinctive Burning Characteristics of Carbon Particles," The Canadian Journal of Chemical Engineering, Vol. 65, pp. 10271032.

Annamalai, K. and Durbetaki, P., 1977, "A Theory on Transition of Ignition Phase of Coal Particles," Comb. and Fl., Vol. 29, pp. 193-208.

Annamalai, K. and Ryan, W., 1992, "Interactive Transport Processes in Gasification and Combustion, Part I: Liquid Drop Arrays and Clouds," Prog. Energy and Comb. Sc., Vol. 18, pp. 221-295.

Annual Energy Review, 1991, Energy Information Administration, Office of Energy Markets and End Use, U.S. Department of Energy, Washington DC.

Bandyopadhyay, S. and Bhaduri, D., 1972, "Predictions of Ignition Temperatures of a Single Coal Particle," Comb. and Fl., Vol. 18, pp. 411-415.

Gieras, M., Klemens, R., Wolanski, P., and Wojcicki, S., 1986, "Experimental and Theoretical Investigation into the Ignition and Combustion Processes of Single Coal Particles under Zero and Normal Gravity Conditions," 21st Symp. (Int.) on Comb., The Combustion Institute, Pittsburgh, PA, pp. 315-323.

Howard, J. B. and Essenhigh, R. H., 1966, "Pyrolysis of Coal Particles in Pulverized Fuel Flames," Comb. and Fl., Vol. 10, pp. 92-93.

Juniper, L. A. and Wall, T. F., 1980, "Combustion of Particles in a Large Pulverized Brown Coal Flame," Comb. and Fl., Vol. 39, pp. 69-81.

Karcz, A. and Zembrzuski, M., 1982, "Investigation of Coal Combustion Using a Thermographic Method," Comb. and Fl., Vol. 45, pp. 95-98.

Karcz, H., Kordylewski, W. and Rybak, W., 1980, "Evaluation of Kinetic Parameters of Coal Ignition," Fuel, Vol. 59, pp. 799-802.

Kobayashi, H., 1972, "Rapid Decomposition Mechanisms of Pulverized Coals," Ph.D. Thesis, Chemical Engineering, MIT, Cambridge Mass. 
Makel, D. B. and Kennedy, I. M., 1991, "Imaging of Mixing and Soot in a Fuel Rich Combustor, ${ }^{n}$ Western States Section of the Combustion Institute, No. 91-35, Boulder, Colorado.

McLean, W. J., Hardesty, D. R., and Poul, J. H., 1981, "Direct Observations of Devolatilizing Pulverized Coal Particles in a Combustion Environment," 18th Symp. (Int.) on Comb., The Combustion Institute, Pittsburgh, PA, pp. 12391248.

Murigal, M. G. and Hollingsworth, D. K., 1989, "Organized Motion in a Very High Reynolds Number Jet," Phys. and Fluids, Vol. 1, pp. 1615-1622.

Ryan, W. and Annamalai, K., 1991, "Group Ignition of a Cloud of Coal Particles," Journal of Heat Transfer, Vol. 113, pp. 677-687.

Seeker, W. R., Samuelsen, G. S., Heap, M. P. and Trolinger, J. D., 1981, "The Thermal Decomposition of Pulverized Coal Particles," 18th Symp. (Int.) on Comb., The Combustion Institute, Pittsburgh, PA, pp. 1213-1226.

Solomon, P. R., Chien, P. L., Carangelo, R. M., Serio, A. M. and Markham, J. R., 1990, "New Ignition Phenomenon in Coal Combustion," Comb. and Fl., Vol. 79, pp. 214-2,15.

Spalding, D. B., M. A., 1951, "Combustion of Fuel Particles," Fuel, Vol. 30-6, pp. 121-130.

Street, P. J., Weight, R. P. and Lightman, P., 1969, "Further Investigations of Structural Changes Occurring in Pulverized Coal Particles During Rapid Heating," Fuel, Vol. 48., pp. 343-365.

Timothy, L. D., Sarofim, A. F. and Beer, J. M., 1982, "Characteristics of Single Particle Coal Combustion," 19th Symp. (Int.) on Comb., The Combustion Institute, Pittsburgh, PA, pp. 1123-1130.

Tomeczek, J. and Wojcik, J., 1990, "A Method of Direct Measurement of Solid Fuel Particle Ignition Temperature," 23rd Symp. (Int.) on Comb., The Combustion Institute, Pittsburgh, PA, pp. 1107-1127.

Tu, C. M., Davis, H. and Hottel, H. C., 1934, "Combustion Rate of Carbon: Combustion of Spheres in Flowing Gas Streams, "Ind. Engg. Chem., Vol. 26, pp. 749-757. 


\section{Supplementary Sources Consulted}

Bartok, W., and Sarofim, A., 1991, Fossil Fuel Combustion, John Wiley \& Sons, New York.

Baxes, G. A., 1984, Digital Image Processing: a Practical Primer, Prentice-Hall, New Jersey.

Castleman, K. R., 1979, Digital Image Processing, Prentice-Hall, New Jersey.

Gonzalez, R. C., 1987, Digital Image Processing, Addison-Wesley, Mass.

Green, W. B., 1983, Digital Image Processing: a Systems Approach, Van Nostrand Reinhold, New York.

Kanury, A. M., 1975, Introduction to Combustion Phenomena, Gordon and Breach, New York.

Kuo, K., 1986, Principles of Combustion, John Wiley \& Sons, New York.

Pratt, W. K., 1987, Digital Image Processing, John Wiley \& Sons, New York.

Rabbani, M., 1991, Digital Image Compression Techniques, Spie Optical Engineering Press, WA.

Schalkoff, R. J., 1989, Digital Image Processing and Computer Vision, John Wiley \& Sons, New York. 


\section{APPENDIX A \\ LENS SELECTION CRITERIA}

The CID2250D camera, with the appropriate lens, is applicable to almost any task required of a video camera. The lens gears the camera to specific tasks providing tremendous latitude in system application. The selection of lenses involves a consideration of a number of variables. This appendix will present a criteria for selecting the right lens applicable to ones system for optimum efficiency.

i.) Focal length ( $f$ ): the distance between the prime focus point (center of the sensor) and the center of the lens is called the focal length. Because lenses are available in fixed local lengths (except zoom lenses), it is often best to pick a lens focal lengih based on the approximate lens-to-object distance, then vary the distance for the exact match desired. Lenses range in focal length from 9 to $75 \mathrm{~mm}$ for the CID2250 camera. Generally this formula applies in determining the focal length (f):

$f=(8.68 \times D) /(A+0.34) \mathrm{mm}$, or

$1 / f=1 / l+1 / D$

where D (in inches) is the object distance (the distance between the object and the lens), A (in inches) is the object height, and $l$ is the lens-to-array distance in $\mathrm{mm}$.

ii.) Extension tube length (XL): Since there is a limit to the minimum lens-to-object distance for lenses, extension tubes are used on these lenses, allowing the lens-to-array distance to be increased, thus further reducing the minimum object distance. The 
following formula enables the appropriate selection of an extension tube:

$D=(l \times f) /(25.4 \times X L)$ inches

where $\mathrm{XL}$ is the extension tube length in $\mathrm{mm}$.

iii.) Magnification: When enlarging the size of the object is desired, a magnification lens

is the answer. The following formula shows the relationship with other variables:

$\mathrm{D} / \mathrm{f}=(\mathrm{M}+1) / \mathrm{M}$

where $M$ is the magnification desired. Also, $M$ is related to $D$ and $I$ as follows:

$l / \mathrm{D}=\mathrm{M}$

Figure 69 shows the relationship of focal length versus magnification at an olject distance of 120 and $250 \mathrm{~mm}$. 


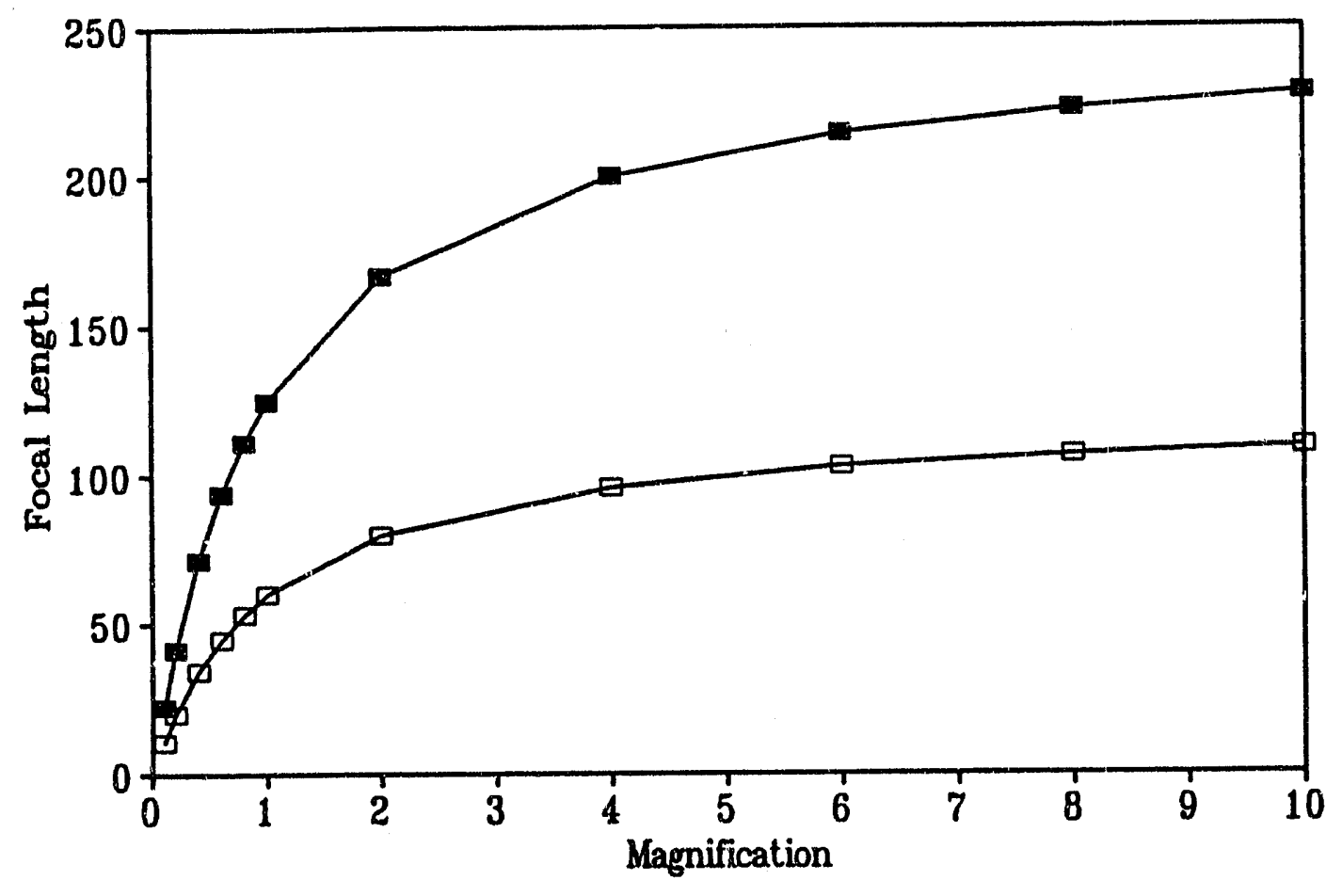

$\rightarrow-$ Obj Dist $=250 \mathrm{~mm}-120 \mathrm{~mm}$

Figure 69. Variation of Focal Length with Magnification 


\section{APPENDIX B}

\section{EXPERIMENTAL DATA}

This appendix includes some of the experimental data obtained. Data is presented in tabulated form as well as in graphical illustration. Tables 12 through 17 summarize the experimental data obtained for candles. Figure $\mathbf{7 0}$ shows the variation of candles mass calculated from regression analysis with time. 
Table. 12 Variation of Mass with Time for Two-Candle Array $(l=6 \mathrm{~mm})$

\begin{tabular}{|c|c|c|c|c|}
\hline Time (8) & Total Yass (R) & Mass Per Candle (R) & Mass/Mass Initial & Mass-Rerression \\
\hline 0 & 2.25 & 1.13 & 1.00 & 1.12 \\
\hline 15 & 2.19 & 1.10 & 0.87 & 1.10 \\
\hline 30 & 2.17 & 1.09 & 0.96 & 1.08 \\
\hline 45 & 2.13 & 1.07 & 0.95 & 1.06 \\
\hline 60 & 2.07 & 1.04 & 0.92 & 1.04 \\
\hline 75 & 2.06 & 1.03 & 0.92 & 1.02 \\
\hline 90 & 1.99 & 1.00 & 0.88 & 1.00 \\
\hline 105 & 1.87 & 0.89 & 0.88 & 0.98 \\
\hline 120 & 1.8 & 0.95 & 0.84 & 0.86 \\
\hline 135 & 1.87 & 0.84 & 0.83 & 0.94 \\
\hline 150 & 1.85 & 0.93 & 0.82 & 0.02 \\
\hline 165 & 1.82 & 0.91 & 0.81 & 0.90 \\
\hline 180 & 1.74 & 0.87 & 0.77 & 0.88 \\
\hline 195 & 1.7 & 0.85 & 0.76 & 0.86 \\
\hline 210 & 1.68 & 0.84 & 0.75 & 0.84 \\
\hline 225 & 1.64 & 0.82 & 0.73 & 0.82 \\
\hline 240 & 1.59 & 0.80 & 0.71 & 0.79 \\
\hline 255 & 1.56 & 0.78 & 0.68 & 0.77 \\
\hline 270 & 1.5 & 0.75 & 0.67 & 0.75 \\
\hline 285 & 1.45 & 0.73 & 0.64 & 0.73 \\
\hline 300 & 1.43 & 0.72 & 0.64 & 0.71 \\
\hline 315 & 1.37 & 0.68 & 0.61 & 0.69 \\
\hline 330 & 1.35 & 0.68 & 0.60 & 0.67 \\
\hline
\end{tabular}

\begin{tabular}{|lr|}
\hline \multicolumn{2}{|c|}{ Regression Output } \\
\hline Two Candles $(6 \mathrm{~mm})$ \\
\hline Constant & 1.1235869565217 \\
Std Brr of Y Est & 0.0064454887510551 \\
R Squared & 0.99796538223114 \\
Na. of Observations & 23 \\
Degrees of Freedom & 21 \\
& \\
X Coefficient(s) & \\
Std Err of Coef. & -0.001370882740448 \\
\hline
\end{tabular}


Table 13. Variation of Mass with Time for Two-Candle Array $(l=9 \mathrm{~mm})$

\begin{tabular}{|c|c|c|c|c|}
\hline Time (s) & Total Mass (g) & Mass Per Candle (g) & Mass/Lnitial Mass & Mass-Regression \\
\hline 0 & 2.12 & 1.06 & 1 & 1.08 \\
\hline 15 & 2.1 & 1.05 & 0.99 & 1.06 \\
\hline 30 & 2.07 & 1.04 & 0.98 & 1.04 \\
\hline 45 & 2.02 & 1.01 & 0.95 & 1.02 \\
\hline 60 & 2.02 & 1.01 & 0.95 & 1.00 \\
\hline 75 & 1.98 & 0.99 & 0.93 & 0.88 \\
\hline 80 & 1.92 & 0.86 & 0.91 & 0.96 \\
\hline 105 & 1.88 & 0.94 & 0.89 & 0.94 \\
\hline 120 & 1.85 & 0.93 & 0.87 & 0.82 \\
\hline 135 & 1.78 & 0.89 & 0.84 & 0.80 \\
\hline 150 & 1.76 & 0.88 & 0.83 & 0.86 \\
\hline 165 & 1.74 & 0.87 & 0.82 & 0.86 \\
\hline 180 & 1.71 & 0.86 & 0.81 & 0.84 \\
\hline 185 & 1.64 & 0.82 & 0.77 & 0.82 \\
\hline 210 & 1.61 & 0.81 & 0.76 & 0.80 \\
\hline 225 & 1.55 & 0.78 & 0.73 & 0.78 \\
\hline 240 & 1.53 & 0.77 & 0.72 & 0.76 \\
\hline 255 & 1.5 & 0.75 & 0.71 & 0.74 \\
\hline 270 & 1.44 & 0.72 & 0.68 & 0.72 \\
\hline 285 & 1.42 & 0.71 & 0.67 & 0.70 \\
\hline 300 & 1.34 & 0.67 & 0.63 & 0.68 \\
\hline 315 & 1.31 & 0.66 & 0.62 & 0.66 \\
\hline 330 & 1.28 & 0.64 & 0.60 & 0.64 \\
\hline 331 & 1.27 & 0.64 & 0.60 & 0.64 \\
\hline & & & & \\
\hline
\end{tabular}

\begin{tabular}{|lr|}
\hline \multicolumn{2}{|c|}{ Two Candles (9mm apart) } \\
Regression Output: \\
\hline Constant & 1.0768709593106 \\
Std Err of Y Bst & 0.0085311608450357 \\
R Squared & 0.99636825573355 \\
Na. of Observations & 24 \\
Degrees of Preedom & 22 \\
& \\
$X$ Coefricient(s) & -0.001314809264046 \\
Std Er of Coef. & $1.69238475114 \mathrm{E}-05$ \\
\hline
\end{tabular}


Table 14. Variation of Mass with Time for Two-Candle Array $(l=12 \mathrm{~mm})$

\begin{tabular}{|c|c|c|c|c|}
\hline Time (s) & Total Mass (g) & Mass Per Candle (g) & Mass/Lnitial Mass & Mass-Regression \\
\hline 0 & 2.07 & 1.04 & 1.00 & 1.03 \\
\hline 15 & 2.03 & 1.02 & 0.98 & 1.01 \\
\hline 30 & 1.97 & 0.99 & 0.95 & 0.99 \\
\hline 45 & 1.89 & 0.05 & 0.81 & 0.97 \\
\hline 60 & 1.89 & 0.85 & 0.81 & 0.94 \\
\hline 75 & 1.85 & 0.93 & 0.89 & 0.92 \\
\hline 90 & 1.79 & 0.90 & 0.86 & 0.90 \\
\hline 105 & 1.77 & 0.89 & 0.86 & 0.08 \\
\hline 120 & 1.71 & 0.86 & 0.83 & 0.85 \\
\hline 135 & 1.65 & 0.83 & 0.80 & 0.83 \\
\hline 150 & 1.61 & 0.81 & 0.78 & 0.81 \\
\hline 165 & 1.59 & 0.80 & 0.77 & 0.78 \\
\hline 180 & 1.53 & 0.77 & 0.74 & 0.76 \\
\hline 195 & 1.51 & 0.76 & 0.73 & 0.74 \\
\hline 210 & 1.47 & 0.74 & 0.71 & 0.72 \\
\hline 225 & 1.98 & 0.69 & 0.67 & 0.69 \\
\hline 240 & 1.32 & 0.66 & 0.64 & 0.67 \\
\hline 255 & 1.3 & 0.65 & 0.63 & 0.65 \\
\hline 265 & 1.24 & 0.62 & 0.60 & 0.63 \\
\hline
\end{tabular}

\begin{tabular}{|c|c|c|}
\hline \multicolumn{3}{|c|}{$\begin{array}{l}\text { Two Candles (12mm apart) } \\
\text { Regression Output: }\end{array}$} \\
\hline $\begin{array}{l}\text { Constant } \\
\text { Std Er of Y Est } \\
\text { R Squared } \\
\text { Na of Observations } \\
\text { Degrees of Ireedom }\end{array}$ & & $\begin{array}{r}1.0336490016794 \\
0.0098497892040932 \\
0.99430020076776 \\
19 \\
17\end{array}$ \\
\hline $\begin{array}{l}X \text { Coeficient(s) } \\
\text { Std EIr of Coef. }\end{array}$ & $\begin{array}{l}-0.001505598059339 \\
2.764748310251 \mathrm{E}-05\end{array}$ & \\
\hline
\end{tabular}


Table 15. Variation of Mass with Time for Two-Candle Array $(l=15 \mathrm{~mm})$

\begin{tabular}{|c|c|c|c|c|}
\hline Time (s) & Total Mass (g) & Mass Per Candle (g) & Mass/Initial Mass & Mass-Regressicin \\
\hline 0 & 2.19 & 1.10 & 1.00 & 1.09 \\
\hline 15 & 2.16 & 1.08 & 0.99 & 1.07 \\
\hline 30 & 2.13 & 1.07 & 0.87 & 1.05 \\
\hline 45 & 2.06 & 1.03 & 0.94 & 1.03 \\
\hline 60 & 2.04 & 1.02 & 0.93 & 1.01 \\
\hline 75 & 1.99 & 1.00 & 0.91 & 0.99 \\
\hline 90 & 1.97 & 0.99 & 0.90 & 0.88 \\
\hline 105 & 1.90 & 0.95 & 0.87 & 0.96 \\
\hline 120 & 1.86 & 0.93 & 0.85 & 0.94 \\
\hline 135 & 1.81 & 0.91 & 0.83 & 0.02 \\
\hline 150 & 1.77 & 0.89 & 0.81 & 0.80 \\
\hline 165 & 1.74 & 0.87 & 0.79 & 0.88 \\
\hline 180 & 1.72 & 0.86 & 0.79 & 0.86 \\
\hline 195 & 1.69 & 0.85 & 0.77 & 0.84 \\
\hline 210 & 1.64 & 0.82 & 0.75 & 0.82 \\
\hline 225 & 1.59 & 0.80 & 0.73 & 0.80 \\
\hline 240 & 1.43 & 0.72 & 0.65 & 0.78 \\
\hline 255 & 1.52 & 0.76 & 0.69 & 0.76 \\
\hline 270 & 1.50 & 0.75 & 0.68 & 0.74 \\
\hline 285 & 1.47 & 0.74 & 0.67 & 0.72 \\
\hline 300 & 1.42 & 0.71 & 0.65 & 0.70 \\
\hline 315 & 1.38 & 0.69 & 0.63 & 0.68 \\
\hline 330 & 1.33 & 0.67 & 0.61 & 0.66 \\
\hline 333 & 1.31 & 0.66 & 0.60 & 0.66 \\
\hline
\end{tabular}

\begin{tabular}{|lr|}
\hline \multicolumn{2}{|c|}{ Two Candles (15mm apart) } \\
Regression Output: \\
\hline Constant & 1.092984066607 \\
Std Err of Y Est & 0.015618130579848 \\
R Squared & 0.98793520159219 \\
Na of Observations & 24 \\
Degrees of Preedom & 22 \\
& \\
X Coefficient(s) & \\
Std Err of Coef & -0.001313376356242 \\
\hline
\end{tabular}


Table 16. Variation of Mass with Time for Two-Candle Array $(l=17 \mathrm{~mm})$

\begin{tabular}{|c|c|c|c|c|}
\hline Time (s) & Total Mass (g) & Mass Per Candle (g) & Mass/Initial Mass & Mass-Regression \\
\hline 0 & 2.19 & 1.10 & 1.00 & 1.10 \\
\hline 15 & 2.16 & 1.08 & 0.99 & 1.08 \\
\hline 30 & 2.14 & 1.07 & 0.98 & 1.06 \\
\hline 45 & 2.09 & 1.05 & 0.85 & 1.04 \\
\hline 60 & 2.04 & 1.02 & 0.93 & 1.02 \\
\hline 75 & 1.89 & 1.00 & 0.91 & 1.00 \\
\hline 80 & 1.96 & 0.98 & 0.89 & 0.88 \\
\hline 105 & 1.92 & 0.96 & 0.88 & 0.96 \\
\hline 120 & 1.89 & 0.96 & 0.86 & 0.94 \\
\hline 135 & 1.85 & 0.83 & 0.84 & 0.82 \\
\hline 150 & 1.82 & 0.81 & 0.83 & 0.90 \\
\hline 165 & 1.78 & 0.89 & 0.81 & 0.88 \\
\hline 180 & 1.68 & 0.84 & 0.77 & 0.86 \\
\hline 195 & 1.69 & 0.85 & 0.77 & 0.84 \\
\hline 210 & 1.86 & 0.83 & 0.76 & 0.82 \\
\hline 225 & 1.62 & 0.81 & 0.74 & 0.80 \\
\hline 240 & 1.58 & 0.78 & 0.72 & 0.78 \\
\hline 255 & 1.54 & 0.77 & 0.70 & 0.76 \\
\hline 270 & 1.46 & 0.73 & 0.67 & 0.74 \\
\hline 285 & 1.45 & 0.73 & 0.66 & 0.72 \\
\hline 300 & 1.38 & 0.89 & 0.63 & 0.70 \\
\hline 315 & 1.38 & 0.69 & 0.63 & 0.68 \\
\hline 330 & 1.35 & 0.68 & 0.62 & 0.66 \\
\hline 338 & 1.31 & 0.66 & 0.60 & 0.65 \\
\hline & & & & \\
\hline
\end{tabular}

\begin{tabular}{|lr|}
\hline \multicolumn{2}{|c|}{$\begin{array}{c}\text { Two Candles (17mm apart) } \\
\text { Regression Output: }\end{array}$} \\
\hline Constant & 1.1004929640297 \\
Std Err of Y Est & 0.0083458997524078 \\
R Squared & 0.99657048618294 \\
Na. of Observations & 24 \\
Degrees of Freedom & 22 \\
& \\
X Coefficient(s) & \\
Std Err of Coef. & -0.001317888007915 \\
\hline
\end{tabular}


Table 17. Variation of $40 \%$ Burn Time with Inter-Candle Spacing for Two Candle Arrays

Candle's Diameter $(d)=1.12 \mathrm{~mm}$

Candle's Radius $(\mathrm{a})=0.56 \mathrm{~mm}$

Distance Between Candles Varied From $6 \mathrm{mrn}$ to $17 \mathrm{~mm}$

Interparticle Distance (l/a) Varied From $10.71 \mathrm{~mm}$ to 30.36

Candles

Two Candles in Contact (6mm)

Tro Candles ( $9 \mathrm{~mm}$ apart)

Two Candles (12mm apart)

Two Candles (15 mm apart)

Two Candles (17 mm apart)
Time to Burn 40\% Mass Interparticle Dist. (1/a)

330

331

265

333

338
10.71

16.07

21.43

26.79

30.36 


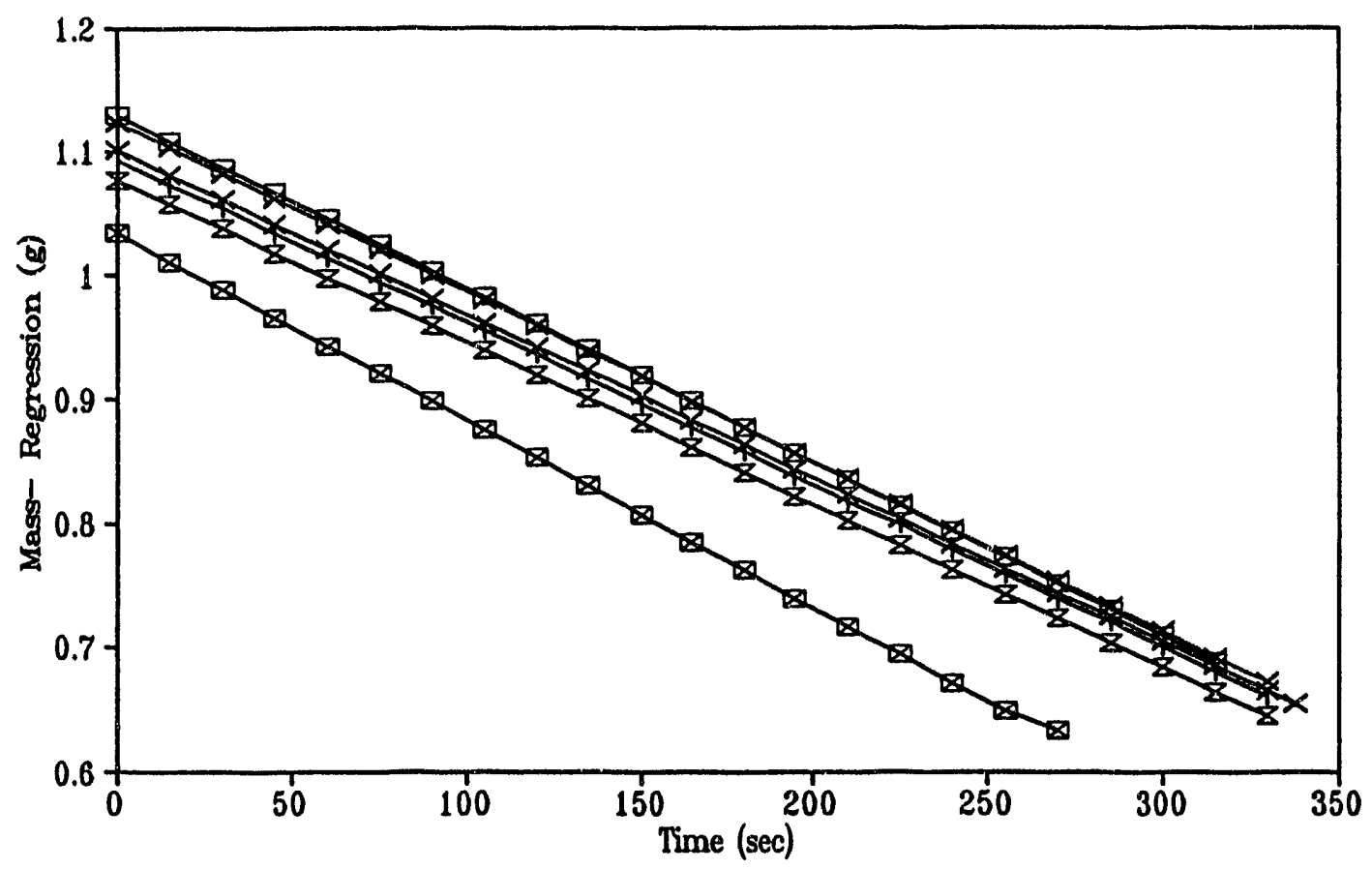

- - Single Candle $\rightarrow$ Tro Candles $(6 \mathrm{~mm})-z$ Two Candles $(9 \mathrm{~mm})$
- Tro Candles $(12 \mathrm{~mm}) \rightarrow$ Tro Candies $(15 \mathrm{~mm}) \rightarrow$ Two Candles $(17 \mathrm{~mm})$

Figure 70. Variation of Mass (Regression) with Time for Candles 

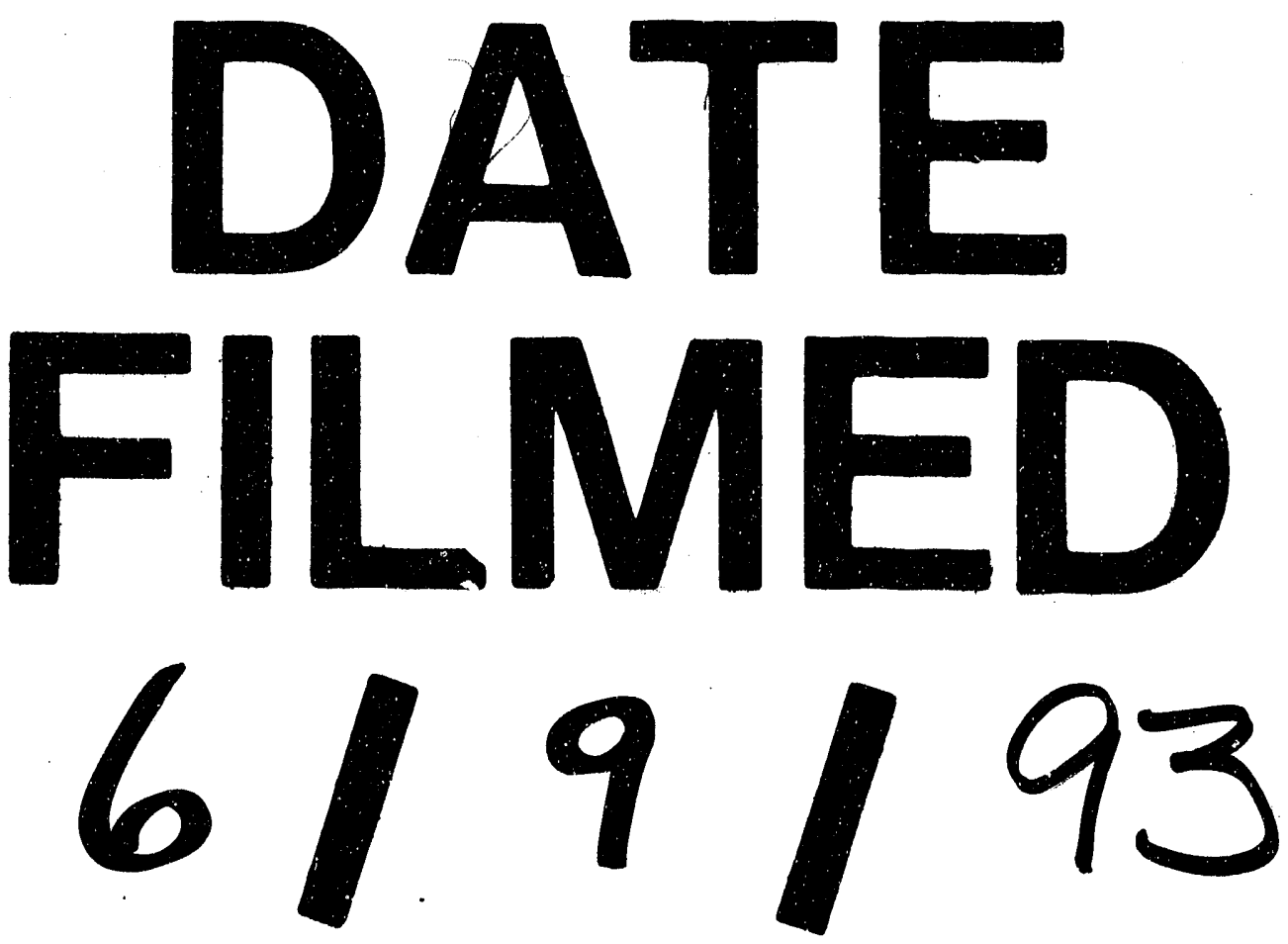
